\title{
The glycation pathway in type 2 diabetes and cardiovascular disease
}

Citation for published version (APA):

Engelen, L. (2012). The glycation pathway in type 2 diabetes and cardiovascular disease. [Doctoral Thesis, Maastricht University]. Ipskamp Drukkers B.V., Enschede. https://doi.org/10.26481/dis.20121012le

Document status and date:

Published: 01/01/2012

DOI:

10.26481/dis.20121012le

Document Version:

Publisher's PDF, also known as Version of record

\section{Please check the document version of this publication:}

- A submitted manuscript is the version of the article upon submission and before peer-review. There can be important differences between the submitted version and the official published version of record.

People interested in the research are advised to contact the author for the final version of the publication, or visit the DOI to the publisher's website.

- The final author version and the galley proof are versions of the publication after peer review.

- The final published version features the final layout of the paper including the volume, issue and page numbers.

Link to publication

\footnotetext{
General rights rights.

- You may freely distribute the URL identifying the publication in the public portal. please follow below link for the End User Agreement:

www.umlib.nl/taverne-license

Take down policy

If you believe that this document breaches copyright please contact us at:

repository@maastrichtuniversity.nl

providing details and we will investigate your claim.
}

Copyright and moral rights for the publications made accessible in the public portal are retained by the authors and/or other copyright owners and it is a condition of accessing publications that users recognise and abide by the legal requirements associated with these

- Users may download and print one copy of any publication from the public portal for the purpose of private study or research.

- You may not further distribute the material or use it for any profit-making activity or commercial gain

If the publication is distributed under the terms of Article $25 \mathrm{fa}$ of the Dutch Copyright Act, indicated by the "Taverne" license above, 
THE GLYCATION PATHWAY

IN TYPE 2 DIABETES AND CARDIOVASCULAR DISEASE 
(C) Lian Engelen, Maastricht 2012

Cover picture: Imperfect reflection by Rolf Lorenz

Cover design: Jean Scheijen | www.vierdrie.nl

Production: Ipskamp Drukkers B.V., Enschede

ISBN: 978-94-6191-421-7 


\title{
THE GLYCATION PATHWAY \\ IN TYPE 2 DIABETES AND CARDIOVASCULAR DISEASE
}

\author{
PROEFSCHRIFT \\ ter verkrijging van de graad van doctor aan de Universiteit Maastricht, \\ op gezag van de Rector Magnificus, Prof. dr. L.L.G. Soete, \\ volgens het besluit van het College van Decanen, \\ in het openbaar te verdedigen \\ op vrijdag 12 oktober 2012 om 12.00 uur
}

door

Lian Engelen 


\section{Promotores}

Prof. dr. C.D.A. Stehouwer

Prof. dr. C.G. Schalkwijk

\section{Copromotor}

Dr. I. Ferreira

\section{Beoordelingscommissie}

Prof. dr. H.A.J. Struijker-Boudier (voorzitter)

Prof. dr. L.M.A. van Bortel

Prof. dr. N.C. Schaper

Prof. dr. Y.T. van der Schouw, UMC Utrecht

Dr. M.T. Schram

Dr. A.J. Smit, UMC Groningen

Financial support by the Netherlands Heart Foundation and the Dutch Diabetes Research Foundation for the publication of this thesis is gratefully acknowledged.

Financial support from ESAOTE is also gratefully acknowledged. 


\section{CONTENTS}

$\begin{array}{lll}\text { Chapter } 1 & \text { General introduction } & 7\end{array}$

Part 1: Interventions in the glycation pathway

Chapter 2 Current interventions in the glycation pathway: evidence from clinical studies

Chapter 3 Improved glycaemic control induced by both metformin and 65 repaglinide is associated with a reduction in blood levels of 3 deoxyglucosone in non-obese patients with type 2 diabetes

Chapter 4 Irbesartan treatment does not influence plasma levels of the advanced glycation endproducts $\mathrm{N}^{\varepsilon}$ (1-carboxymethyl)lysine and $\mathrm{N}^{\varepsilon}$ (1-carboxyethyl)lysine in patients with type 2 diabetes and microalbuminuria. A randomized controlled trial

Part 2: The glycation pathway and cardiovascular disease

Chapter 5 Reference intervals for common carotid intima-media thickness measured with echotracking - relation with risk factors

Chapter 6 Polymorphisms in glyoxalase 1 gene are not associated with vascular complications: the Hoorn and CODAM studies

Chapter 7 The association between the -374T/A polymorphism of the receptor for advanced glycation endproducts gene and blood pressure and arterial stiffness is modified by glucose metabolism status: the Hoorn and CODAM studies

Chapter 8 Plasma levels of advanced glycation endproducts are not independently associated with cardiovascular disease in individuals with or without type 2 diabetes: the Hoorn and CODAM Studies

Chapter 9 General discussion 

CHAPTERP 1

\section{SEKERAL INTRODUCTION}




\section{GENERAL INTRODUCTION}

Diabetes is a common metabolic disease currently affecting around 346 million people worldwide, ${ }^{1,2}$ which is expected to rise to 552 million by $2030 .^{3}$ In the Netherlands, 740,000 individuals had diabetes in 2007 , which is expected to rise to 1,3 million in $2025 .{ }^{4}$ Micro- and macrovascular diseases cause most of the excess morbidity and mortality in diabetes. Specifically, adults with diabetes experience a 2-fold increased risk for cardiovascular events ${ }^{5}$ and cardiovascular death ${ }^{6}$ relative to those without diabetes. Large studies in type $1^{7}$ and type 2 diabetes ${ }^{8}$ have established that hyperglycaemia plays an important role in the pathogenesis of microvascular complications such as retinopathy, nephropathy and neuropathy, and macrovascular complications such as atherosclerotic disease. Various mechanisms have been proposed to explain the increased cardiovascular risk through hyperglycaemia including the increased formation of advanced glycation endproducts (AGEs) by non-enzymatic glycation of proteins - the glycation pathway. ${ }^{9-11}$

This thesis comprises a number of epidemiological studies that are aimed at investigating 1 ) possible interventions in the glycation pathway and 2) the role of the glycation pathway in the aetiology of (type 2 diabetes-related) cardiovascular disease. In addition, the establishment of reference intervals for one of the markers of cardiovascular disease used in this thesis, common carotid artery intima-media thickness, is included. Accordingly, first, some background will be provided regarding type 2 diabetes and its micro- and macrovascular complications, and the role of the glycation pathway therein.

\section{DIABETES}

Diabetes mellitus is a group of metabolic diseases characterized by elevated plasma glucose levels (i.e. hyperglycaemia) as a result of defects in insulin secretion and/or insulin action. $^{12}$ The majority of individuals with diabetes suffer from type 1 or type 2 diabetes.

Type 1 diabetes accounts for about 5-10\% of the prevalence of diabetes and results from a cellular-mediated autoimmune destruction of the beta cells of the pancreas, which commonly occurs in childhood or adolescence, but can occur at any age and often leads to absolute insulin deficiency. ${ }^{12}$

Type 2 diabetes comprises about $90 \%$ of all individuals with diabetes. ${ }^{1,3}$ Although type 2 diabetes results from peripheral insulin resistance, i.e., tissues have become less sensitive to the metabolic effects of insulin, insulin secretion is also defective in these patients and insufficient to compensate for the insulin resistance. ${ }^{12}$ The diagnosis of type 2 
diabetes is established whenever, on more than one occasion, fasting plasma glucose is $\geq 7.0 \mathrm{mmol} / \mathrm{L}$ and/or 2-hour postload plasma glucose (i.e. after $75 \mathrm{~g}$ orally administered glucose) $\geq 11.1 \mathrm{mmol} / \mathrm{L}^{13,14}$ Obesity and physical inactivity are regarded the most important risk factors for the development of type 2 diabetes and are thought to affect primarily insulin sensitivity, ${ }^{1,15}$ and recent genome-wide association studies have identified several susceptibility loci for type 2 diabetes that mostly affect beta cell function. ${ }^{16}$

The age-standardized global rise in diabetes prevalence from 1980 to 2008 was 8.3 to $9.8 \%$ and 7.5 to $9.2 \%$ in men and women, respectively, ${ }^{2}$ which has led to an estimated 193 million (153 million in 1980 to 346 million in 2008) additional cases of diabetes in this period. $^{2}$ About $70 \%$ of this global increase in the number of individuals living with diabetes can be attributed to population growth and ageing. However, rising obesity rates ${ }^{17}$ have fuelled the epidemic, probably accounting for much of the remaining $30 \%$ of the increase. $^{2,18}$

\section{MICRO- AND MACROVASCULAR COMPLICATIONS}

Long-term microvascular complications of diabetes include retinopathy with potential loss of vision; nephropathy leading to renal failure; and peripheral and autonomic neuropathy with risk of foot ulcers, amputations and gastrointestinal and genitourinary dysfunction. ${ }^{12}$ Due to improvements in the management of individuals with diabetes, the incidence of these microvascular complications has declined over the last decades. ${ }^{19}$ However, the agestandardized prevalence of any kind of diabetic retinopathy is $25 \%$ among individuals with type 2 diabetes, which increases with diabetes duration (to more than $50 \%$ when $>10$ years) $)^{20}$ and around $25 \%$ of the individuals who have type 2 diabetes for at least ten years suffers from microalbuminuria. ${ }^{21,22}$ Importantly, diabetic retinopathy is estimated to account for $5 \%$ of all cases of blindness worldwide ${ }^{23}$ and diabetic nephropathy is currently the leading cause of end-stage kidney disease. ${ }^{24}$ Two landmark studies, the Diabetes Control and Complications Trial (DCCT) ${ }^{7}$ and the United Kingdom Prospective Diabetes Study (UKPDS), ${ }^{8}$ showed that intensive control of hyperglycaemia can reduce the occurrence or progression of retinopathy, nephropathy and neuropathy in individuals with type 1 and type 2 diabetes, respectively. These results strongly suggest that hyperglycaemia is responsible for diabetic microvascular complications, ${ }^{25}$ although a recent meta-analysis on the effect of intensive glucose-lowering treatment in type 2 diabetes showed a significant reduction in incident microalbuminuria, without significant benefit on retinopathy or neuropathy. ${ }^{26}$ 
Individuals with diabetes also have an increased risk of developing macrovascular complications such as atherosclerotic cardiovascular, peripheral arterial and cerebrovascular disease. ${ }^{12}$ Large meta-analyses (including $>500,000$ individuals) have shown that, after accounting for other risk factors (i.e. age, smoking status, body-mass index, and systolic blood pressure), diabetes more than doubled the risk for coronary heart disease and ischaemic stroke ${ }^{5}$ and vascular death. ${ }^{6}$ Furthermore, in individuals with no history of diabetes, fasting glucose between 5.6 and $6.1 \mathrm{mmol} / \mathrm{L}$ and between 6.1 and $7.0 \mathrm{mmol} / \mathrm{L}$ already independently increased the risk of coronary heart disease by about 11 and $17 \%$, respectively. ${ }^{5}$ Whether the spectrum of dysglycaemia, including diabetes, is causally related to macrovascular disease remains unknown. However, the observation of (modest) reductions in (non-fatal) myocardial infarction in meta-analyses of clinical trials of various approaches to glucose-lowering in individuals with type 2 diabetes $^{26-28}$ and a small (although non-significant) reduction in a combined cardiovascular endpoint with intensive glucose-lowering treatment in screening-detected type 2 diabetes ${ }^{29}$ suggest that glucose itself may play a role.

\section{COMMON CAROTID INTIMA-MEDIA THICKNESS AS SURROGATE MARKER OF CARDIOVASCULAR DISEASE}

Atherosclerosis, a chronic inflammatory disease of the large arteries, is the major underlying cause of cardiovascular disease. ${ }^{30-32}$ It is primarily a process of the intima layer as can be appreciated in Figure 1.1, which shows a 'normal' artery with its intima, media and adventitia layers and an early atherosclerotic lesion. ${ }^{33}$ An initial lesion may progress into an advanced plaque with a fibrous cap overlying a necrotic core. The ongoing inflammatory response, matrix degradation and cell death may eventually thin this fibrous cap and increase the inflammatory and necrotic core content, thereby increasing the risk for plaque ruptures, which may eventually cause clinical complications such as myocardial infarctions and strokes. ${ }^{30,32}$

With the use of (non-invasive) ultrasound techniques, arterial wall characteristics such as wall thickness can be evaluated as tissues of different densities (such as the lumen and the arterial wall layers) reflect the emitted ultrasound waves differently. Although advanced techniques to process ultrasound recordings (by automatically analyzing these radiofrequency signals ${ }^{34}$ ) provide estimates four to eight times more precise than older ultrasound image analyses techniques, ${ }^{35}$ these still do not distinguish between the intima and media layers. Therefore, at present, the combined thickness of these layers [i.e. the intima-media thickness (IMT)] is widely recognized as a marker for atherosclerotic disease. 


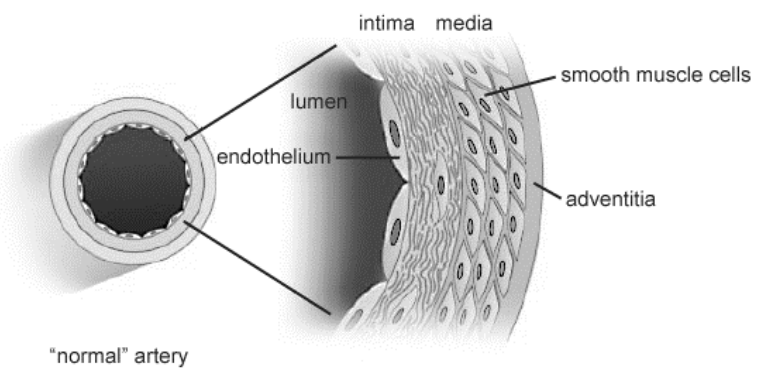

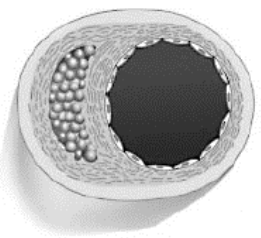

early atherosclerosis

Figure 1.1 Schematic representations of a 'normal' artery (with its intima, media and adventitia layers) and an artery with early atherosclerosis (figure adapted from Libby 2002, Nature).

The IMT of the carotid artery (CIMT) is most commonly measured, as it is a usual location for atherosclerosis and it is easily accessible for ultrasound measurements. In addition, in contrast to the more peripheral muscular arteries, the carotid artery is an elastic artery and the muscular media is relatively small. ${ }^{36}$ Increased CIMT is therefore considered to represent mainly intima thickening. ${ }^{37}$ Numerous studies have shown that these noninvasive measures of CIMT can be measured with high reproducibility. ${ }^{34,38-40}$ In addition, CIMT correlates well with major cardiovascular risk factors, ${ }^{41,42}$ prevalent cardiovascular disease $^{43,44}$ and severity of atherosclerosis in other vascular beds. ${ }^{45-48}$ Furthermore, CIMT predicts incident cardiovascular events ${ }^{49}$ and its progression over time may be decreased by targeted interventions. ${ }^{50-52}$ As such, CIMT is regarded a suitable surrogate marker for cardiovascular disease ${ }^{53}$ and is currently widely used for the pre-symptomatic detection of atherosclerotic disease and its progression in clinical and epidemiological studies, improving efficiency and aiming at decreasing follow-up time usually needed in studies with 'hard' cardiovascular endpoints. ${ }^{53-56}$

\section{THE GLYCATION PATHWAY}

Although the exact pathophysiological mechanisms explaining the increased risk for vascular diseases in individuals with diabetes are not completely understood, Brownlee ${ }^{10}$ was the first to propose a unifying mechanism to link four potential pathways of hyperglycaemia-induced (vascular) damage (figure 1.2). ${ }^{10}$

Due to hyperglycaemia, superoxide $\left(\mathrm{O}_{2}{ }^{\circ}\right)$ is excessively produced and partially inhibits the glycolytic enzyme glyceraldehyde-3-phosphate dehydrogenase (GAPDH), thereby diverting upstream metabolites from glycolysis into pathways of glucose 
overutilization. This results in increased flux through the polyol, hexosamine and protein kinase C (PKC) pathways. In addition, the glycation pathway, in which production of intracellular AGE precursors (such as methylglyoxal) is increased, results in increased production of AGEs. As the latter pathway is the focus of this thesis, the formation of AGEs, their pathophysiological consequences and possibilities for intervention in this pathway will be further introduced.

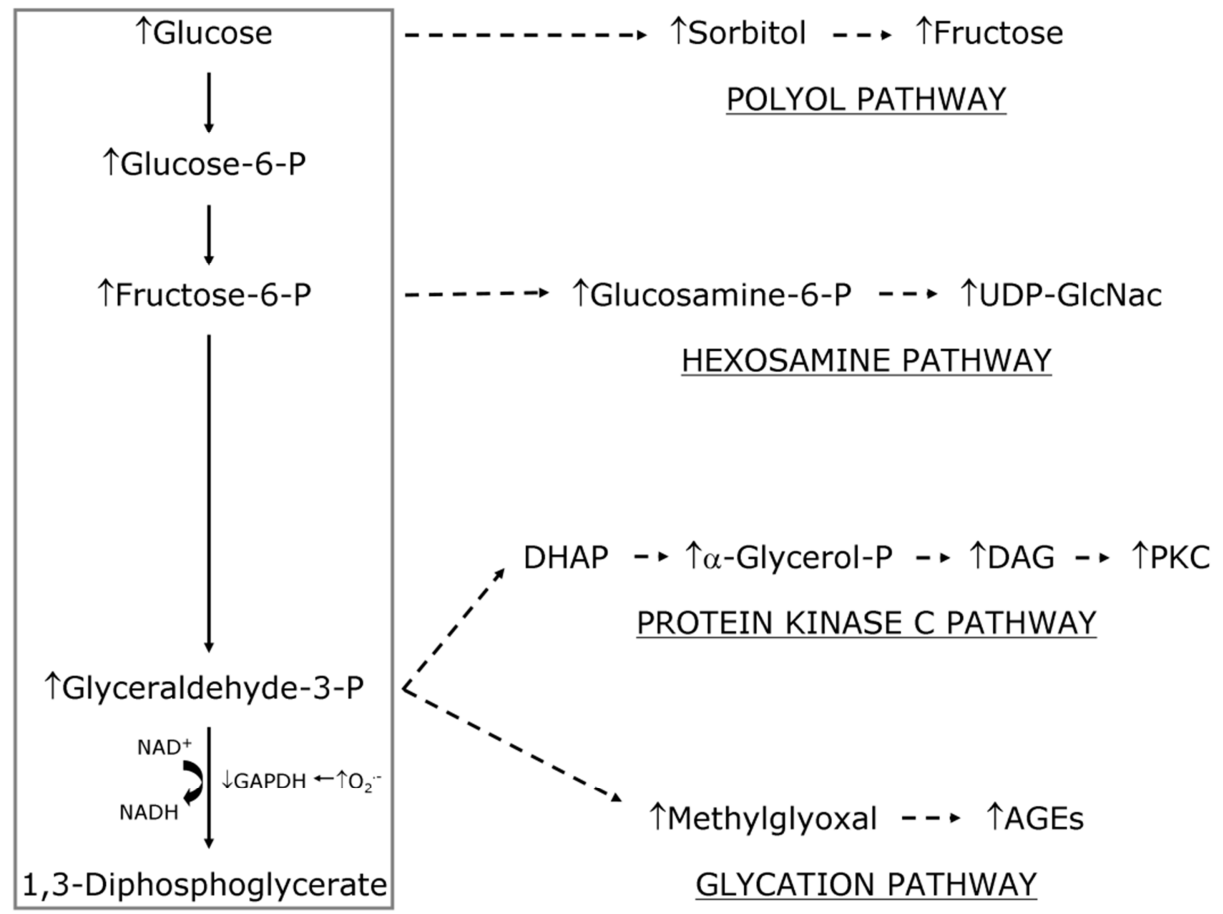

Figure 1.2 Four potential pathways of hyperglycaemia-induced damage (figure adapted from Brownlee 2001, Nature). DAG, diacylglycerol; DHAP, dihydroxyacetone phosphate; $\mathrm{P}$, phosphate; UDP-GIcNAc, uridine diphosphate-Nacetylglucosamine.

\section{FORMATION OF ADVANCED GLYCATION ENDPRODUCTS}

Non-enzymatic glycation, first described by Louis Camille Maillard in the early $1900 \mathrm{~s}^{57}$ involves the condensation reaction of the carbonyl group of sugars with the $\mathrm{N}$-terminus or free amino groups of proteins via a nucleophilic addition, resulting first in the rapid formation of a Schiff base. Through acid-base catalysis, this labile adduct then undergoes rearrangements to the more stable Amadori-product. ${ }^{58}$ During the lifetime of most cellular 
and plasma proteins, Amadori products, such as glycated haemoglobin (i.e. $\mathrm{HbA}_{1 \mathrm{c}}$ ) are in equilibrium with glucose and levels of Amadori products will therefore rise and fall depending on the glucose concentration. ${ }^{59}$ Only a small part of these relatively stable intermediate Amadori products undergoes further oxidative reactions and can give rise to irreversible AGEs such as $\mathrm{N}^{\varepsilon}$ (1-carboxymethyl)lysine (CML), pentosidine and pyrraline. ${ }^{58,60}$ Because of their slow formation, it was long believed that AGEs accumulate only on longlived extracellular proteins. However, nowadays research also focuses on an additional rapid extracellular AGE formation on short-lived proteins and intracellular AGE formation by reactive dicarbonyl compounds. ${ }^{61,62}$ Glycolytic intermediates such as dihydroxyacetone phosphate, glyceraldehyde-3-phosphate and the dicarbonyl compounds glyoxal, methylglyoxal and 3-deoxyglucosone, but also reactive intermediates resulting from the polyol pathway such as fructose, fructose-3-phosphate and also 3-deoxyglucosone substantially contribute to intracellular AGE formation through reactions with proteins. ${ }^{63}$ Among these reactive compounds, methylglyoxal is believed to be the most potent glycating agent ${ }^{64}$ and is mainly formed by conversion of glyceraldehyde-3-phosphate and dihydroxyacetone phosphate, derived from glucose metabolism. ${ }^{65}$ Methylglyoxal reacts with arginine and lysine residues in proteins and subsequently forms the AGEs methylglyoxal-derived hydroimidazolone $(\mathrm{MG}-\mathrm{H} 1)^{64}$ and $\mathrm{N}^{\varepsilon}\left(1\right.$-carboxyethyl)lysine (CEL), ${ }^{66}$ respectively. Glyoxal and 3DG also react with arginine and the reactions of glyoxal and 3DG with lysine residues result in the formation of CML and pyrraline, respectively. ${ }^{58,67}$

Besides reducing sugars, other sources may contribute to the modification of proteins, including lipids. ${ }^{68,69}$ Lipid peroxidation of polyunsaturated fatty acids results in the formation of lipid peroxides, decomposition of which may produce several reactive carbonyl compounds. The carbonyl compounds formed this way include both dicarbonyls identical to those formed from glucose-derived glycolytic intermediates (e.g. methylglyoxal and glyoxal) and specific lipid peroxidation-derived compounds (e.g. malondialdehyde and 4-hydroxynonenal). ${ }^{68,} 70$ Further reaction of these carbonyl compounds with amino acid residues (e.g. of lysine) may result in the formation of CML and CEL, which are therefore, besides AGEs, also considered advanced lipoxidation products (ALEs). ${ }^{68,69}$

\section{PATHOPHYSIOLOGICAL CONSEQUENCES OF THE GLYCATION PATHWAY}

It was not until 1980 that the pathophysiological significance of the glycation pathway emerged in medical science, in particular in relation to ageing and the aetiology of diabetic complications. ${ }^{71}$ Several mechanisms have been proposed by which the glycation pathway may lead to (diabetic) vascular complications (figure 1.3). ${ }^{10}$ 


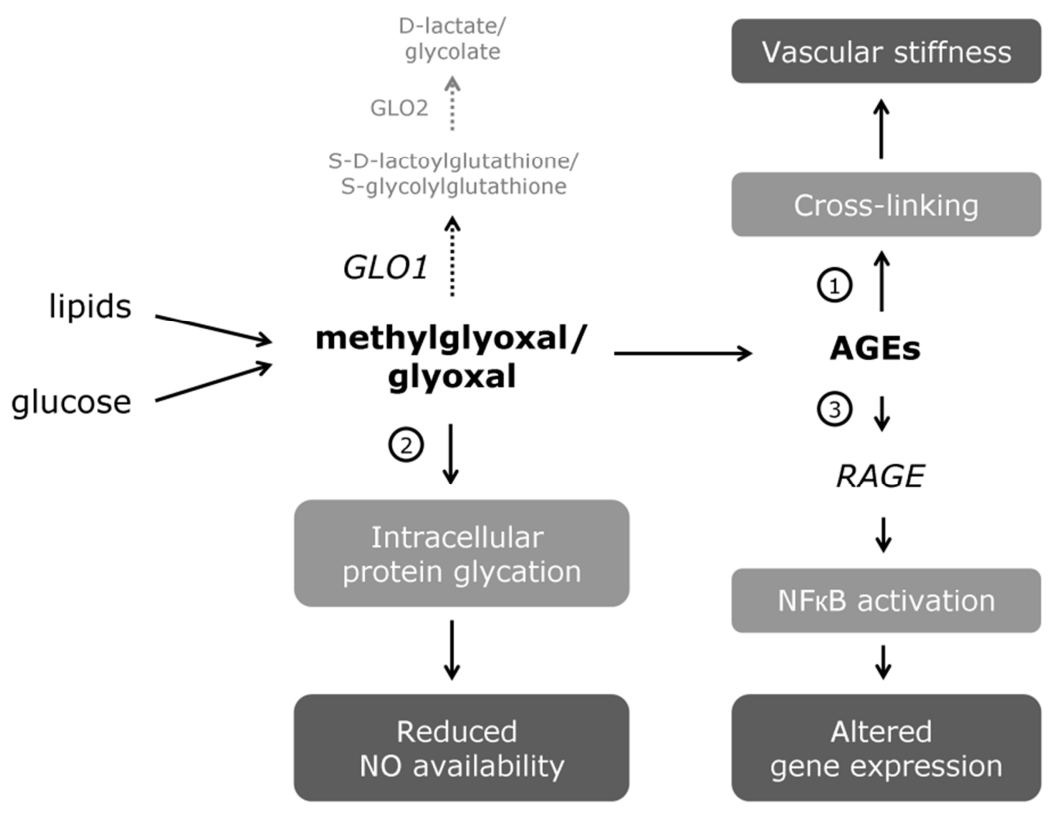

Figure 1.3 Three potential mechanisms of glycation-induced vascular damage

First, accumulation of AGEs in the extracellular matrix may cause aberrant cross-linking, resulting in a decrease of elasticity of vessels (i.e. arterial stiffness). ${ }^{72}$ Second, intracellular AGE formation (i.e. protein glycation) by reactive dicarbonyl compounds such as methylglyoxal and glyoxal may lead to quenching of nitric oxide and impaired function of growth factors. ${ }^{73}$ Third, AGEs may bind to AGE-receptors [such as the receptor for advanced glycation end products (RAGE)] on different cell types, which results in activation of key cell signalling pathways such as NF-KB activation with subsequent modulation of gene expression. $^{74,75}$

\section{DICARBONYL DETOXIFICATION: THE GLYOXALASE SYSTEM}

The dicarbonyl compounds methylglyoxal and glyoxal can be detoxified by the glyoxalase system. ${ }^{76-78}$ The rate limiting enzyme, glyoxalase 1 (GLO1), first converts, in the presence of reduced glutathione, methylglyoxal to S-D-lactoylglutathione, which is then converted to D-lactate by glyoxalase 2 (GLO2). ${ }^{76-78}$ Similarly, GLO1 and GLO2 convert glyoxal to Sglycolylglutathione and glycolate, respectively (Figure 1.3). ${ }^{78}$ Indeed, convincing experimental evidence demonstrated that GLO1 is the major cellular enzyme that catalyses the metabolism of methylglyoxal and has a critical role in suppressing the formation of AGEs. ${ }^{73,79}$ Additionally, in an in vivo (rat) model of diabetes, GLO1 
overexpression was shown to reduce not only blood levels of methylglyoxal and glyoxal and plasma $A G E s,{ }^{80}$ but also to improve endothelial function and attenuate renal impairment. ${ }^{81,82}$

Genetic variation in the gene coding for GLO1 may alter the expression and/or activity of GLO1. Several single nucleotide polymorphisms (SNPS) in the GLO1 gene have been described of which only one common variant exists within the coding sequence. ${ }^{83}$ Specifically, the GLO1 SNP rs2736654, which is a C/A substitution that causes an Ala111Glu change, has been found to influence enzyme activity so that the presence of the A-allele results in reduced GLO1 activity. ${ }^{84}$ The presence of the A allele has been found to be positively associated with prevalent cardiovascular and peripheral vascular disease ${ }^{85}$ and with overall mortality in haemodialysis patients, ${ }^{86}$ which may suggest a possible role for genetic variation in GLO1 in the development of cardiovascular disease. However, no associations had been found of this GLO1 SNP with CIMT and plaques ${ }^{87}$ or with prevalent nephropathy and retinopathy ${ }^{88}$ in individuals with type 2 diabetes.

\section{RECEPTOR FOR ADVANCED GLYCATION ENDPRODUCTS}

Out of the known receptors that can bind AGEs, RAGE has so far been mostly studied and best characterized. RAGE is a member of the immunoglobulin superfamily of cell surface molecules. ${ }^{89,90}$ In addition to binding with AGEs, RAGE interacts with other ligands such as high-mobility group box protein 1 and members of the S100/calgranulin family. ${ }^{91,} 92$ Binding to RAGE results in intracellular signalling which leads to activation of the proinflammatory transcription factor NF- $\kappa B$ and subsequent transcription of target genes, including pro-inflammatory cytokines and adhesion molecules ${ }^{93}$ in vascular cells such as endothelial cells, smooth muscle cells and macrophages. ${ }^{74,75,92}$

Genetic variation in the gene coding for RAGE (i.e. AGER) has been shown to alter the expression, activity and/or ligand binding affinity of RAGE. ${ }^{94}, 95$ Several SNPs in the RAGE gene have been described of which only one common variant exists within the coding sequence. Specifically, the RAGE SNP rs2070600, which causes a Gly82Ser substitution, has been found to influence RAGE binding affinity. ${ }^{94}$ In addition, the common SNPs 429T/C and -374T/A (in the RAGE promotor region) have been found to influence expression so that the presence of the $C$ and $A$ alleles, respectively, results in increased RAGE expression. ${ }^{95}$ These RAGE SNPs may as such affect the genetic susceptibility to vascular disease, though studies so far investigating the associations between RAGE SNPs and vascular disease have reported contradictory results. ${ }^{95-119}$ 


\section{POSSIBILITIES FOR INTERVENTION IN THE GLYCATION PATHWAY}

Given the deleterious effects of AGEs, agents with AGE inhibitory properties, which thereby may retard the development of complications are of interest. ${ }^{120}$ The burden of the glycation pathway may be decreased both by therapies that intervene in the production of AGEs - by specifically scavenging AGE precursors (i.e. dicarbonyl compounds) or by influencing mechanisms involved in AGE formation such as lipid peroxidation or oxidative stress - or by therapies that break vascular wall collagen cross-links. ${ }^{121-123}$ In the last decade, numerous studies have been published about compounds with potential AGE inhibitory effects. In particular the (AGE-inhibiting) effects of cross-link breakers, such as alagebrium (ALT-711), ${ }^{124-128}$ and the specific AGE inhibitor aminoguanidine ${ }^{129-134}$ have been extensively evaluated. In addition, it has been proposed that metformin, an oral antihyperglycaemic agent which enhances insulin sensitivity and lowers hepatic glucose output, may also have anti-glycation properties with a working mechanism similar to aminoguanidine due to a similarity in structure between both agents. ${ }^{135-137}$ Furthermore, blood pressure-lowering agents such as angiotensin receptor blockers (ARBs) and angiotensin converting enzyme ( $A C E$ ) inhibitors have been shown to attenuate the in vitro production of dicarbonyl compounds and $\mathrm{AGEs}^{138}$ due to their anti-oxidant ${ }^{139,140}$ and metal-chelating properties. ${ }^{139}$ Animal experiments have confirmed these effects as AGE accumulation in the kidneys ${ }^{140-146}$ and eyes ${ }^{147}$ was reduced after treatment with $A R B s^{140 \text {, }}$ ${ }^{142,144-147}$ or ACE inhibitors. ${ }^{141,143}$ However, the potential clinical value of AGE-inhibiting interventions remains to be established.

\section{OUTLINE OF THE THESIS}

The importance of the glycation pathway in the pathogenesis of diabetic vascular complications has been well established in numerous experimental studies, which have linked AGE accumulation in tissues to development of vascular complications and which have shown reductions in vascular complications with (specific) AGE-inhibiting interventions. However, epidemiological evidence on the role of the glycation pathway in type 2 diabetes and its micro- and macrovascular complications and efficacy of (specific) intervention to reduce the burden of the glycation pathway in humans is largely lacking.

In the first part of this thesis (Part 1), possibilities for interventions in the glycation pathway in individuals with and without type 2 diabetes were explored. Chapter 2 reviews the current clinical evidence in the literature on beneficial effects of specific AGE inhibitors and breakers and the potential AGE-inhibiting effects of therapies developed for purposes unrelated to the glycation pathway. The effects on circulating levels of AGEs and/or AGE 
precursors of interventions with the glucose-lowering agent metformin and the blood pressure-lowering agent irbesartan, as examined in two randomized trials, are described in Chapter 3 and Chapter 4, respectively.

In the second part of this thesis (Part 2), we investigated, in observational cohort studies, associations between measures of the glycation pathway and (markers of) cardiovascular disease. First, we established reference intervals for one of the markers of cardiovascular disease used (i.e. common CIMT), which are provided in Chapter 5. We then investigated whether polymorphisms in genes related to the glycation pathway were associated with (markers of) cardiovascular disease; Chapter 6 and Chapter 7 describe the results on the GLO1 and RAGE gene, respectively. We additionally investigated the associations of circulating levels of AGEs with (markers of) cardiovascular disease, which are given in Chapter 8. Finally, Chapter 9 summarizes and discusses the results of the studies presented in this thesis and gives some potential implications for future research.

\section{type 2 diabetes}

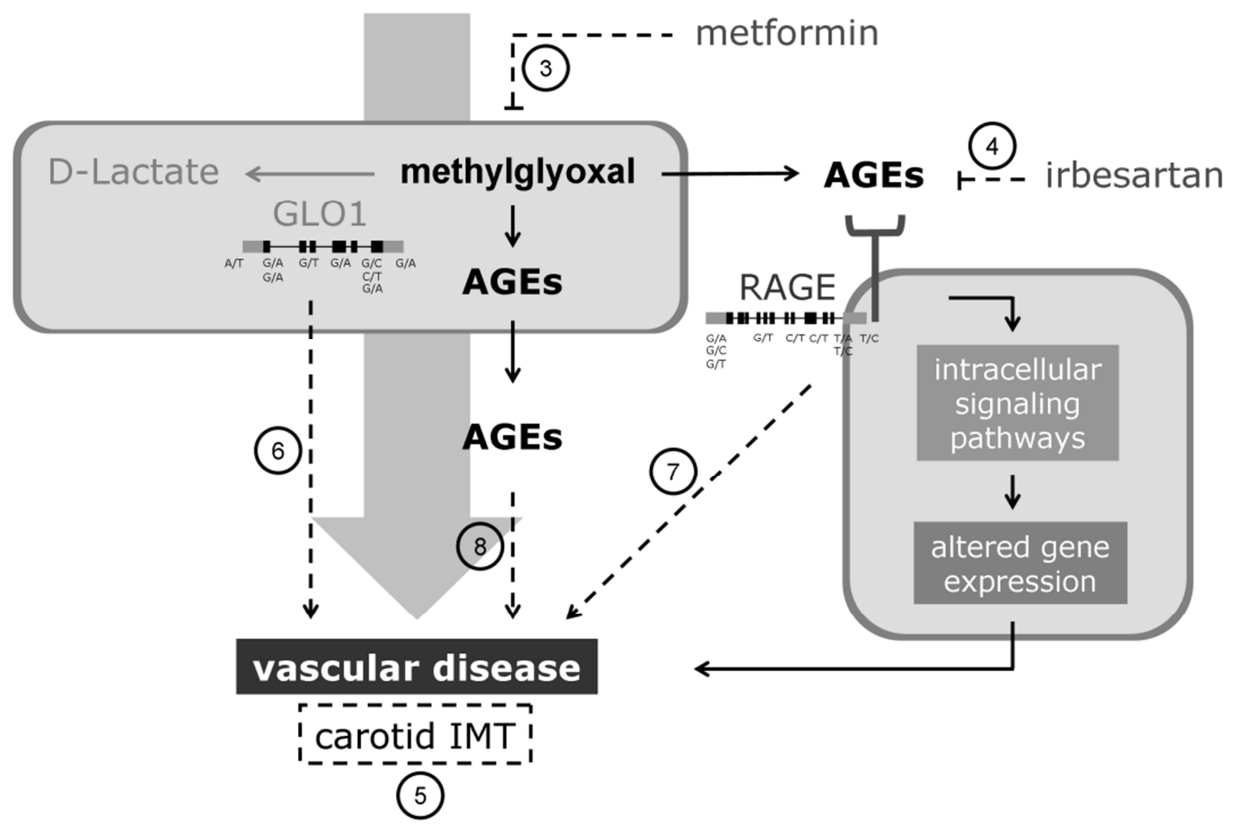

Figure 1.4 Schematic representation of the overall hypotheses investigated in this thesis. Dashed lines represent relations investigated and potential influences of drugs currently evaluated. Numbers 3-8 indicate the corresponding chapters. 


\section{REFERENCES}

1. World Health Organization. Diabetes: Fact sheet no. 312. 2011;Accessed February 2012

2. Danaei G, Finucane MM, Lu Y, Singh GM, Cowan MJ, Paciorek CJ, et al. National, regional, and global trends in fasting plasma glucose and diabetes prevalence since 1980: Systematic analysis of health examination surveys and epidemiological studies with 370 country-years and 2.7 million participants. Lancet. 2011;378:31-40

3. International Diabetes Federation. Idf diabetes atlas: The global burden, 5th edition [online]. 2011;Accessed February 2012

4. Baan CA, Schoemaker CG. Diabetes tot 2025. RIVM. 2009;Rapport 260322004/2009

5. The Emerging Risk Factors Collaboration, Sarwar N, Gao P, Seshasai SR, Gobin R, Kaptoge S, et al. Diabetes mellitus, fasting blood glucose concentration, and risk of vascular disease: A collaborative meta-analysis of 102 prospective studies. Lancet. 2010;375:2215-2222

6. The Emerging Risk Factors Collaboration, Seshasai SR, Kaptoge S, Thompson A, Di Angelantonio E, Gao P, et al. Diabetes mellitus, fasting glucose, and risk of cause-specific death. N Engl J Med. 2011;364:829-841

7. The Diabetes Control and Complications Trial Research Group. The effect of intensive treatment of diabetes on the development and progression of long-term complications in insulin-dependent diabetes mellitus. $N$ Engl J Med. 1993;329:977-986

8. UK Prospective Diabetes Study (UKPDS) Group. Intensive blood-glucose control with sulphonylureas or insulin compared with conventional treatment and risk of complications in patients with type 2 diabetes (ukpds 33) Lancet. 1998;352:837-853

9. Vlassara H, Palace MR. Diabetes and advanced glycation endproducts. J Intern Med. 2002;251:87-101

10. Brownlee M. Biochemistry and molecular cell biology of diabetic complications. Nature. 2001;414:813-820

11. Stitt AW. Advanced glycation: An important pathological event in diabetic and age related ocular disease. $\mathrm{Br}$ J Ophthalmol. 2001;85:746-753

12. American Diabetes Association. Diagnosis and classification of diabetes mellitus. Diabetes Care. 2004;27 Suppl 1:S5-S10

13. World Health Organization. Definition, diagnosis, and classification of diabetes mellitus and its complications. Report of a who consultation. Part 1: Diagnosis and classification of diabetes mellitus. Geneva, Switzerland: WHO; 1999.

14. World Health Organization. Definition and diagnosis of diabetes mellitus and intermediate hyperglycaemia: Report of a who/idf consultation. Geneva, Switzerland: WHO; 2006.

15. Sullivan PW, Morrato EH, Ghushchyan V, Wyatt HR, Hill JO. Obesity, inactivity, and the prevalence of diabetes and diabetes-related cardiovascular comorbidities in the u.S., 2000-2002. Diabetes Care. 2005;28:1599-1603

16. Zeggini E, Scott $\mathrm{L}$, Saxena R, Voight BF, Marchini IL, Hu T, et al. Meta-analysis of genome-wide association data and large-scale replication identifies additional susceptibility loci for type 2 diabetes. Nat Genet. 2008;40:638-645

17. Finucane MM, Stevens GA, Cowan MJ, Danaei G, Lin JK, Paciorek CJ, et al. National, regional, and global trends in body-mass index since 1980: Systematic analysis of health examination surveys and epidemiological studies with 960 country-years and 9.1 million participants. Lancet. 2011;377:557-567

18. Tobias M. Global control of diabetes: Information for action. Lancet. 2011;378:3-4

19. Chaturvedi N. The burden of diabetes and its complications: Trends and implications for intervention. Diabetes Res Clin Pract. 2007;76 Suppl 1:S3-12

20. Yau JW, Rogers SL, Kawasaki R, Lamoureux EL, Kowalski JW, Bek T, et al. Global prevalence and major risk factors of diabetic retinopathy. Diabetes Care. 2012

21. Adler Al, Stevens RJ, Manley SE, Bilous RW, Cull CA, Holman RR. Development and progression of nephropathy in type 2 diabetes: The united kingdom prospective diabetes study (ukpds 64). Kidney Int. 2003;63:225-232

22. Valmadrid $\mathrm{CT}$, Klein R, Moss $\mathrm{SE}$, Klein BE. The risk of cardiovascular disease mortality associated with microalbuminuria and gross proteinuria in persons with older-onset diabetes mellitus. Arch Intern Med. 2000;160:1093-1100 
23. Resnikoff S, Pascolini D, Etya'ale D, Kocur I, Pararajasegaram R, Pokharel GP, et al. Global data on visual impairment in the year 2002. Bull World Health Organ. 2004;82:844-851

24. White SL, Chadban SJ, Jan S, Chapman JR, Cass A. How can we achieve global equity in provision of renal replacement therapy? Bull World Health Organ. 2008;86:229-237

25. Sheetz MJ, King GL. Molecular understanding of hyperglycemia's adverse effects for diabetic complications. JAMA. 2002;288:2579-2588

26. Boussageon R, Bejan-Angoulvant T, Saadatian-Elahi M, Lafont S, Bergeonneau C, Kassai B, et al. Effect of intensive glucose lowering treatment on all cause mortality, cardiovascular death, and microvascular events in type 2 diabetes: Meta-analysis of randomised controlled trials. Bmj. 2011;343:d4169

27. Turnbull FM, Abraira C, Anderson RJ, Byington RP, Chalmers JP, Duckworth WC, et al. Intensive glucose control and macrovascular outcomes in type 2 diabetes. Diabetologia. 2009;52:2288-2298

28. Ray KK, Seshasai SR, Wijesuriya S, Sivakumaran R, Nethercott S, Preiss D, et al. Effect of intensive control of glucose on cardiovascular outcomes and death in patients with diabetes mellitus: A meta-analysis of randomised controlled trials. Lancet. 2009;373:1765-1772

29. Griffin SJ, Borch-Johnsen K, Davies MJ, Khunti K, Rutten GE, Sandbaek A, et al. Effect of early intensive multifactorial therapy on 5-year cardiovascular outcomes in individuals with type 2 diabetes detected by screening (addition-europe): A cluster-randomised trial. Lancet. 2011;378:156-167

30. Hansson GK. Inflammation, atherosclerosis, and coronary artery disease. N Engl J Med. 2005;352:1685-1695

31. Libby P, Ridker PM, Hansson GK. Progress and challenges in translating the biology of atherosclerosis. Nature. 2011;473:317-325

32. Sluimer JC, Daemen MJ. Novel concepts in atherogenesis: Angiogenesis and hypoxia in atherosclerosis. J Pathol. 2009;218:7-29

33. Libby P. Inflammation in atherosclerosis. Nature. 2002;420:868-874

34. Hoeks AP, Willekes C, Boutouyrie P, Brands PJ, Willigers JM, Reneman RS. Automated detection of local artery wall thickness based on m-line signal processing. Ultrasound Med Biol. 1997;23:1017-1023

35. Laurent S, Cockcroft J, Van Bortel L, Boutouyrie P, Giannattasio C, Hayoz D, et al. Expert consensus document on arterial stiffness: Methodological issues and clinical applications. Eur Heart J. 2006;27:25882605

36. Van Bortel LM. What does intima-media thickness tell us? J Hypertens. 2005;23:37-39

37. Grobbee DE, Bots ML. Carotid artery intima-media thickness as an indicator of generalized atherosclerosis. $J$ Intern Med. 1994;236:567-573

38. Kanters SD, Algra A, van Leeuwen MS, Banga JD. Reproducibility of in vivo carotid intima-media thickness measurements: A review. Stroke. 1997;28:665-671

39. Kanters SD, Elgersma OE, Banga JD, van Leeuwen MS, Algra A. Reproducibility of measurements of intimamedia thickness and distensibility in the common carotid artery. Eur J Vasc Endovasc Surg. 1998;16:28-35

40. Bots ML, Mulder PG, Hofman A, van Es GA, Grobbee DE. Reproducibility of carotid vessel wall thickness measurements. The rotterdam study. J Clin Epidemiol. 1994;47:921-930

41. Baldassarre D, Nyyssonen K, Rauramaa R, de Faire U, Hamsten A, Smit AJ, et al. Cross-sectional analysis of baseline data to identify the major determinants of carotid intima-media thickness in a european population: The improve study. Eur Heart J. 2010;31:614-622

42. Sinning C, Wild PS, Echevarria FM, Wilde S, Schnabel R, Lubos E, et al. Sex differences in early carotid atherosclerosis (from the community-based gutenberg-heart study). Am J Cardiol. 2011;107:1841-1847

43. Geroulakos G, O'Gorman DJ, Kalodiki E, Sheridan DJ, Nicolaides AN. The carotid intima-media thickness as a marker of the presence of severe symptomatic coronary artery disease. Eur Heart J. 1994;15:781-785

44. Ebrahim S, Papacosta O, Whincup P, Wannamethee G, Walker M, Nicolaides AN, et al. Carotid plaque, intima media thickness, cardiovascular risk factors, and prevalent cardiovascular disease in men and women: The british regional heart study. Stroke. 1999;30:841-850

45. Bots ML, Hofman A, De Jong PT, Grobbee DE. Common carotid intima-media thickness as an indicator of atherosclerosis at other sites of the carotid artery. The rotterdam study. Ann Epidemiol. 1996;6:147-153

46. Bots ML, Witteman JC, Grobbee DE. Carotid intima-media wall thickness in elderly women with and without atherosclerosis of the abdominal aorta. Atherosclerosis. 1993;102:99-105

47. Bots ML, Baldassarre D, Simon A, de Groot E, O'Leary DH, Riley W, et al. Carotid intima-media thickness and coronary atherosclerosis: Weak or strong relations? Eur Heart J. 2007;28:398-406

48. Allan PL, Mowbray PI, Lee AJ, Fowkes FG. Relationship between carotid intima-media thickness and symptomatic and asymptomatic peripheral arterial disease. The edinburgh artery study. Stroke. $1997 ; 28: 348-353$ 
49. Lorenz MW, Markus HS, Bots ML, Rosvall M, Sitzer M. Prediction of clinical cardiovascular events with carotid intima-media thickness: A systematic review and meta-analysis. Circulation. 2007;115:459-467

50. Bedi US, Singh M, Singh PP, Bhuriya R, Bahekar A, Molnar J, et al. Effects of statins on progression of carotid atherosclerosis as measured by carotid intimal--medial thickness: A meta-analysis of randomized controlled trials. J Cardiovasc Pharmacol Ther. 2010;15:268-273

51. Wang JG, Staessen JA, Li Y, Van Bortel LM, Nawrot T, Fagard R, et al. Carotid intima-media thickness and antihypertensive treatment: A meta-analysis of randomized controlled trials. Stroke. 2006;37:1933-1940

52. Polak JF, Backlund JY, Cleary PA, Harrington AP, O'Leary DH, Lachin JM, et al. Progression of carotid artery intima-media thickness during 12 years in the diabetes control and complications trial/epidemiology of diabetes interventions and complications (dcct/edic) study. Diabetes. 2011;60:607-613

53. Peters SA, Grobbee DE, Bots ML. Carotid intima-media thickness: A suitable alternative for cardiovascular risk as outcome? Eur J Cardiovasc Prev Rehabil. 2011;18:167-174

54. Bots ML, Evans GW, Riley WA, Grobbee DE. Carotid intima-media thickness measurements in intervention studies: Design options, progression rates, and sample size considerations: A point of view. Stroke. 2003;34:2985-2994

55. Touboul PJ, Hennerici MG, Meairs S, Adams H, Amarenco P, Bornstein N, et al. Mannheim carotid intimamedia thickness consensus (2004-2006). An update on behalf of the advisory board of the 3rd and 4th watching the risk symposium, 13th and 15th european stroke conferences, mannheim, germany, 2004, and brussels, belgium, 2006. Cerebrovasc Dis. 2007;23:75-80

56. Urbina EM, Williams RV, Alpert BS, Collins RT, Daniels SR, Hayman L, et al. Noninvasive assessment of subclinical atherosclerosis in children and adolescents: Recommendations for standard assessment for clinical research: A scientific statement from the american heart association. Hypertension. 2009;54:919950

57. Maillard LC. Action de acides aminés sur les sucres: Formation des mélanoïdines par voie méthodique. $C R$ Acad Sci 1912;154:66-68

58. Singh R, Barden A, Mori T, Beilin L. Advanced glycation end-products: A review. Diabetologia. 2001;44:129146

59. Goldstein DE, Little RR, Lorenz RA, Malone JI, Nathan D, Peterson CM, et al. Tests of glycemia in diabetes. Diabetes Care. 2004;27:1761-1773

60. Monnier VM, Cerami A. Nonenzymatic browning in vivo: Possible process for aging of long-lived proteins. Science. 1981;211:491-493

61. Giardino I, Edelstein D, Brownlee M. Nonenzymatic glycosylation in vitro and in bovine endothelial cells alters basic fibroblast growth factor activity. A model for intracellular glycosylation in diabetes. J Clin Invest. 1994;94:110-117

62. Giardino I, Edelstein D, Brownlee M. Bcl-2 expression or antioxidants prevent hyperglycemia-induced formation of intracellular advanced glycation endproducts in bovine endothelial cells. J Clin Invest. 1996;97:1422-1428

63. Schalkwijk CG, Stehouwer CD, van Hinsbergh VW. Fructose-mediated non-enzymatic glycation: Sweet coupling or bad modification. Diabetes Metab Res Rev. 2004;20:369-382

64. Westwood ME, Thornalley PJ. Molecular characteristics of methylglyoxal-modified bovine and human serum albumins. Comparison with glucose-derived advanced glycation endproduct-modified serum albumins. J Protein Chem. 1995;14:359-372

65. Phillips SA, Thornalley PJ. Formation of methylglyoxal and d-lactate in human red blood cells in vitro. Biochem Soc Trans. 1993;21:163S

66. Ahmed MU, Brinkmann Frye E, Degenhardt TP, Thorpe SR, Baynes JW. N-epsilon-(carboxyethyl)lysine, a product of the chemical modification of proteins by methylglyoxal, increases with age in human lens proteins. Biochem J. 1997;324 ( Pt 2):565-570

67. Niwa T. 3-deoxyglucosone: Metabolism, analysis, biological activity, and clinical implication. J Chromatogr B Biomed Sci Appl. 1999;731:23-36

68. Baynes JW, Thorpe SR. Glycoxidation and lipoxidation in atherogenesis. Free Radic Biol Med. 2000;28:17081716

69. Fu MX, Requena JR, Jenkins AJ, Lyons TJ, Baynes JW, Thorpe SR. The advanced glycation end product, nepsilon-(carboxymethyl)lysine, is a product of both lipid peroxidation and glycoxidation reactions. J Biol Chem. 1996;271:9982-9986

70. Januszewski AS, Alderson NL, Jenkins AJ, Thorpe SR, Baynes JW. Chemical modification of proteins during peroxidation of phospholipids. J Lipid Res. 2005;46:1440-1449 
71. Monnier VM, Stevens VJ, Cerami A. Maillard reactions involving proteins and carbohydrates in vivo: Relevance to diabetes mellitus and aging. Prog Food Nutr Sci. 1981;5:315-327

72. Aronson D. Cross-linking of glycated collagen in the pathogenesis of arterial and myocardial stiffening of aging and diabetes. J Hypertens. 2003;21:3-12

73. Shinohara M, Thornalley PJ, Giardino I, Beisswenger P, Thorpe SR, Onorato J, et al. Overexpression of glyoxalase-i in bovine endothelial cells inhibits intracellular advanced glycation endproduct formation and prevents hyperglycemia-induced increases in macromolecular endocytosis. J Clin Invest. 1998;101:11421147

74. Miyazaki A, Nakayama H, Horiuchi S. Scavenger receptors that recognize advanced glycation end products. Trends Cardiovasc Med. 2002;12:258-262

75. Stern DM, Yan SD, Yan SF, Schmidt AM. Receptor for advanced glycation endproducts (rage) and the complications of diabetes. Ageing Res Rev. 2002;1:1-15

76. Thornalley PJ. Glyoxalase i--structure, function and a critical role in the enzymatic defence against glycation. Biochem Soc Trans. 2003;31:1343-1348

77. Rabbani N, Thornalley PJ. Glyoxalase in diabetes, obesity and related disorders. Semin Cell Dev Biol. 2011;22:309-317

78. Thornalley PJ. Glutathione-dependent detoxification of alpha-oxoaldehydes by the glyoxalase system: Involvement in disease mechanisms and antiproliferative activity of glyoxalase i inhibitors. Chem Biol Interact. 1998;111-112:137-151

79. Kalapos MP. Methylglyoxal in living organisms: Chemistry, biochemistry, toxicology and biological implications. Toxicol Lett. 1999;110:145-175

80. Brouwers O, Niessen PM, Ferreira I, Miyata T, Scheffer PG, Teerlink T, et al. Overexpression of glyoxalase-i reduces hyperglycemia-induced levels of advanced glycation end products and oxidative stress in diabetic rats. J Biol Chem. 2011;286:1374-1380

81. Brouwers O, Niessen PMG, Miyata T, Østergaard J, Flyvbjerg A, Peutz-Kootstra CJ, et al. Glyoxalase-i overexpression attenuates early renal impairment in diabetic rats. Diabetologia. 2011;54:S44

82. Brouwers O, Niessen PMG, Miyata T, Teerlink T, Janssen BJ, De Mey JGR, et al. Overexpression of glyoxalase-i improves vascular function in a rat model of diabetes. Diabetologia. 2010;53:S520

83. Gale CP, Grant PJ. The characterisation and functional analysis of the human glyoxalase-1 gene using methods of bioinformatics. Gene. 2004;340:251-260

84. Barua M, Jenkins EC, Chen W, Kuizon S, Pullarkat RK, Junaid MA. Glyoxalase i polymorphism rs2736654 causing the ala111glu substitution modulates enzyme activity--implications for autism. Autism Res. 2011;4:262-270

85. Kalousova M, Germanova A, Jachymova M, Mestek O, Tesav V, Zima T. A419c (e111a) polymorphism of the glyoxalase $\mathrm{i}$ gene and vascular complications in chronic hemodialysis patients. Ann $N$ Y Acad Sci. 2008;1126:268-271

86. Kalousova M, Jachymova M, Germanova A, Kubena AA, Tesar V, Zima T. Genetic predisposition to advanced glycation end products toxicity is related to prognosis of chronic hemodialysis patients. Kidney Blood Press Res. 2010;33:30-36

87. Wu JC, Li XH, Wang JB, Tang JF, Wang YF, Peng YD. Glyoxalase i and aldose reductase gene polymorphisms and susceptibility to carotid atherosclerosis in type 2 diabetes. Genet Test Mol Biomarkers. 2011;15:273279

88. Wu JC, Li XH, Peng YD, Wang JB, Tang JF, Wang YF. Association of two glyoxalase i gene polymorphisms with nephropathy and retinopathy in type 2 diabetes. J Endocrinol Invest. 2011;34:e343-348

89. Schmidt AM, Vianna M, Gerlach M, Brett J, Ryan J, Kao J, et al. Isolation and characterization of two binding proteins for advanced glycosylation end products from bovine lung which are present on the endothelial cell surface. J Biol Chem. 1992;267:14987-14997

90. Neeper M, Schmidt AM, Brett J, Yan SD, Wang F, Pan YC, et al. Cloning and expression of a cell surface receptor for advanced glycosylation end products of proteins. J Biol Chem. 1992;267:14998-15004

91. Schmidt AM, Yan SD, Yan SF, Stern DM. The biology of the receptor for advanced glycation end products and its ligands. Biochim Biophys Acta. 2000;1498:99-111

92. Bierhaus A, Humpert PM, Morcos M, Wendt T, Chavakis T, Arnold B, et al. Understanding rage, the receptor for advanced glycation end products. J Mol Med (Berl). 2005;83:876-886

93. Barnes PJ, Karin M. Nuclear factor-kappab: A pivotal transcription factor in chronic inflammatory diseases. $N$ Engl J Med. 1997;336:1066-1071 
94. Hofmann MA, Drury S, Hudson BI, Gleason MR, Qu W, Lu Y, et al. Rage and arthritis: The g82s polymorphism amplifies the inflammatory response. Genes Immun. 2002;3:123-135

95. Hudson BI, Stickland MH, Futers TS, Grant PJ. Effects of novel polymorphisms in the rage gene on transcriptional regulation and their association with diabetic retinopathy. Diabetes. 2001;50:1505-1511

96. dos Santos KG, Canani LH, Gross JL, Tschiedel B, Pires Souto KE, Roisenberg I. The -374a allele of the receptor for advanced glycation end products gene is associated with a decreased risk of ischemic heart disease in african-brazilians with type 2 diabetes. Mol Genet Metab. 2005;85:149-156

97. Lindholm E, Bakhtadze E, Sjogren M, Cilio CM, Agardh E, Groop L, et al. The -374 t/a polymorphism in the gene encoding rage is associated with diabetic nephropathy and retinopathy in type 1 diabetic patients. Diabetologia. 2006;49:2745-2755

98. Pettersson-Fernholm K, Forsblom C, Hudson BI, Perola M, Grant PJ, Groop PH. The functional -374 t/a rage gene polymorphism is associated with proteinuria and cardiovascular disease in type 1 diabetic patients. Diabetes. 2003;52:891-894

99. Picheth G, Costantini CO, Pedrosa FO, Leme da Rocha Martinez T, Maltempi de Souza E. The -374a allele of the receptor for advanced glycation end products (rage) gene promoter is a protective factor against cardiovascular lesions in type 2 diabetes mellitus patients. Clin Chem Lab Med. 2007;45:1268-1272

100. Poirier O, Nicaud V, Vionnet N, Raoux S, Tarnow L, Vlassara H, et al. Polymorphism screening of four genes encoding advanced glycation end-product putative receptors. Association study with nephropathy in type 1 diabetic patients. Diabetes. 2001;50:1214-1218

101. Shim CY, Park S, Yoon SJ, Park HY, Kim HT, Oh B, et al. Association of rage gene polymorphisms with in-stent restenosis in non-diabetic korean population. Cardiology. 2007;107:261-268

102. Yoon SJ, Park S, Shim CY, Park CM, Ko YG, Choi D, et al. Association of rage gene polymorphisms with coronary artery disease in the korean population. Coron Artery Dis. 2007;18:1-8

103. Zee RY, Romero JR, Gould JL, Ricupero DA, Ridker PM. Polymorphisms in the advanced glycosylation end product-specific receptor gene and risk of incident myocardial infarction or ischemic stroke. Stroke. 2006;37:1686-1690

104. Falcone C, Campo I, Emanuele E, Buzzi MP, Geroldi D, Belvito C, et al. $-374 \mathrm{t} / \mathrm{a}$ polymorphism of the rage gene promoter in relation to severity of coronary atherosclerosis. Clin Chim Acta. 2005;354:111-116

105. Falcone C, Geroldi D, Buzzi MP, Emanuele E, Yilmaz Y, Fontana JM, et al. The -374t/a rage polymorphism protects against future cardiac events in nondiabetic patients with coronary artery disease. Arch Med Res. 2008;39:320-325

106. Globocnik Petrovic M, Steblovnik K, Peterlin B, Petrovic D. The - 429 t/c and - 374 t/a gene polymorphisms of the receptor of advanced glycation end products gene are not risk factors for diabetic retinopathy in caucasians with type 2 diabetes. Klin Monatsbl Augenheilkd. 2003;220:873-876

107. Hofmann MA, Yang Q, Harja E, Kedia P, Gregersen PK, Cupples LA, et al. The rage gly82ser polymorphism is not associated with cardiovascular disease in the framingham offspring study. Atherosclerosis. 2005;182:301-305

108. JiXiong X, BiLin X, MingGong Y, ShuQin L. -429t/c and -374t/a polymorphisms of rage gene promoter are not associated with diabetic retinopathy in chinese patients with type 2 diabetes. Diabetes Care. 2003;26:26962697

109. Kirbis J, Milutinovic A, Steblovnik K, Teran N, Terzic R, Zorc M. The -429 t/c and -374 t/a gene polymorphisms of the receptor of advanced glycation end products gene (rage) are not risk factors for coronary artery disease in slovene population with type 2 diabetes. Coll Antropol. 2004;28:611-616

110. Lindholm E, Bakhtadze E, Cilio C, Agardh E, Groop L, Agardh CD. Association between Ita, tnf and ager polymorphisms and late diabetic complications. PLoS ONE. 2008;3:e2546

111. Prevost G, Fajardy I, Besmond C, Balkau B, Tichet J, Fontaine P, et al. Polymorphisms of the receptor of advanced glycation endproducts (rage) and the development of nephropathy in type 1 diabetic patients. Diabetes Metab. 2005;31:35-39

112. Pulkkinen A, Viitanen L, Kareinen A, Lehto S, Laakso M. Gly82ser polymorphism of the receptor of advanced glycation end product gene is not associated with coronary heart disease in finnish nondiabetic subjects or in patients with type 2 diabetes. Diabetes Care. 2000;23:864

113. Ramprasad S, Radha V, Mathias RA, Majumder PP, Rao MR, Rema M. Rage gene promoter polymorphisms and diabetic retinopathy in a clinic-based population from south india. Eye. 2007;21:395-401

114. Yoshioka K, Yoshida T, Takakura Y, Umekawa T, Kogure A, Toda H, et al. Association study of g1704t and g82s polymorphisms of rage gene for microalbuminuria in japanese type 2 diabetic patients. Metabolism. 2005;54:488-491 
115. Yoshioka K, Yoshida T, Takakura Y, Umekawa T, Kogure A, Toda H, et al. Relation between polymorphisms g1704t and g82s of rage gene and diabetic retinopathy in japanese type 2 diabetic patients. Intern Med. 2005;44:417-421

116. Kankova K, Beranek M, Hajek D, Vlkova E. Polymorphisms 1704g/t, 2184a/g, and 2245g/a in the rage gene are not associated with diabetic retinopathy in niddm: Pilot study. Retina. 2002;22:119-121

117. Kumaramanickavel G, Ramprasad VL, Sripriya S, Upadyay NK, Paul PG, Sharma T. Association of gly82ser polymorphism in the rage gene with diabetic retinopathy in type ii diabetic asian indian patients. J Diabetes Complications. 2002;16:391-394

118. Liu L, Xiang K. Rage gly82ser polymorphism in diabetic microangiopathy. Diabetes Care. 1999;22:646

119. Matsunaga-Irie S, Maruyama T, Yamamoto Y, Motohashi Y, Hirose H, Shimada A, et al. Relation between development of nephropathy and the p22phox c242t and receptor for advanced glycation end product g1704t gene polymorphisms in type 2 diabetic patients. Diabetes Care. 2004;27:303-307

120. Monnier VM. Intervention against the maillard reaction in vivo. Arch Biochem Biophys. 2003;419:1-15

121. Peyroux J, Sternberg M. Advanced glycation endproducts (ages): Pharmacological inhibition in diabetes. Pathol Biol (Paris). 2006;54:405-419

122. Schalkwijk CG. Therapeutic interventions in the glyc(oxid)ation pathway. Immun Endoc \& Metab Agents in Med Chem. 2007; 7:57-68

123. Sourris KC, Harcourt BE, Forbes JM. A new perspective on therapeutic inhibition of advanced glycation in diabetic microvascular complications: Common downstream endpoints achieved through disparate therapeutic approaches? Am J Nephrol. 2009;30:323-335

124. Asif M, Egan J, Vasan S, Jyothirmayi GN, Masurekar MR, Lopez S, et al. An advanced glycation endproduct cross-link breaker can reverse age-related increases in myocardial stiffness. Proc Natl Acad Sci U S A. 2000;97:2809-2813

125. Candido R, Forbes JM, Thomas MC, Thallas V, Dean RG, Burns WC, et al. A breaker of advanced glycation end products attenuates diabetes-induced myocardial structural changes. Circ Res. 2003;92:785-792

126. Vasan S, Zhang X, Zhang X, Kapurniotu A, Bernhagen J, Teichberg S, et al. An agent cleaving glucose-derived protein crosslinks in vitro and in vivo. Nature. 1996;382:275-278

127. Forbes JM, Yee LT, Thallas V, Lassila M, Candido R, Jandeleit-Dahm KA, et al. Advanced glycation end product interventions reduce diabetes-accelerated atherosclerosis. Diabetes. 2004;53:1813-1823

128. Wolffenbuttel BH, Boulanger CM, Crijns FR, Huijberts MS, Poitevin P, Swennen GN, et al. Breakers of advanced glycation end products restore large artery properties in experimental diabetes. Proc Natl Acad Sci U S A. 1998;95:4630-4634

129. Thornalley PJ. Use of aminoguanidine (pimagedine) to prevent the formation of advanced glycation endproducts. Arch Biochem Biophys. 2003;419:31-40

130. Brownlee M, Vlassara H, Kooney A, Ulrich P, Cerami A. Aminoguanidine prevents diabetes-induced arterial wall protein cross-linking. Science. 1986;232:1629-1632

131. Huijberts MS, Wolffenbuttel BH, Boudier HA, Crijns FR, Kruseman AC, Poitevin P, et al. Aminoguanidine treatment increases elasticity and decreases fluid filtration of large arteries from diabetic rats. J Clin Invest. 1993;92:1407-1411

132. Edelstein D, Brownlee M. Aminoguanidine ameliorates albuminuria in diabetic hypertensive rats. Diabetologia. 1992;35:96-97

133. Kern TS, Engerman RL. Pharmacological inhibition of diabetic retinopathy: Aminoguanidine and aspirin. Diabetes. 2001;50:1636-1642

134. Schmidt RE, Dorsey DA, Beaudet LN, Reiser KM, Williamson JR, Tilton RG. Effect of aminoguanidine on the frequency of neuroaxonal dystrophy in the superior mesenteric sympathetic autonomic ganglia of rats with streptozocin-induced diabetes. Diabetes. 1996;45:284-290

135. Ruggiero-Lopez D, Lecomte M, Moinet G, Patereau G, Lagarde M, Wiernsperger N. Reaction of metformin with dicarbonyl compounds. Possible implication in the inhibition of advanced glycation end product formation. Biochem Pharmacol. 1999;58:1765-1773

136. Beisswenger P, Ruggiero-Lopez D. Metformin inhibition of glycation processes. Diabetes Metab. 2003;29:6S95-103

137. Rabbani N, Chittari MV, Bodmer CW, Zehnder D, Ceriello A, Thornalley PJ. Increased glycation and oxidative damage to apolipoprotein b100 of Idl cholesterol in patients with type 2 diabetes and effect of metformin. Diabetes. 2010;59:1038-1045 
138. Miyata T, van Ypersele de Strihou C, Ueda Y, Ichimori K, Inagi R, Onogi H, et al. Angiotensin ii receptor antagonists and angiotensin-converting enzyme inhibitors lower in vitro the formation of advanced glycation end products: Biochemical mechanisms. J Am Soc Nephrol. 2002;13:2478-2487

139. Miyata T, van Ypersele de Strihou C. Angiotensin ii receptor blockers and angiotensin converting enzyme inhibitors: Implication of radical scavenging and transition metal chelation in inhibition of advanced glycation end product formation. Arch Biochem Biophys. 2003;419:50-54

140. Izuhara Y, Sada T, Yanagisawa H, Koike H, Ohtomo S, Dan T, et al. A novel sartan derivative with very low angiotensin ii type 1 receptor affinity protects the kidney in type 2 diabetic rats. Arterioscler Thromb Vasc Biol. 2008;28:1767-1773

141. Forbes JM, Cooper ME, Thallas V, Burns WC, Thomas MC, Brammar GC, et al. Reduction of the accumulation of advanced glycation end products by ace inhibition in experimental diabetic nephropathy. Diabetes. 2002;51:3274-3282

142. Forbes JM, Thomas MC, Thorpe SR, Alderson NL, Cooper ME. The effects of valsartan on the accumulation of circulating and renal advanced glycation end products in experimental diabetes. Kidney Int Suppl. 2004:S105-107

143. Liu XP, Pang YJ, Zhu WW, Zhao TT, Zheng M, Wang YB, et al. Benazepril, an angiotensin-converting enzyme inhibitor, alleviates renal injury in spontaneously hypertensive rats by inhibiting advanced glycation endproduct-mediated pathways. Clin Exp Pharmacol Physiol. 2009;36:287-296

144. Nangaku M, Miyata T, Sada T, Mizuno M, Inagi R, Ueda $Y$, et al. Anti-hypertensive agents inhibit in vivo the formation of advanced glycation end products and improve renal damage in a type 2 diabetic nephropathy rat model. J Am Soc Nephrol. 2003;14:1212-1222

145. Fan Q, Liao J, Kobayashi M, Yamashita M, Gu L, Gohda T, et al. Candesartan reduced advanced glycation end-products accumulation and diminished nitro-oxidative stress in type 2 diabetic kk/ta mice. Nephrol Dial Transplant. 2004;19:3012-3020

146. Thomas MC, Tikellis C, Burns WM, Bialkowski K, Cao Z, Coughlan MT, et al. Interactions between renin angiotensin system and advanced glycation in the kidney. J Am Soc Nephrol. 2005;16:2976-2984

147. Sugiyama T, Okuno T, Fukuhara M, Oku H, Ikeda T, Obayashi H, et al. Angiotensin ii receptor blocker inhibits abnormal accumulation of advanced glycation end products and retinal damage in a rat model of type 2 diabetes. Exp Eye Res. 2007;85:406-41 
PART $_{\text {P }}$ PT I

\section{INTERVENTIONS IN THE SLLCATION PATHWAY}





\section{CHAPTER 2}

CURRENT INTERYENTIONS IN THE SLYCATION PATHWAY EVIDENCE FROM CLINICAL STUDIES

LIAM ENGGELEM, COEN DA STEHOUWER, CASPER Ŝ SCHALKWIJK 


\section{ABSTRACT}

Increased formation of advanced glycation endproducts (AGEs) constitutes a potential mechanism of hyperglycaemia-induced micro- and macrovascular disease in diabetes. In vitro and animal experiments have extensively shown AGE-inhibiting and beneficial effects of in particular the specific AGE inhibitor aminoguanidine and AGE breaker alagebrium, and of the $B$ vitamins pyridoxamine and thiamine. The exact potential clinical value however, remains to be established. With the present review, we aimed to provide an overview of the current evidence from clinical studies on 1) clinical benefits of specific AGE inhibitors and AGE breakers and 2) potential AGE-inhibiting effects of therapies developed for purposes unrelated to the glycation pathway. We searched the PubMed Central database (using the search terms glycation or advanced glycation or advanced glycation endproduct in combination with therapy or treatment or inhibition and trial or clinical trial or randomized controlled trial or clinical study) and finally included 78 articles. We identified nine different categories of therapies with potential AGE-inhibiting capacities (i.e. specific AGE inhibitors and AGE breakers, B vitamins, aldose reductase inhibitors, glucose-, blood pressure- and lipid-lowering medication, medication against rheumatic diseases, weight-reduction therapies and AGE-restricted diets). Safety and/or efficacy in clinical studies with the specific AGE inhibitor aminoguanidine and AGE breaker alagebrium appeared to be of concern. The current evidence for AGE inhibition of therapies developed for purposes unrelated to glycation is fairly limited due to a large heterogeneity in study designs and/or measurement techniques, which have often been sub-optimal. More large, specific and well-designed studies are needed to fully elucidate their potential AGE-inhibiting effects in humans. 


\section{INTRODUCTION}

Diabetes is a common metabolic disease currently affecting around 346 million people worldwide, ${ }^{1}$ which is expected to rise to 552 million by $2030 .{ }^{2}$ Micro- and macrovascular diseases cause most of the excess morbidity and mortality in diabetes. Specifically, adults with diabetes experience a 2-fold increased risk for cardiovascular events ${ }^{3}$ and cardiovascular death ${ }^{4}$ relative to those without diabetes. Large studies in type 1 (T1DM) ${ }^{5}$ and type 2 diabetes (T2DM) ${ }^{6}$ have established that hyperglycaemia plays an important role in the pathogenesis of microvascular complications such as nephropathy, retinopathy and neuropathy and macrovascular complications such as atherosclerotic disease. Various mechanisms have been proposed to explain the increased vascular risk associated with hyperglycaemia; increased formation of advanced glycation endproducts (AGEs) by nonenzymatic glycation of proteins constitutes one such mechanism. ${ }^{7-9}$

Non-enzymatic glycation, first described by Louis Camille Maillard in the early 1900s (i.e. the Maillard reaction), involves the condensation reaction of the carbonyl group of sugar aldehydes with the $\mathrm{N}$-terminus or free amino groups of proteins via a nucleophilic addition, resulting first in the rapid formation of a Schiff base. Through acid-base catalysis, this labile adduct then undergoes rearrangements to the more stable Amadori-product. Only a small part of these relatively stable intermediate Amadori-products undergoes further oxidative reactions and can give rise to irreversible AGEs ${ }^{10}$ (Figure 2.1).

Because of their slow formation, it was long believed that AGEs accumulate only on long-lived extracellular proteins. However, a rapid extracellular AGE formation on shortlived proteins and intracellular AGE formation by reactive dicarbonyl compounds has attracted attention ${ }^{11-13}$ and is currently believed to be of even more importance than the 'traditional' Maillard reaction. These dicarbonyl compounds, such as methylglyoxal (MG), glyoxal, and 3-deoxyglucosone (3DG), can be formed not only from glucose-derived glycolytic intermediates, ${ }^{14}$ but also as a result of lipid peroxidation and by oxidative stress $^{15,16}$ (Figure 2.1).

It was not until 1980 that the pathophysiological significance of AGEs emerged in medical science, in particular in relation to ageing and in the aetiology of diabetic complications; ${ }^{17}$ these physiological consequences of the Maillard reaction have been described in excellent reviews. ${ }^{18-26}$ In brief, several mechanisms have been proposed by which AGEs could lead to diabetic complications: 1) accumulation of AGEs in the extracellular matrix causing aberrant cross-linking, resulting in a decrease of elasticity of vessels - arterial stiffness; ${ }^{27}$ 2) binding of AGEs to AGE-receptors [such as the receptor for advanced glycation end products (RAGE)] on different cell types and activation of key cell 


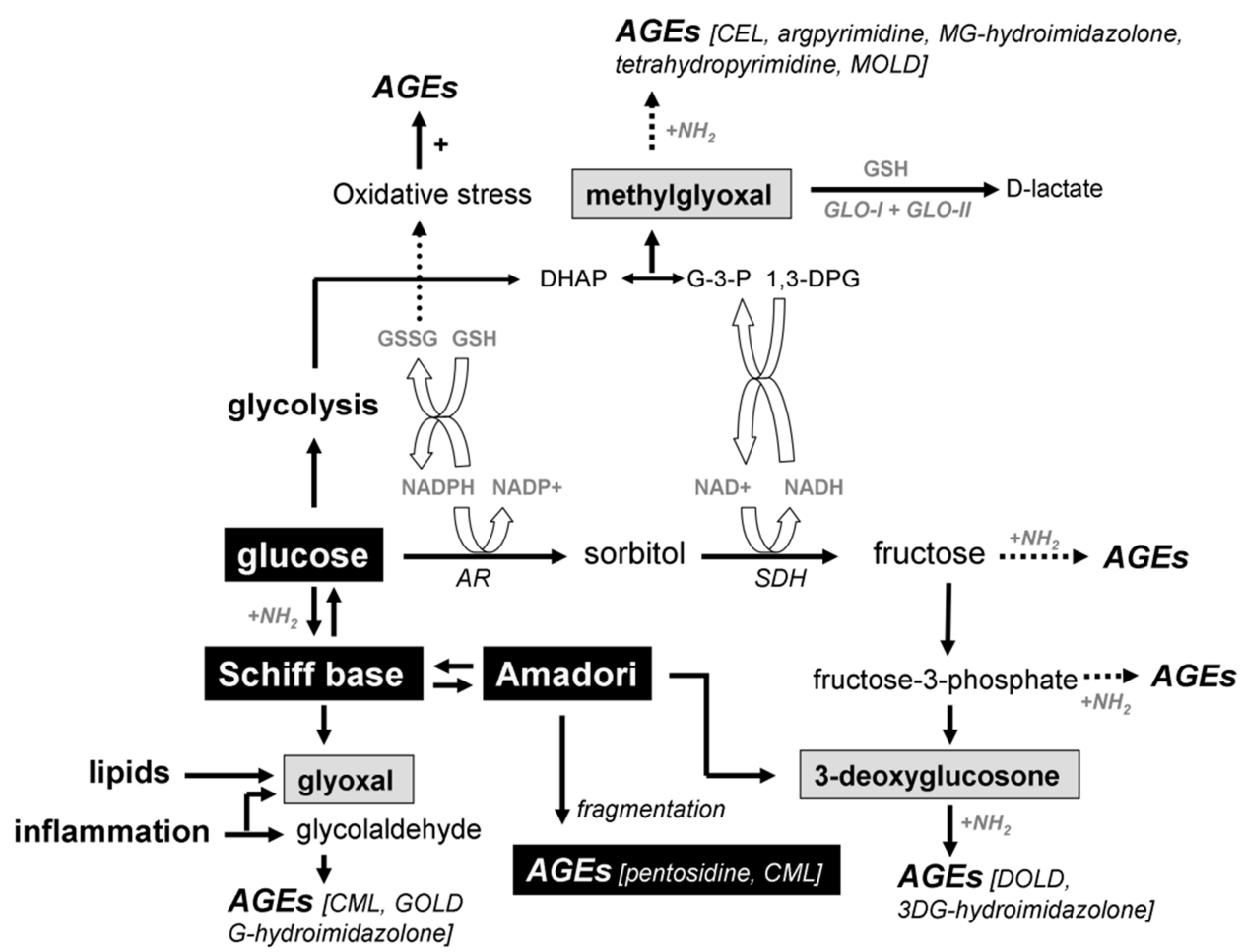

Figure 2.1 The formation of advanced glycation end products (AGEs). The classical pathway of the Maillard reaction is indicated in black boxes and the reactive dicarbonyl compounds pathway in grey boxes. An excess of glucose is also metabolized to sorbitol by aldose reductase (AR), accompanied by increased oxidation of ADPH to NADP+. A putative decreased level of NADPH, which is a cofactor for glutathione reductase, may lead to a depletion of reduced glutathione and thus to enhanced oxidative stress and the formation AGEs. Oxidation of sorbitol by sorbitol dehydrogenase (SDH) yields NADH and causes an increase in the ratio NADH/NAD+. An increase of the ratio NADH/NAD+ inhibits glyceraldehyde-3-phosphate dehydrogenase (GAPDH) and, subsequently, may lead to the accumulation of triose phosphates glyceraldehyde-3-phosphate (G-3-P) and dihydroxyacetone-phosphate (DHAP). Both trioses, which are also formed in the glycolysis, are precursors of methylglyoxal (MG). MG reacts with arginine residues to form MGhydroimidazolone, tetrahydropyrimidine and argpyrimidine and with lysine to form CEL and methyl-glyoxal-derived lysine dimer (MOLD). The sorbitol pathway may further increase the levels of MG by depletion of reduced glutathione (GSH) in the cell, which has a detoxification function in the glyoxalase pathway by facilitating the conversion of MG into D-lactate. Fructose and its derivative fructose-3-phosphate (F3P), are potent glycating agents; F3P is also hydrolysed to 3-deoxyglucosone (3DG), which reacts with protein amino groups to form the AGEs 3DG-hydroimidazolone and the 3DG-derived lysine dimer (DOLD). Glyoxal and glycolaldehyde are products of autoxidation of glucose or glucose adducts to proteins and may also be formed directly during oxidative degradation of polyunsaturated fatty acids and during myeloperoxidase-mediated degradation of serine at sites of inflammation. These compounds are able to modify the side chains of various amino acids in protein, including those of lysine and arginine, to form CML, glyoxal-hydroimidazolone, and the glyoxal-derived lysine dimer (GOLD). 
signalling pathways such as NF-KB activation with subsequent modulation of gene expression in vascular cells such as endothelial cells, smooth muscle cells and macrophages; ${ }^{28,29}$ and 3) intracellular AGE formation leading to quenching of nitric oxide and impaired function of growth factors. ${ }^{30}$

Given these deleterious effects of AGEs, agents with potential AGE-inhibitory properties, which thereby may retard the development of complications, are of interest. ${ }^{31}$ The burden of the glycation pathway may be decreased by therapies that intervene in the production of AGEs, i.e. by specifically scavenging AGE precursors (i.e. dicarbonyl compounds) or influencing mechanisms involved in AGE formation such as lipid peroxidation or oxidative stress, or by therapies that break vascular wall collagen crosslinks. Interestingly, a recent review pointed out that various AGE inhibitors and breakers may act primarily as chelators and that these agents work via the inhibition of metalcatalyzed oxidation reactions involved in AGE formation. ${ }^{32}$ In the last decade, numerous studies have been published about compounds with potential AGE-inhibitory effects, of which the results from in vitro and animal experimental work have been extensively reviewed. ${ }^{32-35}$ In particular, the AGE-inhibiting effects of the AGE inhibitor aminoguanidine and the AGE cross-link breaker alagebrium and of $B$ vitamins (pyridoxamine, thiamine and its synthetic derivative benfotiamine) have been extensively evaluated. ${ }^{36-65}$

Aminoguanidine, which is a small hydrazine-like molecule, inhibits the formation of AGEs by interaction with and quenching of dicarbonyl compounds. ${ }^{36}$ Numerous in vitro experiments and animal studies of experimental diabetes with aminoguanidine have shown prevention of diabetes-induced deteriorations of cardiovascular structure ${ }^{37,38}$ and function ${ }^{39}$ and of major long-term complications such as nephropathy, ${ }^{40-43}$ retinopathy ${ }^{44,45}$ and neuropathy. ${ }^{46,47}$ The cross-link breaker alagebrium (ALT-711) directly targets the biochemical pathway leading to stiffness of the cardiovascular system. ${ }^{51,52}$ In animal models, pharmacologic intervention with alagebrium has proven efficiency in reducing large artery stiffness, left ventricular mass and diastolic stiffness, ${ }^{48-52}$ atherosclerosis ${ }^{53}$ and diabetic nephropathy. ${ }^{54-56}$ Pyridoxamine ${ }^{57,58}$ and thiamine ${ }^{59}$ have also been found to be potent inhibitors of the formation of AGEs in vitro. In diabetic rats, pyridoxamine, thiamine and its synthetic derivative benfotiamine inhibited the formation of $A G E s^{60-64}$ and retarded the development of nephropathy ${ }^{60,63,65}$ and/or retinopathy. ${ }^{61,62}$

The potential clinical value of AGE-inhibiting interventions remains to be established, however. With the present review, we aimed to provide an overview of the current evidence from clinical studies on interventions in the glycation pathway on 1) the potential clinical benefits of specific AGE inhibitors and AGE breakers and 2) potential AGE-inhibiting effects of other drugs and/or therapies developed for purposes unrelated to glycation-based theories. 


\section{METHODS}

We searched the PubMed Central database (U.S. NIH free digital archive of biomedical and life sciences journal literature) for clinical studies on the effects of therapeutic interventions in relation to AGE inhibition. Search terms used included glycation or advanced glycation or advanced glycation endproduct in combination with therapy or treatment or inhibition and trial or clinical trial or randomized controlled trial or clinical study. We selected, based on the title and the abstract, articles that reported on the effects of interventions on circulating, urinary or tissue accumulation of AGEs and those evaluating specific AGE-inhibiting therapies, in a human setting. In addition, we searched reference lists of the retrieved articles to identify other eligible studies. At present, the potential AGE-inhibiting effects of the various therapies have mainly been assessed in either small exploratory and/or pilot studies or in post-hoc analyses of trials. We therefore decided to provide a complete overview rather than set strict limits (e.g. to study designs) as randomized controlled trials (RCTs) specifically designed to assess AGE-inhibiting effects are currently scarce or completely lacking for some therapies.

\section{RESULTS}

Our search (March 25, 2012) resulted in 398 potentially eligible articles. Of these, 313 were excluded because these reported in languages other than English ( $n=17)$, involved in vitro, ex vivo and/or cell experiments $(n=53)$ or animal studies $(n=45)$, were reviews $(n=84)$ or case reports $(n=1)$ and/or did not involve the evaluation of an intervention $(n=74)$ or AGEs as outcome parameters $(n=39)$. Three articles that reported no AGEs as outcome either, but involved specific AGE inhibition therapies, were accordingly included. In addition, the effects of different dialysis $(n=21)$ or mechanical ventilation $(n=4)$ modalities, islet transplantation $(n=1)$ or topical products $(n=1)$ were beyond the scope of this review. We thus found 58 eligible articles, added another 20 that we identified after searching the reference lists, and eventually included 78 articles. We identified nine different types of therapies with potential AGE-inhibiting capacities: 1) specific AGE inhibitors and AGE breakers; 2) B vitamins and synthetic derivatives of $B$ vitamins; 3 ) aldose reductase (AR) inhibitors; 4) glucose-lowering medication; 5) blood pressure-lowering medication; 6) lipid-lowering medication; 7) medication against rheumatic diseases; 8) weight-reduction therapies and 9) AGE-restricted diets. We additionally found eleven studies on the potential AGE-inhibiting effects of various 'other' therapies not specifically related to the 
above categories. For an overview of the characteristics and results of the included studies, please see Table 2.1 and 2.2 and Table A2.1-A2.8 in the Appendix.

\section{SPECIFIC AGE INHIBITORS AND AGE BREAKERS}

Table 2.1 summarizes clinical studies investigating the effects of aminoguanidine and the cross-link breaker alagebrium on diabetic complications, cardiovascular outcomes and/or measures of glycation.

The effects of aminoguanidine on diabetes-related complications and/or measures of glycation have been investigated in two large double-blind RCTs of individuals with T1DM $^{66}$ and T2DM. ${ }^{67}$ In individuals with T1DM, 2-4 years of aminoguanidine treatment did not result in a significant delay in time to doubling of serum creatinine (the primary endpoint). ${ }^{66}$ In individuals with T2DM, the trial that was planned to continue for two years to also investigate the effects of aminoguanidine on time to doubling of serum creatinine was terminated early due to an unfavourable perceived risk-to-benefit ratio. ${ }^{67}$

The effects of alagebrium on a range of cardiovascular outcome parameters have been tested in two double-blind RCTs of individuals with heart failure ${ }^{68}$ and hypertension. ${ }^{69}$ In individuals with heart failure, alagebrium showed no treatment effects after 36 weeks, $^{68}$ whereas in hypertensive individuals total arterial compliance, pulse wave velocity and pulse pressure were beneficially altered after 8 weeks of alagebrium treatment vs. placebo without apparent differences in mean arterial pressure, systemic resistance, cardiac output and heart rate. ${ }^{69}$ In addition, in two (small) uncontrolled (singlearm) studies in individuals with hypertension ${ }^{70}$ and diastolic heart failure, ${ }^{71}$ improvements in aortic augmentation index (as a marker of pulse wave reflection and arterial stiffness) and flow-mediated dilation (as a marker of endothelial function) were observed after eight weeks $^{70}$ and in left ventricular mass and diastolic function after sixteen weeks of alagebrium treatment ${ }^{71}$ with no changes in $\mathrm{VO}_{2} \max ^{71}$ left ventricular wall thickness, cardiac output, stroke volume, systolic and diastolic function, ${ }^{70}$ blood pressure, ${ }^{70,} 71$ brachial $^{70}$ and aortic $^{71}$ distensibility. 
Table 2.1 Potential clinical benefits of aminoguanidine and alagebrium

\begin{tabular}{|c|c|c|c|c|c|c|c|}
\hline Ref. & Therapy & $\begin{array}{l}\text { Patient } \\
\text { characteristics }\end{array}$ & Design & Treatment arms (n) & $\begin{array}{l}\text { Treatment } \\
\text { duration }\end{array}$ & Outcome measures & Results \\
\hline 66 & Aminoguanidine & $\begin{array}{l}\text { T1DM, } \\
\text { nephropathy, } \\
\text { retinopathy }\end{array}$ & $\begin{array}{l}\text { Double-blind } \\
\text { RCT }\end{array}$ & $\begin{array}{l}150 \mathrm{mg} \text { aminoguanidine } \\
\text { ( } n=229), 300 \mathrm{mg} \\
\text { aminoguanidine } \\
(\mathrm{n}=225), \text { placebo } \\
(\mathrm{n}=236)\end{array}$ & $2-4$ years & $\begin{array}{l}\text { Microvascular } \\
\text { complications }\end{array}$ & $\begin{array}{l}\text { Proteinuria and retinopathy } \\
\text { progression } \downarrow \text { in aminoguanidine vs. } \\
\text { placebo groups; no treatment } \\
\text { effects on doubling of Serum- } \\
\text { creatinine }\end{array}$ \\
\hline 67 & Aminoguanidine & $\begin{array}{l}\text { T2DM, renal } \\
\text { disease }\end{array}$ & $\begin{array}{l}\text { Double-blind } \\
\text { RCT }\end{array}$ & $\begin{array}{l}50-300 \mathrm{mg} \\
\text { aminoguanidine } \\
\text { ( } \mathrm{n}=207), 100-600 \mathrm{mg} \\
\text { aminoguanidine } \\
(\mathrm{n}=198), \text { placebo } \\
(\mathrm{n}=194)\end{array}$ & $\begin{array}{l}2 \text { years } \\
\text { planned }\end{array}$ & $\begin{array}{l}\text { Serum and urinary } \\
\text { AGEs, micro- and } \\
\text { macrovascular } \\
\text { complications }\end{array}$ & $\begin{array}{l}\text { Trial terminated due to } \\
\text { unfavourable perceived risk-to- } \\
\text { benefit ratio }\end{array}$ \\
\hline 68 & Alagebrium & $\begin{array}{l}\text { ND or DM, heart } \\
\text { failure }\end{array}$ & $\begin{array}{l}\text { Double-blind } \\
\text { RCT }\end{array}$ & $\begin{array}{l}400 \mathrm{mg} \text { alagebrium } \\
(n=50), \text { placebo }(n=52)\end{array}$ & 36 weeks & $\begin{array}{l}\text { Skin AF, VO2max, } \\
\text { systolic and diastolic } \\
\text { function }\end{array}$ & No treatment effects \\
\hline 69 & Alagebrium & $\begin{array}{l}\text { ND or stable } \\
\text { T2DM, } \\
\text { hypertension }\end{array}$ & $\begin{array}{l}\text { Double-blind } \\
\text { RCT }\end{array}$ & $\begin{array}{l}210 \text { mg alagebrium } \\
(n=62), \text { placebo }(n=31)\end{array}$ & 8 weeks & $\begin{array}{l}\text { 'Cardiac and arterial } \\
\text { properties', BP }\end{array}$ & $\begin{array}{l}\text { PP, aortic compliance, PWV, SV/PP } \\
\text { improved in alagebrium vs. placebo } \\
\text { group; no treatment effects on SBP, } \\
\text { cardiac output, systemic vascular } \\
\text { resistance, heart rate, SV and aortic } \\
\text { root diameter }\end{array}$ \\
\hline 70 & Alagebrium & $\begin{array}{l}\text { Systolic } \\
\text { hypertension }\end{array}$ & $\begin{array}{l}\text { Uncontrolled } \\
\text { (single-arm) } \\
\text { study }\end{array}$ & $\begin{array}{l}420 \mathrm{mg} \text { alagebrium } \\
(\mathrm{n}=13), \text { no control group }\end{array}$ & 8 weeks & $\begin{array}{l}\text { 'Cardiac and arterial } \\
\text { properties', BP }\end{array}$ & $\begin{array}{l}\text { Carotid AI, FMD improved after } \\
\text { alagebrium treatment; no changes } \\
\text { in BP, brachial distensibility, LV wall } \\
\text { thickness, cardiac output, SV, } \\
\text { systolic and diastolic function }\end{array}$ \\
\hline 71 & Alagebrium & $\begin{array}{l}\text { ND or DM, } \\
\text { diastolic heart } \\
\text { failure }\end{array}$ & $\begin{array}{l}\text { Uncontrolled } \\
\text { (single-arm) } \\
\text { open-label } \\
\text { study }\end{array}$ & $\begin{array}{l}420 \mathrm{mg} \text { alagebrium } \\
(\mathrm{n}=23), \text { no control group }\end{array}$ & 16 weeks & $\begin{array}{l}\text { BP, VO2max, } \\
\text { diastolic function, } \\
\text { LV mass, aortic } \\
\text { distensibility }\end{array}$ & $\begin{array}{l}\text { LV mass and diastolic function } \\
\text { improved after alagebrium } \\
\text { treatment; no changes in BP, VO2 } \\
\text { max, aortic distensibility }\end{array}$ \\
\hline
\end{tabular}




\section{B VITAMINS AND SYNTHETIC DERIVATIVES OF B VITAMINS}

Table 2.2 summarizes the potential AGE-inhibiting effects of vitamin B6 (pyridoxamine and pyridoxine), vitamin B1 (thiamine) and a synthetic derivative of vitamin B1 (benfotiamine).

A phase-II RCT in individuals with T1DM or T2DM showed decreased plasma $\mathrm{N}^{\varepsilon}(1-$ carboxymethyl)lysine (CML) and $\mathrm{N}^{\varepsilon}$ (1-carboxyethyl)lysine CEL after 24 weeks of pyridoxamine treatment vs. placebo. ${ }^{72}$ In contrast, in patients on haemodialysis and after eight weeks of treatment, pyridoxine combined with thiamine treatment showed no effects on plasma AGEs and pentosidine. ${ }^{73}$

Benfotiamine treatment for 12-18 weeks showed no treatment effects vs. placebo on plasma AGEs in haemodialysis patients. ${ }^{74}$ A 3-day benfotiamine treatment in individuals with T2DM did not result in differences in serum CML and MG derivatives before and two, four and six hours after a high AGE containing meal compared with a control group. However, the increase in serum CML and MG derivatives 4-hour post meal as seen in the control group was blunted in the benfotiamine-treated group. ${ }^{75}$ In addition, an uncontrolled (single-arm) study on benfotiamine plus alpha-lipoic acid in T1DM showed decreased angiopoietin levels (as a marker of increased intracellular methylglyoxal adducts in endothelial cells ${ }^{76}$ ) after treatment. ${ }^{77}$

\section{ALDOSE REDUCTASE INHIBITORS}

No (placebo-controlled) RCTs of the potential AGE-inhibiting effects of AR inhibitors were found. However, a few (small) other clinical studies on the AR inhibitor epalrestat also investigated the effects on AGEs, which are summarized in Table A2.1 - Appendix. An open-label nonrandomized controlled trial in individuals with T2DM showed a decrease in serum CML after 1 year of epalrestat treatment vs. the control group without epalrestat treatment, ${ }^{78}$ whereas a small uncontrolled (single-arm) study showed no such effects on plasma CML after 6 months of treatment. ${ }^{79}$ In two additional articles, both on (small) observational and uncontrolled (single-arm) studies, lower erythrocyte levels of $\mathrm{CML}$, $3 D G,{ }^{80}$ sorbitol 3-phosphate and fructose 3-phosphate ${ }^{81}$ were reported in individuals with T2DM who had been treated with epalrestat for at least one ${ }^{81}$ or two ${ }^{80}$ months (vs. those who were not) and/or after $1^{81}$ or 2 months ${ }^{80}$ of epalrestat treatment. 
Table 2.2 Potential AGE-inhibiting effects of vitamin B and synthetic derivatives of vitamin B

\begin{tabular}{|c|c|c|c|c|c|c|c|}
\hline Ref. & Therapy & $\begin{array}{l}\text { Patient } \\
\text { characteristics }\end{array}$ & Design & Treatment arms $(n)$ & $\begin{array}{l}\text { Treatment } \\
\text { duration }\end{array}$ & $\begin{array}{l}\text { Glycation-related } \\
\text { outcome }\end{array}$ & Results \\
\hline 72 & Pyridoxamine & $\begin{array}{l}\text { T1DM or T2DM, } \\
\text { nephropathy }\end{array}$ & $\begin{array}{l}\text { Double-blind RCT } \\
\text { (Phase II) }\end{array}$ & $\begin{array}{l}100 \mathrm{mg} \text { pyridoxamine } \\
(n=23), \text { placebo }(n=10)\end{array}$ & 24 weeks & $\begin{array}{l}\text { Plasma-CML and } \\
\text { CEL (GC-MS) }\end{array}$ & $\begin{array}{l}\text { Plasma-CEL (n.s.) and CML } \downarrow \text { in } \\
\text { pyridoxamine vs. placebo } \\
\text { group }\end{array}$ \\
\hline${ }^{73} *$ & $\begin{array}{l}\text { Pyridoxine + } \\
\text { thiamine }\end{array}$ & HD patients & Double-blind RCT & $\begin{array}{l}250 \text { mg thiamine }+200 \\
\text { mg pyridoxine }(n=25) \text {, } \\
\text { placebo }(n=25)\end{array}$ & 8 weeks & $\begin{array}{l}\text { Plasma-AGEs and } \\
\text { pentosidine }\end{array}$ & No treatment effects \\
\hline 74 & Benfotiamine & HD patients & $\begin{array}{l}\text { 1: uncontrolled } \\
\text { (single-arm) study; } \\
\text { 2: single-blind RCT }\end{array}$ & $\begin{array}{l}\text { 1: } 300-450 \mathrm{mg} \\
\text { benfotiamine ( } n=15), \text { no } \\
\text { control group; } 2: 600 \mathrm{mg} \\
\text { benfotiamine }(n=15), \\
\text { placebo }(n=8)\end{array}$ & $\begin{array}{l}1 \text { and } 2: 12- \\
18 \text { weeks }\end{array}$ & $\begin{array}{l}1 \text { and 2: Plasma- } \\
\text { AGEs } \\
\text { (fluorescence) }\end{array}$ & 1 and 2: no treatment effects \\
\hline 75 & Benfotiamine & T2DM & $\begin{array}{l}\text { Open-label } \\
\text { nonrandomised } \\
\text { crossover study }\end{array}$ & $\begin{array}{l}1050 \text { mg benfotiamine } \\
(n=13) ; \text { control group } \\
(n=13), \text { crossover design }\end{array}$ & 3 days & $\begin{array}{l}\text { Serum-CML and } \\
\text { MG derivatives } \\
\text { (ELISA) }\end{array}$ & $\begin{array}{l}\text { No difference between groups } \\
\text { in Serum-CML and MG } \\
\text { derivatives; increase in Serum- } \\
\mathrm{CML} \text { and } \mathrm{MG} 4 \mathrm{~h} \text { post high-AGE } \\
\text { meal blunted in benfotiamine } \\
\text { group }\end{array}$ \\
\hline 77 & $\begin{array}{l}\text { Benfotiamine }+\alpha- \\
\text { lipoic acid }\end{array}$ & T1DM & $\begin{array}{l}\text { Uncontrolled } \\
\text { (single-arm) study }\end{array}$ & $\begin{array}{l}600 \mathrm{mg} \text { benfotiamine }+ \\
1200 \mathrm{mg} \alpha \text {-lipoic acid } \\
(n=9), \text { no control group }\end{array}$ & 28 days & Angiopoietin-2 & $\begin{array}{l}\text { Angiopoietin- } 2 \downarrow \text { after } \\
\text { benfotiamine }+\alpha \text {-lipoic acid } \\
\text { treatment }\end{array}$ \\
\hline
\end{tabular}

GC-MS, Gas chromatography-mass spectrometry; $H D$, haemodialysis; $R C T$, randomized controlled trial; T1DM, type 1 diabetes; T2DM, type 2 diabetes.

*Results based on abstract only. 


\section{GLUCOSE-LOWERING MEDICATION}

Tabel A2.2 - Appendix summarizes potential AGE-inhibiting effects of specific glucoselowering medication (i.e. metformin, rosiglitazone, sulfonylureas, acarbose) or improvement of glycaemic control with intensified anti-hyperglycaemic treatment in general.

Three observational studies showed, in individuals with T2DM who were on metformin treatment vs. those who were not, ${ }^{82-84}$ lower serum fructosamine, ${ }^{84}$ lower plasma MG, but 3DG ${ }^{82}$ and lower LDL apoB100 levels of glyoxal-hydroimidazolone (G-H1), MG-H1, 3DG-H and $N^{\omega}$-(carboxymethyl)arginine (CMA), but not CML, CEL, MG-derived lysine dimer (MOLD) and pentosidine. ${ }^{83}$ When metformin treatment was compared with other anti-hyperglycaemic treatment (i.e. repaglinide ${ }^{85,86}$ or pioglitazone ${ }^{87}$ ) in two randomized trials in T2DM, similar 'lowering effects' of metformin and these other glucose-lowering treatments (i.e. no between-treatment effects) were found on blood levels of $3 D^{85}$ and serum pentosidine, ${ }^{87}$ with no treatment effects on plasma CML, CEL, pyrraline and AGE peptides. ${ }^{85,86}$

Several articles described the effects of intensified anti-hyperglycaemic treatment (mostly with insulin) on measures of glycation. In a small sub-sample of the DCCT trial, no treatment effects on skin fructosyllysine (FL), CML and pentosidine were found, ${ }^{88}$ whereas in a larger sub-sample of the same trial skin collagen glycation and skin CML, pentosidine and fluorescence decreased in the intensive vs. the conventional anti-hyperglycaemic treatment group. ${ }^{89}$ No between-treatment effects were found when insulin was compared with other anti-hyperglycaemic therapies in an additional RCT. ${ }^{90}$ Out of the six uncontrolled (single-arm) studies, four studies showed AGE-inhibiting effects (i.e. on Amadori-modified plasma proteins, ${ }^{91}$ serum fructosamine ${ }^{91,}{ }^{92}$ and CML, pentosidine, ${ }^{93,94}$ AGEs $^{95}$ and skin collagen glycation ${ }^{92}$ ) after intensified anti-hyperglycaemic treatment. The remaining two found decreased skin $\mathrm{FL}$, but not $\mathrm{CML}, N^{\varepsilon}$ (carboxymethyl)hydroxylysine (CMhL), pentosidine and fluorescence, ${ }^{96}$ or no (significant) effects on serum CML and AGEs and increased MG- $\mathrm{H}^{97}$ after intensified anti-hyperglycaemic treatment.

\section{BLOOD PRESSURE-LOWERING MEDICATION}

Table A2.3 - Appendix summarizes clinical studies on the potential AGE-inhibiting effects of angiotensin receptor blockers (ARBs), angiotensin converting enzyme (ACE) inhibitors and calcium channel blockers.

The effects of the ARB, irbesartan, on measures of glycation have been investigated in post-hoc analyses of two double-blind placebo-controlled RCTs of individuals with 
T2DM, hypertension and nephropathy. ${ }^{98-100}$ Both RCTs showed no treatment effects of 2 years of irbesartan treatment (300 mg) on plasma levels of $\mathrm{CML}^{98,99}$ pentosidine, ${ }^{98} \mathrm{CEL}^{99}$ and AGE peptides. ${ }^{100}$ Similarly, an open-label RCT in individuals with hypertension and cardiac diastolic dysfunction showed no effects of 6 months of treatment with eprosartan on top of other blood pressure-lowering medication on skin autofluorescence (as a marker of tissue AGE accumulation) or plasma levels of CML, CEL and pentosidine. ${ }^{101}$ In contrast however, a (small) open-label RCT in haemodialysis patients showed a decrease in plasma AGEs in the group receiving losartan for 12 months vs. those on other blood pressurelowering medication. ${ }^{102}$ In addition, several uncontrolled (single-arm) studies showed decreases in plasma, serum and/or urinary AGEs after treatment with candesartan ${ }^{103}$ and valsartan. ${ }^{104-106}$ In contrast, an additional uncontrolled (single-arm) study that investigated the influence of 2 months of irbesartan treatment on a range of glycation measures, showed increased plasma levels of FL, CML, CEL and pentosidine after irbesartan treatment, but also decreased plasma 3DG-H and urinary MG-H1 and G-H1 with no treatment effects on plasma MG-H1, G-H1, G-derived lysine dimer (GOLD) and MOLD and urinary FL, CML, CEL, 3DG-H, GOLD, MOLD and pentosidine. ${ }^{107}$

One (small) open-label nonrandomized controlled trial showed that plasma AGEs (as determined by fluorescence), but not plasma CML, were decreased after two months of treatment with the ACE inhibitor, ramipril, vs. other blood pressure-lowering treatment in non-diabetic patients with chronic kidney disease. ${ }^{108}$

An article on a double-blind placebo-controlled RCT on the calcium channel blocker, amlodipine, reported no effects on plasma CML and pentosidine. ${ }^{98}$ Another open-label RCT that compared azelnidipine with amlodipine reported decreased serum AGEs in the azelnidipine vs. the amlodipine group after six months of treatment. ${ }^{109}$

\section{LIPID-LOWERING MEDICATION}

Table A2.4 - Appendix summarizes the potential AGE-inhibiting effects of several types of statin treatment (i.e. atorvastatin, cerivastatin and simvastatin).

A double-blind RCT in individuals with impaired glucose metabolism or T2DM showed decreased serum CML after 12 -week cerivastatin treatment vs. placebo, ${ }^{110}$ whereas in another double-blind RCT in individuals with T2DM, no treatment effects of atorvastatin (for six months) were found on serum AGEs. ${ }^{111}$ In an open-label RCT in patients with T2DM and carotid artery stenosis who were - four months prior to endarterectomy randomized to simvastatin plus a 'healthy eating diet' [formerly referred to as American Heart Association (AHA) step 1 diet] or diet only, decreased plaque expression of AGEs and RAGE was found in the simvastatin plus diet group compared with the diet only group. ${ }^{112}$ Two additional studies (i.e. an open-label non-randomized controlled trial in $\mathrm{T}^{2} \mathrm{DM}^{113}$ and 
an uncontrolled (single-arm) study in non-alcoholic steatohepatitis ${ }^{114}$ ) showed decreased serum AGEs after atorvastatin treatment.

\section{MEDICATION AGAINST RHEUMATIC DISEASES}

No (placebo-controlled) RCTs on the potential AGE-inhibiting effects of medication against rheumatoid arthritis were found; the effects on measures of glycation from several other clinical studies on anti-rheumatic treatment are summarized in Table A2.5 - Appendix. Two uncontrolled studies in individuals with rheumatoid arthritis showed decreased serum and urinary pentosidine levels after anti-TNF treatment with infliximab for 54 weeks ${ }^{115}$ or etarnecept for six months. ${ }^{116}$ A similar study on the effects of methothrexate also showed decreased serum and urinary pentosidine levels after six months of treatment. $^{117}$

\section{WEIGHT-REDUCTION THERAPY}

Table A2.6 - Appendix summarizes studies on potential AGE-inhibiting effects of weightreduction therapies [i.e. with orlistat, energy-restricted diets and/or exercise].

Uncontrolled $^{118-120}$ and open-label nonrandomized controlled ${ }^{121}$ studies investigating the effects of energy-restricted diets (alone $\mathrm{e}^{119,120}$ or in combination with orlistat treatment $^{118}$ ) or an exercise program ${ }^{121}$ showed decreased levels of serum AGEs, ${ }^{118,}{ }^{119}$ urinary pentosidine ${ }^{120}$ and serum $\mathrm{CML}$, but not serum pentosidine, ${ }^{121}$ after treatment. In addition, two studies on the short-term effects of orlistat showed that high-AGE-meal induced acute increases in serum AGEs were blunted when orlistat was administered immediately post-meal in women with polycystic ovary syndrome ${ }^{122}$ and healthy controls $^{122,123}$ but not in women with T2DM. ${ }^{123}$

\section{AGE-RESTRICTED DIETS}

AGE-restricted diets (i.e. advice to boil, poach, stew or steam food and to avoid frying, baking or grilling methods) were found to decrease serum CML and MG derivatives when compared to standard (isocaloric) diets in four studies in individuals with T1DM, ${ }^{124}$ $\mathrm{T}^{2} \mathrm{DM}^{124,}{ }^{125}$ or on peritoneal dialysis ${ }^{126}$ and in healthy individuals. ${ }^{127}$ In addition, AGE receptor 1 (AGER1) and RAGE expression of peripheral blood mononuclear cells was decreased after such an AGE-restricted diet in healthy individuals, ${ }^{127}$ but increased in those with T2DM ${ }^{125}$ (Table A2.7 - Appendix). 


\section{0. 'OTHER' THERAPIES}

Several other therapies have been described in relation to potential AGE inhibition (summarized in Table A2.8 - Appendix).

A placebo-controlled RCT of 6 months of oestrogen treatment showed decreased pentosidine (although non-significantly so) and non-fluorescent compound 1 levels in vaginal epithelium of postmenopausal women with stress urinary incontinence. ${ }^{128}$ Iron supplementation decreased serum fructosamine levels in anaemia patients, ${ }^{129}$ whereas no such effects and no differences between iron supplementation only or in combination with anti-H pylori treatment were found in anaemic $H$ pylori patients. ${ }^{130}$ In individuals with T2DM, treatment with dehydroepiandrosterone, ${ }^{131}$ aspirin ${ }^{132}$ and Danggui Sini Decoction (a compound in traditional Chinese herbal medicine) ${ }^{133}$ decreased plasma pentosidine, skin-tissue pentosidine and plasma AGEs, respectively. In addition, in haemodialysis patients, 1 year of sevelamer (i.e. phosphate binder) treatment on top of calcium carbonate decreased plasma pentosidine vs. calcium carbonate treatment only. ${ }^{134}$

Three RCTs of vitamin E vs. no treatment reported no effects on serum fructosamine, ${ }^{135}$ plasma pentosidine ${ }^{136}$ and other markers of protein and LDL glycation. ${ }^{137}$ In addition, no treatment effects on serum AGEs were found of anti-oxidant treatment with propionyl L-carnitine, sildenafil or both in individuals with T2DM and erectile dysfunction. $^{138}$

\section{DISCUSSION}

The present review evaluated the current literature of clinical studies on potential AGEinhibiting effects of different therapies, either specifically developed for AGE inhibition or developed for interventions unrelated to glycation-based theories.

Large trials on aminoguanidine showed no significant reduction in the primary endpoint (i.e. time to doubling of serum creatinine) ${ }^{66}$ or were terminated early due to an unfavourable perceived risk-to-benefit ratio. ${ }^{67}$ Due to safety concerns resulting from its additional effects (e.g. pro-oxidant activities ${ }^{139}$ and inhibition of NO synthase ${ }^{140}$ ), it is currently unlikely that aminoguanidine will be used in the treatment of diabetic complications. ${ }^{141}$ One RCT on alagebrium showed beneficial effects on a range of cardiovascular variables in hypertensive individuals, ${ }^{69}$ whereas another in heart failure patients failed to do so. ${ }^{68}$ Besides those reported on in the current review, several additional clinical trials on alagebrium have been terminated early (due to financial constraints) and/or have not yet been published (information available via clinicaltrials.gov ${ }^{142}$ ). Although the results from experimental studies concerning 
aminoguanidine ${ }^{36-47}$ and alagebrium ${ }^{48-56}$ were promising, it seems that safety and/or efficacy in clinical studies are of concern.

The natural vitamin B6 metabolite, pyridoxamine, has been shown to be a potent inhibitor of the formation of AGEs in in vitro ${ }^{57,58,143}$ and animal experiments. ${ }^{60,62}$ This effect of pyridoxamine is most probably due to blocking of the oxidative degradation of the glucose-derived Amadori intermediate or due to the quenching of dicarbonyl compounds. $^{32,143}$ Pyridoxamine treatment decreased not only plasma levels of CML and CEL, but also reduced the change from baseline creatinine in individuals with T1DM or T2DM. $^{72}$ However, another placebo-controlled RCT showed no effects on changes in creatinine. ${ }^{144}$ Furthermore, pyridoxine treatment combined with thiamine did not show an effect on plasma AGEs and pentosidine. ${ }^{73}$ Thus, the clinical evidence on potential AGEinhibiting effects of these B6 vitamers is still limited.

Thiamine and benfotiamine have also been found to inhibit the formation of AGEs in in vitro ${ }^{59}$ and animal experiments. ${ }^{60-64}$ These effects of thiamine and benfotiamine are most probably due to maximizing transketolase activity, which stimulates the anaerobic pentose pathway of glycolysis thereby reducing glycolytic metabolites involved in the formation of AGEs. ${ }^{61}$ Regardless of the limited clinical evidence of AGE-inhibiting effects of these vitamin B1 metabolites, ${ }^{73-75,77}$ two trials on thiamine supplementation have shown decreased urinary albumin excretion, ${ }^{145,}{ }^{146}$ although benfotiamine supplementation did not result in such effects. ${ }^{147}$ Whether beneficial effects of thiamine and/or benfotiamine treatment on vascular complications in individuals with diabetes, if any, are mediated through their inhibitory effects on the glycation pathway, remains to be established.

Given the similarities in structure between metformin and aminoguanidine, it has been proposed that metformin detoxifies dicarbonyls in a similar way as aminoguanidine, thereby preventing the subsequent production of AGEs. ${ }^{148}$ This effect of metformin might explain the beneficial impact of metformin on cardiovascular complications of T2DM in the UKPDS study. ${ }^{149}$ A number of studies, which should be interpreted with care due to their uncontrolled and/or observational (i.e. cross-sectional) designs, showed beneficial 'effects' of metformin on a range of measures of glycation. ${ }^{82-84,150}$ However, in two randomized trials no additional effects of metformin were observed in comparison to other antihyperglycaemic treatments, ${ }^{85-87}$ which suggests that these AGE-inhibiting effects result from an improvement in glycaemic control rather than from a specific dicarbonyl detoxification.

Blockade of the RAAS system has been shown to reduce progression of nephropathy and other cardiovascular outcomes in patients with T2DM. ${ }^{151,152}$ In vitro ${ }^{153}$ and animal models ${ }^{154-161}$ showed inhibiting effects of ARBs and ACE inhibitors on AGEs and their sequelae via their anti-oxidant and metal chelating properties. ${ }^{32,157,162}$ These data led to the hypothesis that such AGE-inhibiting effects could explain, at least in part, the beneficial effects of ARBs and ACE inhibitors in patients with T2DM. However, although 
several small (and mostly uncontrolled) clinical studies have also shown decreases in circulating ${ }^{102,104-107}$ or urinary ${ }^{103,105,107}$ AGEs after treatment with ARBs, two large RCTs on irbesartan failed to show this. ${ }^{98-100}$ Altogether, these findings suggest that AGE-inhibiting effects of ARBs may be restricted to experimental models of diabetes. With only one (small) nonrandomized clinical study on ramipril so $\mathrm{far}^{108}$ the clinical evidence for potential AGE-inhibiting effects of ACE inhibitors is currently limited.

Besides its lipid-lowering potential, statin treatment may exert beneficial effects through its anti-oxidant properties, ${ }^{163-165}$ thereby potentially decreasing lipid peroxidation ${ }^{163}$ and the (subsequent) production of AGEs. ${ }^{164}$ Indeed, several (small) clinical studies with statin have shown decreased levels of AGEs in serum ${ }^{113,114}$ as well as in plaques. ${ }^{112}$ However, placebo-controlled RCTs on statins so far showed either reductions in serum $\mathrm{CML}^{110}$ or no effects on serum AGEs. ${ }^{111}$ More and larger studies, preferably RCTs, are needed to fully clarify the potentials of statin treatment for AGE inhibition.

All ten (mostly small) included studies on weight-reduction therapies and/or AGErestricted diets showed decreases in circulating AGEs. ${ }^{118-123}$ Whether the decreases in serum AGEs, as seen after AGE-restricted diets, are due to the decreased consumption of AGEs or rather a reflection of the beneficial effects of a healthier diet in general (e.g. an improvement in glycaemic/metabolic control), remains to be established. In addition, whether the beneficial effects on the glycation pathway may contribute to the decreased cardiovascular risk associated with these lifestyle interventions requires further investigation.

Although glycation-related outcome measures used differed between studies, effects on circulating levels of AGEs have most often been assessed. It should be emphasised, however, that it is uncertain whether elevated circulating AGE levels adequately reflect the burden of the glycation pathway in the organs/tissues. Importantly, we therefore cannot fully discard the possibility that the discrepancy between the results from clinical studies, as summarized in this review, and the beneficial effects of various therapies previously shown in in vitro and animal experiments lies in the type of tissue examined. It remains possible that the potential beneficial effects of the different therapies on accumulation or burden of AGEs in organs/tissues may not translate to changes in plasma levels. To further clarify the exact clinical impact, future research is needed and should include more and different measurements of advanced glycation (e.g. free methylglyoxal, intracellular AGE concentrations and/or tissue AGE accumulation), which may better reflect the glycation processes that may be affected by treatment.

In addition, the quantitative determination of specific AGE epitopes by using specific analytical techniques such as high-performance liquid chromatography ${ }^{166}$ and/or (ultra performance) liquid chromatography tandem mass spectrometry ${ }^{167,168}$ is, at present, considered to be most accurate. ${ }^{166}$ However, in the intervention studies currently reviewed, immunoassays have most often been used (for details see Table 2.2 and A2.1- 
A2.8 in the Appendix). These studies in which AGEs have been determined by immunoassays should be interpreted with caution as these only yield semi-quantitative results. Moreover, the differences in techniques used between studies may in part explain the contrasting results as reported in the present review.

Comparing (or pooling) the results (or effect sizes) of the different studies, even within a single type or group of therapies, is particularly difficult or impossible due to the large heterogeneity not only in the outcome measure evaluated and techniques used (as discussed above), but also in the study designs and subjects' characteristics. In addition, a wide variety of compounds has been described in relation to (potential) AGE-inhibiting effects, which all are thought to work via different mechanisms. Future studies should determine which (glycation-related) outcome measures reflect the glycation pathway most accurately and are most suitable for intervention studies to improve possibilities for comparing and/or pooling results and evaluating the potentials for different therapies to inhibit the glycation pathway.

\section{CONCLUDING REMARKS}

It is now well recognized that the accumulation of AGEs in tissues has an important role in the pathogenesis of diabetic complications. Therefore, agents with AGE-inhibitory properties, which thereby may retard the development of complications, are of interest. Although experimental studies concerning the specific AGE inhibitor aminoguanidine and AGE breaker alagebrium clearly resulted in beneficial effects, it seems that safety and/or efficacy in clinical studies with these compounds are of concern. With regard to the other therapies developed for purposes unrelated to glycation and summarized herein, the current evidence is fairly limited. More large, specific and well-designed studies are needed to fully elucidate their potential AGE-inhibiting effects. In addition, future studies should determine which (glycation-related) outcome measures reflect the glycation pathway most accurately and are most suitable for intervention studies to improve possibilities of comparing and/or pooling results and evaluating the potentials for different therapies to inhibit the glycation pathway. 


\section{REFERENCES}

1. World Health Organization. Diabetes: Fact sheet no. 312. 2011;Accessed February 2012

2. International Diabetes Federation. Idf diabetes atlas: The global burden, 5th edition [online]. 2011;Accessed February 2012

3. The Emerging Risk Factors Collaboration, Sarwar N, Gao P, Seshasai SR, Gobin R, Kaptoge S, et al. Diabetes mellitus, fasting blood glucose concentration, and risk of vascular disease: A collaborative meta-analysis of 102 prospective studies. Lancet. 2010;375:2215-2222

4. The Emerging Risk Factors Collaboration, Seshasai SR, Kaptoge S, Thompson A, Di Angelantonio E, Gao P, et al. Diabetes mellitus, fasting glucose, and risk of cause-specific death. N Engl J Med. 2011;364:829-841

5. The Diabetes Control and Complications Trial Research Group. The effect of intensive treatment of diabetes on the development and progression of long-term complications in insulin-dependent diabetes mellitus. $N$ Engl J Med. 1993;329:977-986

6. UK Prospective Diabetes Study (UKPDS) Group. Intensive blood-glucose control with sulphonylureas or insulin compared with conventional treatment and risk of complications in patients with type 2 diabetes (ukpds 33) Lancet. 1998;352:837-853

7. Vlassara H, Palace MR. Diabetes and advanced glycation endproducts. J Intern Med. 2002;251:87-101

8. Brownlee M. Biochemistry and molecular cell biology of diabetic complications. Nature. 2001;414:813-820

9. Stitt AW. Advanced glycation: An important pathological event in diabetic and age related ocular disease. $\mathrm{Br}$ J Ophthalmol. 2001;85:746-753

10. Monnier VM, Cerami A. Nonenzymatic browning in vivo: Possible process for aging of long-lived proteins. Science. 1981;211:491-493

11. Westwood ME, Thornalley PJ. Molecular characteristics of methylglyoxal-modified bovine and human serum albumins. Comparison with glucose-derived advanced glycation endproduct-modified serum albumins. J Protein Chem. 1995;14:359-372

12. Giardino I, Edelstein D, Brownlee M. Nonenzymatic glycosylation in vitro and in bovine endothelial cells alters basic fibroblast growth factor activity. A model for intracellular glycosylation in diabetes. J Clin Invest. 1994;94:110-117

13. Giardino I, Edelstein D, Brownlee M. Bcl-2 expression or antioxidants prevent hyperglycemia-induced formation of intracellular advanced glycation endproducts in bovine endothelial cells. J Clin Invest. 1996;97:1422-1428

14. Thornalley PJ, Langborg A, Minhas HS. Formation of glyoxal, methylglyoxal and 3-deoxyglucosone in the glycation of proteins by glucose. Biochem J. 1999;344 Pt 1:109-116

15. Fu MX, Requena JR, Jenkins AJ, Lyons TJ, Baynes JW, Thorpe SR. The advanced glycation end product, nepsilon-(carboxymethyl)lysine, is a product of both lipid peroxidation and glycoxidation reactions. $J$ Biol Chem. 1996;271:9982-9986

16. Negre-Salvayre A, Coatrieux C, Ingueneau C, Salvayre R. Advanced lipid peroxidation end products in oxidative damage to proteins. Potential role in diseases and therapeutic prospects for the inhibitors. $\mathrm{Br} J$ Pharmacol. 2008;153:6-20

17. Monnier VM, Stevens VJ, Cerami A. Maillard reactions involving proteins and carbohydrates in vivo: Relevance to diabetes mellitus and aging. Prog Food Nutr Sci. 1981;5:315-327

18. Ahmed N. Advanced glycation endproducts--role in pathology of diabetic complications. Diabetes Res Clin Pract. 2005;67:3-21

19. Baynes JW. The role of ages in aging: Causation or correlation. Exp Gerontol. 2001;36:1527-1537

20. Singh R, Barden A, Mori T, Beilin L. Advanced glycation end-products: A review. Diabetologia. 2001;44:129146

21. Smit AJ, Lutgers HL. The clinical relevance of advanced glycation endproducts (age) and recent developments in pharmaceutics to reduce age accumulation. Curr Med Chem. 2004;11:2767-2784

22. Thornalley PJ. Glycation in diabetic neuropathy: Characteristics, consequences, causes, and therapeutic options. Int Rev Neurobiol. 2002;50:37-57

23. Tilton RG. Diabetic vascular dysfunction: Links to glucose-induced reductive stress and vegf. Microsc Res Tech. 2002;57:390-407 
24. Goh SY, Cooper ME. The role of advanced glycation end products in progression and complications of diabetes. J Clin Endocrinol Metab. 2008;93:1143-1152

25. Ahmed N, Thornalley PJ. Advanced glycation endproducts: What is their relevance to diabetic complications? Diabetes Obes Metab. 2007;9:233-245

26. Goldin A, Beckman JA, Schmidt AM, Creager MA. Advanced glycation end products: Sparking the development of diabetic vascular injury. Circulation. 2006;114:597-605

27. Aronson D. Cross-linking of glycated collagen in the pathogenesis of arterial and myocardial stiffening of aging and diabetes. J Hypertens. 2003;21:3-12

28. Miyazaki A, Nakayama $\mathrm{H}$, Horiuchi S. Scavenger receptors that recognize advanced glycation end products. Trends Cardiovasc Med. 2002;12:258-262

29. Stern DM, Yan SD, Yan SF, Schmidt AM. Receptor for advanced glycation endproducts (rage) and the complications of diabetes. Ageing Res Rev. 2002;1:1-15

30. Shinohara M, Thornalley PJ, Giardino I, Beisswenger P, Thorpe SR, Onorato J, et al. Overexpression of glyoxalase-i in bovine endothelial cells inhibits intracellular advanced glycation endproduct formation and prevents hyperglycemia-induced increases in macromolecular endocytosis. J Clin Invest. 1998;101:11421147

31. Monnier VM. Intervention against the maillard reaction in vivo. Arch Biochem Biophys. 2003;419:1-15

32. Nagai R, Murray, D.B., Metz, T.O., Baynes, J.W. Chelation: A fundamental mechanism of action of age inhibitors, age breakers, and other inhibitors of diabetes complications. Diabetes. 2012;61:549-559

33. Schalkwijk CG. Therapeutic interventions in the glyc(oxid)ation pathway. Immun Endoc \& Metab Agents in Med Chem. 2007;7:57-68

34. Sourris KC, Harcourt BE, Forbes JM. A new perspective on therapeutic inhibition of advanced glycation in diabetic microvascular complications: Common downstream endpoints achieved through disparate therapeutic approaches? Am J Nephrol. 2009;30:323-335

35. Peyroux J, Sternberg M. Advanced glycation endproducts (ages): Pharmacological inhibition in diabetes. Pathol Biol (Paris). 2006;54:405-419

36. Thornalley PJ. Use of aminoguanidine (pimagedine) to prevent the formation of advanced glycation endproducts. Arch Biochem Biophys. 2003;419:31-40

37. Brownlee $M$, Vlassara $H$, Kooney A, Ulrich $P$, Cerami A. Aminoguanidine prevents diabetes-induced arterial wall protein cross-linking. Science. 1986;232:1629-1632

38. Fu MX, Wells-Knecht KJ, Blackledge JA, Lyons TJ, Thorpe SR, Baynes JW. Glycation, glycoxidation, and crosslinking of collagen by glucose. Kinetics, mechanisms, and inhibition of late stages of the maillard reaction. Diabetes. 1994;43:676-683

39. Huijberts MS, Wolffenbuttel BH, Boudier HA, Crijns FR, Kruseman AC, Poitevin P, et al. Aminoguanidine treatment increases elasticity and decreases fluid filtration of large arteries from diabetic rats. $J$ Clin Invest. 1993;92:1407-1411

40. Birrell AM, Heffernan SJ, Kirwan P, McLennan S, Gillin AG, Yue DK. The effects of aminoguanidine on renal changes in a baboon model of type 1 diabetes. J Diabetes Complications. 2002;16:301-309

41. Edelstein D, Brownlee M. Aminoguanidine ameliorates albuminuria in diabetic hypertensive rats. Diabetologia. 1992;35:96-97

42. Yamauchi A, Takei I, Makita Z, Nakamoto S, Ohashi N, Kiguchi H, et al. Effects of aminoguanidine on serum advanced glycation endproducts, urinary albilmin excretion, mesangial expansion, and glomerular basement membrane thickening in otsuka long-evans tokushima fatty rats. Diabetes Res Clin Pract. 1997;34:127-133

43. Soulis-Liparota T, Cooper M, Papazoglou D, Clarke B, Jerums G. Retardation by aminoguanidine of development of albuminuria, mesangial expansion, and tissue fluorescence in streptozocin-induced diabetic rat. Diabetes. 1991;40:1328-1334

44. Hammes HP, Martin S, Federlin K, Geisen K, Brownlee M. Aminoguanidine treatment inhibits the development of experimental diabetic retinopathy. Proc Natl Acad Sci U S A. 1991;88:11555-11558

45. Kern TS, Engerman RL. Pharmacological inhibition of diabetic retinopathy: Aminoguanidine and aspirin. Diabetes. 2001;50:1636-1642

46. Miyauchi $\mathrm{Y}$, Shikama H, Takasu T, Okamiya H, Umeda M, Hirasaki E, et al. Slowing of peripheral motor nerve conduction was ameliorated by aminoguanidine in streptozocin-induced diabetic rats. Eur J Endocrinol. 1996;134:467-473 
47. Schmidt RE, Dorsey DA, Beaudet LN, Reiser KM, Williamson JR, Tilton RG. Effect of aminoguanidine on the frequency of neuroaxonal dystrophy in the superior mesenteric sympathetic autonomic ganglia of rats with streptozocin-induced diabetes. Diabetes. 1996;45:284-290

48. Asif M, Egan J, Vasan S, Jyothirmayi GN, Masurekar MR, Lopez S, et al. An advanced glycation endproduct cross-link breaker can reverse age-related increases in myocardial stiffness. Proc Natl Acad Sci U S A. 2000;97:2809-2813

49. Candido R, Forbes JM, Thomas MC, Thallas V, Dean RG, Burns WC, et al. A breaker of advanced glycation end products attenuates diabetes-induced myocardial structural changes. Circ Res. 2003;92:785-792

50. Vaitkevicius PV, Lane M, Spurgeon H, Ingram DK, Roth GS, Egan JJ, et al. A cross-link breaker has sustained effects on arterial and ventricular properties in older rhesus monkeys. Proc Natl Acad Sci U S A. 2001;98:1171-1175

51. Vasan S, Zhang X, Zhang X, Kapurniotu A, Bernhagen J, Teichberg S, et al. An agent cleaving glucose-derived protein crosslinks in vitro and in vivo. Nature. 1996;382:275-278

52. Wolffenbuttel BH, Boulanger CM, Crijns FR, Huijberts MS, Poitevin P, Swennen GN, et al. Breakers of advanced glycation end products restore large artery properties in experimental diabetes. Proc Natl Acad Sci U S A. 1998;95:4630-4634

53. Forbes JM, Yee LT, Thallas V, Lassila M, Candido R, Jandeleit-Dahm KA, et al. Advanced glycation end product interventions reduce diabetes-accelerated atherosclerosis. Diabetes. 2004;53:1813-1823

54. Forbes JM, Thallas V, Thomas MC, Founds HW, Burns WC, Jerums G, et al. The breakdown of preexisting advanced glycation end products is associated with reduced renal fibrosis in experimental diabetes. Faseb $\mathrm{J}$. 2003;17:1762-1764

55. Lassila M, Seah KK, Allen TJ, Thallas V, Thomas MC, Candido R, et al. Accelerated nephropathy in diabetic apolipoprotein e-knockout mouse: Role of advanced glycation end products. J Am Soc Nephrol. 2004; 15:2125-2138

56. Thallas-Bonke V, Lindschau C, Rizkalla B, Bach LA, Boner G, Meier M, et al. Attenuation of extracellular matrix accumulation in diabetic nephropathy by the advanced glycation end product cross-link breaker alt711 via a protein kinase c-alpha-dependent pathway. Diabetes. 2004;53:2921-2930

57. Booth AA, Khalifah RG, Hudson BG. Thiamine pyrophosphate and pyridoxamine inhibit the formation of antigenic advanced glycation end-products: Comparison with aminoguanidine. Biochem Biophys Res Commun. 1996;220:113-119

58. Booth AA, Khalifah RG, Todd P, Hudson BG. In vitro kinetic studies of formation of antigenic advanced glycation end products (ages). Novel inhibition of post-amadori glycation pathways. $J$ Biol Chem. 1997;272:5430-5437

59. Alkhalaf A, Klooster A, van Oeveren W, Achenbach U, Kleefstra N, Slingerland RJ, et al. A double-blind, randomized, placebo-controlled clinical trial on benfotiamine treatment in patients with diabetic nephropathy. Diabetes Care. 2010;33:1598-1601

60. Degenhardt TP, Alderson NL, Arrington DD, Beattie RJ, Basgen JM, Steffes MW, et al. Pyridoxamine inhibits early renal disease and dyslipidemia in the streptozotocin-diabetic rat. Kidney Int. 2002;61:939-950

61. Hammes HP, Du X, Edelstein D, Taguchi T, Matsumura T, Ju Q, et al. Benfotiamine blocks three major pathways of hyperglycemic damage and prevents experimental diabetic retinopathy. Nat Med. 2003;9:294299

62. Stitt A, Gardiner TA, Alderson NL, Canning P, Frizzell N, Duffy N, et al. The age inhibitor pyridoxamine inhibits development of retinopathy in experimental diabetes. Diabetes. 2002;51:2826-2832

63. Babaei-Jadidi R, Karachalias N, Ahmed N, Battah S, Thornalley PJ. Prevention of incipient diabetic nephropathy by high-dose thiamine and benfotiamine. Diabetes. 2003;52:2110-2120

64. Balakumar P, Chakkarwar VA, Singh M. Ameliorative effect of combination of benfotiamine and fenofibrate in diabetes-induced vascular endothelial dysfunction and nephropathy in the rat. Mol Cell Biochem. 2009;320:149-162

65. Karachalias N, Babaei-Jadidi R, Rabbani N, Thornalley PJ. Increased protein damage in renal glomeruli, retina, nerve, plasma and urine and its prevention by thiamine and benfotiamine therapy in a rat model of diabetes. Diabetologia. 2010;53:1506-1516

66. Bolton WK, Cattran DC, Williams ME, Adler SG, Appel GB, Cartwright K, et al. Randomized trial of an inhibitor of formation of advanced glycation end products in diabetic nephropathy. Am $J$ Nephrol. $2004 ; 24: 32-40$ 
67. Freedman BI, Wuerth JP, Cartwright K, Bain RP, Dippe S, Hershon K, et al. Design and baseline characteristics for the aminoguanidine clinical trial in overt type 2 diabetic nephropathy (action ii). Control Clin Trials. 1999;20:493-510

68. Hartog JW, Willemsen S, van Veldhuisen DJ, Posma JL, van Wijk LM, Hummel YM, et al. Effects of alagebrium, an advanced glycation endproduct breaker, on exercise tolerance and cardiac function in patients with chronic heart failure. Eur J Heart Fail. 2011;13:899-908

69. Kass DA, Shapiro EP, Kawaguchi M, Capriotti AR, Scuteri A, deGroof RC, et al. Improved arterial compliance by a novel advanced glycation end-product crosslink breaker. Circulation. 2001;104:1464-1470

70. Zieman SJ, Melenovsky V, Clattenburg L, Corretti MC, Capriotti A, Gerstenblith G, et al. Advanced glycation endproduct crosslink breaker (alagebrium) improves endothelial function in patients with isolated systolic hypertension. J Hypertens. 2007; 25:577-583

71. Little WC, Zile MR, Kitzman DW, Hundley WG, O'Brien TX, Degroof RC. The effect of alagebrium chloride (alt-711), a novel glucose cross-link breaker, in the treatment of elderly patients with diastolic heart failure. J Card Fail. 2005;11:191-195

72. Williams ME, Bolton WK, Khalifah RG, Degenhardt TP, Schotzinger RJ, McGill JB. Effects of pyridoxamine in combined phase 2 studies of patients with type 1 and type 2 diabetes and overt nephropathy. Am J Nephrol. 2007;27:605-614

73. Nascimento MM, Suliman ME, Murayama Y, Nihi M, Hayashi SY, Stenvinkel P, et al. Effect of high-dose thiamine and pyridoxine on advanced glycation end products and other oxidative stress markers in hemodialysis patients: A randomized placebo-controlled study. J Ren Nutr. 2006;16:119-124

74. Schupp N, Dette EM, Schmid U, Bahner U, Winkler M, Heidland A, et al. Benfotiamine reduces genomic damage in peripheral lymphocytes of hemodialysis patients. Naunyn Schmiedebergs Arch Pharmacol. 2008;378:283-291

75. Stirban A, Negrean M, Stratmann B, Gawlowski T, Horstmann T, Gotting C, et al. Benfotiamine prevents macro- and microvascular endothelial dysfunction and oxidative stress following a meal rich in advanced glycation end products in individuals with type 2 diabetes. Diabetes Care. 2006;29:2064-2071

76. Yao D, Taguchi T, Matsumura T, Pestell R, Edelstein D, Giardino I, et al. High glucose increases angiopoietin2 transcription in microvascular endothelial cells through methylglyoxal modification of msin3a. J Biol Chem. 2007;282:31038-31045

77. Du X, Edelstein D, Brownlee M. Oral benfotiamine plus alpha-lipoic acid normalises complication-causing pathways in type 1 diabetes. Diabetologia. 2008;51:1930-1932

78. Kawai T, Takei I, Tokui M, Funae O, Miyamoto K, Tabata M, et al. Effects of epalrestat, an aldose reductase inhibitor, on diabetic peripheral neuropathy in patients with type 2 diabetes, in relation to suppression of n(varepsilon)-carboxymethyl lysine. J Diabetes Complications. 2010;24:424-432

79. Nakamura N, Yamazaki K, Satoh A, Urakaze M, Kobayashi M, Yamabe H, et al. Effects of eparlestat on plasma levels of advanced glycation end products in patients with type 2 diabetes. In Vivo. 2003;17:177-180

80. Hamada Y, Nakamura J, Naruse K, Komori T, Kato K, Kasuya Y, et al. Epalrestat, an aldose reductase ihibitor, reduces the levels of nepsilon-(carboxymethyl)lysine protein adducts and their precursors in erythrocytes from diabetic patients. Diabetes Care. 2000;23:1539-1544

81. Hamada Y, Odagaki Y, Sakakibara F, Naruse K, Koh N, Hotta N. Effects of an aldose reductase inhibitor on erythrocyte fructose 3-phosphate and sorbitol 3-phosphate levels in diabetic patients. Life Sci. 1995;57:2329

82. Beisswenger PJ, Howell SK, Touchette AD, Lal S, Szwergold BS. Metformin reduces systemic methylglyoxal levels in type 2 diabetes. Diabetes. 1999;48:198-202

83. Rabbani N, Chittari MV, Bodmer CW, Zehnder D, Ceriello A, Thornalley PJ. Increased glycation and oxidative damage to apolipoprotein b100 of Idl cholesterol in patients with type 2 diabetes and effect of metformin. Diabetes. 2010;59:1038-1045

84. Faure P, Wiernsperger N, Polge C, Favier A, Halimi S. Impairment of the antioxidant properties of serum albumin in patients with diabetes: Protective effects of metformin. Clin Sci (Lond). 2008;114:251-256

85. Engelen L, Lund SS, Ferreira I, Tarnow L, Parving HH, Gram J, et al. Improved glycemic control induced by both metformin and repaglinide is associated with a reduction in blood levels of 3-deoxyglucosone in nonobese patients with type 2 diabetes. Eur J Endocrinol. 2011;164:371-379

86. Lund SS, Tarnow L, Stehouwer CD, Schalkwijk CG, Teerlink T, Gram J, et al. Impact of metformin versus repaglinide on non-glycaemic cardiovascular risk markers related to inflammation and endothelial dysfunction in non-obese patients with type 2 diabetes. Eur J Endocrinol. 2008;158:631-641 
87. Kanazawa I, Yamamoto M, Yamaguchi T, Sugimoto T. Effects of metformin and pioglitazone on serum pentosidine levels in type 2 diabetes mellitus. Exp Clin Endocrinol Diabetes. 2011;119:362-365

88. Yu Y, Thorpe SR, Jenkins AJ, Shaw JN, Sochaski MA, McGee D, et al. Advanced glycation end-products and methionine sulphoxide in skin collagen of patients with type 1 diabetes. Diabetologia. 2006;49:2488-2498

89. Monnier VM, Bautista O, Kenny D, Sell DR, Fogarty J, Dahms W, et al. Skin collagen glycation, glycoxidation, and crosslinking are lower in subjects with long-term intensive versus conventional therapy of type 1 diabetes: Relevance of glycated collagen products versus hba1c as markers of diabetic complications. Dcct skin collagen ancillary study group. Diabetes control and complications trial. Diabetes. 1999;48:870-880

90. Norlinah MI, Hamizah R, Md Isa SH, Wan Nazaimoon WM, Khalid BA. The effects of short-term, rapid glycemic control on the peroneal nerve function and serum vcam-1 and age in type 2 diabetic patients in malaysia. Indian J Med Sci. 2009;63:131-138

91. Jaleel A, Halvatsiotis P, Williamson B, Juhasz P, Martin S, Nair KS. Identification of amadori-modified plasma proteins in type 2 diabetes and the effect of short-term intensive insulin treatment. Diabetes Care. 2005;28:645-652

92. Salmela PI, Oikarinen Al, Ukkola O, Karjalainen A, Linnaluoto M, Puukka R, et al. Improved metabolic control in patients with non-insulin-dependent diabetes mellitus is associated with a slower accumulation of glycation products in collagen. Eur J Clin Invest. 1995;25:494-500

93. Schiel R, Franke S, Appel T, Voigt U, Ross IS, Kientsch-Engel R, et al. Improvement of the quality of diabetes control and decrease in the concentrations of age-products in patients with type 1 and insulin-treated type 2 diabetes mellitus: Results from a 10 year-prospective, population-based survey on the quality of diabetes care in germany (jevin). Eur J Med Res. 2004;9:391-399

94. Schiel R, Franke S, Appel T, Voigt U, Ross IS, Kientsch-Engel R, et al. Improvement in quality of diabetes control and concentrations of age-products in patients with type 1 and insulin-treated type 2 diabetes mellitus studied over a period of 10 years (jevin). J Diabetes Complications. 2003;17:90-97

95. Chiarelli F, de Martino M, Mezzetti A, Catino M, Morgese G, Cuccurullo F, et al. Advanced glycation end products in children and adolescents with diabetes: Relation to glycemic control and early microvascular complications. J Pediatr. 1999;134:486-491

96. Lyons TJ, Bailie KE, Dyer DG, Dunn JA, Baynes JW. Decrease in skin collagen glycation with improved glycemic control in patients with insulin-dependent diabetes mellitus. J Clin Invest. 1991;87:1910-1915

97. Mentink CJ, Kilhovd BK, Rondas-Colbers GJ, Torjesen PA, Wolffenbuttel BH. Time course of specific ages during optimised glycaemic control in type 2 diabetes. Neth J Med. 2006;64:10-16

98. Busch M, Franke S, Wolf G, Rohde RD, Stein G. Serum levels of the advanced glycation end products nepsilon-carboxymethyllysine and pentosidine are not influenced by treatment with the angiotensin receptor ii type 1 blocker irbesartan in patients with type 2 diabetic nephropathy and hypertension. Nephron Clin Pract. 2008;108:c291-297

99. Engelen L, Persson F, Ferreira I, Rossing P, Hovind P, Teerlink T, et al. Irbesartan treatment does not influence plasma levels of the advanced glycation end products $n$ (epsilon)(1-carboxymethyl)lysine and n(epsilon)(1-carboxyethyl)lysine in patients with type 2 diabetes and microalbuminuria. A randomized controlled trial. Nephrol Dial Transplant. 2011;26:3573-3577

100. Persson F, Rossing P, Hovind P, Stehouwer CD, Schalkwijk C, Tarnow L, et al. Irbesartan treatment reduces biomarkers of inflammatory activity in patients with type 2 diabetes and microalbuminuria: An irma 2 substudy. Diabetes. 2006;55:3550-3555

101. Hartog JW, van de Wal RM, Schalkwijk CG, Miyata T, Jaarsma W, Plokker HW, et al. Advanced glycation endproducts, anti-hypertensive treatment and diastolic function in patients with hypertension and diastolic dysfunction. Eur J Heart Fail. 2010

102. Mitsuhashi H, Tamura K, Yamauchi J, Ozawa M, Yanagi M, Dejima T, et al. Effect of losartan on ambulatory short-term blood pressure variability and cardiovascular remodeling in hypertensive patients on hemodialysis. Atherosclerosis. 2009;207:186-190

103. Saha SA, LaSalle BK, Clifton GD, Short RA, Tuttle KR. Modulation of advanced glycation end products by candesartan in patients with diabetic kidney disease--a dose-response relationship study. Am J Ther. 2010;17:553-558

104. Komiya N, Hirose H, Saisho Y, Saito I, Itoh H. Effects of 12-month valsartan therapy on glycation and oxidative stress markers in type 2 diabetic subjects with hypertension. Int Heart J. 2008;49:681-689

105. Monacelli F, Poggi A, Storace D, Durante A, Traverso N, Viviani GL, et al. Effects of valsartan therapy on protein glycoxidation. Metabolism. 2006;55:1619-1624 
106. Saisho Y, Komiya N, Hirose H. Effect of valsartan, an angiotensin ii receptor blocker, on markers of oxidation and glycation in japanese type 2 diabetic subjects: Blood pressure-independent effect of valsartan. Diabetes Res Clin Pract. 2006;74:201-203

107. Rabbani N, Adaikalakoteswari A, Rossing K, Rossing P, Tarnow L, Parving HH, et al. Effect of irbesartan treatment on plasma and urinary markers of protein damage in patients with type 2 diabetes and microalbuminuria. Amino Acids. 2011

108. Sebekova K, Gazdikova K, Syrova D, Blazicek P, Schinzel R, Heidland A, et al. Effects of ramipril in nondiabetic nephropathy: Improved parameters of oxidatives stress and potential modulation of advanced glycation end products. J Hum Hypertens. 2003;17:265-270

109. Nakamura T, Sato E, Fujiwara N, Kawagoe $Y$, Koide H, Ueda Y, et al. Calcium channel blocker inhibition of age and rage axis limits renal injury in nondiabetic patients with stage $\mathrm{i}$ or ii chronic kidney disease. Clin Cardiol. 2011;34:372-377

110. Scharnagl H, Stojakovic T, Winkler K, Rosinger S, Marz W, Boehm BO. The hmg-coa reductase inhibitor cerivastatin lowers advanced glycation end products in patients with type 2 diabetes. Exp Clin Endocrinol Diabetes. 2007;115:372-375

111. Tam HL, Shiu SW, Wong Y, Chow WS, Betteridge DJ, Tan KC. Effects of atorvastatin on serum soluble receptors for advanced glycation end-products in type 2 diabetes. Atherosclerosis. 2010;209:173-177

112. Cuccurullo C, lezzi A, Fazia ML, De Cesare D, Di Francesco A, Muraro R, et al. Suppression of rage as a basis of simvastatin-dependent plaque stabilization in type 2 diabetes. Arterioscler Thromb Vasc Biol. 2006;26:2716-2723

113. Jinnouchi $Y$, Yamagishi S, Takeuchi M, Ishida S, Jinnouchi $Y$, Jinnouchi J, et al. Atorvastatin decreases serum levels of advanced glycation end products (ages) in patients with type 2 diabetes. Clin Exp Med. 2006;6:191193

114. Kimura Y, Hyogo H, Yamagishi S, Takeuchi M, Ishitobi T, Nabeshima Y, et al. Atorvastatin decreases serum levels of advanced glycation endproducts (ages) in nonalcoholic steatohepatitis (nash) patients with dyslipidemia: Clinical usefulness of ages as a biomarker for the attenuation of nash. J Gastroenterol. 2010;45:750-757

115. Kageyama Y, Takahashi M, Ichikawa T, Torikai E, Nagano A. Reduction of oxidative stress marker levels by anti-tnf-alpha antibody, infliximab, in patients with rheumatoid arthritis. Clin Exp Rheumatol. 2008;26:73-80

116. Kageyama Y, Takahashi M, Nagafusa T, Torikai E, Nagano A. Etanercept reduces the oxidative stress marker levels in patients with rheumatoid arthritis. Rheumatol Int. 2008;28:245-251

117. Kageyama Y, Takahashi M, Nagafusa T, Torikai E, Nagano A. Methotrexate reduces the levels of pentosidine and 8-hydroxy-deoxy guanosine in patients with rheumatoid arthritis. Mod Rheumatol. 2007;17:398-402

118. Diamanti-Kandarakis E, Katsikis I, Piperi C, Alexandraki K, Panidis D. Effect of long-term orlistat treatment on serum levels of advanced glycation end-products in women with polycystic ovary syndrome. Clin Endocrinol (Oxf). 2007;66:103-109

119. Gugliucci A, Kotani K, Taing J, Matsuoka Y, Sano Y, Yoshimura M, et al. Short-term low calorie diet intervention reduces serum advanced glycation end products in healthy overweight or obese adults. Ann Nutr Metab. 2009;54:197-201

120. Iwashige K, Kouda K, Kouda M, Horiuchi K, Takahashi M, Nagano A, et al. Calorie restricted diet and urinary pentosidine in patients with rheumatoid arthritis. J Physiol Anthropol Appl Human Sci. 2004;23:19-24

121. Yoshikawa T, Miyazaki A, Fujimoto S. Decrease in serum levels of advanced glycation end-products by shortterm lifestyle modification in non-diabetic middle-aged females. Med Sci Monit. 2009;15:PH65-73

122. Diamanti-Kandarakis E, Piperi C, Alexandraki K, Katsilambros N, Kouroupi E, Papailiou J, et al. Short-term effect of orlistat on dietary glycotoxins in healthy women and women with polycystic ovary syndrome. Metabolism. 2006;55:494-500

123. Diamanti-Kandarakis E, Piperi C, Alexandraki KI, Papailiou J, Ekonomou F, Koulouri E, et al. The acute effect of orlistat on dietary glycotoxins in diabetics and healthy women. Minerva Endocrinol. 2009;34:97-104

124. Cai W, He JC, Zhu L, Peppa M, Lu C, Uribarri J, et al. High levels of dietary advanced glycation end products transform low-density lipoprotein into a potent redox-sensitive mitogen-activated protein kinase stimulant in diabetic patients. Circulation. 2004;110:285-291

125. Uribarri J, Cai W, Ramdas M, Goodman S, Pyzik R, Chen X, et al. Restriction of advanced glycation end products improves insulin resistance in human type 2 diabetes: Potential role of ager1 and sirt1. Diabetes Care. 2011;34:1610-1616

126. Uribarri J, Peppa M, Cai W, Goldberg T, Lu M, He C, et al. Restriction of dietary glycotoxins reduces excessive advanced glycation end products in renal failure patients. J Am Soc Nephrol. 2003;14:728-731 
127. Vlassara H, Cai W, Goodman S, Pyzik R, Yong A, Chen X, et al. Protection against loss of innate defenses in adulthood by low advanced glycation end products (age) intake: Role of the antiinflammatory age receptor1. J Clin Endocrinol Metab. 2009;94:4483-4491

128. Jackson $S$, James $M$, Abrams $P$. The effect of oestradiol on vaginal collagen metabolism in postmenopausal women with genuine stress incontinence. BJOG. 2002;109:339-344

129. Sundaram RC, Selvaraj N, Vijayan G, Bobby Z, Hamide A, Rattina Dasse N. Increased plasma malondialdehyde and fructosamine in iron deficiency anemia: Effect of treatment. Biomed Pharmacother. 2007;61:682-685

130. Vijayan G, Sundaram RC, Bobby Z, Hamide A, Selvaraj N, Dasse NR. Increased plasma malondialdehyde and fructosamine in anemic $h$ pylori infected patients: Effect of treatment. World J Gastroenterol. 2007;13:796800

131. Brignardello E, Runzo C, Aragno M, Catalano MG, Cassader M, Perin PC, et al. Dehydroepiandrosterone administration counteracts oxidative imbalance and advanced glycation end product formation in type 2 diabetic patients. Diabetes Care. 2007;30:2922-2927

132. Contreras I, Reiser KM, Martinez N, Giansante E, Lopez T, Suarez N, et al. Effects of aspirin or basic amino acids on collagen cross-links and complications in niddm. Diabetes Care. 1997;20:832-835

133. You WH, Wang P, Li MQ, Zhang Y, Peng YL, Zhang FL. Therapeutic effects of modified danggui sini decoction on plasma level of advanced glycation end products in patients with wagner grade 0 diabetic foot: $A$ randomized controlled trial. Zhong Xi Yi Jie He Xue Bao. 2009;7:622-628

134. Kakuta T, Tanaka R, Hyodo T, Suzuki H, Kanai G, Nagaoka M, et al. Effect of sevelamer and calcium-based phosphate binders on coronary artery calcification and accumulation of circulating advanced glycation end products in hemodialysis patients. Am J Kidney Dis. 2011;57:422-431

135. Gomez-Perez FJ, Valles-Sanchez VE, Lopez-Alvarenga JC, Choza-Romero R, Ibarra Pascuali JJ, Gonzalez Orellana R, et al. Vitamin e modifies neither fructosamine nor hba1c levels in poorly controlled diabetes. Rev Invest Clin. 1996;48:421-424

136. Lu L, Erhard P, Salomon RG, Weiss MF. Serum vitamin e and oxidative protein modification in hemodialysis: A randomized clinical trial. Am J Kidney Dis. 2007;50:305-313

137. Reaven PD, Herold DA, Barnett J, Edelman S. Effects of vitamin e on susceptibility of low-density lipoprotein and low-density lipoprotein subfractions to oxidation and on protein glycation in niddm. Diabetes Care. 1995;18:807-816

138. Morano S, Mandosi E, Fallarino M, Gatti A, Tiberti C, Sensi M, et al. Antioxidant treatment associated with sildenafil reduces monocyte activation and markers of endothelial damage in patients with diabetic erectile dysfunction: A double-blind, placebo-controlled study. Eur Urol. 2007;52:1768-1774

139. Suji G, Sivakami S. DNA damage by free radical production by aminoguanidine. Ann N Y Acad Sci. 2006;1067:191-199

140. Tilton RG, Chang K, Hasan KS, Smith SR, Petrash JM, Misko TP, et al. Prevention of diabetic vascular dysfunction by guanidines. Inhibition of nitric oxide synthase versus advanced glycation end-product formation. Diabetes. 1993;42:221-232

141. Turgut F, Bolton WK. Potential new therapeutic agents for diabetic kidney disease. Am J Kidney Dis. 2010;55:928-940

142. ClinicalTrials.gov. Registry and results database of federally and privately supported clinical trials conducted in the united states and around the world. 2012;Accessed February 2012

143. Voziyan PA, Khalifah RG, Thibaudeau C, Yildiz A, Jacob J, Serianni AS, et al. Modification of proteins in vitro by physiological levels of glucose: Pyridoxamine inhibits conversion of amadori intermediate to advanced glycation end-products through binding of redox metal ions. J Biol Chem. 2003;278:46616-46624

144. Lewis EJ, Greene T, Spitalewiz S, Blumenthal S, Berl T, Hunsicker LG, et al. Pyridorin in type 2 diabetic nephropathy. J Am Soc Nephrol. 2011

145. La Selva M, Beltramo E, Pagnozzi F, Bena E, Molinatti PA, Molinatti GM, et al. Thiamine corrects delayed replication and decreases production of lactate and advanced glycation end-products in bovine retinal and human umbilical vein endothelial cells cultured under high glucose conditions. Diabetologia. 1996;39:12631268

146. Brouwers O, Niessen PMG, Miyata T, Teerlink T, Janssen BJ, De Mey JGR, et al. Overexpression of glyoxalase-i improves vascular function in a rat model of diabetes. Diabetologia. 2010;53:S520

147. Sihag B, Meena B, Agrawal R, Meel J, Sirohi P, Bishnoi P. High dose thiamine therapy for patients with type 2 diabetes and microalbuminuria: A randomised, double-blind placebo-controlled study. Diabetes Obes Metab. 2010;12:87-88 
148. Ruggiero-Lopez D, Lecomte M, Moinet G, Patereau G, Lagarde M, Wiernsperger N. Reaction of metformin with dicarbonyl compounds. Possible implication in the inhibition of advanced glycation end product formation. Biochem Pharmacol. 1999;58:1765-1773

149. UK Prospective Diabetes Study (UKPDS) Group. Effect of intensive blood-glucose control with metformin on complications in overweight patients with type 2 diabetes (ukpds 34). Uk prospective diabetes study (ukpds) group. Lancet. 1998;352:854-865

150. Diamanti-Kandarakis E, Alexandraki K, Piperi C, Aessopos A, Paterakis T, Katsikis I, et al. Effect of metformin administration on plasma advanced glycation end product levels in women with polycystic ovary syndrome. Metabolism. 2007;56:129-134

151. Brenner BM, Cooper ME, de Zeeuw D, Keane WF, Mitch WE, Parving HH, et al. Effects of losartan on renal and cardiovascular outcomes in patients with type 2 diabetes and nephropathy. $N$ Engl J Med. 2001;345:861-869

152. Parving HH, Lehnert H, Brochner-Mortensen J, Gomis R, Andersen S, Arner P. The effect of irbesartan on the development of diabetic nephropathy in patients with type 2 diabetes. N Engl J Med. 2001;345:870-878

153. Miyata T, van Ypersele de Strihou C, Ueda Y, Ichimori K, Inagi R, Onogi $H$, et al. Angiotensin ii receptor antagonists and angiotensin-converting enzyme inhibitors lower in vitro the formation of advanced glycation end products: Biochemical mechanisms. J Am Soc Nephrol. 2002;13:2478-2487

154. Forbes JM, Thomas MC, Thorpe SR, Alderson NL, Cooper ME. The effects of valsartan on the accumulation of circulating and renal advanced glycation end products in experimental diabetes. Kidney Int Suppl. 2004:S105-107

155. Nangaku M, Miyata T, Sada T, Mizuno M, Inagi R, Ueda $Y$, et al. Anti-hypertensive agents inhibit in vivo the formation of advanced glycation end products and improve renal damage in a type 2 diabetic nephropathy rat model. J Am Soc Nephrol. 2003;14:1212-1222

156. Fan Q, Liao J, Kobayashi M, Yamashita M, Gu L, Gohda T, et al. Candesartan reduced advanced glycation end-products accumulation and diminished nitro-oxidative stress in type 2 diabetic kk/ta mice. Nephrol Dial Transplant. 2004;19:3012-3020

157. Izuhara $Y$, Sada T, Yanagisawa $H$, Koike $H$, Ohtomo S, Dan $T$, et al. A novel sartan derivative with very low angiotensin ii type 1 receptor affinity protects the kidney in type 2 diabetic rats. Arterioscler Thromb Vasc Biol. 2008;28:1767-1773

158. Sugiyama T, Okuno T, Fukuhara M, Oku H, Ikeda T, Obayashi H, et al. Angiotensin ii receptor blocker inhibits abnormal accumulation of advanced glycation end products and retinal damage in a rat model of type 2 diabetes. Exp Eye Res. 2007;85:406-412

159. Thomas MC, Tikellis C, Burns WM, Bialkowski K, Cao Z, Coughlan MT, et al. Interactions between renin angiotensin system and advanced glycation in the kidney. J Am Soc Nephrol. 2005;16:2976-2984

160. Forbes JM, Cooper ME, Thallas V, Burns WC, Thomas MC, Brammar GC, et al. Reduction of the accumulation of advanced glycation end products by ace inhibition in experimental diabetic nephropathy. Diabetes. 2002;51:3274-3282

161. Liu XP, Pang YJ, Zhu WW, Zhao TT, Zheng M, Wang YB, et al. Benazepril, an angiotensin-converting enzyme inhibitor, alleviates renal injury in spontaneously hypertensive rats by inhibiting advanced glycation endproduct-mediated pathways. Clin Exp Pharmacol Physiol. 2009;36:287-296

162. Miyata T, van Ypersele de Strihou C. Angiotensin ii receptor blockers and angiotensin converting enzyme inhibitors: Implication of radical scavenging and transition metal chelation in inhibition of advanced glycation end product formation. Arch Biochem Biophys. 2003;419:50-54

163. Aviram M, Rosenblat M, Bisgaier CL, Newton RS. Atorvastatin and gemfibrozil metabolites, but not the parent drugs, are potent antioxidants against lipoprotein oxidation. Atherosclerosis. 1998;138:271-280

164. Akira K, Amano M, Okajima F, Hashimoto T, Oikawa S. Inhibitory effects of amlodipine and fluvastatin on the deposition of advanced glycation end products in aortic wall of cholesterol and fructose-fed rabbits. Biol Pharm Bull. 2006;29:75-81

165. Sezer ED, Sozmen EY, Nart D, Onat T. Effect of atorvastatin therapy on oxidant-antioxidant status and atherosclerotic plaque formation. Vasc Health Risk Manag. 2011;7:333-343

166. Scheijen JL, van de Waarenburg MP, Stehouwer CD, Schalkwijk CG. Measurement of pentosidine in human plasma protein by a single-column high-performance liquid chromatography method with fluorescence detection. J Chromatogr B Analyt Technol Biomed Life Sci. 2009;877:610-614

167. Teerlink T, Barto R, Ten Brink HJ, Schalkwijk CG. Measurement of nepsilon-(carboxymethyl)lysine and nepsilon-(carboxyethyl)lysine in human plasma protein by stable-isotope-dilution tandem mass spectrometry. Clin Chem. 2004;50:1222-1228 
168. Thornalley PJ, Battah S, Ahmed N, Karachalias N, Agalou S, Babaei-Jadidi R, et al. Quantitative screening of advanced glycation endproducts in cellular and extracellular proteins by tandem mass spectrometry. Biochem J. 2003;375:581-592

169. Tan KC, Chow WS, Tso AW, Xu A, Tse HF, Hoo RL, et al. Thiazolidinedione increases serum soluble receptor for advanced glycation end-products in type 2 diabetes. Diabetologia. 2007;50:1819-1825

170. Tsunosue M, Mashiko N, Ohta Y, Matsuo Y, Ueda K, Ninomiya M, et al. An alpha-glucosidase inhibitor, acarbose treatment decreases serum levels of glyceraldehyde-derived advanced glycation end products (ages) in patients with type 2 diabetes. Clin Exp Med. 2010;10:139-141

171. Beisswenger PJ, Howell SK, O'Dell RM, Wood ME, Touchette AD, Szwergold BS. Alpha-dicarbonyls increase in the postprandial period and reflect the degree of hyperglycemia. Diabetes Care. 2001;24:726-732

172. Ahmed N, Babaei-Jadidi R, Howell SK, Thornalley PJ, Beisswenger PJ. Glycated and oxidized protein degradation products are indicators of fasting and postprandial hyperglycemia in diabetes. Diabetes Care. 2005;28:2465-2471

173. Guevara-Aguirre J, Guevara-Aguirre M, Saavedra J, Bernstein G, Rosenbloom AL. Comparison of oral insulin spray and subcutaneous regular insulin at mealtime in type 1 diabetes. Diabetes Technol Ther. 2007;9:372376 


\section{APPENDIX}

Table A2.1 Potential AGE-inhibiting effect of aldose reductase inhibitors

\begin{tabular}{|c|c|c|c|c|c|c|c|}
\hline Ref. & Therapy & $\begin{array}{l}\text { Patient } \\
\text { characteristics }\end{array}$ & Design & Treatment arms $(n)$ & $\begin{array}{l}\text { Treatment } \\
\text { duration }\end{array}$ & $\begin{array}{l}\text { Glycation-related } \\
\text { outcome }\end{array}$ & Results \\
\hline 78 & Epalrestat & $\begin{array}{l}\text { T2DM, } \\
\text { neuropathy }\end{array}$ & $\begin{array}{l}\text { Open-label } \\
\text { nonrandomized } \\
\text { controlled trial }\end{array}$ & $\begin{array}{l}150 \mathrm{mg} \text { epalrestat } \\
(n=38), \text { control group } \\
(n=36)\end{array}$ & 1 year & Serum-CML (ELISA) & $\begin{array}{l}\text { Serum-CML } \downarrow \text { in epalrestat } \\
\text { vs. control group }\end{array}$ \\
\hline 79 & Epalrestat & $\begin{array}{l}\text { T2DM, } \\
\text { neuropathy }\end{array}$ & $\begin{array}{l}\text { Uncontrolled (single- } \\
\text { arm) study }\end{array}$ & $\begin{array}{l}150 \mathrm{mg} \text { epalrestat } \\
(n=9), \text { no control group }\end{array}$ & 6 months & Plasma-CML (ELISA) & No treatment effects* \\
\hline 80 & Epalrestat & T2DM & $\begin{array}{l}\text { 1: Observational study; } \\
\text { 2: uncontrolled (single- } \\
\text { arm) study }\end{array}$ & $\begin{array}{l}\text { 1: } 150 \text { mg epalrestat } \\
\text { ( } n=16), \text { control group } \\
\text { ( } n=38) ; \\
2: 150 \text { mg epalrestat } \\
\text { ( } n=14), \text { no control } \\
\text { group }\end{array}$ & $\begin{array}{l}1: \geq 2 \\
\text { months; } \\
2: 2 \text { months }\end{array}$ & $\begin{array}{l}1 \text { and 2: erythrocyte-CML } \\
\text { (ELISA) and 3DG (HPLC) }\end{array}$ & $\begin{array}{l}\text { 1: erythrocyte-CML and 3DG } \\
\downarrow \text { in epalrestat than in } \\
\text { control group; } \\
\text { 2: erythrocyte-CML and 3DG } \\
\downarrow \text { after epalrestat treatment }\end{array}$ \\
\hline 81 & Epalrestat & T2DM & $\begin{array}{l}\text { 1: Observational study; } \\
\text { 2: uncontrolled (single- } \\
\text { arm) study }\end{array}$ & $\begin{array}{l}\text { 1: } 150 \text { mg epalrestat } \\
(n=7), \text { control group } \\
(n=7) ; \\
\text { 2: } 150 \text { mg epalrestat } \\
(n=3), \text { no control group }\end{array}$ & $\begin{array}{l}\text { 1: } \geq 1 \text { month; } \\
2: 1 \text { month }\end{array}$ & $\begin{array}{l}1 \text { and 2: erythrocyte-S3P } \\
\text { and F3P (spectroscopy) }\end{array}$ & $\begin{array}{l}\text { 1: erythrocyte-S3P and F3P } \downarrow \\
\text { in epalrestat than in control } \\
\text { group; } \\
\text { 2: erythrocyte-S3P and F3P } \downarrow \\
\text { after epalrestat treatment }\end{array}$ \\
\hline
\end{tabular}

S3P, sorbitol 3-phosphate; F3P, fructose 3-phosphate; HPLC, high performance liquid chromatography; T2DM, type 2 diabetes.

*When a sub-group $(n=4)$ with baseline Plasma-CML $>3 \mathrm{mU} / \mathrm{mL}$ was analyzed separately, Plasma-CML $\downarrow$ after 3-month epalrestat treatment. 
Table A2.2 Potential AGE-inhibiting effects of glucose-lowering medication

\begin{tabular}{|c|c|c|c|c|c|c|c|}
\hline Ref. & Therapy & $\begin{array}{l}\text { Patient } \\
\text { characteristics }\end{array}$ & Design & Treatment arms $(n)$ & $\begin{array}{l}\text { Treatment } \\
\text { duration }\end{array}$ & Glycation-related outcome & Results \\
\hline 150 & Metformin & ND, PCOS & $\begin{array}{l}\text { Uncontrolled } \\
\text { (single-arm) } \\
\text { study }\end{array}$ & $\begin{array}{l}1700 \mathrm{mg} \text { metformin }(n=22) \text {, } \\
\text { no control group }\end{array}$ & 6 months & Serum-AGEs (ELISA) & $\begin{array}{l}\text { Serum-AGEs } \downarrow \text { after } \\
\text { metformin treatment }\end{array}$ \\
\hline 83 & Metformin & T2DM & $\begin{array}{l}\text { Observational } \\
\text { study }\end{array}$ & $\begin{array}{l}\text { Metformin }(n=13) \text {, control } \\
\text { group (on other } \\
\text { antihyperglycemic therapy; } \\
n=19 \text { ) }\end{array}$ & $1-20$ years & $\begin{array}{l}\text { LDL apoB100-FL, CML, CEL, } \\
\text { MG-H1, G-H1, 3DG-H, CMA, } \\
\text { MOLD and pentosidine (LC- } \\
\text { tandem MS) }\end{array}$ & $\begin{array}{l}\text { LDL apoB100-G-H1, MG-H1, } \\
\text { 3DG-H and CMA } \downarrow \text { in } \\
\text { metformin vs. control } \\
\text { group; no differences } \\
\text { between treatment groups } \\
\text { in LDL apoB100-CML, CEL, } \\
\text { MOLD and pentosidine }\end{array}$ \\
\hline 84 & Metformin & T2DM & $\begin{array}{l}\text { Observational } \\
\text { study }\end{array}$ & $\begin{array}{l}\text { Metformin }(n=23) \\
\text { sulfonylureas }(n=21)\end{array}$ & $\geq 2$ months & Serum-fructosamine & $\begin{array}{l}\text { Serum-fructosamine } \downarrow \text { in } \\
\text { metformin vs. sulfonylureas } \\
\text { group }\end{array}$ \\
\hline 82 & Metformin & T2DM & $\begin{array}{l}\text { Observational } \\
\text { study }\end{array}$ & $\begin{array}{l}1,000-2,500 \mathrm{mg} \text { metformin } \\
(n=13) ; 500-1,000 \mathrm{mg} \\
\text { metformin }(n=17)) \text {; control } \\
\text { group (on diet, } \\
\text { sulfonylureas or insulin; } \\
n=27)\end{array}$ & $\geq 3$ months & $\begin{array}{l}\text { Plasma-MG (HPLC) and 3DG } \\
\text { (GC-MS) }\end{array}$ & $\begin{array}{l}\text { Plasma-MG } \downarrow \text { in } 1,000 \text { - } \\
2,500 \text { mg metformin group } \\
\text { vs. } 500-1,000 \text { mg } \\
\text { metformin and T2DM } \\
\text { control group; no } \\
\text { differences between groups } \\
\text { on Plasma-3DG }\end{array}$ \\
\hline 85,86 & $\begin{array}{l}\text { Metformin } \\
\text { Repaglinide }\end{array}$ & $\begin{array}{l}\text { Non-obese } \\
\text { T2DM }\end{array}$ & $\begin{array}{l}\text { Double-blind } \\
\text { randomised } \\
\text { crossover study }\end{array}$ & $\begin{array}{l}2 \text { g metformin }(n=96), 6 \mathrm{mg} \\
\text { repaglinide }(n=96), \\
\text { crossover design }\end{array}$ & 4 months & $\begin{array}{l}\text { Blood-3DG (LC-MS/MS), } \\
\text { Plasma-CML, CEL (tandem- } \\
\text { MS), pyrraline (UPLC-tandem } \\
\text { MS) and AGE peptides } \\
\text { (spectrofluorometry) }\end{array}$ & $\begin{array}{l}\text { Blood-3DG } \downarrow \text { vs. baseline, } \\
\text { no effects on Plasma-CML, } \\
\text { CEL, pyrraline and AGE } \\
\text { peptides in both groups; no } \\
\text { between treatment effects }\end{array}$ \\
\hline 87 & $\begin{array}{l}\text { Metformin } \\
\text { Pioglitazone }\end{array}$ & T2DM & Open-label RCT & $\begin{array}{l}500-750 \mathrm{mg} \text { metformin } \\
(n=22), 15-30 \mathrm{mg} \\
\text { pioglitazone }(n=22), \text { control } \\
\text { group }(n=22)\end{array}$ & 6 months & Serum-pentosidine (ELISA) & $\begin{array}{l}\text { Serum-pentosidine } \downarrow \text { after } \\
\text { metformin and pioglitazone } \\
\text { vs. control group }\end{array}$ \\
\hline 169 & $\begin{array}{l}\text { Rosiglitazone } \\
\text { Sulfonylureas }\end{array}$ & T2DM & Open-label RCT & $\begin{array}{l}\text { 4-8 mg rosiglitazone }(n=32) \text {, } \\
5-10 \mathrm{mg} \text { glibenclamide or } \\
80-160 \mathrm{mg} \text { gliclazide }(n=32)\end{array}$ & 24 weeks & Serum-AGEs (ELISA) & $\begin{array}{l}\text { Serum-AGEs } \downarrow \text { vs. baseline } \\
\text { in both groups }\end{array}$ \\
\hline
\end{tabular}


Table A2.2 (continued)

\begin{tabular}{|c|c|c|c|c|c|c|c|}
\hline Ref. & Therapy & $\begin{array}{l}\text { Patient } \\
\text { characteristics }\end{array}$ & Design & Treatment arms ( $n)$ & $\begin{array}{l}\text { Treatment } \\
\text { duration }\end{array}$ & $\begin{array}{l}\text { Glycation-related } \\
\text { outcome }\end{array}$ & Results \\
\hline 170 & Acarbose & T2DM & $\begin{array}{l}\text { Uncontrolled } \\
\text { (single-arm) } \\
\text { study }\end{array}$ & $\begin{array}{l}150 \mathrm{mg} \text { acarbose } \\
(n=13), \text { no control } \\
\text { group }\end{array}$ & 12 weeks & Serum-AGEs (ELISA) & $\begin{array}{l}\text { Serum-AGEs } \downarrow \text { after acarbose } \\
\text { treatment }\end{array}$ \\
\hline $\begin{array}{l}171, \\
172\end{array}$ & Insulin lispro & T1DM & $\begin{array}{l}\text { Double-blind } \\
\text { crossover } \\
\text { study }\end{array}$ & $\begin{array}{l}\text { Insulin lispro }(n=21) \text {, } \\
\text { regular insulin } \\
(n=21), \text { crossover } \\
\text { design }\end{array}$ & 2 months & $\begin{array}{l}\text { Postprandial Plasma-MG } \\
\text { (HPLC), 3DG (GC-MS), } \\
\text { Urinary-fructosyl-lysine, } \\
\text { 3DG-H, MG-H1, G-H1, } \\
\text { CML, CEL, argpyrimidine, } \\
\text { MOLD and pentosidine } \\
\text { (LC-MS/MS) }\end{array}$ & $\begin{array}{l}\text { Postprandial Plasma-MG, 3DG, } \\
\text { CML, 3DG-H1 and Urinary-CML, } \\
\text { MG-H1, 3DG-H1 } \downarrow \text { in insulin lispro } \\
\text { vs. regular insulin group; no } \\
\text { treatment effects on Plasma- and } \\
\text { Urinary-fructosyl-lysine, CEL, G- } \\
\text { H1, MG-H1 and Urinary-arg- } \\
\text { pyrimidine, MOLD, pentosidine }\end{array}$ \\
\hline 173 & Oral insulin spray & T1DM & $\begin{array}{l}\text { Uncontrolled } \\
\text { (single-arm) } \\
\text { study }\end{array}$ & $\begin{array}{l}\text { Oral insulin spray } \\
(n=10) \text {, no control } \\
\text { group }\end{array}$ & 9 days & Serum-Fructosamine & No treatment effects \\
\hline 90 & $\begin{array}{l}\text { Intensified } \\
\text { antihyperglycaemic } \\
\text { treatment (insulin) }\end{array}$ & T2DM & $\begin{array}{l}\text { Single-blind } \\
\text { RCT }\end{array}$ & $\begin{array}{l}\text { Insulin ( } n=15 \text { ), } \\
\text { control group } \\
\text { (sulphonylureas, } \\
\text { metformin and/or } \\
\text { acarbose; } n=14 \text { ) }\end{array}$ & 8 weeks & $\begin{array}{l}\text { Serum-AGEs } \\
\text { (immunoassay), Serum- } \\
\text { fructosamine }\end{array}$ & $\begin{array}{l}\text { Serum-fructosamine } \downarrow \text { vs. } \\
\text { baseline, no effects on Serum- } \\
\text { AGEs; no between treatment } \\
\text { effects }\end{array}$ \\
\hline 88 & $\begin{array}{l}\text { Intensive antidiabetic } \\
\text { therapy (DCCT) }\end{array}$ & T1DM & $\begin{array}{l}\text { Open-label } \\
\text { RCT }\end{array}$ & $\begin{array}{l}\text { Intensive treatment } \\
(n=26), \text { conventional } \\
\text { treatment }(n=28)\end{array}$ & 6.5 years & $\begin{array}{l}\text { Skin-FL, CML (GC-MS) } \\
\text { and pentosidine (HPLC) }\end{array}$ & No treatment effects \\
\hline 89 & $\begin{array}{l}\text { Intensive antidiabetic } \\
\text { therapy (DCCT) }\end{array}$ & T1DM & $\begin{array}{l}\text { Open-label } \\
\text { RCT }\end{array}$ & $\begin{array}{l}\text { Intensive treatment } \\
(n=122) \text {, } \\
\text { conventional } \\
\text { treatment }(n=94)\end{array}$ & 5 years & $\begin{array}{l}\text { Skin-collagen glycation, } \\
\text { pentosidine, CML (HPLC) } \\
\text { and fluorescence }\end{array}$ & $\begin{array}{l}\text { Skin-collagen glycation, } \\
\text { pentosidine, CML and } \\
\text { fluorescence } \downarrow \text { in intensive vs. } \\
\text { conventional treatment group }\end{array}$ \\
\hline 97 & $\begin{array}{l}\text { Intensified } \\
\text { antihyperglycaemic } \\
\text { treatment (insulin) }\end{array}$ & T2DM & $\begin{array}{l}\text { Uncontrolled } \\
\text { (single-arm) } \\
\text { study }\end{array}$ & $\begin{array}{l}39 \pm 5 \cup \text { insulin } \\
(n=28), \text { no control } \\
\text { group }\end{array}$ & 6 months & $\begin{array}{l}\text { Serum-AGEs, MG-H1 } \\
\text { mmunoassay) and CML } \\
\text { (HPLC) }\end{array}$ & $\begin{array}{l}\text { Serum-CML (n.s.) } \downarrow \text { and Serum- } \\
\text { MG-H1 } \uparrow \text { after insulin treatment, } \\
\text { no effects on Serum-AGEs }\end{array}$ \\
\hline
\end{tabular}


Table A2.2 (continued)

\begin{tabular}{|c|c|c|c|c|c|c|c|}
\hline Ref. & Therapy & $\begin{array}{l}\text { Patient } \\
\text { characteristics }\end{array}$ & Design & Treatment arms $(n)$ & $\begin{array}{l}\text { Treatment } \\
\text { duration }\end{array}$ & $\begin{array}{l}\text { Glycation-related } \\
\text { outcome }\end{array}$ & Results \\
\hline 91 & $\begin{array}{l}\text { Intensified } \\
\text { antihyperglycaemic } \\
\text { treatment (insulin) }\end{array}$ & T2DM & $\begin{array}{l}\text { Uncontrolled } \\
\text { (single-arm) } \\
\text { study }\end{array}$ & $\begin{array}{l}\text { Insulin }(n=8) \text {, no control } \\
\text { group }\end{array}$ & 10 days & $\begin{array}{l}\text { Amadori-modified plasma } \\
\text { proteins (WB, MS), } \\
\text { Serum-fructosamine }\end{array}$ & $\begin{array}{l}\text { Serum-fructosamine and } \\
\text { Amadori-modified proteins } \downarrow \\
\text { after insulin treatment }\end{array}$ \\
\hline 93,94 & $\begin{array}{l}\text { Intensified } \\
\text { antihyperglycaemic } \\
\text { treatment (insulin) }\end{array}$ & T1DM or T2DM & $\begin{array}{l}\text { Uncontrolled } \\
\text { (single-arm) } \\
\text { study }\end{array}$ & $\begin{array}{l}\text { Intensified } \\
\text { antihyperglycaemic } \\
\text { treatment ( } n=120) \text {, no } \\
\text { control group }\end{array}$ & 5 years & $\begin{array}{l}\text { Serum-CML (ELISA) and } \\
\text { pentosidine (HPLC) }\end{array}$ & $\begin{array}{l}\text { Serum-CML and pentosidine } \\
\downarrow \text { after intensified } \\
\text { antihyperglycaemic } \\
\text { treatment }\end{array}$ \\
\hline 95 & $\begin{array}{l}\text { Intensified } \\
\text { antihyperglycaemic } \\
\text { treatment (insulin) }\end{array}$ & $\begin{array}{l}\text { T1DM (children } \\
\text { and } \\
\text { adolescents) }\end{array}$ & $\begin{array}{l}\text { Uncontrolled } \\
\text { (single-arm) } \\
\text { study }\end{array}$ & $\begin{array}{l}\text { Intensified insulin } \\
\text { therapy (to decrease } \\
\text { HbA1c form }>10 \% \text { to } \\
<7 \% \text {; } n=15 \text { ), no control } \\
\text { group }\end{array}$ & 2 years & $\begin{array}{l}\text { Serum-AGEs (DELFIA } \\
\text { fluorometer) }\end{array}$ & $\begin{array}{l}\text { Serum-AGEs } \downarrow \text { after } \\
\text { intensified insulin therapy }\end{array}$ \\
\hline 92 & $\begin{array}{l}\text { Intensified } \\
\text { antihyperglycaemic } \\
\text { treatment (mostly } \\
\text { insulin) }\end{array}$ & T2DM & $\begin{array}{l}\text { Uncontrolled } \\
\text { (single-arm) } \\
\text { study }\end{array}$ & $\begin{array}{l}\text { Intensified } \\
\text { antihyperglycaemic } \\
\text { treatment }(n=21) \text {, no } \\
\text { control group }\end{array}$ & 1.5 years & $\begin{array}{l}\text { Skin collagen glycation, } \\
\text { Serum-fructosamine }\end{array}$ & $\begin{array}{l}\text { Skin collagen glycation and } \\
\text { Serum-fructosamine } \downarrow \text { after } \\
\text { intensified } \\
\text { antihyperglycaemic } \\
\text { treatment }\end{array}$ \\
\hline 96 & $\begin{array}{l}\text { Intensified } \\
\text { antihyperglycaemic } \\
\text { treatment (insulin) }\end{array}$ & T1DM & $\begin{array}{l}\text { Uncontrolled } \\
\text { (single-arm) } \\
\text { study }\end{array}$ & $\begin{array}{l}52-88 \cup \text { insulin }(n=14) \text {, } \\
\text { no control group }\end{array}$ & 4 months & $\begin{array}{l}\text { Skin-FL, CML, CMhL (GC- } \\
\text { MS), pentosidine (HPLC), } \\
\text { skin fluorescence }\end{array}$ & $\begin{array}{l}\text { Skin-FL } \downarrow \text { after insulin } \\
\text { treatment; no effects on skin- } \\
\text { CML, CMhL, pentosidine and } \\
\text { fluorescence }\end{array}$ \\
\hline
\end{tabular}

$C M A, N^{\omega}$ (carboxymethyl)arginine; $C M h L, \mathrm{~N}^{\varepsilon}$ (carboxymethyl)hydroxylysine; $D C C T$, Diabetes control and complications trial; 3DG-H, 3-deoxyglucosone-derived hydroimidazolone; $F L$, fructoselysine; GC-MS, gas chromatography-mass spectrometry; $G-H 1$, glyoxal-derived hydroimidazolones; IgM, immunoglobulin M; MOLD, methylglyoxal lysine dimer; $M S$, mass spectrometry; ND, non-diabetic; PCOS, polycystic ovary syndrome; $R C T$, randomised controlled trial; $W B$, western blot. 
Table A2.3 Potential AGE-inhibiting effects of blood pressure-lowering medication

\begin{tabular}{|c|c|c|c|c|c|c|c|}
\hline Ref. & Therapy & $\begin{array}{l}\text { Patient } \\
\text { characteristics }\end{array}$ & Design & $\begin{array}{l}\text { Treatment arms } \\
\text { (n) }\end{array}$ & $\begin{array}{l}\text { Treatment } \\
\text { duration }\end{array}$ & Glycation-related outcome & Results \\
\hline 98 & Irbesartan & $\begin{array}{l}\text { T2DM, } \\
\text { nephropathy }\end{array}$ & $\begin{array}{l}\text { Double-blind } \\
\text { RCT }\end{array}$ & $\begin{array}{l}300 \text { mg irbesartan } \\
(n=65), \text { placebo } \\
(n=70)\end{array}$ & 2 years & $\begin{array}{l}\text { Serum-CML (ELISA) and } \\
\text { pentosidine (HPLC) }\end{array}$ & No treatment effects \\
\hline $\begin{array}{l}99, \\
100\end{array}$ & Irbesartan & T2DM, MA & $\begin{array}{l}\text { Double-blind } \\
\text { RCT }\end{array}$ & $\begin{array}{l}300 \text { mg irbesartan } \\
(n=139), \text { placebo } \\
(n=125)\end{array}$ & 2 years & $\begin{array}{l}\text { Plasma-CML, CEL (HPLC- } \\
\text { MS/MS) and AGES } \\
\text { (spectrofluorometry) }\end{array}$ & No treatment effects \\
\hline 101 & Eprosartan & $\begin{array}{l}\text { ND or DM, } \\
\text { diastolic } \\
\text { dysfunction }\end{array}$ & Open-label RCT & $\begin{array}{l}600 \text { mg eprosartan } \\
\text { on top of ( } n=47) \text { or } \\
\text { only ( } n=50) \text { other } \\
\text { BP-lowering drugs }\end{array}$ & 6 months & $\begin{array}{l}\text { Skin AF (in } n=26 \text { ), Plasma- } \\
\text { CML, CEL (LC-MS/MS) and } \\
\text { pentosidine (HPLC) }\end{array}$ & No treatment effects \\
\hline 102 & Losartan & $\begin{array}{l}\text { ND or DM, on } \\
\text { HD }\end{array}$ & Open-label RCT & $\begin{array}{l}25-100 \mathrm{mg} \\
\text { losartan }(n=20) \text {, } \\
\text { other BP-lowering } \\
\text { drugs }(n=20)\end{array}$ & $\begin{array}{l}12 \\
\text { months }\end{array}$ & Plasma-AGEs & $\begin{array}{l}\text { Plasma-AGEs } \downarrow \text { in losartan vs. control } \\
\text { group }\end{array}$ \\
\hline 107 & Irbesartan & T2DM & $\begin{array}{l}\text { Uncontrolled } \\
\text { study }\end{array}$ & $\begin{array}{l}300-900 \mathrm{mg} \\
\text { irbesartan ( } n=52) \text {, } \\
\text { no control group }\end{array}$ & 2 months & $\begin{array}{l}\text { Plasma- and Urinary- } \\
\text { pentosidine (HPLC), FL, CML, } \\
\text { CEL, G-H1, MG-H1, 3DG-H, } \\
\text { GOLD and MOLD (LC- } \\
\text { MS/MS) }\end{array}$ & $\begin{array}{l}\text { Plasma-3DG-H, Urinary-MG-H1 and G- } \\
\mathrm{H} 1 \downarrow \text { and Plasma-FL, CML, CEL and } \\
\text { pentosidine } \uparrow \text { after irbesartan } \\
\text { treatment; no treatment effects on all } \\
\text { other Plasma- and Urinary-AGEs }\end{array}$ \\
\hline 103 & Candesartan & T2DM, DKD & $\begin{array}{l}\text { Uncontrolled } \\
\text { (single-arm) } \\
\text { study }\end{array}$ & $\begin{array}{l}\text { 8-64 mg } \\
\text { candesartan } \\
\text { ( } n=11) \text {, no control } \\
\text { group }\end{array}$ & 12 weeks & Urinary-CML (ELISA) & $\begin{array}{l}\text { Urinary-CML } \downarrow \text { after candesartan } \\
\text { treatment }\end{array}$ \\
\hline 104 & Valsartan & T2DM & $\begin{array}{l}\text { Uncontrolled } \\
\text { (single-arm) } \\
\text { study }\end{array}$ & $\begin{array}{l}40-80 \text { mg valsartan } \\
(n=15), \text { no control } \\
\text { group }\end{array}$ & $\begin{array}{l}12 \\
\text { months }\end{array}$ & Serum-AGEs (ELISA) & Serum-AGEs $\downarrow$ after valsartan treatment \\
\hline 105 & Valsartan & T2DM, MA & $\begin{array}{l}\text { Uncontrolled } \\
\text { (single-arm) } \\
\text { study }\end{array}$ & $\begin{array}{l}80-160 \mathrm{mg} \\
\text { valsartan }(n=12) \text {, } \\
\text { no control group }\end{array}$ & 6 months & $\begin{array}{l}\text { Plasma- and Urinary-CML } \\
\text { (ELISA) and pentosidine } \\
\text { (HPLC) }\end{array}$ & $\begin{array}{l}\text { Plasma-CML and pentosidine } \downarrow \text { (n.s.) } \\
\text { and Urinary-CML and pentosidine } \downarrow \\
\text { after valsartan treatment }\end{array}$ \\
\hline 106 & Valsartan & T2DM & $\begin{array}{l}\text { Uncontrolled } \\
\text { (single-arm) } \\
\text { study }\end{array}$ & $\begin{array}{l}40 \text { mg valsartan } \\
(n=15), \text { no control } \\
\text { group }\end{array}$ & 6 months & Serum-AGEs (ELISA) & Serum-AGEs $\downarrow$ after valsartan treatment \\
\hline
\end{tabular}


Table A2.3 (continued)

\begin{tabular}{|c|c|c|c|c|c|c|c|}
\hline Ref. & Therapy & $\begin{array}{l}\text { Patient } \\
\text { characteristics }\end{array}$ & Design & $\begin{array}{l}\text { Treatment arms } \\
(n)\end{array}$ & $\begin{array}{l}\text { Treatment } \\
\text { duration }\end{array}$ & Glycation-related outcome & Results \\
\hline 108 & Ramipril & $\mathrm{ND}, \mathrm{CKD}$ & $\begin{array}{l}\text { Open-label } \\
\text { nonrandomized } \\
\text { controlled trial }\end{array}$ & $\begin{array}{l}2.5-5.0 \text { mg ramipril } \\
(n=12) \text {, other BP- } \\
\text { lowering drugs } \\
(n=7)\end{array}$ & 2 months & $\begin{array}{l}\text { Plasma-AGEs (fluorescence) } \\
\text { and CML (ELISA) }\end{array}$ & $\begin{array}{l}\text { AGE-fluorescence } \downarrow \text { in ramipril vs. other } \\
\text { BP-lowering drugs group; no treatment } \\
\text { effects on Plasma-CML }\end{array}$ \\
\hline 98 & Amlodipine & $\begin{array}{l}\text { T2DM, } \\
\text { nephropathy }\end{array}$ & $\begin{array}{l}\text { Double-blind } \\
\text { RCT }\end{array}$ & $\begin{array}{l}10 \mathrm{mg} \text { amlodipine } \\
(n=61), \text { placebo } \\
(n=70)\end{array}$ & 2 years & $\begin{array}{l}\text { Serum-CML (ELISA) and } \\
\text { pentosidine (HPLC) }\end{array}$ & No treatment effects \\
\hline 109 & $\begin{array}{l}\text { Azelnidipine } \\
\text { Amlodipine }\end{array}$ & $\mathrm{ND}, \mathrm{CKD}$ & Open-label RCT & $\begin{array}{l}16 \mathrm{mg} \text { azelnidipine } \\
(n=15) ; 5 \mathrm{mg} \\
\text { amlodipine }(n=15)\end{array}$ & 6 months & Serum-AGEs (ELISA) & $\begin{array}{l}\text { Serum-AGEs } \downarrow \text { in azelnidipine vs. } \\
\text { amlodipine group }\end{array}$ \\
\hline
\end{tabular}

$C K D$, chronic kidney disease; $D K D$, diabetic kidney disease; $F L$, fructoselysine; $G-H 1$, glyoxal-hydroimidazolone; GOLD, glyoxal-derived lysine dimmer; $H D$, hemodialysis; $M A$, microalbuminuria; $M G-H 1$, methylglyoxal-hydroimidazolone; $M O L D$, methylglyoxal-derived lysine dimmer; n.s., not significant. 
Table A2.4 Potential AGE-inhibiting effects of lipid-lowering medication

\begin{tabular}{|c|c|c|c|c|c|c|c|}
\hline Ref. & Therapy & $\begin{array}{l}\text { Patient } \\
\text { characteristics }\end{array}$ & Design & Treatment arms $(n)$ & $\begin{array}{l}\text { Treatment } \\
\text { duration }\end{array}$ & Glycation-related outcome & Results \\
\hline 111 & Atorvastatin & T2DM, HC & Double-blind RCT & $\begin{array}{l}10-20 \text { mg atorvastatin } \\
(n=39, \text { placebo }(n=41)\end{array}$ & 6 months & AGEs (ELISA) & No treatment effects \\
\hline 110 & Cerivastatin & $\begin{array}{l}\text { IGM or T2DM, } \\
\text { hyperlipidemia }\end{array}$ & Double-blind RCT & $\begin{array}{l}0.4 \mathrm{mg} \text { cerivastatin } \\
(n=34), \text { placebo }(n=35)\end{array}$ & 12 weeks & Serum-CML (ELISA) & $\begin{array}{l}\text { Serum-CML } \downarrow \text { in } \\
\text { cerivastatin vs. placebc } \\
\text { group }\end{array}$ \\
\hline 112 & Simvastatin & $\begin{array}{l}\text { T2DM, asymptomatic } \\
\text { carotid artery } \\
\text { stenosis }(>70 \%)\end{array}$ & Open-label RCT & $\begin{array}{l}40 \text { mg simvastatin }+ \\
\text { AHA step } 1 \text { diet } \\
\text { ( } n=35) \text {, AHA step } 1 \\
\text { diet only }(n=35)\end{array}$ & 4 months & $\begin{array}{l}\text { Plaque expression of AGEs, } \\
\text { RAGE } \\
\text { (immunohistochemistry) }\end{array}$ & $\begin{array}{l}\text { Plaque expression of } \\
\text { AGEs, RAGE } \downarrow \text { in } \\
\text { simvastatin }+ \text { diet vs. } \\
\text { diet only group }\end{array}$ \\
\hline 113 & Atorvastatin & $\mathrm{T} 2 \mathrm{DM}, \mathrm{HC}$ & $\begin{array}{l}\text { Open-label } \\
\text { nonrandomized } \\
\text { controlled trial }\end{array}$ & $\begin{array}{l}10 \mathrm{mg} \text { atorvastatin } \\
(n=16), \text { energy- } \\
\text { restricted diet }(n=9)\end{array}$ & 4 weeks & Serum-AGEs (ELISA) & $\begin{array}{l}\text { Serum-AGEs } \downarrow \text { in } \\
\text { atorvastatin vs. diet } \\
\text { only group }\end{array}$ \\
\hline 114 & Atorvastatin & NASH, dyslipidemia & $\begin{array}{l}\text { Uncontrolled } \\
\text { (single-arm) study }\end{array}$ & $\begin{array}{l}10 \text { mg atorvastatin }+ \\
\text { energy-restricted diet } \\
\text { ( } n=43) \text {, no control } \\
\text { group }\end{array}$ & 12 months & Serum-AGEs (ELISA) & $\begin{array}{l}\text { Serum-AGEs } \downarrow \text { after } \\
\text { atorvastatin }+ \text { diet } \\
\text { treatment }\end{array}$ \\
\hline
\end{tabular}

$A H A$, American Heart Association; $H C$, hypercholesterolemia; IGM, impaired glucose metabolism; NASH, non-alcoholic steatohepatitis. 
Table A2.5 Potential AGE-inhibiting effects of medication against rheumatic diseases

\begin{tabular}{|c|c|c|c|c|c|c|c|}
\hline Ref. & Therapy & $\begin{array}{l}\text { Patient } \\
\text { characteristics }\end{array}$ & Design & Treatment arms $(n)$ & $\begin{array}{l}\text { Treatment } \\
\text { duration }\end{array}$ & $\begin{array}{l}\text { Glycation-related } \\
\text { outcome }\end{array}$ & Results \\
\hline 115 & Infliximab & RA & $\begin{array}{l}\text { Uncontrolled } \\
\text { (single-arm) } \\
\text { study }\end{array}$ & $\begin{array}{l}3 \mathrm{mg} / \mathrm{kg} \text { infliximab at baseline, week } 2 \\
\text { and week } 6 \text {, then every } 8 \text { weeks } \\
(n=16), \text { no control group }\end{array}$ & 54 weeks & $\begin{array}{l}\text { Serum- and } \\
\text { Urinary- } \\
\text { pentosidine } \\
\text { (HPLC) }\end{array}$ & $\begin{array}{l}\text { Serum- and Urinary- } \\
\text { pentosidine } \downarrow \text { after } \\
\text { infliximab treatment }\end{array}$ \\
\hline 116 & Etanercept & RA & $\begin{array}{l}\text { Uncontrolled } \\
\text { (single-arm) } \\
\text { study }\end{array}$ & $\begin{array}{l}25 \mathrm{mg} \text { etanercept once or twice/week } \\
(n=22) \text {, no control group }\end{array}$ & 6 months & $\begin{array}{l}\text { Serum- and } \\
\text { Urinary- } \\
\text { pentosidine } \\
\text { (HPLC) }\end{array}$ & $\begin{array}{l}\text { Serum- and Urinary- } \\
\text { pentosidine } \downarrow \text { after } \\
\text { etanercept treatment }\end{array}$ \\
\hline 117 & Methotrexate & RA & $\begin{array}{l}\text { Uncontrolled } \\
\text { (single-arm) } \\
\text { study }\end{array}$ & $\begin{array}{l}\text { 4-8 mg methotrexate/week }(n=19) \text {, no } \\
\text { control group }\end{array}$ & 6 months & $\begin{array}{l}\text { Serum- and } \\
\text { Urinary- } \\
\text { pentosidine } \\
\text { (HPLC) }\end{array}$ & $\begin{array}{l}\text { Serum- and Urinary- } \\
\text { pentosidine } \downarrow \text { after } \\
\text { methotrexate treatment }\end{array}$ \\
\hline
\end{tabular}

$R A$, rheumatoid arthritis. 
Table A2.6 Potential AGE-inhibiting effects of weight-reduction therapies

\begin{tabular}{|c|c|c|c|c|c|c|c|}
\hline Ref. & Therapy & $\begin{array}{l}\text { Patient } \\
\text { characteristics }\end{array}$ & Design & Treatment arms $(n)$ & $\begin{array}{l}\text { Treatment } \\
\text { duration }\end{array}$ & $\begin{array}{l}\text { Glycation-related } \\
\text { outcome }\end{array}$ & Results \\
\hline 123 & Orlistat & $\begin{array}{l}\text { T2DM or healthy } \\
\text { women }\end{array}$ & $\begin{array}{l}\text { Open-label } \\
\text { nonrandomised } \\
\text { crossover study }\end{array}$ & $\begin{array}{l}240 \text { mg orlistat [T2DM }(n=10) \\
\text { or healthy controls }(n=10)] \text {, } \\
\text { control group [T2DM }(n=10) \\
\text { or healthy controls }(n=10)] \text {, } \\
\text { crossover design }\end{array}$ & 1 dose & $\begin{array}{l}\text { Serum-AGEs } \\
\text { (ELISA) }\end{array}$ & $\begin{array}{l}\text { Increase in Serum-AGEs after } \\
\text { high-AGE meal in control group } \\
\text { blunted in orlistat group in } \\
\text { healthy controls; no treatment } \\
\text { effects in T2DM }\end{array}$ \\
\hline 122 & Orlistat & $\begin{array}{l}\text { PCOS or healthy } \\
\text { women }\end{array}$ & $\begin{array}{l}\text { Open-label } \\
\text { nonrandomised } \\
\text { crossover study }\end{array}$ & $\begin{array}{l}240 \text { mg orlistat }(n=36), \\
\text { control group }(n=36), \\
\text { crossover design }\end{array}$ & 1 dose & $\begin{array}{l}\text { Serum-AGEs } \\
\text { (ELISA) }\end{array}$ & $\begin{array}{l}\text { Increase in Serum-AGEs after } \\
\text { high-AGE meal in control group } \\
\text { blunted in orlistat group }\end{array}$ \\
\hline 118 & $\begin{array}{l}\text { Orlistat plus } \\
\text { energy- } \\
\text { restricted } \\
\text { diet }\end{array}$ & $\begin{array}{l}\text { PCOS or healthy } \\
\text { obese women }\end{array}$ & Uncontrolled study & $\begin{array}{l}360 \mathrm{mg} \text { orlistat }+600 \mathrm{kcal} \text { diet } \\
(n=18) \text {, no control group }\end{array}$ & 6 months & $\begin{array}{l}\text { Serum-AGEs } \\
\text { (ELISA) }\end{array}$ & $\begin{array}{l}\text { Serum-AGEs } \downarrow \text { after orlistat + } \\
\text { diet }\end{array}$ \\
\hline 119 & $\begin{array}{l}\text { Energy- } \\
\text { restricted } \\
\text { diet }\end{array}$ & $\begin{array}{l}\text { Overweight or } \\
\text { obese }\end{array}$ & $\begin{array}{l}\text { Uncontrolled } \\
\text { (single-arm) study }\end{array}$ & $\begin{array}{l}1200 \text { kcal diet }+ \text { diet } \\
\text { education }(n=37) \text {, no control } \\
\text { group }\end{array}$ & 2 months & $\begin{array}{l}\text { Serum-AGEs } \\
\text { (fluorescence) }\end{array}$ & Serum-AGEs $\downarrow$ after diet \\
\hline 120 & $\begin{array}{l}\text { Energy- } \\
\text { restricted } \\
\text { diet }\end{array}$ & RA & $\begin{array}{l}\text { Uncontrolled } \\
\text { (single-arm) study }\end{array}$ & $\begin{array}{l}\text { RA }+720-1085 \text { kcal diet } \\
(n=10), \text { no control group }\end{array}$ & 54 days & $\begin{array}{l}\text { Urinary- } \\
\text { pentosidine } \\
\text { (HPLC) }\end{array}$ & Urinary-pentosidine $\downarrow$ after diet \\
\hline 121 & $\begin{array}{l}\text { Exercise } \\
\text { program }\end{array}$ & Healthy women & $\begin{array}{l}\text { Open-label } \\
\text { nonrandomised } \\
\text { controlled study }\end{array}$ & $\begin{array}{l}90 \mathrm{~min} / \text { wk exercise program } \\
+ \text { exercise education }(n=17), \\
\text { control group }(n=12)\end{array}$ & 12 weeks & $\begin{array}{l}\text { Serum-CML and } \\
\text { pentosidine } \\
\text { (ELISA) }\end{array}$ & $\begin{array}{l}\text { Serum-CML } \downarrow \text { in exercise vs. } \\
\text { control group; no treatment } \\
\text { effects on Serum-pentosidine }\end{array}$ \\
\hline
\end{tabular}

$P C O S$, polycystic ovary syndrome; $R A$, rheumatoid arthritis. 
Table A2.7 Potential AGE-inhibiting effects of AGE-restricted diets

\begin{tabular}{|c|c|c|c|c|c|c|c|}
\hline Ref. & Therapy & $\begin{array}{l}\text { Patient } \\
\text { characteristics }\end{array}$ & Design & Treatment arms $(n)$ & $\begin{array}{l}\text { Treatment } \\
\text { duration }\end{array}$ & Glycation-related outcome & Results \\
\hline 125 & $\begin{array}{l}\text { AGE- } \\
\text { restricted } \\
\text { diet }\end{array}$ & T2DM & $\begin{array}{l}\text { Open- } \\
\text { label } \\
\text { RCT }\end{array}$ & $\begin{array}{l}\text { 1: AGE-restricted diet (<10 AGE } \\
\text { Eq/day; } n=12 \text { ), isocaloric standard } \\
\operatorname{diet~(>20~AGE~Eq/day;~} n=6)\end{array}$ & 4 months & $\begin{array}{l}\text { Serum-CML and MG } \\
\text { derivatives (ELISA), PMNC- } \\
\text { AGER1 and RAGE } \\
\text { expression }\end{array}$ & $\begin{array}{l}\text { Serum-CML and MG derivatives } \downarrow \\
\text { and PMNC-AGER1 and RAGE } \uparrow \text { in } \\
\text { AGE-restricted vs. standard diet } \\
\text { group }\end{array}$ \\
\hline 127 & $\begin{array}{l}\text { AGE- } \\
\text { restricted } \\
\text { diet }\end{array}$ & $\begin{array}{l}\text { Healthy } \\
\text { individuals }\end{array}$ & $\begin{array}{l}\text { Open- } \\
\text { label } \\
\text { RCT }\end{array}$ & $\begin{array}{l}\text { AGE-restricted diet ( }<10 \text { AGE } \\
\text { Eq/day; } n=30) \text {, isocaloric usual } \\
\operatorname{diet}(n=10)\end{array}$ & 4 months & $\begin{array}{l}\text { Serum-CML and MG } \\
\text { derivatives (ELISA), PMNC- } \\
\text { AGER1 and RAGE } \\
\text { expression }\end{array}$ & $\begin{array}{l}\text { Serum-CML and MG derivatives, } \\
\text { PMNC-AGER1 and RAGE } \downarrow \text { in AGE- } \\
\text { restricted vs. standard diet group }\end{array}$ \\
\hline 124 & $\begin{array}{l}\text { AGE- } \\
\text { restricted } \\
\text { diet }\end{array}$ & $\begin{array}{l}\text { T1DM or } \\
\text { T2DM }\end{array}$ & $\begin{array}{l}\text { Open- } \\
\text { label } \\
\text { RCT }\end{array}$ & $\begin{array}{l}\text { AGE-restricted diet (5-fold lower } \\
\text { AGE; } n=13) \text {, isocaloric standard } \\
\text { diet }(n=11)\end{array}$ & 6 weeks & $\begin{array}{l}\text { Serum-CML and MG } \\
\text { derivatives (ELISA), LDL- } \\
\text { AGEs (ELISA) }\end{array}$ & $\begin{array}{l}\text { Serum-CML and MG derivatives, } \\
\text { LDL-AGEs } \downarrow \text { in AGE-restricted vs. } \\
\text { standard diet group }\end{array}$ \\
\hline 126 & $\begin{array}{l}\text { AGE- } \\
\text { restricted } \\
\text { diet }\end{array}$ & PD patients & $\begin{array}{l}\text { Open- } \\
\text { label } \\
\text { RCT }\end{array}$ & $\begin{array}{l}\text { AGE-restricted diet }(n=9) \text {; } \\
\text { standard diet }(n=9)\end{array}$ & 4 weeks & $\begin{array}{l}\text { Serum- and Urinary-CML } \\
\text { and MG derivatives (ELISA), } \\
\text { LDL-CML (ELISA) }\end{array}$ & $\begin{array}{l}\text { Urinary- (n.s.) and Serum-CML and } \\
\text { MG derivatives and LDL-CML } \downarrow \text { in } \\
\text { AGE-restricted vs. standard diet } \\
\text { group }\end{array}$ \\
\hline
\end{tabular}

$E q$, equivalents; $P D$, peritoneal dialysis; $P M N C$, peripheral blood mononuclear cells. 
Table A2.8 Potential AGE-inhibiting effects of 'other' therapies

\begin{tabular}{|c|c|c|c|c|c|c|c|}
\hline Ref. & Therapy & $\begin{array}{l}\text { Patient } \\
\text { characteristics }\end{array}$ & Design & Treatment arms $(n)$ & $\begin{array}{l}\text { Treatment } \\
\text { duration }\end{array}$ & Glycation-related outcome & Results \\
\hline 128 & Oestrogen & $\begin{array}{l}\text { Post-menopausal } \\
\text { women, SUI }\end{array}$ & Double-blind RCT & $\begin{array}{l}2 \text { mg oestradiol valerate } \\
(n=26), \text { placebo }(n=23)\end{array}$ & 6 months & $\begin{array}{l}\text { Vaginal epithelium- } \\
\text { pentosidine and NFC-1 } \\
\text { (HPLC) }\end{array}$ & $\begin{array}{l}\text { Vaginal epithelium- } \\
\text { pentosidine (n.s.) and } \\
\text { NFC- } 1 \downarrow \text { in oestrogen vs. } \\
\text { placebo group }\end{array}$ \\
\hline 130 & $\begin{array}{l}\text { Anti-H pylori } \\
\text { treatment }\end{array}$ & $\begin{array}{l}\text { Anemia, } H \text { pylori } \\
\text { infection }\end{array}$ & Open-label RCT & $\begin{array}{l}\text { Anti- } H \text { pylori therapy }+ \\
600 \mathrm{mg} \text { ferrous sulphate } \\
(n=11), \text { ferrous sulphate } \\
\text { only }(n=11)\end{array}$ & 1 month & $\begin{array}{l}\text { Plasma-fructosamine ( } \mathrm{p} \text { - } \\
\text { indonitrotetrazolium violet } \\
\text { kinetic method) }\end{array}$ & No treatment effects \\
\hline 129 & $\begin{array}{l}\text { Iron } \\
\text { supplements }\end{array}$ & ND, anemia & $\begin{array}{l}\text { Uncontrolled } \\
\text { (single-arm) study }\end{array}$ & $\begin{array}{l}600 \mathrm{mg} \text { ferrous sulphate } \\
(n=22), \text { no anemia } \\
\text { control group }\end{array}$ & 1 month & $\begin{array}{l}\text { Serum-fructosamine ( } p \text { - } \\
\text { indonitrotetrazolium violet } \\
\text { kinetic method) }\end{array}$ & $\begin{array}{l}\text { Serum-fructosamine } \downarrow \\
\text { after iron treatment }\end{array}$ \\
\hline 131 & DHEA & T2DM & RCT & $\begin{array}{l}50 \mathrm{mg} \text { DHEA }(n=10) \text {, } \\
\text { placebo }(n=10)\end{array}$ & 12 weeks & $\begin{array}{l}\text { Plasma-pentosidine (LC- } \\
\text { tandem MS) }\end{array}$ & $\begin{array}{l}\text { Plasma-pentosidine } \downarrow \\
\text { after DHEA treatment; no } \\
\text { changes in placebo } \\
\text { group* }\end{array}$ \\
\hline 132 & $\begin{array}{l}\text { Aspirin } \\
\text { Basic amino } \\
\text { acids }\end{array}$ & T2DM & Double-blind RCT & $\begin{array}{l}100 \mathrm{mg} \text { aspirin }(n=7), 2 \\
\mathrm{~g} \mathrm{~L}-\text { arginine }+0.5 \mathrm{~g} \mathrm{~L}- \\
\text { lysine }(n=6), \text { placebo } \\
(n=6)\end{array}$ & 1 year & $\begin{array}{l}\text { Skin tissue-pentosidine } \\
\text { (HPLC) }\end{array}$ & $\begin{array}{l}\text { Skin tissue-pentosidine } \downarrow \\
\text { after aspirin treatment; } \\
\text { no changes in other } \\
\text { groups* }\end{array}$ \\
\hline 133 & $\begin{array}{l}\text { Danggui Sini } \\
\text { Cilostazol }\end{array}$ & $\begin{array}{l}\text { Diabetic foot } \\
\text { ulcer }\end{array}$ & Open-label RCT & $\begin{array}{l}\text { Danggui Sini Decoction } \\
(n=36), 100 \mathrm{mg} \\
\text { cilostazol }(n=36)\end{array}$ & 6 weeks & Plasma-AGEs (ELISA) & $\begin{array}{l}\text { Plasma-AGEs } \downarrow \text { in Danggui } \\
\text { Sini group vs. cilostazol } \\
\text { group }\end{array}$ \\
\hline 134 & Sevelamer & HD patients & Open-label RCT & $\begin{array}{l}9 \text { g sevelamer }+1.5 \mathrm{~g} \\
\text { calcium carbonate } \\
(n=91), \text { calcium } \\
\text { carbonate only }(n=91)\end{array}$ & 1 year & Plasma-pentosidine (HPLC) & $\begin{array}{l}\text { Plasma-pentosidine } \downarrow \text { in } \\
\text { sevelamer vs. control } \\
\text { group }\end{array}$ \\
\hline $135 *$ & Vitamin $\mathrm{E}$ & $\mathrm{DM}$ & $\begin{array}{l}\text { Double-blind } \\
\text { randomised } \\
\text { crossover study }\end{array}$ & $\begin{array}{l}1200 \text { mg vitamin } \mathrm{E} \\
(n=53), \text { placebo }(n=53), \\
\text { crossover design }\end{array}$ & 2 months & Serum-fructosamine & No treatment effects \\
\hline
\end{tabular}


Table A2.8 (continued)

\begin{tabular}{|c|c|c|c|c|c|c|c|}
\hline Ref. & Therapy & $\begin{array}{l}\text { Patient } \\
\text { characteristics }\end{array}$ & Design & Treatment arms $(n)$ & $\begin{array}{l}\text { Treatment } \\
\text { duration }\end{array}$ & Glycation-related outcome & Results \\
\hline 136 & Vitamin $\mathrm{E}$ & HD patients & $\begin{array}{l}\text { Double- } \\
\text { blind RCT }\end{array}$ & $\begin{array}{l}800 \text { IU vitamin E }(n=14), \text { placebo } \\
(n=13)\end{array}$ & 6 months & Plasma-pentosidine (HPLC) & $\begin{array}{l}\text { No } \\
\text { treatment } \\
\text { effects }\end{array}$ \\
\hline 137 & Vitamin $\mathrm{E}$ & T2DM & $\begin{array}{l}\text { Double- } \\
\text { blind RCT }\end{array}$ & $\begin{array}{l}1,600 \text { IU vitamin E }(n=10) \text {, } \\
\text { placebo }(n=11)\end{array}$ & 10 weeks & $\begin{array}{l}\text { Total Plasma-protein glycation (HPLC), } \\
\text { glycated albumin (immunoturbidimetry), } \\
\text { glycated LDL }\end{array}$ & $\begin{array}{l}\text { No } \\
\text { treatment } \\
\text { effects }\end{array}$ \\
\hline 138 & $\begin{array}{l}\text { Propionyl L- } \\
\text { carnitine } \\
\text { (PLC) } \\
\text { Sildenafil }\end{array}$ & $\begin{array}{l}\text { T2DM, erectile } \\
\text { dysfunction }\end{array}$ & $\begin{array}{l}\text { Double- } \\
\text { blind RCT }\end{array}$ & $\begin{array}{l}2 \text { g PLC }+100 \text { mg sildenafil twice } \\
\text { weekly }(n=8) \text {, PLC only }(n=8) \\
\text { sildenafil only }(n=8) \text {, placebo } \\
(n=8)\end{array}$ & 12 weeks & Serum-AGEs (ELISA) & $\begin{array}{l}\text { No } \\
\text { treatment } \\
\text { effects }\end{array}$ \\
\hline
\end{tabular}

$D H E A$, dehydroepiandrosterone; $N F C$, non-fluorescent compound 1; SRP, scaling and root planning; SUI, stress urinary incontinence.

* Differences between groups in change in pentosidine levels after treatment were not provided. 


\section{CHAPTER 3}

IMPROYED ŚLYCAEMIC CONTROL INDUCED BY BOTH METFORMIN AMD REPAŚLINIDE IS ASSOCIATED HITH A REDUCTION IN BLOOD LEVELS OF 3-DEOXYSLLUCOSONE IN NON-OBESE PATIENTS WITH TYPE 2 DIABETES

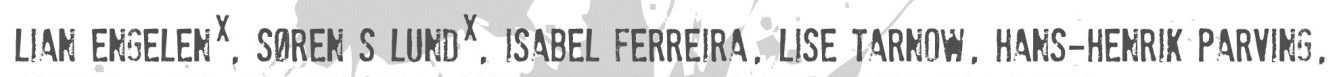
JRRSEEM SRAM, KAJ WIITHER, OLUF PEDERSEK, TOM TEERLLIKK, ROB BARTO,

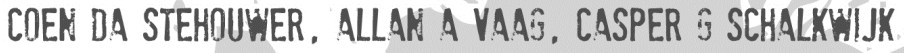

${ }^{x}$ EQUal COATRBUTtON.

EUR J ENDOCRINOL. 2011 MAR:164(3):371-9 


\section{ABSTRACT}

\section{Background}

Metformin has been reported to reduce alpha-dicarbonyls, which are known to contribute to diabetic complications. It is unclear whether this is due to direct quenching of alphadicarbonyls or to an improvement in glycaemic control. We therefore compared the effects of metformin vs. repaglinide, an anti-hyperglycaemic agent with an insulinsecreting mechanism, on the levels of the alpha-dicarbonyl 3-deoxyglucosone (3DG).

\section{Methods}

We conducted a single-centre, double-blind, double-dummy, crossover study involving 96 non-obese patients with type 2 diabetes. After a one-month run-in on diet-only treatment, patients were randomized to either repaglinide (6 $\mathrm{mg}$ daily) followed by metformin ( $2 \mathrm{~g}$ daily) or vice versa each during four months with a 1-month washout between interventions.

\section{Results}

3DG levels decreased after both metformin [-19.3\% (95\% $\mathrm{Cl}:-23.5 ;-14.8)]$ and repaglinide $[-20.8 \%(-24.9 ;-16.3)]$ treatments, but no difference was found between treatments [1.8\% $(-3.8 ; 7.8)]$. Regardless of treatment, changes in glycaemic variables were associated with changes in 3DG. Specifically, 3DG decreased by $22.7 \%(19.0 ; 26.5)$ per SD decrease in fasting plasma glucose (PG), by $20.0 \%(16.2 ; 23.9)$ per SD decrease in 7-point MPG, by $22.5 \%(18.6 ; 26.6)$ per SD decrease in AUC-PG, by $17.2 \%(13.8 ; 20.6)$ per SD decrease in $\mathrm{HbA}_{1 \mathrm{c}}$ and by $10.9 \%(6.4 ; 15.5)$ per SD decrease in Amadori albumin. In addition, decreases in 3DG were associated with decreases in advanced glycation endproducts and endothelial markers.

\section{Conclusion}

Improved glycaemic control induced by both metformin and repaglinide is associated with a reduction in 3DG levels in non-obese individuals with type 2 diabetes. This may constitute a shared metabolic pathway through which both treatments have a beneficial impact on the cardiovascular risk. 


\section{INTRODUCTION}

The mechanisms underlying the development of vascular complications in diabetes are not completely understood. A potential mechanism by which hyperglycaemia and its immediate biochemical sequelae induce vascular complications involves the increased formation of advanced glycation endproducts (AGEs). ${ }^{1}$ The deleterious effects of AGEs are attributed to direct modification of extracellular matrix proteins, resulting in collagen cross-links, which lead to increased vascular stiffness. ${ }^{1}$ In addition, binding of AGEs to a variety of cell-surface receptors, such as the receptor for advanced glycation endproducts (RAGE), triggers signal transduction pathways and the production of reactive oxygen species (ROS), resulting in proinflammatory cellular responses. ${ }^{2}$ Given these deleterious effects of AGEs, several potential inhibitors (e.g. aminoguanidine, pyridoxamine, and cross-link breaker ALT-711) have been developed to reduce the clinical impact of AGEs. ${ }^{3}$

Although the formation of AGEs is complex, it is well-established that alphadicarbonyl compounds such as 3-deoxyglucosone (3DG) and methylglyoxal are reactive intermediates in the formation of AGEs. ${ }^{4}$ Levels of 3DG have been shown to be elevated in patients with diabetes, ${ }^{5,6}$ particularly in those with diabetic microangiopathy, such as retinopathy, ${ }^{5}$ neuropathy ${ }^{5}$ and nephropathy. ${ }^{5,7}$

Aminoguanidine, which is a small hydrazine-like molecule, inhibits the formation of AGEs by interaction with and quenching of dicarbonyl compounds. ${ }^{8}$ Metformin, an antihyperglycaemic agent which enhances insulin sensitivity and lowers hepatic glucose output may also have anti-glycation properties, with a working mechanism similar to aminoguanidine due to a similarity in structure between both agents. ${ }^{9-11}$ The observation of reduced levels of methylglyoxal in patients using metformin as compared with controls support this view. ${ }^{9}$ Based on this additional effect, the beneficial impact of metformin on cardiovascular complications of type 2 diabetes, as for instance reported in the UKPDS study, ${ }^{12}$ could be, at least in part, ascribed to its specific effects on detoxifying dicarbonyl compounds thereby preventing AGE accumulation. However, a reduction in dicarbonyls following treatment with metformin may also derive from an improvement in glycaemic control.

In view of these considerations, and in order to disentangle those two possible operating mechanisms, we have investigated, in a single-centre, double-masked, doubledummy, crossover study in non-obese patients with type 2 diabetes [initially investigating the hypothesis of equivalent antihyperglycaemic effects of metformin and repaglinide; ClinicalTrials.gov Identifier: NCT00118950 ${ }^{13-15}$ ]: 1) the effects of metformin vs. repaglinide - an anti-hyperglycaemic agent with a different working mechanism (i.e. stimulating insulin secretion) - on the levels of 3DG; 2 ) the associations of treatment-induced changes 
in glycaemic variables (i.e. fasting plasma glucose (PG), 7-point mean PG, AUC-PG, HbA and Amadori albumin) with changes in 3DG levels; and 3) associations between changes in 3DG levels and changes in biomarkers of advanced glycation, endothelial dysfunction and low-grade inflammation.

\section{METHODS}

\section{STUDY DESIGN}

In 2001-2003, we conducted a single-centre, randomized, double-blind, double-dummy, crossover study with 96 non-obese (body mass index (BMI) $\leq 27 \mathrm{~kg} / \mathrm{m}^{2}$ ) insulin-naive individuals with type 2 diabetes as described in detail elsewhere. ${ }^{13-15}$ Briefly, we included 96 subjects who had type 2 diabetes diagnosed when 40 years of age or older, had no history of ketonuria or diabetic ketoacidosis and, at enrolment, presented with a fasting serum C-peptide $\geq 300 \mathrm{pmol} / \mathrm{L}$ or a non-fasting or glucagon-stimulated C-peptide $\geq 600$ pmol/L. After enrolment, patients stopped intake of oral hypoglycaemic agents and underwent a one-month run-in period on diet-only treatment. After the run-in period, patients were randomised to receive either $2 \mathrm{mg}$ repaglinide three times daily plus placebo metformin followed by $1 \mathrm{~g}$ metformin twice daily plus placebo repaglinide, or vice versa, each treatment for a period of four months with a one-month washout period between interventions.

Out of the 96 included patients, 93 and 89 patients initiated a treatment period with metformin and repaglinide, respectively. Throughout the course of treatments 20 patients (21\%) dropped out, leaving 76 patients (79\%) who completed the whole trial; 83 and 82 patients completed a treatment period with metformin or repaglinide, respectively. Ten (11\%) patients dropped out during metformin treatment, 7 (8\%) during repaglinide treatment and $3(3 \%)$ during the washout period; 1 after repaglinide and 2 after metformin treatment (Figure 3.1). ${ }^{14}$

Results regarding the effects of treatments on trial's primary endpoint - $\mathrm{HbA}_{1 c}$, and secondary endpoints such as postprandial glucose and lipid metabolism, as well as markers of inflammation and endothelial dysfunction, have been reported previously. ${ }^{13-15}$ The present study focuses specifically on the effects of treatments on blood levels of 3DG, which due to the complexity of its assessment only became available recently. Measures to ensure proper blood sampling to enable future assessment of 3DG levels were planned prior to and undertaken during the trial (see also below).

The study was conducted in accordance with the Declaration of Helsinki and was approved by the Ethics Committee of Copenhagen County, Denmark. 


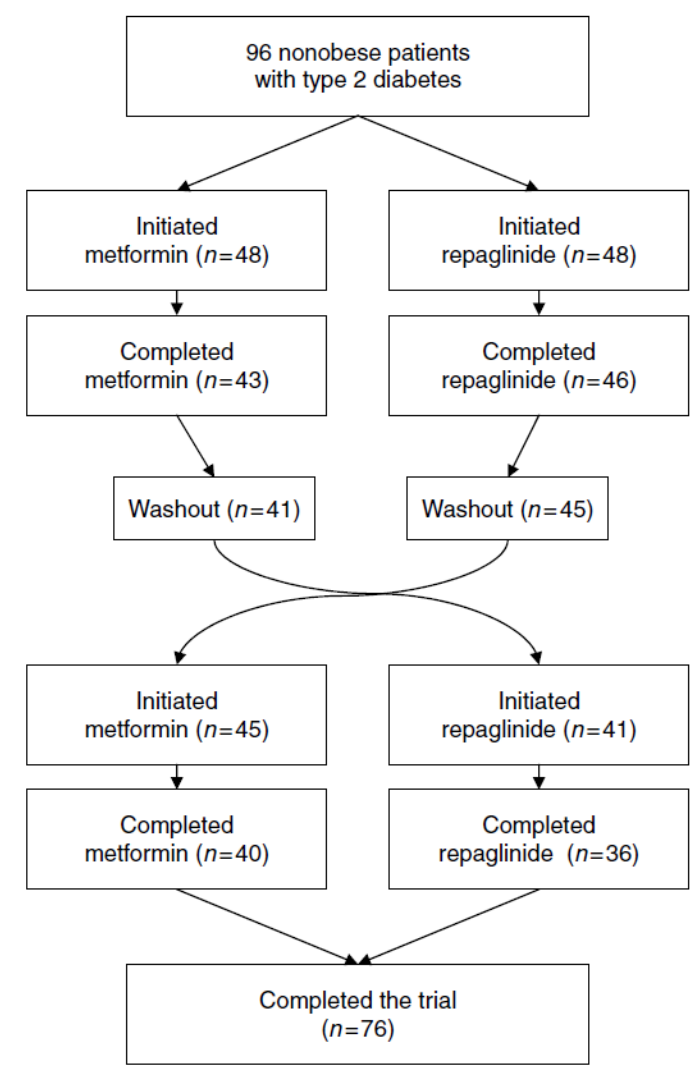

Figure 3.1 Study design and flow chart of the 96 randomised patients

\section{BLOOD SAMPLING}

Patients were instructed to obtain seven-point home-monitored plasma glucose measurements (pre-prandial, 90 minutes post-prandial and pre-bedtime) two days per week during treatment periods, which were used to calculate the mean plasma glucose levels (7-point MPG).

Patients were further investigated at the Steno Diabetes Center (Gentofte, Denmark) where blood samples were taken during the last week before the patient entered a treatment period and on the last day of each treatment period. Blood samples were drawn with minimal venous occlusion between 8 and 9 AM after a 10-hour overnight fast and 25 minutes of supine rest. Additionally, during a standardized 0-6 hour meal-test, blood was withdrawn at $t=0,90,180,270$ and $360 \mathrm{~min}$, and used to calculate the area under the curve for plasma glucose (AUC-PG) by the trapezoidal rule (i.e. $1.5 \mathrm{~h} *(0.5 *$ 
concentration $_{t=0 \mathrm{~h}}+$ concentration $_{t=1.5 \mathrm{~h}}+$ concentration $_{t=3.0 \mathrm{~h}}+$ concentration $_{t=4.5 \mathrm{~h}}+0.5 *$ concentration $_{t=6.0 \mathrm{~h}}$ ) in $\mathrm{mmol} / \mathrm{L} *$ hours). ${ }^{13}$

Sodium citrate and EDTA plasma and serum samples were prepared by centrifugation at $2,000 \mathrm{~g}$ for 10 minutes at $20^{\circ} \mathrm{C}$ and immediately frozen hereafter. Samples were stored at $-80^{\circ} \mathrm{C}$ until analyses.

Alpha-dicarbonyls (such as 3DG) react rapidly with arginine and lysine residues in proteins. Therefore, as the measurement of 3DG was anticipated prior to initiating the trial, $0.5 \mathrm{~mL}$ whole blood was collected in specific blood collection tubes to be immediately mixed with $1.0 \mathrm{~mL}$ of $1.2 \mathrm{~mol} / \mathrm{L}$ perchloric acid to precipitate the protein fraction and stored at $-80^{\circ} \mathrm{C}$ until assayed.

\section{BIOCHEMICAL ANALYSES}

3DG was measured using liquid chromatography tandem mass spectrometry (LC-tandem MS). ${ }^{6}$ After thawing the acidified blood samples, the samples were centrifuged ( $5 \mathrm{~min}$ at $20,000 \mathrm{~g}$ ) and $80 \mu \mathrm{L}$ of the supernatant was mixed with $100 \mu \mathrm{L}$ of the internal standard solution ( $1 \mu \mathrm{mol} / \mathrm{L}$ 2,3-pentanedione dissolved in ethanol). After the addition of $20 \mu \mathrm{L}$ of the derivatisation reagent $(10 \mathrm{mmol} / \mathrm{L} \mathrm{2,4-dinitrophenylhydrazine} \mathrm{dissolved} \mathrm{in} 1.2 \mathrm{~mol} / \mathrm{L}$ perchloric acid), the samples were incubated for $16 \mathrm{~h}$ at room temperature. Chromatographic separation was performed on an Xterra MS C18 column (4.6*50 mm, 3.5- $\mu \mathrm{m}$ particle size) (Waters, Milford, MA, USA) using a linear gradient from 40 to $95 \%$ acetonitrile in water over $9 \mathrm{~min}$ at a flow rate of $1 \mathrm{~mL} / \mathrm{min}$. Mass transitions of 521.1 to 431.0 and 521.1 to182.1 for 3DG and 459.1 to182.1 for the internal standard were monitored in negative ion mode. The derivatisation and the chromatographic separation are performed at a low $\mathrm{pH}$, minimizing the chance of 3DG formation from sugars and fructosyl-lysine at this stage. ${ }^{4}$ To test the recovery of 3DG, calibration curves prepared in whole blood (two different samples) were compared to a calibration curve prepared in water. The slopes of the calibration curves in whole blood were not significantly different from the slope of the calibration curve in water. As part of validation of the method, we performed a comparison with a previously published method, based on derivatisation with 1,2-diamino-4,5-dimethoxybenzene (DDB) followed by HPLC analysis with fluorescence detection. ${ }^{16}$ In a comparison of perchloric acid supernatants of 44 whole blood samples, results obtained with both methods were highly correlated ( $r=0.92$; $p<0.001)$. Comparison of both methods by Deming regression showed that intercept and slope of the regression line were not significantly different from zero and one, respectively. Mean \pm SD 3DG concentrations obtained by LC-MS/MS and LC-fluorescence were $188 \pm 72$ and $181 \pm 61 \mathrm{nM}$, respectively. Values obtained by both methods were not significantly different. Intra- and inter-assay coefficients of variation were $<10 \%$. 
Plasma glucose (at study-visits: venous samples; home-monitored: fresh capillary blood samples) was measured with the glucose-oxidase biosensor method (EuroFlash handdevice, LifeScan, Milpitas, CA, USA; calibrated for plasma-glucose with the YSI Model 2300 Glucose Analyser, YSI Inc., Yellow Springs, $\mathrm{OH}$, USA). The coefficient of variation between test-strips was $<10 \%$. $\mathrm{HbA}_{1 \mathrm{c}}$ was measured by ion-exchange HPLC-method traceable to the Diabetes Control and Complication Trial (DCCT) standard (Bio-Rad VARIANT method, BioRad Diagnostics Group, Hercules, CA, USA). Amadori albumin was measured in sodium citrate plasma with ELISA. ${ }^{17}$

$\mathrm{N}^{\varepsilon}$ (1-carboxymethyl)lysine $(\mathrm{CML})$ and $\mathrm{N}^{\varepsilon}(1$-carboxyethyl)lysine $(\mathrm{CEL})$ residues in plasma proteins were measured by stable-isotope dilution tandem $\mathrm{MS}^{18}$

The concentration of pyrraline in the protein-free plasma fraction was determined by UPLC-tandem MS. Because a stable isotope pyrraline internal standard was not commercially available, we have chosen, after extensive validation, $\left[{ }^{13} \mathrm{C}_{9}\right]-3-$ Nitrotyrosine as a non-analog internal standard. A $25 \mu \mathrm{L}$ plasma fraction was mixed thoroughly with $12.5 \mu \mathrm{L}$ internal standard (3.9 $\mu \mathrm{M}\left[{ }^{13} \mathrm{C}_{9}\right]-3$-Nitrotyrosine) and $500 \mu \mathrm{L}$ methanol:acetonitrile (1:3, by volume). Proteins were removed by centrifugation (20 minutes at $14,000 \mathrm{~g}$ ) and the supernatant was evaporated to dryness at $65^{\circ} \mathrm{C}$ under a constant stream of nitrogen. The residue was dissolved in $150 \mu \mathrm{L}$ water. Reversed-phase chromatography was carried out using an Acquity UPLC BEH $\mathrm{C}_{18}$ column $(2.1 \times 50 \mathrm{~mm}, 1.7$ $\mu \mathrm{m}$ particle size, Waters, Milford, MA, USA) with $10 \mathrm{mM}$ formic acid as solvent $A$ and acetonitrile as solvent B. A linear gradient was started at $99 \%$ solvent $A$, which was changed in 1.5 minutes to $95.5 \%$ solvent $A$. After cleaning the column with $45 \%$ solvent $B$ during 4 minutes the column was equilibrated for 2 minutes at the initial conditions. Injection volume was $2 \mu \mathrm{L}$ and column temperature was set at $48^{\circ} \mathrm{C}$. Detection was carried out using a Xevo TQ tandem mass spectrometer (Waters, Milford, MA, USA) in electrospray positive mode. Multiple reaction monitoring transitions were set at 236.10 to 189.15 for $\left[{ }^{13} \mathrm{C}_{9}\right]-3$-nitrotyrosine and at 255.10 to 175.20 for pyrraline. Capillary voltage was set at $0.5 \mathrm{kV}$ and a cone voltage of $14 \mathrm{~V}$ and $10 \mathrm{~V}$ was used for $\left[{ }^{13} \mathrm{C}_{9}\right]$-3-nitrotyrosine and pyrraline respectively. We tested recovery (and thus matrix effects), based on additions of pyrraline standard to different matrices. Mean recovery, tested in three different matrices, was $98.5 \pm 5.3 \%$. Linearity was tested in eleven different matrices by adding known pyrraline concentrations between 0 and $300 \mathrm{nmol} / \mathrm{L}$. Mean response factor was $1.44 \pm 0.11$ and $1.33 \pm 0.06$ in plasma and water, respectively. The intra-assay variation was $3.5 \%$ as determined in a plasma sample $(n=10)$ with a mean pyrraline concentration of $89.8 \mathrm{nmol} / \mathrm{L}$. The inter-assay variation was $4.9 \%$ as determined in a plasma sample $(n=7)$ with a mean pyrraline concentration of $85.2 \mathrm{nM}$. The lower limit of quantification was $19.5 \mathrm{fmol}$ at an injection volume of $5 \mu \mathrm{l}$ (signal to noise ratio $(\mathrm{s} / \mathrm{N})=6$ ).

For the evaluation of endothelial dysfunction, we measured plasma levels of plasminogen activator inhibitor-1 antigen (PAl-1-ag), tissue-type plasminogen activator 
antigen (t-PA-ag; TintElize PAI-1 and Immulyse t-PA, respectively; Biopool, Umeå, Sweden) and von Willebrand factor (vWf) antigen using a highly sensitive immunoassay. ${ }^{19}$ Serum levels of soluble intercellular adhesion molecule-1 (sICAM-1), soluble vascular cell adhesion molecule-1 (sVCAM-1) and soluble E-selectin (sE-selectin) were measured using commercially available ELISA kits (Diaclone, Besançon, France).

For the evaluation of low-grade inflammation, we measured levels of serum Creactive protein (CRP) using a highly sensitive enzyme immunoassay. ${ }^{19}$ Plasma levels of tumour necrosis factor alpha (TNF- $\alpha$ ) and serum levels of interleukin-6 (IL-6) were measured using commercially available ELISA kits (Quantikine High Sensitivity; R\&D Systems, Oxon, UK) and fibrinogen was measured using the Multifibren $U$ reagent (Dade Behring, Marburg, Germany).

\section{STATISTICS}

Outcome variables were evaluated after the run-in period (i.e. before initiating treatment in the first period), referred to as first-period baseline; after the washout period (i.e. before initiating treatment in the second period), referred to as second-period baseline; and on the last day of each treatment period. Treatment effects were estimated as change from first-period baseline.

Comparisons between first- and second-period baseline levels of the metabolic variables examined in the present study were performed with the use of Student's t test. We used a linear mixed model, with subject as a random effect, to compare the treatment effects of metformin vs. repaglinide on changes in 3DG levels. Treatment type (metformin or repaglinide), treatment sequence (metformin followed by repaglinide or vice versa), the period effect and the first-period baseline levels of 3DG were included as fixed effects. The extent to which the potential occurrence of unexpected carry-over effects might have influenced sequence and period effects, was thereby taken into account. Furthermore, analyses with a linear mixed model enabled information from incomplete blocks (i.e. those with drop-outs) to be included (i.e. both within- and between-subject information was included).

Associations of changes in other metabolic variables with changes in 3DG were investigated by adding baseline and changes in glycaemic variables (i.e. FPG, 7-point MPG, AUC-PG, $\mathrm{HbA}_{1 \mathrm{c}}$ and Amadori albumin) to our initial model. Associations between changes in 3DG and changes in biomarkers of advanced glycation (i.e. CML, CEL and pyrraline), endothelial dysfunction (i.e. PAI-1-ag, tPA-ag, vWf, sICAM-1, sVCAM-1 and sE-selectin) and low-grade inflammation (i.e. CRP, TNF- $\alpha$, IL- 6 and fibrinogen) were evaluated in a similar way. Thereby, associations of changes in these variables with changes in 3DG levels were thus adjusted for treatment type and sequence, the period effect and first-period baseline 
levels of both 3DG and the other studied metabolic variable. In order to enable comparison of the strength of these associations, we used standardized [i.e. (subjects' value - population mean) / SD] values of changes in the metabolic variables. Variables with skewed distribution (i.e. 3DG, pyrraline, PAI-1-ag, sICAM-1, sVCAM-1, sE-Selectin, CRP, TNF- $\alpha$ and IL-6) were logarithmically transformed prior to analyses and are presented as median and inter-quartile range (IQR). Treatment effects on 3DG and associations between changes in other metabolic variables and changes in 3DG and between changes in 3DG and changes in biomarkers with skewed distribution are presented as percentages due to required back transformation of the regression coefficients.

All statistical analyses were carried out using the Statistical Package for Social Sciences, version 15.0 (SPSS Inc., Chicago, IL, USA).

\section{RESULTS}

Patients' characteristics at enrolment are shown in Table 3.1. A more detailed description of patients' characteristics (e.g. use of medication) at enrolment as well as of adverse events during the trial have been presented elsewhere. ${ }^{14}$

Table 3.1 Clinical characteristics of the study participants at enrolment

\begin{tabular}{lcc}
\hline & $\begin{array}{c}\text { repaglinide followed } \\
\text { by metformin }(n=48)\end{array}$ & $\begin{array}{c}\text { metformin followed } \\
\text { by repaglinide }(n=48)\end{array}$ \\
\hline Men/women, $n$ & $36 / 12$ & $37 / 11$ \\
Age (years) & $63.3 \pm 8.9$ & $59.5 \pm 9.3$ \\
Known duration of diabetes (years) & $5(0-28)$ & $3(0-22)$ \\
Body mass index (kg/m ${ }^{2}$ ) & $24.8 \pm 1.9$ & $24.7 \pm 2.1$ \\
$\mathrm{HbA}_{1 \mathrm{c}}(\%)$ & $7.6 \pm 0.9$ & $7.3 \pm 0.8$ \\
Smoking, $\mathrm{n}(\%)$ & $28(58)$ & $27(56)$ \\
Retinopathy (\%, none/simplex/proliferative) & $65 / 33 / 2$ & $73 / 21 / 6$ \\
Macroangiopathy (\%) & 21 & 23 \\
Nephropathy (\%, normo-/micro-/macroalbuminuria) & $71 / 25 / 4$ & $73 / 23 / 4$ \\
Neuropathy (\%) & 75 & 71 \\
Prestudy anti-hyperglycaemic treatment (\%) & & 15 \\
Diet only & 19 & 8 \\
Metformin & 13 & 0 \\
Repaglinide & 8 & 63 \\
Sulphonylurea & 44 & 15 \\
Metformin plus sulphonylurea & 17 & 46 \\
$\quad$ Ongoing non-study medication (\%) & & 25 \\
Anti-hypertensive medication & 63 & 46 \\
Lipid-lowering medication & 35 & \\
Aspirin & 44 & \\
\hline
\end{tabular}

Data are expressed as mean \pm SD, median (range) or percentages. Data are stratified according to treatment sequence: repaglinide followed by metformin or vice versa. 
Table 3.2 First- and second-period baseline values of the studied metabolic variables

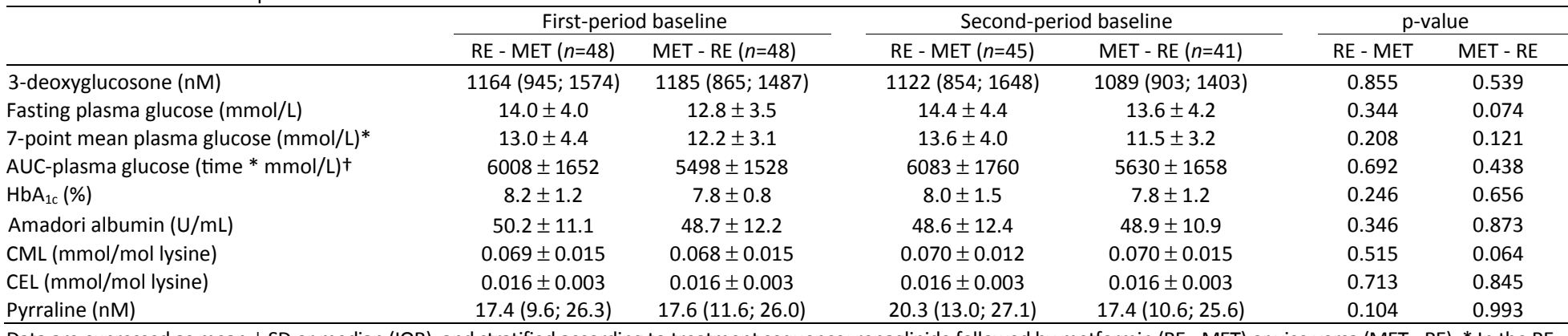

Data are expressed as mean \pm SD or median (IQR), and stratified according to treatment sequence: repaglinide followed by metformin (RE - MET) or vice versa (MET - RE). * In the RE - MET group, $n=36$ and $n=32$ and in the MET - RE group $n=37$ and $n=24$ for the first- and second-period baseline respectively; + In the RE - MET group, $n=46$ and $n=42$ and in the MET RE group $n=45$ and $n=38$ for the first- and second-period baseline respectively. AUC, area under the curve during the $6 \mathrm{~h}$ meal test; $A G E$, advanced glycation endproduct; $C M L, \mathrm{~N}^{\varepsilon}$ (carboxymethyl) lysine; CEL, $\mathrm{N}^{\varepsilon}$ (carboxyethyl) lysine. 
Table 3.2 shows first- (i.e. after one-month run-in on diet-only treatment) and secondperiod (i.e. after one-month washout on diet-only treatment) baseline data of 3DG and the other metabolic variables examined, according to each treatment sequence. No significant differences were found between first- and second-period baseline data.

\section{EFFECTS OF METFORMIN OR REPAGLINIDE TREATMENT ON 3DG}

3DG levels decreased after both metformin [-19.3\% (95\% $\mathrm{Cl}:-23.5 ;-14.8)]$ and repaglinide $[-20.8 \%(-24.9 ;-16.3)]$ treatment (Figure 3.2$)$, but no significant difference was found between the two treatments $[1.8 \%(-3.8 ; 7.8)]$.
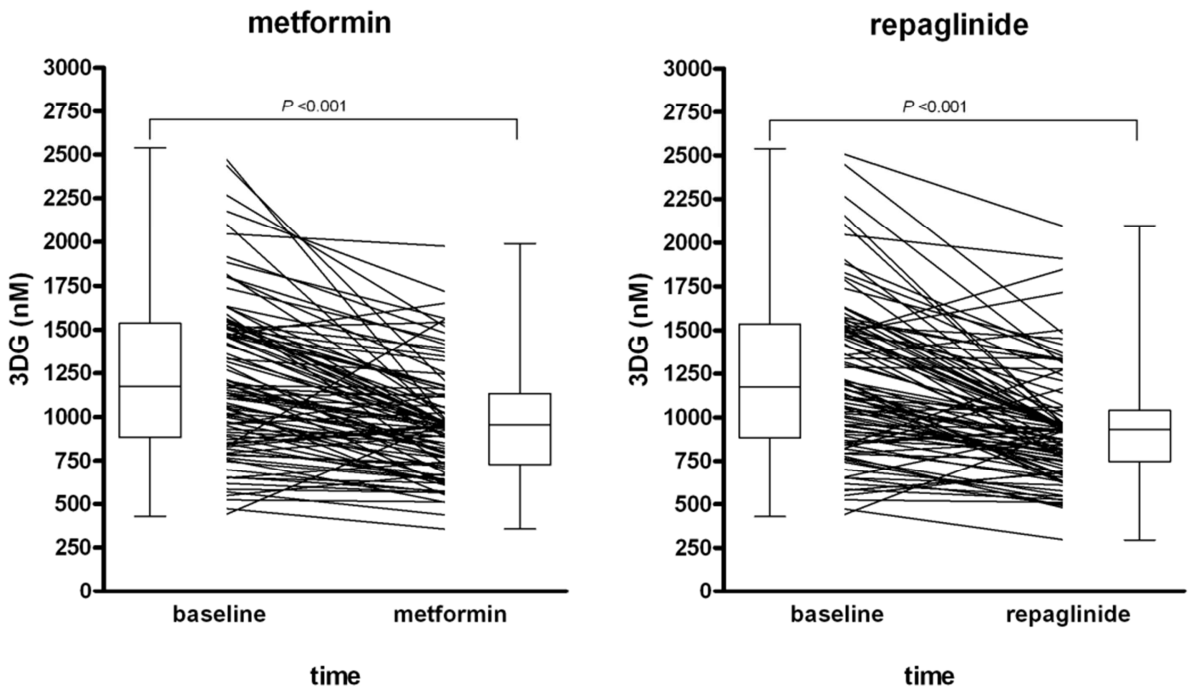

Figure 3.2 Levels of 3-deoxyglucosone (3DG) at baseline and after treatment with metformin or repaglinide.

Boxes indicate the median values and the interquartile ranges and the whiskers represent the minimum and maximum values. P-values for differences between baseline and after 4 months of treatment were calculated using a linear mixed model (see methods section). 


\section{ASSOCIATIONS BETWEEN CHANGES IN GLYCAEMIC VARIABLES AND CHANGES IN 3DG}

To explore a potential pathway through which both treatments could have induced decreases in 3DG levels, we investigated the associations between changes in a range of glycaemic variables and changes in 3DG.

Changes in all glycaemic variables were positively associated with changes in 3DG (Table 3.3). Specifically, for each standard deviation (SD) decrease in FPG, 3DG decreased by $22.7 \%$ (19.0; 26.5). Similarly, 3DG decreased by $20.0 \%(16.2 ; 23.9)$ per SD decrease in 7point MPG, by $22.5 \%(18.6 ; 26.6)$ per SD decrease in AUC-PG and by $17.2 \%(13.8 ; 20.6)$ per $\mathrm{SD}$ decrease in $\mathrm{HbA}_{1 \mathrm{c}}$. Noticeably, the magnitudes of these associations were similar. In addition, for each SD decrease in Amadori albumin, 3DG decreased by $10.9 \%(6.4 ; 15.5)$.

Table 3.3 Associations of changes in glycaemic variables with percent changes in 3DG

\begin{tabular}{lccc}
\hline & \multicolumn{3}{c}{$\Delta$ 3-deoxyglucosone } \\
\cline { 2 - 4 } & $\beta$ & $95 \% \mathrm{Cl}$ & $\mathrm{p}$-value \\
\hline Fasting plasma glucose & 22.7 & $19.0 ; 26.5$ & $<0.001$ \\
$\Delta$ 7-point mean plasma glucose & 20.0 & $16.2 ; 23.9$ & $<0.001$ \\
$\Delta$ AUC-plasma glucose $_{\Delta \mathrm{HbA}_{1 \mathrm{y}}}$ & 22.5 & $18.6 ; 26.6$ & $<0.001$ \\
$\Delta$ Amadori albumin & 17.2 & $13.8 ; 20.6$ & $<0.001$ \\
\hline
\end{tabular}

The regression coefficient $\beta$ represents the percent change (i.e. decrease) in 3DG levels with a change (i.e. decrease) of 1 $\mathrm{SD}$ in the studied glycaemic variable. All analyses were adjusted for treatment type, treatment sequence, the period effect and baseline levels of 3DG and the studied glycaemic variable. $C l$, confidence interval; $A U C$, area under the curve during $6 \mathrm{~h}$ meal test.

\section{ASSOCIATIONS BETWEEN CHANGES IN 3DG AND CHANGES IN BIOMARKERS OF ADVANCED GLYCATION, ENDOTHELIAL DYSFUNCTION AND INFLAMMATION}

To explore potential pathways through which decreases in 3DG levels could influence the cardiovascular risk profile, we investigated the associations between changes in 3DG and changes in biomarkers of advanced glycation, endothelial dysfunction and inflammation.

For each SD decrease in 3DG, CML decreased by 0.17 SD $(0.01 ; 0.33)$, but no associations were found between changes in 3DG and changes in CEL [0.08 SD (-0.07; $0.24)]$ or pyrraline $[-1.8 \%(-11.4 ; 8.8)]$ (Table 3.4). 
Table 3.4 Associations of changes in 3DG with changes in biomarkers of advanced glycation

\begin{tabular}{lccc}
\hline & \multicolumn{3}{c}{$\Delta$ 3-deoxyglucosone } \\
\cline { 2 - 4 } & $\beta$ & $95 \% \mathrm{Cl}$ & $\mathrm{p}$-value \\
\hline$\Delta \mathrm{N}^{\varepsilon}$ (carboxymethyl) lysine (CML) & 0.17 & $0.01 ; 0.33$ & 0.044 \\
$\Delta \mathrm{N}^{\varepsilon}$ (carboxyethyl) lysine (CEL) & 0.08 & $-0.07 ; 0.24$ & 0.286 \\
$\Delta$ Pyrraline & -1.8 & $-11.4 ; 8.8$ & 0.724 \\
\hline
\end{tabular}

The regression coefficient $\beta$ represents the changes (in SD for CML and CEL; in \% for pyrraline) in the studied biomarker of advanced glycation with a change of 1 SD in 3DG levels. All analyses were adjusted for treatment type, treatment sequence, the period effect and baseline levels of 3DG and the studied biomarker of advanced glycation. $\mathrm{Cl}$, confidence interval.

Furthermore, the treatment-induced reduction in 3DG was associated with a reduction in the endothelial markers PAI-1-ag, tPA-ag, sVCAM-1, sE-Selectin, but not with changes in vWf and sICAM-1. In addition, we found that treatment-induced reduction in 3DG was not associated with statistically significant reductions in the inflammatory markers TNF- $\alpha$, CRP, IL-6 and fibrinogen (Table 3.5).

Table 3.5 Associations of changes in 3DG with changes in biomarkers of endothelial dysfunction and low-grade inflammation

\begin{tabular}{lccc}
\hline & \multicolumn{2}{c}{$\Delta$ 3-deoxyglucosone } \\
\cline { 2 - 4 } & $\beta$ & $95 \% \mathrm{Cl}$ & $\mathrm{p}$-value \\
\hline Endothelial dysfunction & & & 0.034 \\
$\Delta$ plasminogen activator inhibitor-1 (PAI-1) & 19.1 & $1.3 ; 40.0$ & 0.029 \\
$\Delta$ tissue-type plasminogen activator antigen (tPA-ag) & 0.17 & $0.02 ; 0.33$ & 0.383 \\
$\Delta$ von Willebrand factor (vWf) & 0.08 & $-0.10 ; 0.25$ & 0.658 \\
$\Delta$ soluble intercellular adhesion molecule-1 (sICAM-1) & 3.9 & $-12.3 ; 23.0$ & 0.034 \\
$\Delta$ soluble vascular cell adhesion molecule-1 (sVCAM-1) & 20.5 & $1.5 ; 43.1$ & 0.044 \\
$\Delta$ soluble E-selectin (sE-selectin) & 19.1 & $0.5 ; 41.2$ & 0.929 \\
Low-grade inflammation & & & 0.065 \\
$\Delta$ C-reactive protein (CRP) & 0.8 & $-16.7 ; 18.2$ & 0.501 \\
$\Delta$ tumour necrosis factor alpha (TNF- $\alpha$ ) & 16.4 & $-1.0 ; 36.8$ & 0.751 \\
$\Delta$ interleukin-6 (IL-6) & -5.3 & $-19.4 ; 11.2$ & $-0.19 ; 0.14$ \\
$\Delta$ fibrinogen & -0.03 & & \\
\hline
\end{tabular}

The regression coefficient $\beta$ represents the changes (in SD for tPA-antigen, vWf, fibrinogen; in \% for PAl-1, sICAM, sVCAM, sE-Selectin, CRP, TNF- $\alpha$, IL-6) in the studied biomarker of endothelial dysfunction or low-grade inflammation with a change of $1 \mathrm{SD}$ in 3DG levels. All analyses were adjusted for treatment type, treatment sequence, the period effect and baseline levels of 3DG and the studied biomarker of endothelial dysfunction or low-grade inflammation. $\mathrm{Cl}$, confidence interval.

\section{ADDITIONAL ANALYSES}

Additional adjustment for changes in body mass, which differed between treatments ${ }^{14}$ and may affect the metabolic variables examined herein, did not substantially influence our results. Specifically, after the additional adjustment for changes in body mass, 3DG 
levels decreased after both metformin $[-18.0 \%(-22.4 ;-13.6)]$ and repaglinide $[-22.0 \%$ ($26.1 ;-17.7)]$ treatments, but no significant difference was found between the two treatments $[-4.8 \%(-10.4 ; 1.1)]$. In addition, adjustment for changes in body mass did not substantially influence the associations between changes in glycaemic variables and biomarkers of advanced glycation, endothelial dysfunction and low-grade inflammation and changes in 3DG (data not shown).

\section{DISCUSSION}

The present single-centre, randomized, double-masked, double-dummy, crossover study of 96 non-obese insulin-naive individuals with type 2 diabetes comparing the effects of metformin and repaglinide treatment on blood levels of 3DG had three main findings. First, 3DG levels decreased to the same extent either after treatment with metformin or with repaglinide. Second, changes in several glycaemic variables, i.e. FPG, 7-point MPG, AUC-PG, $\mathrm{HbA}_{1 \mathrm{c}}$ and Amadori albumin, were strongly and positively associated with these changes in 3DG levels. Third, metformin- and repaglinide-induced changes in 3DG were positively associated with changes in CML (i.e. an AGE that can be formed by 3DG) and several markers of endothelial dysfunction (i.e. PAI-1, tPA-ag, sVCAM-1 and sE-Selectin).

Given the similarities in structure between metformin and aminoguanidine, it has been proposed that metformin reacts with the dicarbonyl groups of alpha-oxoaldehydes in a similar way to aminoguanidine, thereby preventing the toxicity of these alphaoxoaldehydes and the subsequent production of AGEs. ${ }^{9-11}$ In the present study, we indeed found a decrease in 3DG levels after treatment with metformin, which is in agreement with earlier observations of lower levels of alpha-oxoaldehydes and AGEs in patients with type 2 diabetes treated with this drug. ${ }^{9} 11$ Remarkably, we found a similar decrease in 3DG levels after treatment with another anti-hyperglycaemic agent, repaglinide, which is a short-acting insulin secretagogue belonging to the meglitinide analogues.

The different working mechanisms of the anti-hyperglycaemic effects of metformin and repaglinide suggest that improvement in glycaemic control, rather than a specific effect of metformin on quenching of 3DG, results in a decrease in 3DG. This is concordant with a similar $\mathrm{HbA}_{1 \mathrm{c}}$-lowering effect of either treatment as previously reported $^{14}$ and further supported by our current findings showing that changes in 3DG were strongly associated with changes in several glycaemic variables, independently of treatment type, treatment sequence and period effect.

Both metformin ${ }^{12,19}$ and repaglinide 20,21 have been described to hold cardiovascular potentials in patients with type 2 diabetes, though a greater beneficial role has been attributed to metformin, which has also been previously shown in this study. ${ }^{15}$ 
Our current findings showing a similar beneficial effect of both drugs on 3DG levels, which are associated with diabetic microangiopathy, ${ }^{5-7}$ suggest that the metformin- or repaglinide-induced reduction in 3DG may explain part of the beneficial effect of both drugs on the cardiovascular risk profile.

We have also found a strong association between changes in AUC-plasma glucose and changes in $3 D G$. Postprandial rise in $3 D^{6}{ }^{6}$ and the association between postprandial glucose levels and levels of alpha-oxoaldehydes including 3DG have been described previously. ${ }^{22}$ In this regard it is important to emphasize that postprandial fluctuations in glucose levels have been thought to be more strongly associated with complications than high glucose levels in general. ${ }^{23,}{ }^{24}$ Although the exact mechanisms explaining the increased risk for cardiovascular disease due to glucose fluctuations are unknown, increased oxidative stress by glucose fluctuations during postprandial periods seems to be involved. ${ }^{23,24}$ Such increases in oxidative stress may be due to increased formation of alpha-oxoaldehydes. ${ }^{6}$ Therefore, both an increase in oxidative stress and dicarbonyl stress and consequent increased formation of AGEs may affect the vasculature and thus explain the increased cardiovascular risk related to postprandial fluctuations in glucose levels.

Two major pathways for the formation of 3DG have been described. ${ }^{25}$ 3DG can be formed in the polyol pathway, in which 3DG is a hydrolysis product of fructose-3phosphate, and through the Maillard reaction. In the latter, the non-enzymatic glycation of protein amino groups leads to the formation of an Amadori product, which can then undergo multiple dehydration reactions and rearrangements to produce highly reactive carbonyl compounds such as 3DG. In accordance, we found that levels of Amadori albumin decreased after anti-hyperglycaemic treatment and that this change was associated with a decrease in blood levels of 3DG. 3DG can react with free amino groups to form AGEs including CML and pyrraline. ${ }^{25}$ Indeed, our current findings show an association between changes in 3DG and changes in $\mathrm{CML}$ after glucose-lowering treatment. However, we could not find such an association with changes in pyrraline. These differences might be explained by the fact that $\mathrm{CML}$, rather than pyrraline, is a major AGE structure in 3DG-modified proteins. ${ }^{26}$

Endothelial dysfunction and low-grade inflammation have been described as potential pathways to explain the increased risk for cardiovascular disease in both type $1^{27,28}$ and type 2 diabetes ${ }^{29}$ and the increased formation of AGEs could play an important role in these pathways. ${ }^{30,31}$ Indeed, our current results show an association between a glucose lowering treatment-induced reduction in 3DG and a reduction in markers of endothelial dysfunction, but not of low-grade inflammation, which further supports the view that this could constitute a pathway through which inhibition of the glycation pathway may have a beneficial impact on the cardiovascular risk profile. Moreover, from the present study, we previously have shown that metformin had a more pronounced effect to lower such markers of endothelial dysfunction than did repaglinide despite 
overall similar glycaemic control (i.e. independent of glycaemic control). ${ }^{15}$ Thus, together with our present data indicating equal potency of metformin and repaglinide to lower 3DG as well as an association between 3DG and markers of endothelial dysfunction, this suggests that metformin might have a potential beneficial influence on such markers of cardiovascular risk through both shared (glycaemic) and specific (non-glycaemic) pathways.

Some limitations to this study need to be mentioned. First, the lack on a 'nontreatment' or 'diet-only' arm does not enable us a full understanding of the importance of magnitude of the effects observed on 3DG levels after treatment with both metformin and repaglinide. Second, the study was not designed to assess the exact mediating effects of treatment-derived changes in the different glycaemic variables on changes in 3DG. However, the strong and consistent associations between changes in the different variables - regardless of treatment type - and the fact that treatment has a similar effect on both 3DG and other glycaemic variables, suggest that changes in other glycaemic variables, such as plasma glucose levels and $\mathrm{HbA}_{1 \mathrm{c}}$, at least partially explained the reduction in 3DG induced by metformin and repaglinide. Third, the presence of carry-over effects in a trial with a crossover design could constitute such another limitation. However, the two treatment sequence groups (i.e. metformin followed by repaglinide or vice versa) were well balanced and comparable at each baseline measurement, and a possible carry-over effect was adjusted for to the extent it influenced the effect of period and treatment sequence. We analysed between treatment effects as changes from firstperiod baseline. We chose this because the second-period baseline could be biased between treatment sequences by possible differential carry-over effects arising from the two treatments being stopped only one month earlier when completing the firsttreatment period. In contrast, we did not have a specific reason to suspect such potential differential effects from previous glucose-lowering treatments at the first-period baseline (because previous such therapies were equally distributed between treatment sequences (Table 1); $p>0.1$ ). However, additionally using the second-period baseline in the analyses did not substantially influence our results (data not shown). Finally, this trial was carried out with non-obese patients with type 2 diabetes. Therefore, the extent to which our findings may be extrapolated to obese patients remains uncertain. Noteworthy, more than $20 \%$ of Caucasian patients with type 2 diabetes are non-obese. ${ }^{32}$ In these patients and in contrast to those who are obese, solid scientific evidence supporting metformin as 'drug of first choice', as opposed to an insulin secretagogue, is still lacking however.

In conclusion, the improved glycaemic control in type 2 diabetes induced by metformin and repaglinide is associated with a reduction in 3DG levels, which is similar in magnitude for both drugs and associated with reductions in AGEs and endothelial markers. This may constitute a metabolic pathway through which both treatments have a beneficial impact on the cardiovascular risk profile. 


\section{ACKNOWLEDGEMENTS}

We would like to thank the following people for great help and support in carrying out this study:

Bente Blaaholm Nielsen as trial nurse; Merete Frandsen, Ulla Meng Smidt, Birgitte Vilsbøl Hansen, Tina Ragnholm Juhl, Berit Ruud Jensen, Lotte Pietraszek, Ingelise Rossing and Jean Scheijen as laboratory technicians. 


\section{REFERENCES}

1. Brownlee M. Biochemistry and molecular cell biology of diabetic complications. Nature. 2001;414(6865):813-820.

2. Basta G. Receptor for advanced glycation endproducts and atherosclerosis: From basic mechanisms to clinical implications. Atherosclerosis. 2008;196(1):9-21.

3. Yamagishi S, Nakamura K, Matsui T, Ueda S, Noda Y, Imaizumi T. Inhibitors of advanced glycation end products (AGEs): potential utility for the treatment of cardiovascular disease. Cardiovasc Ther. 2008;26(1):50-58.

4. Thornalley PJ, Langborg A, Minhas HS. Formation of glyoxal, methylglyoxal and 3-deoxyglucosone in the glycation of proteins by glucose. Biochem J. 1999;344 Pt 1:109-116.

5. Kusunoki H, Miyata S, Ohara T, Liu BF, Uriuhara A, Kojima $H$, et al. Relation between serum 3deoxyglucosone and development of diabetic microangiopathy. Diabetes Care. 2003;26(6):1889-1894.

6. Schindhelm RK, Alssema M, Scheffer PG, Diamant M, Dekker JM, Barto R, et al. Fasting and postprandial glycoxidative and lipoxidative stress are increased in women with type 2 diabetes. Diabetes Care. 2007;30(7):1789-1794.

7. Beisswenger PJ, Drummond KS, Nelson RG, Howell SK, Szwergold BS, Mauer M. Susceptibility to diabetic nephropathy is related to dicarbonyl and oxidative stress. Diabetes. 2005;54(11):3274-3281.

8. Thornalley PJ. Use of aminoguanidine (Pimagedine) to prevent the formation of advanced glycation endproducts. Arch Biochem Biophys. 2003;419(1):31-40.

9. Beisswenger PJ, Howell SK, Touchette AD, Lal S, Szwergold BS. Metformin reduces systemic methylglyoxal levels in type 2 diabetes. Diabetes. 1999;48(1):198-202.

10. Ruggiero-Lopez D, Lecomte M, Moinet G, Patereau G, Lagarde M, Wiernsperger N. Reaction of metformin with dicarbonyl compounds. Possible implication in the inhibition of advanced glycation end product formation. Biochem Pharmacol. 1999;58(11):1765-1773.

11. Rabbani N, Chittari MV, Bodmer CW, Zehnder D, Ceriello A, Thornalley PJ. Increased glycation and oxidative damage to apolipoprotein B100 of LDL cholesterol in patients with type 2 diabetes and effect of metformin. Diabetes. 2010;59(4):1038-1045.

12. UK Prospective Diabetes Study (UKPDS) Group. Effect of intensive blood-glucose control with metformin on complications in overweight patients with type 2 diabetes (UKPDS 34). UK Prospective Diabetes Study (UKPDS) Group. Lancet. 1998;352(9131):854-865.

13. Lund SS, Tarnow L, Frandsen M, Smidt UM, Pedersen O, Parving HH, et al. Impact of metformin versus the prandial insulin secretagogue, repaglinide, on fasting and postprandial glucose and lipid responses in nonobese patients with type 2 diabetes. Eur J Endocrinol. 2008;158(1):35-46.

14. Lund SS, Tarnow L, Stehouwer CD, Schalkwijk CG, Frandsen M, Smidt UM, et al. Targeting hyperglycaemia with either metformin or repaglinide in non-obese patients with type 2 diabetes: results from a randomized crossover trial. Diabetes Obes Metab. 2007;9(3):394-407.

15. Lund SS, Tarnow L, Stehouwer CD, Schalkwijk CG, Teerlink T, Gram J, et al. Impact of metformin versus repaglinide on non-glycaemic cardiovascular risk markers related to inflammation and endothelial dysfunction in non-obese patients with type 2 diabetes. Eur J Endocrinol. 2008;158(5):631-641.

16. Schalkwijk CG, Posthuma N, ten Brink HJ, ter Wee PM, Teerlink T. Induction of 1,2-dicarbonyl compounds, intermediates in the formation of advanced glycation end-products, during heat-sterilization of glucosebased peritoneal dialysis fluids. Perit Dial Int. 1999;19(4):325-333.

17. Schalkwijk CG, Ligtvoet N, Twaalfhoven H, Jager A, Blaauwgeers HG, Schlingemann RO, et al. Amadori albumin in type 1 diabetic patients: correlation with markers of endothelial function, association with diabetic nephropathy, and localization in retinal capillaries. Diabetes. 1999;48(12):2446-2453.

18. Teerlink T, Barto R, Ten Brink HJ, Schalkwijk CG. Measurement of Nepsilon-(carboxymethyl)lysine and Nepsilon-(carboxyethyl)lysine in human plasma protein by stable-isotope-dilution tandem mass spectrometry. Clin Chem. 2004;50(7):1222-1228.

19. De Jager J, Kooy A, Lehert P, Bets D, Wulffele MG, Teerlink T, et al. Effects of short-term treatment with metformin on markers of endothelial function and inflammatory activity in type 2 diabetes mellitus: a randomized, placebo-controlled trial. J Intern Med. 2005;257(1):100-109. 
20. Esposito K, Giugliano D, Nappo F, Marfella R. Regression of carotid atherosclerosis by control of postprandial hyperglycemia in type 2 diabetes mellitus. Circulation. 2004;110(2):214-219.

21. Manzella D, Grella R, Abbatecola AM, Paolisso G. Repaglinide administration improves brachial reactivity in type 2 diabetic patients. Diabetes Care. 2005;28(2):366-371.

22. Beisswenger PJ, Howell SK, O'Dell RM, Wood ME, Touchette AD, Szwergold BS. alpha-Dicarbonyls increase in the postprandial period and reflect the degree of hyperglycemia. Diabetes Care. 2001;24(4):726-732.

23. Ceriello A, Hanefeld M, Leiter L, Monnier L, Moses A, Owens D, et al. Postprandial glucose regulation and diabetic complications. Arch Intern Med. 2004;164(19):2090-2095.

24. Monnier L, Mas E, Ginet C, Michel F, Villon L, Cristol JP, et al. Activation of oxidative stress by acute glucose fluctuations compared with sustained chronic hyperglycemia in patients with type 2 diabetes. Jama. 2006;295(14):1681-1687.

25. Niwa T. 3-Deoxyglucosone: metabolism, analysis, biological activity, and clinical implication. J Chromatogr B Biomed Sci Appl. 1999;731(1):23-36.

26. Jono T, Nagai R, Lin X, Ahmed N, Thornalley PJ, Takeya M, et al. Nepsilon-(Carboxymethyl)lysine and 3-DGimidazolone are major AGE structures in protein modification by 3-deoxyglucosone. J Biochem. 2004;136(3):351-358.

27. Schram MT, Chaturvedi N, Schalkwijk C, Giorgino F, Ebeling P, Fuller JH, et al. Vascular risk factors and markers of endothelial function as determinants of inflammatory markers in type 1 diabetes: the EURODIAB Prospective Complications Study. Diabetes Care. 2003;26(7):2165-2173.

28. Schram MT, Chaturvedi N, Schalkwijk CG, Fuller JH, Stehouwer CD. Markers of inflammation are crosssectionally associated with microvascular complications and cardiovascular disease in type 1 diabetes--the EURODIAB Prospective Complications Study. Diabetologia. 2005;48(2):370-378.

29. de Jager J, Dekker JM, Kooy A, Kostense PJ, Nijpels G, Heine RJ, et al. Endothelial dysfunction and low-grade inflammation explain much of the excess cardiovascular mortality in individuals with type 2 diabetes: the Hoorn Study. Arterioscler Thromb Vasc Biol. 2006;26(5):1086-1093.

30. Brouwers O, Niessen PM, Haenen G, Miyata T, Brownlee M, Stehouwer CD, et al. Hyperglycaemia-induced impairment of endothelium-dependent vasorelaxation in rat mesenteric arteries is mediated by intracellular methylglyoxal levels in a pathway dependent on oxidative stress. Diabetologia. 2010;53(5):9891000.

31. Stirban A, Negrean M, Stratmann B, Gawlowski T, Horstmann T, Gotting C, et al. Benfotiamine prevents macro- and microvascular endothelial dysfunction and oxidative stress following a meal rich in advanced glycation end products in individuals with type 2 diabetes. Diabetes Care. 2006;29(9):2064-2071.

32. Dalton M, Cameron AJ, Zimmet PZ, Shaw JE, Jolley D, Dunstan DW, et al. Waist circumference, waist-hip ratio and body mass index and their correlation with cardiovascular disease risk factors in Australian adults. J Intern Med. 2003;254(6):555-563. 



\section{CHAPTER 4}

IRBESARTAN TREATMENT DOES NOT INFLUENCE PLASMA LEYELS OF THE ADVANCED S̆LYCATION ENDPRODUCTS $K^{\varepsilon}$ (1-CARBOXYMETHYL]LYSIME AND $\mathrm{N}^{\varepsilon}$ (1-CARBOXYETHYL]LYSINE IN PATIEWTS WITH TYPE 2 DIABETES AND MICROALBUMINURIA.

A RANDOMIZED CONTROLLED TRIAL

LIAN EMSELEN. FREDERIK PERSSOH, ISABEL FERREIRA, PETER ROSSING

PETER HOVIKD. TOM TEERLINK, COEN DA STEHOULYER, HANS-HENRIK PARVINS. CASPER is SCHALKX:IJIK

NEPHROL DIAL TRAASPLANT. 2011 NOV:26(11):3573-7 


\section{ABSTRACT}

\section{Background}

In vitro and animal experiments have shown inhibiting effects of angiotensin receptor blockers (ARBs) on the formation of advanced glycation endproducts (AGEs), which are known to be involved in the development of cardiovascular complications in diabetes. However, sufficient human data to confirm such beneficial effects of ARBs on AGEs are lacking. Therefore, we investigated the effects of irbesartan treatment on plasma levels of the AGEs $N^{\varepsilon}\left(1\right.$-carboxymethyl)lysine (CML) and $N^{\varepsilon}(1$-carboxyethyl)lysine (CEL) in hypertensive patients with type 2 diabetes and microalbuminuria.

\section{Methods}

We analysed data from a multicentre, double-blind, parallel, randomized controlled trial in patients with type 2 diabetes and microalbuminuria, the primary goal of which was to examine the renoprotective effects of irbesartan treatment (150 or $300 \mathrm{mg}$ daily). Secondary endpoints included plasma CML and CEL in the treatment arm receiving $300 \mathrm{mg}$ irbesartan ( $n=139)$ and in the placebo group $(n=125)$. Effects of treatment at 1- and 2-year follow-up were analyzed by means of generalized estimating equations according to an intention-to-treat principle.

\section{Results}

Levels of CML and CEL did not differ between groups at baseline. No significant changes were observed in CML and CEL over time in either group and there was no effect of treatment on CML and CEL at any time-point. Mean differences for the irbesartan vs. placebo group over time were $-0.96 \mu \mathrm{mol} / \mathrm{mol}$ lysine $(95 \% \mathrm{Cl}:-3.43 ; 1.51)$ for $\mathrm{CML}$ and $0.10 \mu \mathrm{mol} / \mathrm{mol}$ lysine $(-0.76 ; 0.56)$ for CEL.

\section{Conclusions}

Long-term irbesartan treatment does not influence plasma levels of the AGEs CML and CEL in patients with type 2 diabetes and microalbuminuria. 


\section{INTRODUCTION}

Increased formation of advanced glycation endproducts (AGEs) constitutes a potential mechanism by which hyperglycaemia and its immediate biochemical sequelae induce micro- and macrovascular complications in diabetes. ${ }^{1}$ Although the exact mechanisms through which AGEs are involved in vascular complications are still under debate, direct modification of extracellular matrix proteins, resulting in collagen cross-links and increased vascular stiffness is likely involved. ${ }^{1}$ In addition, binding of AGEs to a variety of cell-surface receptors, such as the receptor for advanced glycation endproducts (RAGE) could trigger signal transduction pathways and the production of reactive oxygen species, resulting in proinflammatory cellular responses. ${ }^{2}$ Several potential AGE inhibitors have thus been developed, although the clinical value remains to be established. ${ }^{3}$

The renin-angiotensin-aldosterone system (RAAS), which is upregulated in diabetes, also plays an important role in the development of renal and cardiovascular disease. ${ }^{4-6}$ Blockade of the RAAS with the use of angiotensin receptor blockers (ARBs) reduces the progression of nephropathy and other cardiovascular outcomes in patients with type 2 diabetes. 7,8 Interestingly, it has been shown that several ARBs attenuate the in vitro production of AGEs and dicarbonyl compounds, which are reactive intermediates in the formation of AGEs. ${ }^{9}$ In addition, several animal experiments have also shown a reduction in AGE accumulation in the kidneys ${ }^{10-14}$ and eyes ${ }^{15}$ after treatment with ARBs. These observations led to the hypothesis that AGE-inhibiting effects may contribute, at least in part, to the beneficial effects of ARBs on diabetic micro- and macrovascular complications. ${ }^{12,15}$ However, sufficient clinical evidence from human studies to sustain this contention is still lacking. Indeed, decreases in circulating ${ }^{16-18}$ or urinary ${ }^{18}$ AGEs in patients with type 2 diabetes after treatment with ARBs have been observed in very small clinical studies lacking control groups, but no such effects on circulating AGEs were confirmed in a larger sub-sample of a placebo-controlled trial ${ }^{19}$ neither by our previous observations on AGE peptides, ${ }^{20}$ which however, do not represent AGEs appropriately as it has been shown that AGE peptides measurements only reflect total low molecular weight fluorophores, which are not only due to AGEs or even AGE peptides and could show nonAGE related effects. ${ }^{21}$

In view of these considerations, we have now investigated the long-term effects of irbesartan treatment as compared with placebo on plasma levels of the AGEs $N^{\varepsilon}(1-$ carboxymethyl)lysine (CML) and $\mathrm{N}^{\varepsilon}$ (1-carboxyethyl)lysine (CEL), as determined by the gold standard technique for measuring plasma AGEs (i.e. HPLC-tandem MS) in a randomized controlled trial in patients with type 2 diabetes and microalbuminuria, the primary goal of which was to examine the renoprotective effects of irbesartan treatment. ${ }^{8}$ 


\section{METHODS}

\section{STUDY DESIGN AND PARTICIPANTS}

The IRMA 2 study was a 2-year multicentre, double-blind, parallel randomized controlled trial in patients with type 2 diabetes and microalbuminuria which main goal was to compare the effects of irbesartan (150 or $300 \mathrm{mg}$ once daily) versus placebo, in addition to conventional antihypertensive treatment, on the development of overt nephropathy. 8 Briefly, we enrolled patients with type 2 diabetes (diagnosed according to the WHO criteria $^{22}$ ) and hypertension (defined as mean systolic blood pressure $>135 \mathrm{~mm} \mathrm{Hg}$ and/or mean diastolic blood pressure $>85 \mathrm{~mm} \mathrm{Hg}$ in two out of three consecutive measurements one week apart) and persistent microalbuminuria (defined as an albumin excretion rate of 20-200 $\mathrm{\mu g} / \mathrm{min}$ in two out of three consecutive overnight urine samples), and a serum creatinine concentration of $\leq 1.5 \mathrm{mg} / \mathrm{dL}$ for men and $\leq 1.1 \mathrm{mg} / \mathrm{dL}$ for women. A total of 590 (186 women) patients, aged 30-70 years, were included and randomly assigned to receive $150 \mathrm{mg}$ irbesartan once daily ( $n=195), 300 \mathrm{mg}$ irbesartan once daily $(n=194)$, or matching placebo $(n=201)$. The study protocol was in accordance with the Declaration of Helsinki and approved by the institutional review board at each centre. All patients gave written informed consent.

Results regarding the primary endpoint, ${ }^{8}$ and other secondary endpoints such as markers of inflammation and endothelial dysfunction, ${ }^{20}$ have been reported previously. The aim of the present study was to evaluate the effect of irbesartan treatment on plasma levels of the AGEs CML and CEL. Samples for their assessment at baseline and at 1- and 2years follow-up were available from 139 patients in the treatment arm receiving $300 \mathrm{mg}$ irbesartan and from 125 patients receiving placebo, because, unfortunately, all samples from the $150 \mathrm{mg}$ irbesartan group were discarded by error. However, in the initial analyses of this study, no significant effects of $150 \mathrm{mg}$ irbesartan treatment versus placebo were found on the development of nephropathy, creatinine clearance or blood pressure. $^{8}$

\section{BIOCHEMICAL MEASUREMENTS}

$\mathrm{HbA}_{1 \mathrm{c}}$ was measured by ion-exchange HPLC. ${ }^{23}$ Serum creatinine concentration was measured by the Jaffe reaction with the use of a Hoffmann-LaRoche $\mathrm{kit}^{24}$ and creatinine clearance (in $\mathrm{mL} / \mathrm{min} / 1.73 \mathrm{~m}^{2}$ body surface area) was estimated with the Cockcroft-Gault equation $\left(\left[(140 \text {-age })^{*}\right.\right.$ body mass*(0.85 if female $\left.)\right] /(72 *$ serum creatinine in $\left.\mathrm{mg} / \mathrm{dL})\right)$. These 
variables were evaluated at the time of randomization, 2 and 4 weeks after randomization, and at 3,6,12,18, and 22-24 months. ${ }^{20}$

$\mathrm{CML}$ and CEL residues in plasma proteins were measured using $\mathrm{HPLC}$-tandem $\mathrm{MS}^{25}$ with inter-assay coefficients of variation being 5.2\% for CML and 3.6\% for CEL. Laboratory technicians were blinded to the allocated treatment and clinical status of the patients.

\section{STATISTICS}

Variables with skewed distribution (i.e. creatinine clearance) were log transformed prior to further analyses.

The effects of treatment with irbesartan on plasma levels of AGEs over time were examined according to the intention-to-treat principle and with the use of generalized estimating equations (GEE). This is a regression model for analyses of repeated measurements that adjusts for the correlation between repeated observations within individuals. We analyzed the mean differences in plasma CML and CEL between the irbesartan treatment and the placebo groups using a first model only including group (i.e. irbesartan or placebo) and time (treated as a categorical variable) as determinants of plasma CML or CEL. We then added interaction terms between group and time to the initial model, in order to assess the exact differences in CML and CEL between groups at each time-point. In all GEE analyses an exchangeable correlation structure was used which was deemed the most appropriate after examination of the inter-period correlation matrices of the dependent variables.

All analyses were performed with the use of STATA 9.0 (Stata Corp., College Station, TX, USA).

\section{RESULTS}

Baseline characteristics are shown in Table 4.1. Plasma levels of CML and CEL, as well as all other patients' characteristics, did not differ between groups. A more detailed description of patients' characteristics, such as ongoing use of non-study medication and adverse events during the trial has been presented elsewhere. ${ }^{8}$ 
Table 4.1 Clinical characteristics of the study participants at baseline

\begin{tabular}{|c|c|c|}
\hline & $\begin{array}{c}300 \text { mg irbesartan } \\
(n=139)\end{array}$ & $\begin{array}{l}\text { Placebo } \\
(n=125)\end{array}$ \\
\hline Men/women, $\mathrm{n}$ & $98 / 41$ & $83 / 42$ \\
\hline Age (years) & $57.3 \pm 7.9$ & $58.2 \pm 9.1$ \\
\hline Known duration of diabetes (years) & $7(4-12)$ & $8(4-15)$ \\
\hline $\mathrm{HbA}_{1 \mathrm{c}}(\%)$ & $6.9 \pm 1.7$ & $7.1 \pm 1.7$ \\
\hline Body mass index $\left(\mathrm{kg} / \mathrm{m}^{2}\right)$ & $29.8 \pm 4.3$ & $30.5 \pm 4.3$ \\
\hline Total cholesterol (mmol/L) & $5.7 \pm 1.1$ & $5.8 \pm 1.1$ \\
\hline Current smoking ( $\mathrm{n}(\%))$ & $22(15.8)$ & $25(20.0)$ \\
\hline Systolic blood pressure ( $\mathrm{mm} \mathrm{Hg}$ ) & $154 \pm 13$ & $154 \pm 15$ \\
\hline Diastolic blood pressure (mm Hg) & $92 \pm 10$ & $90 \pm 8$ \\
\hline Pulse pressure $(\mathrm{mm} \mathrm{Hg})$ & $62 \pm 12$ & $64 \pm 14$ \\
\hline Creatinine clearance $\left(\mathrm{ml} / \mathrm{min} / 1.73 \mathrm{~m}^{2}\right.$ body surface area) & $106(91-123)$ & $107(86-127)$ \\
\hline Urinary albumin excretion rate (mg/24h) & $52(35-83)$ & $50(31-80)$ \\
\hline $\mathrm{N}^{\varepsilon}$ (1-carboxymethyl) lysine (CML in $\mu \mathrm{mol} / \mathrm{mol}$ lysine) & $49.0 \pm 14.9$ & $49.9 \pm 11.9$ \\
\hline $\mathrm{N}^{\varepsilon}$ (1-carboxyethyl) lysine (CEL in $\mu \mathrm{mol} / \mathrm{mol}$ lysine) & $13.2 \pm 4.8$ & $13.2 \pm 3.2$ \\
\hline
\end{tabular}

Data are expressed as mean $\pm \mathrm{SD}$, median (interquartile range) or percentages. Data are stratified according to treatment group: $300 \mathrm{mg}$ irbesartan or placebo.

\section{EFFECTS OF IRBESARTAN TREATMENT ON PLASMA LEVELS OF CML AND CEL}

Plasma levels of CML and CEL did not change significantly over time in either group and did not differ between groups (Fig. 4.1). Specifically, overall differences between irbesartan versus placebo throughout the course of follow-up were $-0.96 \mu \mathrm{mol} / \mathrm{mol}$ lysine (95\% $\mathrm{Cl}:-3.43 ; 1.51)$ for $\mathrm{CML}$ and $-0.10 \mu \mathrm{mol} / \mathrm{mol}$ lysine $(-0.76 ; 0.56)$ for $\mathrm{CEL}$, and these effects did also not differ at any time-point ( $P$-values for interaction between group and time were all $\geq 0.674$, Table 4.2 ).

CML

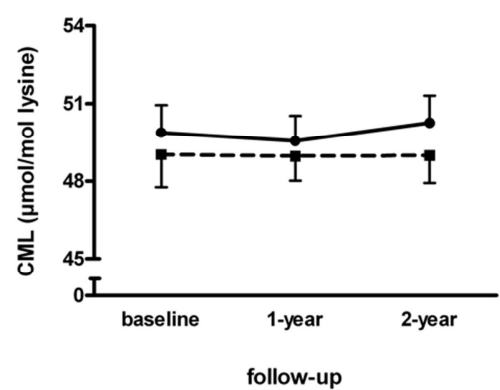

CEL

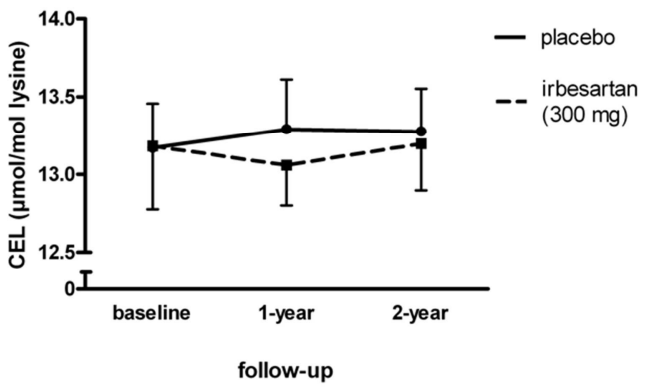

Figure 4.1 Means and standard errors of plasma levels of CML and CEL at baseline, 1-year and 2-year follow-up for the irbesartan treatment group and the placebo group 


\section{ADDITIONAL ANALYSES}

Additional adjustments for age, sex and duration of diabetes (time-independent covariates) and $\mathrm{HbA}_{1 c}$, creatinine clearance and $\mathrm{BMI}$ (time-dependent covariates) did not materially change our results (data not shown).

Table 4.2 Regression coefficients for the effects of irbesartan treatment on plasma levels of CML and CEL as estimated by GEE analyses

\begin{tabular}{|c|c|c|c|c|}
\hline & & $\beta$ & $95 \% \mathrm{Cl}$ & P-value \\
\hline \multirow[t]{8}{*}{ CML } & Intercept: 49.899 & & & \\
\hline & Group: irbesartan vs. placebo & -0.895 & $-4.117 ; 2.328$ & 0.586 \\
\hline & Time & & & \\
\hline & 1 year & -0.364 & $-2.061 ; 1.333$ & 0.674 \\
\hline & 2 year & 0.393 & $-1.356 ; 2.141$ & 0.660 \\
\hline & Group*Time interaction & & & \\
\hline & 1 year & 0.298 & $-2.429 ; 3.026$ & 0.830 \\
\hline & 2 year & -0.520 & $-3.415 ; 2.375$ & 0.725 \\
\hline \multirow[t]{8}{*}{ CEL } & Intercept: 13.188 & & & \\
\hline & Group: irbesartan vs. placebo & -0.010 & $-0.980 ; 0.960$ & 0.983 \\
\hline & Time & & & \\
\hline & 1 year & 0.104 & $-0.450 ; 0.707$ & 0.736 \\
\hline & 2 year & 0.083 & $-0.436 ; 0.602$ & 0.753 \\
\hline & Group*Time interaction & & & \\
\hline & 1 year & -0.214 & $-1.211 ; 0.783$ & 0.674 \\
\hline & 2 year & -0.058 & $-1.070 ; 0.954$ & 0.910 \\
\hline
\end{tabular}

The Intercept represents the plasma AGE levels (in $\mu \mathrm{mol} / \mathrm{mol}$ lysine) for the placebo group at baseline; the regression coefficient $(\beta)$ for the Group variable represents the difference in AGE levels between irbesartan treatment vs. placebo groups at baseline; the $\beta$ for the Time variable (i.e. 1-year and 2-year follow-up) represents the change over time for the placebo group (vs. baseline); the $\beta$ for the Group*Time interactions represents the difference in AGE levels between the irbesartan treatment vs. placebo groups at 1-year and 2-year follow-up vs. baseline, respectively.

On the basis of these equations, the mean levels of CML at $1-y r$ follow-up were 49.535 (=49.899 - 0.364) $\mu \mathrm{mol} / \mathrm{mol}$ lysine in the placebo group and $48.938(=49.899-0.895-0.364+0.298) \mu \mathrm{mol} / \mathrm{mol}$ lysine in the irbesartan group.

\section{DISCUSSION}

The present multicentre, double-blind, randomized controlled trial in patients with type 2 diabetes and microalbuminuria comparing the effects of treatment with irbesartan (300 mg once daily) versus placebo in addition to conventional antihypertensive treatment ${ }^{8,20}$ showed no treatment effects of irbesartan on plasma levels of the AGES CML and CEL.

Blockade of the RAAS system has been shown to reduce progression of nephropathy and cardiovascular outcomes in patients with type 2 diabetes. ${ }^{7,8}$ The observation in several in vitro ${ }^{9}$ and animal experimental ${ }^{10-15}$ studies showing inhibiting effects of ARBs on AGEs and their sequelae, led to the hypothesis that such AGE inhibiting effects could explain, at least in part, the beneficial effects of ARBs on renal and cardiovascular 
outcomes in patients with type 2 diabetes. ${ }^{12,15}$ Specifically, such beneficial effects could be due to the radical scavenging ${ }^{13,26}$ and metal chelating properties ${ }^{26}$ of ARBs, to downregulation of RAGE expression ${ }^{12}$ and/or to restoration of glyoxalase 1 function. ${ }^{27}$ However, although several small clinical studies investigating the AGE inhibiting effects of ARBs have also shown decreases in circulating ${ }^{16-18}$ or urinary ${ }^{18}$ AGEs in patients with type 2 diabetes, Busch et al. ${ }^{19}$ showed no differences in plasma CML and pentosidine after 2 years of treatment with irbesartan vs. placebo in patients with type 2 diabetes. Although our previously published results on AGE peptides ${ }^{20}$ were in line with these results, it is currently known that AGE peptides measurements only reflect total low molecular weight fluorophores, which are not only due to AGEs or even AGE peptides and could show nonAGE related effects. ${ }^{21}$ Our current results in a larger, long-lasting randomized controlled trial using the gold standard for the measurement of plasma AGEs (i.e. HPLC-tandem MS, as opposed to former studies that used ELISA techniques ${ }^{16-19}$ or AGE peptides ${ }^{20}$ ) are in line with the study of Busch et al. Altogether, these findings suggest that AGE-inhibiting effects of ARBs may be restricted to experimental models of diabetes. Therefore, the beneficial effects of ARBs on the renal and cardiovascular system in patients with type 2 diabetes could more likely be explained by mechanisms other than inhibition of advanced glycation. However, we cannot fully discard the possibility that the discrepancy between our current results and the beneficial effects previously shown in in vitro and animal experiments lies in the type of tissue examined. It remains possible that the potential beneficial effects of ARBs, as seen in the kidneys ${ }^{10-13}$ and eyes ${ }^{15}$ in experimental models of diabetes, may not translate to changes in plasma levels of CML and CEL.

A possible limitation to the present study lies in the fact that it was not specifically designed to assess the effects of irbesartan on plasma AGEs. However, given its design, size and precise measurement of plasma AGEs, it provides the best evidence so far with this regard. To further clarify the exact clinical impact, if any, of ARBs on advanced glycation, future research including more and different measurements of advanced glycation (e.g. free methylglyoxal, intracellular AGE concentrations or tissue AGE accumulation), which may better reflect the advanced glycation processes that may be affected by treatment with ARBs, is needed.

In conclusion, long-term irbesartan treatment does not influence plasma levels of the AGEs CML and CEL in hypertensive patients with type 2 diabetes and microalbuminuria. 


\section{ACKNOWLEDGEMENTS}

The authors thank Rob Barto from the Department of Clinical Chemistry, VU University Medical Center in Amsterdam, the Netherlands, for expert technical assistance. 


\section{REFERENCES}

1. Brownlee M. Biochemistry and molecular cell biology of diabetic complications. Nature. 2001;414(6865):813-820.

2. Basta G. Receptor for advanced glycation endproducts and atherosclerosis: From basic mechanisms to clinical implications. Atherosclerosis. 2008;196(1):9-21.

3. Yamagishi S, Nakamura K, Matsui T, Ueda S, Noda Y, Imaizumi T. Inhibitors of advanced glycation end products (AGEs): potential utility for the treatment of cardiovascular disease. Cardiovasc Ther. 2008;26(1):50-58.

4. Volpe M, Savoia C, De Paolis P, Ostrowska B, Tarasi D, Rubattu S. The renin-angiotensin system as a risk factor and therapeutic target for cardiovascular and renal disease. J Am Soc Nephrol. 2002;13 Suppl 3:S173178.

5. Ruiz-Ortega M, Lorenzo O, Ruperez M, Esteban V, Suzuki Y, Mezzano S, et al. Role of the renin-angiotensin system in vascular diseases: expanding the field. Hypertension. 2001;38(6):1382-1387.

6. Hoogwerf BJ. Renin-angiotensin system blockade and cardiovascular and renal protection. Am J Cardiol. 2010;105(1 Suppl):30A-35A.

7. Brenner BM, Cooper ME, de Zeeuw D, Keane WF, Mitch WE, Parving HH, et al. Effects of losartan on renal and cardiovascular outcomes in patients with type 2 diabetes and nephropathy. $\mathrm{N}$ Engl J Med. 2001;345(12):861-869.

8. Parving HH, Lehnert H, Brochner-Mortensen J, Gomis R, Andersen S, Arner P. The effect of irbesartan on the development of diabetic nephropathy in patients with type 2 diabetes. N Engl J Med. 2001;345(12):870878.

9. Miyata T, van Ypersele de Strihou C, Ueda Y, Ichimori K, Inagi R, Onogi H, et al. Angiotensin II receptor antagonists and angiotensin-converting enzyme inhibitors lower in vitro the formation of advanced glycation end products: biochemical mechanisms. J Am Soc Nephrol. 2002;13(10):2478-2487.

10. Forbes JM, Thomas MC, Thorpe SR, Alderson NL, Cooper ME. The effects of valsartan on the accumulation of circulating and renal advanced glycation end products in experimental diabetes. Kidney Int Suppl. 2004(92):S105-107.

11. Nangaku M, Miyata $T$, Sada $T$, Mizuno $M$, Inagi $R$, Ueda $Y$, et al. Anti-hypertensive agents inhibit in vivo the formation of advanced glycation end products and improve renal damage in a type 2 diabetic nephropathy rat model. J Am Soc Nephrol. 2003;14(5):1212-1222.

12. Fan Q, Liao J, Kobayashi M, Yamashita M, Gu L, Gohda T, et al. Candesartan reduced advanced glycation end-products accumulation and diminished nitro-oxidative stress in type 2 diabetic KK/Ta mice. Nephrol Dial Transplant. 2004;19(12):3012-3020.

13. Izuhara Y, Sada T, Yanagisawa H, Koike H, Ohtomo S, Dan T, et al. A novel Sartan derivative with very low angiotensin II type 1 receptor affinity protects the kidney in type 2 diabetic rats. Arterioscler Thromb Vasc Biol. 2008;28(10):1767-1773.

14. Thomas MC, Tikellis C, Burns WM, Bialkowski K, Cao Z, Coughlan MT, et al. Interactions between renin angiotensin system and advanced glycation in the kidney. J Am Soc Nephrol. 2005;16(10):2976-2984.

15. Sugiyama T, Okuno T, Fukuhara M, Oku H, Ikeda T, Obayashi H, et al. Angiotensin II receptor blocker inhibits abnormal accumulation of advanced glycation end products and retinal damage in a rat model of type 2 diabetes. Exp Eye Res. 2007;85(3):406-412.

16. Komiya N, Hirose H, Saisho Y, Saito I, Itoh H. Effects of 12-month valsartan therapy on glycation and oxidative stress markers in type 2 diabetic subjects with hypertension. Int Heart J. 2008;49(6):681-689.

17. Saisho $\mathrm{Y}$, Komiya N, Hirose H. Effect of valsartan, an angiotensin II receptor blocker, on markers of oxidation and glycation in Japanese type 2 diabetic subjects: blood pressure-independent effect of valsartan. Diabetes Res Clin Pract. 2006;74(2):201-203.

18. Monacelli F, Poggi A, Storace D, Durante A, Traverso N, Viviani GL, et al. Effects of valsartan therapy on protein glycoxidation. Metabolism. 2006;55(12):1619-1624.

19. Busch M, Franke S, Wolf G, Rohde RD, Stein G. Serum levels of the advanced glycation end products Nepsilon-carboxymethyllysine and pentosidine are not influenced by treatment with the angiotensin 
receptor II type 1 blocker irbesartan in patients with type 2 diabetic nephropathy and hypertension. Nephron Clin Pract. 2008;108(4):c291-297.

20. Persson F, Rossing $P$, Hovind $P$, Stehouwer CD, Schalkwijk C, Tarnow L, et al. Irbesartan treatment reduces biomarkers of inflammatory activity in patients with type 2 diabetes and microalbuminuria: an IRMA 2 substudy. Diabetes. 2006;55(12):3550-3555.

21. Ahmed N, Thornalley PJ. Advanced glycation endproducts: what is their relevance to diabetic complications? Diabetes Obes Metab. 2007;9(3):233-245.

22. WHO study group. Diabetes Mellitus. WHO Tech Rep Ser. 1985;727:1-104.

23. Goldstein DE, Little RR, Wiedmeyer HM, England JD, McKenzie EM. Glycated hemoglobin: methodologies and clinical applications. Clin Chem. 1986;32(10 Suppl):B64-70.

24. Hofmann W, Guder WG. Preanalytical and analytical factors in determination of urinary lgG, albumin, a1microglobulin and retinol-binding protein with the Behring nephelometer system (BNS). Lab Med. 1989;13:470-478.

25. Teerlink T, Barto R, Ten Brink HJ, Schalkwijk CG. Measurement of Nepsilon-(carboxymethyl)lysine and Nepsilon-(carboxyethyl)lysine in human plasma protein by stable-isotope-dilution tandem mass spectrometry. Clin Chem. 2004;50(7):1222-1228.

26. Miyata T, van Ypersele de Strihou C. Angiotensin II receptor blockers and angiotensin converting enzyme inhibitors: implication of radical scavenging and transition metal chelation in inhibition of advanced glycation end product formation. Arch Biochem Biophys. 2003;419(1):50-54.

27. Miller AG, Tan G, Binger KJ, Pickering RJ, Thomas MC, Nagaraj RH, et al. Candesartan attenuates diabetic retinal vascular pathology by restoring glyoxalase-I function. Diabetes. 2010;59(12):3208-3215. 

PART II

THE GLYCATION PATHWAY AND CARDIOVASCULAR DISEASE 


\section{THE GLYCATION PATHWAY AND CARDIOVASCULAR DISEASE}

The importance of the glycation pathway in the pathogenesis of diabetic vascular complications has been well established in numerous experimental studies, which have linked AGE accumulation in tissues to development of vascular complications and which have shown reductions in vascular complications with (specific) AGE-inhibiting interventions. However, epidemiological evidence on the role of the glycation pathway in type 2 diabetes and its micro- and macrovascular complications in humans is largely lacking.

In the second part of this thesis, we therefore aimed to investigate, in two Dutch observational cohort studies (i.e. the Hoorn and CODAM studies), the role of the glycation pathway in (diabetes-related) cardiovascular disease. To this aim we investigated associations of measures of the glycation pathway with cardiovascular disease and surrogate markers thereof.

The main reason for the use of surrogate markers over (cardiovascular) morbidity and mortality as outcome variables in trials and/or (prospective) observational studies is the considerable reduction in sample size and possibly in duration of follow-up necessary. In addition, the development of cardiovascular events is a heterogeneous process and measurement of surrogate markers of distinct potentially underlying pathophysiological mechanisms (e.g. atherosclerosis and/or arterial stiffness) enables the study of pathways (e.g. glycation) involved in these specific and distinct mechanisms.

Atherosclerosis is the major underlying cause of cardiovascular disease. With the use of (non-invasive) ultrasound techniques, arterial wall characteristics such as common carotid intima-media thickness (CIMT) can be evaluated. Numerous studies have now shown that these non-invasive measures of CIMT can be measured with high reproducibility; correlate well with major cardiovascular risk factors, prevalent cardiovascular disease and severity of atherosclerosis in other vascular beds; predict incident cardiovascular events; and that CIMT progression over time may be decreased by targeted interventions. As such, CIMT is regarded a suitable surrogate marker for cardiovascular disease and is currently widely used in clinical and epidemiological studies. However, methodological heterogeneity accounts for individual variability in the values of CIMT across studies. As such, values obtained in either research or clinical practice settings obtained with different methodologies are difficult to interpret and/or analyze in the absence of standardized reference values. 
In the next Chapter (Chapter 5) we therefore provide reference intervals for CIMT, which are based on a healthy sub-sample of combined subject-level data on common CIMT as obtained by echotracking systems from different study centres worldwide. In addition, we assessed the relation of cardiovascular risk factors with CIMT to enable comparison of CIMT values across (patient) groups with different cardiovascular risk profiles, helping interpretation of such measures obtained both in research and clinical settings.

In the following chapters, we then investigated associations between measures of the glycation pathway and cardiovascular disease and surrogate markers thereof such as the well-established CIMT. Chapter 6 and 7 describe the results on genetic variation in the GLO1 and RAGE gene, respectively, and Chapter 8 on circulating levels of advanced glycation endproducts. 



\section{CHAPTER 5}

\section{REFERENCE INTERYALS FOR COMMON CAROTID INTIMA-MEDIA THICKMESS MEASURED WITH ECHOTRACXINS - RELATION WITH RISK FACTORS}

LIAM ENSELEM, ISABEL FERREIRA, COEM dA STEHOUWER, PIERRE BOUTOUYRIE. STEPHAKE LAUREKT

ON BEHALF OF THE REFEREMCE VALUES FOR ARTERIAL MEASUREMEMTS COLLABORATION ${ }^{X}$ COMPLETE AUTHOR LIST IS INCLUDED IN THE APPEKDIX (TABLE A5.1) 


\section{ABSTRACT}

\section{Background}

Common carotid artery intima-media thickness (CIMT) is widely used as a surrogate marker of atherosclerosis, given its predictive association with cardiovascular disease (CVD). The interpretation of CIMT values has been hampered by the absence of reference values, however. We therefore aimed to establish reference intervals of CIMT, to help interpretation of these measures.

\section{Methods}

We combined common CIMT data, obtained by echotracking, on 24,871 individuals (53\% men; age range 15-101y) from 24 research centres worldwide. Individuals without CVD, cardiovascular risk factors (CV-RFs) and BP-, lipid- and/or glucose-lowering medication constituted a healthy sub-population $(n=4,234)$ used to establish sex-specific equations for percentiles of common CIMT across age. With these equations we generated CIMT Zscores in different reference sub-populations, thereby allowing for a standardized comparison between observed and predicted ('normal') values from individuals of the same age and sex.

\section{Results}

In the sub-population without CVD and treatment $(n=14,609)$, and in men and women respectively, CIMT Z-scores were independently influenced by SBP [standardized $\beta$ s 0.19 (95\% Cl: $0.16 ; 0.22)$ and $0.18(0.15 ; 0.21)]$, smoking [0.25 (0.19;0.31) and $0.11(0.04 ; 0.18)]$, diabetes $[0.19(0.05 ; 0.33)$ and $0.19(0.02 ; 0.36)]$, total-to-HDL cholesterol ratio [0.07 $(0.04 ; 0.10)$ and $0.05(0.02 ; 0.09)]$, and BMI [0.14 (0.12;0.17) and $0.07(0.04 ; 0.10)]$.

\section{Conclusion}

We estimated age- and sex-specific percentiles of common CIMT in a healthy population and assessed the relation of CV-RFs with CIMT Z-scores, which enables comparison of CIMT values for (patient) groups with different cardiovascular risk profiles, helping interpretation of such measures obtained both in research and clinical settings. 


\section{INTRODUCTION}

In 1986, Pignoli et al showed for the first time that measures, by means of ultrasonography, of the intima-media thickness of the common carotid artery (CIMT) did not differ significantly from CIMT measured by microscopic examination of the same specimens. This cornerstone observation lead the investigators to suggest that ultrasound imaging could represent a valuable non-invasive tool for the assessment of arterial wall thickness in vivo. ${ }^{1}$ Since then, numerous studies have shown that non-invasive measures of CIMT can be measured with high reproducibility; correlate well with major cardiovascular risk factors (CV-RFs), prevalent disease and severity of atherosclerosis in other vascular beds; predict incident cardiovascular events; and that its progression over time may be decreased by targeted interventions (reviewed $\mathrm{in}^{2-4}$ ). As such, CIMT is a suitable surrogate marker for cardiovascular disease (CVD) ${ }^{4}$ and is currently widely used for the pre-symptomatic detection of disease and its progression, in observational studies and clinical trials, improving efficiency and aiming at decreasing follow-up time usually needed in studies with 'hard' cardiovascular endpoints. ${ }^{4-7}$

The variability of CIMT readings at the population level depends primarily on age and sex $^{8}$ but is also influenced by several other CV-RFs, such as blood pressure (BP), smoking, diabetes, cholesterol levels and body fatness. ${ }^{9-12}$ Despite attempts at normalisation, the method for measuring CIMT is highly variable, either in terms of signal processing [(image analysis, radiofrequency (RF) signal analysis] or anatomical location. The methodological heterogeneity accounts for individual variability in the values of CIMT across studies. As such, values obtained in either research or clinical practice settings, obtained with different methodology, are difficult to analyze in the absence of standardized reference values. Indeed, although age-dependent nomograms for common CIMT have been reported before, ${ }^{8,13-16}$ their general use is limited because 1) they refer to mere distributions of mean or median values in general populations without distinguishing between subjects with or without established CV-RFs and/or disease ${ }^{8,14}$ and can thus not be used as reference for a 'normal' (i.e. healthy) population; and/or 2) they refer to values of common CIMT as obtained by manual or automated analyses techniques of B-mode ultrasound imaging, ${ }^{8,13-16}$ whereas, at present, automated edge-detection on the basis of RF signal processing (hereafter as 'echotracking') of B+M mode ultrasound imaging is probably the most accurate method ${ }^{17-19}$ with the advantage of enabling simultaneous measurement of common CIMT, diameter and distension for detailed mechanistic studies, $^{20-25}$ and/or 3 ) they are confined to a single-centre and/or country ${ }^{13,15,16}$ and thus have limited sample sizes to properly cover the whole (adult) age-range. 
In view of these considerations, we combined subject-level data on established CV-RFs and common CIMT as obtained by echotracking systems from different study centres worldwide into one large dataset - The Reference Values for Arterial Measurements Collaboration's CIMT database. This was used to, first, establish age- and sex-specific percentiles (reference intervals - RIs) for common CIMT in individuals without CV-RFs (as conventionally defined), prior CVD and BP-, lipid- and/or glucose-lowering medication - i.e. a healthy population; and second, to investigate the relation of CV-RFs and use of BP-, lipid- and/or glucose-lowering medication with common CIMT percentiles in individuals with or without prior CVD.

\section{METHODS}

\section{STUDY POPULATION}

With a systematic literature review we identified all cohort studies using echotracking for common CIMT measurement. Next, we personally contacted the principal investigators of the cohorts $(n=55)$ to inform them about the project and inviting them to participate. We finally compiled subject-level data from 24 research centres/research groups (of whom researchers accepted the invitation and provided data) - corresponding to 30 distinct cohorts - distributed across 14 countries worldwide. The contributing centres and the number of individuals included per centre/study are listed in the Appendix (Table A5.2). A total of 25,166 individuals with data on common CIMT obtained using echotracking systems, age [range 5-101 years], sex (13,430 men/11,736 women), CVD status, and important CV-RFs were available and registered in the total Reference Values for Arterial Measurements Collaboration's CIMT database. For the present study we excluded 295 (53\% girls) individuals who were aged $<15$ years because their data lacked sufficient variability with age (primarily concentrated at the age of five ${ }^{26}$ ) leaving 24,871 (47\% women) individuals for analyses.

To generate age- and sex-specific normative tables for common CIMT, we selected a healthy sub-population composed of individuals who did not meet any of the following criteria: 1) history of CVD; 2) use of BP-, lipid- and/or glucose-lowering medication; 3) hypertension [i.e. systolic blood pressure (SBP) $\geq 140 \mathrm{~mm} \mathrm{Hg}$ and/or diastolic blood pressure (DBP) $\geq 90 \mathrm{~mm} \mathrm{Hg}$ ] ${ }^{27} 4$ ) current smoking; 5) diabetes [defined as self-reported diabetes and/or fasting plasma glucose $\geq 7.0 \mathrm{mmol} / \mathrm{L}$ (if available) and/or postload plasma glucose $\geq 11.0 \mathrm{mmol} / \mathrm{L}$ (if available) ; $^{28} 6$ ) total cholesterol $>6.2 \mathrm{mmol} / \mathrm{L}^{29}$ 7) HDL cholesterol $<1.17 \mathrm{mmol} / \mathrm{L}$ (for men) and $<1.30 \mathrm{mmol} / \mathrm{L}$ (for women); ${ }^{29}$ and 8 ) body mass index $(\mathrm{BMI}) \geq 30 \mathrm{~kg} / \mathrm{m}^{2}$. ${ }^{30}$ This healthy sub-population consisted of $4,234(53 \%$ 
women) individuals, which originated from 21 out of the 24 research centres (the numbers in the healthy sub-population originating from each centre are given in Table A5.2 in the Appendix).

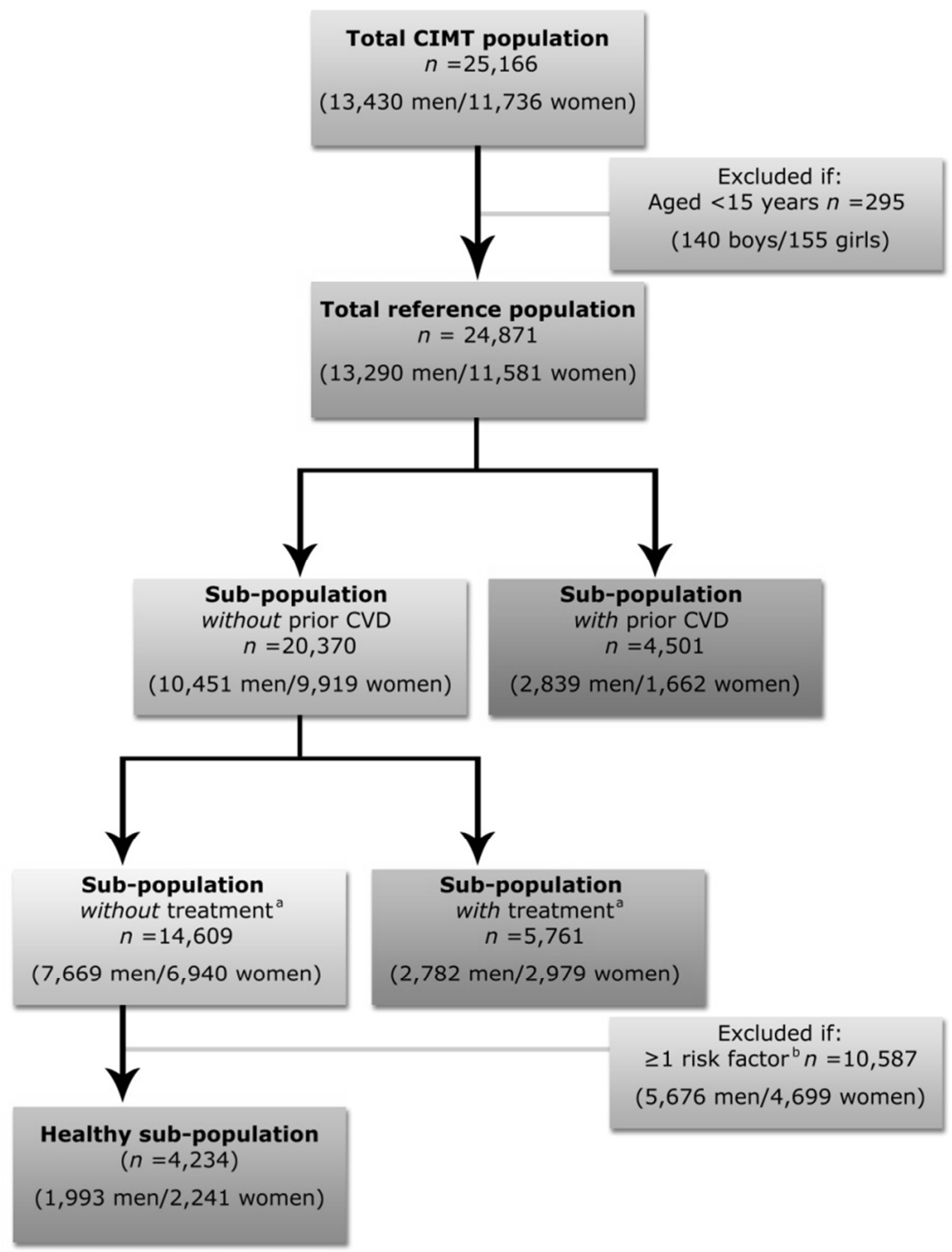

Figure 5.1 Study flowchart describing the selection and categorization of individuals from the total CIMT population to the reference and healthy sub-populations. ${ }^{\mathrm{a} B P}$-, lipid- and/or glucose-lowering medication; ${ }^{\mathrm{b}}$ Risk factors considered were hypertension (SBP/DBP $\geq 140 / 90 \mathrm{~mm} \mathrm{Hg}$ ), current smoking, diabetes [self-reported diabetes and/or fasting plasma glucose $\geq 7.0$ and/or postload plasma glucose $\geq 11.0 \mathrm{mmol} / \mathrm{L}$ (if available)], total cholesterol $>6.2 \mathrm{mmol} / \mathrm{L}, \mathrm{HDL}$ cholesterol $<1.17 \mathrm{mmol} / \mathrm{L}$ (for men) and $<1.30 \mathrm{mmol} / \mathrm{L}$ (for women), and $\mathrm{BMI} \geq 30 \mathrm{~kg} / \mathrm{m}^{2}$. 
To investigate the relation of CV-RFs with individuals' levels of common CIMT percentiles we stratified the total population according to history of CVD and, in individuals without prior CVD only, by use of BP-, lipid- and/or glucose-lowering medication. This resulted in three reference sub-populations consisting of: 1) 14,609 (48\% women) individuals without prior CVD and without use of BP-, lipid- and/or glucose-lowering medication; 2) 5,761 (52\% women) individuals without prior CVD and who used BP-, lipid- and/or glucoselowering medication; and 3) 4,501 individuals (37\% women) with prior CVD irrespective of medication use.

A flowchart describing the selection of the healthy and reference sub-populations and exact numbers per sex is presented in Figure 5.1.

\section{COMMON CIMT MEASUREMENTS - METHODOLOGICAL CONSIDERATIONS}

We only included common CIMT data obtained by means of pure echotracking or related techniques. These were measured at the far wall of the right and/or left common carotid artery only, because near wall readings from echotracking devices may not reflect true thickness and are thus seldom obtained. Mean of right and left common CIMT readings (if both sides were assessed) were used in the analyses, as previous studies have reported no differences between sides. ${ }^{14,31}$

Different types of ultrasound systems were used across centres; specifically, pure echotracking systems: the Wall Track System ( $n=13,116$; WTS, ESAOTE, Maastricht, the Netherlands ${ }^{32}$ ) and the ART.LAB system ( $n=8,519$; advanced version of WTS; ESAOTE, Maastricht, the Netherlands) or related techniques, which were validated against echotracking: the Vivid-7 ultrasound (US) system ( $n=2,524$; GE Vingmed Ultrasound, Horten, Norway) with Matlab post-processing (Institute of Biomedical Technology, Ghent University, Ghent, Belgium); ${ }^{33}$ the Aloka SSD-650 US system ( $n=606$; Aloka, Tokyo, Japan) with dedicated post-processing software (M'ATHS, Metris, France) ${ }^{34}$ and the Carotid Studio ( $n=401$; Institute of Clinical Physiology, National Research Council, Pisa, Italy). ${ }^{35}$ The exact anatomical location of the measurement of the common CIMT also differed across centres: i.e., at $0-1 \mathrm{~cm}$, centred at $1 \mathrm{~cm}$, at $1-2 \mathrm{~cm}$ or centred at $2 \mathrm{~cm}$ proximal to the carotid bifurcation. Therefore, prior to further analyses, we first standardized all common CIMT values obtained with different echotracking systems and anatomical locations (for details on echotracking system and anatomical location of measurement by each study please see Appendix Table A5.2). To this aim, original common CIMT values were rescaled to the same metric of the mostly used system and location, i.e. measurements with the ART.LAB system and centred at $1 \mathrm{~cm}$ proximal to the carotid bifurcation (please see details on standardization procedures in the section 'statistical analyses' below). 


\section{STATISTICAL ANALYSES}

Multiple imputation of missing values in variables.

A total of 4,673 individuals (19\% of the total reference population) had missing values for one $(n=4,391)$ or more $(n=282)$ of the variables of interest. The percentage of missing values per variable varied from $0.4 \%$ (current smoking) to $11 \%$ (HDL cholesterol). We used multiple imputation chained equations to impute those values rather than perform complete case analyses in order to decrease bias and increase the power of the analyses. ${ }^{36,37}$ With this procedure the imputation model of a single missing variable uses the information from all the other variables available in the dataset (i.e. centre, age, body mass, height, SBP, DBP, smoking status, diabetes, total and HDL-cholesterol, use of BP-, lipid and/or glucose-lowering medication and common CIMT) as predictors by appropriate regression models (i.e. linear or logistic if variable to be imputed was continuous or dichotomous, respectively). We generated five imputed datasets, accounting for design features such as clustering within centres and stratification by sex and history of CVD. The imputed datasets were used to fit the regression models of interest and results reported were those retrieved from pooled analyses on all five imputed datasets.

\section{Standardization of CIMT measurements.}

For the purpose of technique standardization, we performed multiple linear regression analyses that included dummy variables for each echotracking system (with ART.LAB as reference) and anatomical location (with measurements centred at $1 \mathrm{~cm}$ proximal to the carotid bifurcation as reference) as independent determinants of common CIMT. These analyses were conducted in the total population $(n=24,871)$ and included adjustments for all CV-RFs (i.e. age, sex, SBP, smoking, diabetes, total and HDL cholesterol, BMI, history of CVD and use of BP- and/or lipid-lowering medication). The regression coefficients ( $\beta$ ) for the dummy variables hereby obtained showed that, compared with the ART.LAB system, all other echotracking systems had slightly higher common CIMT values, and the more distal to the carotid bifurcation measurements were taken, the lower the common CIMT value was (Table A5.3 - Appendix). These regression coefficients were used as 'calibration factors' to rescale individual common CIMT values to the reference technique. We used these rescaled common CIMT values in all further analyses.

Definition of age- and sex-specific reference intervals.

RIs, also commonly referred to as 'normal ranges', are routinely used in medicine to determine whether values from a given clinical measure are 'normal' or 'abnormal'. An RI is defined by the range between two percentile values centred about the median on the probability scale, preferably in a population of individuals who satisfy well-defined criteria of health. Standard limits commonly used are the $2.5^{\text {th }}$ and the $97.5^{\text {th }}$ percentiles, which correspond to a $95 \% \mathrm{RI}$, but others may also be of interest. We prefer the term 'reference interval' rather than 'normal range' since the latter implies that any observation outside the range indicates 'abnormality or disease', which may not always be the case. 
Conversely, observations inside the range (e.g. $>75^{\text {th }}$ percentile) may already indicate increased cardiovascular risk. ${ }^{38}$

Calculation of age-specific RIs for common CIMT was conducted in the healthy sub-population $(n=4,234)$, and in men and women separately. To this aim we used a parametric regression method based on fractional polynomials (FPs) as described by Royston and Wright ${ }^{38,39}$ and implemented in STATA software (version 11.0 Stata Corp., College Station, TX, USA). ${ }^{40}$ Briefly, common CIMT data were assumed to be normally distributed, conditional on age. With the STATA command xrig/s, the best fitting FPs for the age-specific mean and standard deviation (SD) regression curves were defined using an iterative procedure (generalised least squares - GLS). ${ }^{40}$ Results of these analyses enable estimation of the age-specific mean and SD of CIMT as mean CIMT $=a+b^{*} a g e^{p}+c^{*} a g e^{q}+\ldots$, where $a, b, c, \ldots$ are the coefficients and $p, q, \ldots$ are the powers with numbers selected from the set $[-2,-1,-0.5,0,0.5,1,2,3]$ estimated from the regression for the mean ${ }_{C I M T}$ curve, and likewise from the regression for the $S D_{C I M T}$ curve. For example, FPs with powers [1 2], that is, with $p=1$ and $q=2$, illustrate an equation with the form $a+b^{*}$ age $+c^{*}$ age ${ }^{2} .^{39}$ Estimated mean, $S D$ and Z-scores [i.e., (observed $d_{\mathrm{CIMT}}-$ mean $_{\mathrm{CIMT}}$ ) $/ \mathrm{SD}_{\mathrm{CIMT}}$ ] were all stored in the dataset. The Z-scores were used to assess the model fit, which was deemed appropriate if these were normally distributed with a mean of 0 and an SD of 1 , and randomly scattered above and below 0 when plotted against age. Finally, age-specific $2.5^{\text {th }}, 10^{\text {th }}, 25^{\text {th }}, 50^{\text {th }}, 75^{\text {th }}, 90^{\text {th }}$, and $97.5^{\text {th }}$ percentile curves were calculated as mean CIMT + $Z_{p}{ }^{*} S D$, where $Z_{p}$ assumed the values of $-1.96,-1.28,-0.67,0,0.67,1.28$, and 1.96, respectively.

Relation with risk factors.

Based on the equations estimated as described above, we computed expected 'normal' mean common CIMT values for each individual in the reference sub-populations (i.e. those with and without CVD and/or medication) and calculated age- and sex-specific common CIMT Z-scores as (observed ${ }_{\mathrm{CIMT}}$ - expected $_{\mathrm{CIMT}}$ )/SDexpected $\mathrm{SIMT}_{\mathrm{CIMT}}$; this allows for a standardized comparison between observed common CIMT values vs. those from healthy individuals of the same age and sex, expressed by the number of SDs an individual measurement lies above or below the healthy population mean (or $50^{\text {th }}$ percentile).

The relation of known CV-RFs with the common CIMT Z-scores was then investigated in the different reference sub-populations using multiple linear regression analyses to enable interpretation of common CIMT values across different risk groups. We also included age in these analyses to account for any potential residual influence of age in these sub-populations.

Statistical analyses were performed using the Predictive Analytics SoftWare, version 18.0 (SPSS IBM Corporation, Armonk, NY, USA) unless specified otherwise. 
Table 5.1 Risk factors and clinical characteristics of the total, healthy and reference sub-populations in men

\begin{tabular}{|c|c|c|c|c|c|}
\hline \multirow[b]{3}{*}{$n$} & \multirow{3}{*}{$\begin{array}{c}\text { Total Reference } \\
\text { population } \\
13,290\end{array}$} & \multirow{3}{*}{$\begin{array}{c}\text { Healthy } \\
\text { sub-population } \\
1,993\end{array}$} & \multicolumn{2}{|c|}{ Sub-population without CVD } & \multirow{3}{*}{$\begin{array}{c}\text { Sub-population } \\
\text { with CVD } \\
2,839\end{array}$} \\
\hline & & & without treatment $^{\mathrm{a}}$ & with treatment ${ }^{a}$ & \\
\hline & & & 7,669 & 2,782 & \\
\hline Age [years (range)] & $56(15-99)$ & $50(15-90)$ & $53(15-99)$ & $59(16-98)$ & $63(23-97)$ \\
\hline Body mass index $\left(\mathrm{kg} / \mathrm{m}^{2}\right)$ & $26.3 \pm 3.7$ & $24.2 \pm 2.6$ & $25.8 \pm 3.5$ & $27.6 \pm 3.9$ & $26.6 \pm 3.5$ \\
\hline Systolic BP (mm Hg) & $136 \pm 19$ & $123 \pm 10$ & $132 \pm 17$ & $142 \pm 19$ & $139 \pm 20$ \\
\hline Diastolic BP (mm Hg) & $79 \pm 11$ & $74 \pm 8$ & $79 \pm 11$ & $82 \pm 12$ & $79 \pm 11$ \\
\hline Mean arterial pressure $(\mathrm{mm} \mathrm{Hg})$ & $98 \pm 12$ & $90 \pm 8$ & $96 \pm 12$ & $102 \pm 13$ & $99 \pm 12$ \\
\hline Hypertension [n (\%)] & $6,951(52)$ & - & $2,453(32)$ & $2,324(84)$ & $2,173(77)$ \\
\hline Total cholesterol (mmol/L) & $5.5 \pm 1.0$ & $5.1 \pm 0.7$ & $5.5 \pm 1.0$ & $5.4 \pm 1.1$ & $5.3 \pm 1.0$ \\
\hline LDL cholesterol (mmol/L) & $3.5 \pm 0.9$ & $3.1 \pm 0.7$ & $3.6 \pm 0.9$ & $3.4 \pm 1.0$ & $3.3 \pm 0.9$ \\
\hline HDL cholesterol (mmol/L) & $1.3 \pm 0.3$ & $1.5 \pm 0.3$ & $1.3 \pm 0.3$ & $1.3 \pm 0.3$ & $1.2 \pm 0.3$ \\
\hline Total-to-HDL cholesterol ratio & $4.5 \pm 1.5$ & $3.4 \pm 0.7$ & $4.4 \pm 1.4$ & $4.6 \pm 1.7$ & $4.6 \pm 1.6$ \\
\hline Triglycerides (mmol/L) & $1.3(0.9-1.9)$ & $0.9(0.7-1.2)$ & $1.2(0.9-1.8)$ & $1.4(1.0-2.0)$ & $1.4(1.0-2.0)$ \\
\hline Fasting glucose (mmol/L) & $5.8 \pm 1.5$ & $5.2 \pm 0.7$ & $5.4 \pm 1.0$ & $6.2 \pm 2.0$ & $6.2 \pm 1.8$ \\
\hline Diabetes [n (\%)] & $1,408(11)$ & - & $304(4)$ & $590(21)$ & $514(18)$ \\
\hline Current smoking [n (\%)] & $3,126(24)$ & - & $1,825(24)$ & $559(20)$ & $741(26)$ \\
\hline BP-lowering medication [n (\%)] & $2,335(18)$ & - & - & $2,073(74)$ & $1,648(58)$ \\
\hline Lipid-lowering medication [n (\%)] & $877(7)$ & - & - & $1,134(41)$ & $1,129(40)$ \\
\hline Glucose-lowering medication [n (\%)] & $595(5)$ & - & - & $379(14)$ & $216(8)$ \\
\hline History of CVD [n (\%)] & $2,839(21)$ & - & - & - & $2,839(100)$ \\
\hline Common CIMT $(\mu \mathrm{m})$ & $653 \pm 159$ & $583 \pm 131$ & $631 \pm 155$ & $682 \pm 151$ & $685 \pm 169$ \\
\hline
\end{tabular}

Data are presented as means \pm SD, medians [interquartile ranges] or numbers (percentages), as appropriate. ${ }^{a} \mathrm{BP}$-, lipid- and glucose-lowering treatment. 
Table 5.2 Risk factors and clinical characteristics of the total, healthy and reference sub-populations in women

\begin{tabular}{|c|c|c|c|c|c|}
\hline \multirow[b]{3}{*}{$n$} & \multirow{3}{*}{$\begin{array}{c}\text { Total Reference } \\
\text { population } \\
11,581\end{array}$} & \multirow{3}{*}{$\begin{array}{c}\text { Healthy } \\
\text { sub-population } \\
2,241\end{array}$} & \multicolumn{2}{|c|}{ Sub-population without CVD } & \multirow{3}{*}{$\begin{array}{c}\text { Sub-population } \\
\text { with CVD } \\
1,662\end{array}$} \\
\hline & & & without treatment $^{\mathrm{a}}$ & with treatment ${ }^{a}$ & \\
\hline & & & 6,940 & 2,979 & \\
\hline Age [years (range)] & $58(15-101)$ & $48(15-89)$ & $54(15-95)$ & $63(17-101)$ & $64(20-95)$ \\
\hline Body mass index $\left(\mathrm{kg} / \mathrm{m}^{2}\right)$ & $25.8 \pm 4.7$ & $22.9 \pm 2.8$ & $24.9 \pm 4.3$ & $27.8 \pm 5.0$ & $26.4 \pm 4.5$ \\
\hline Systolic BP (mm Hg) & $133 \pm 21$ & $118 \pm 11$ & $128 \pm 19$ & $142 \pm 21$ & $139 \pm 21$ \\
\hline Diastolic BP $(\mathrm{mm} \mathrm{Hg})$ & $76 \pm 11$ & $72 \pm 8$ & $75 \pm 10$ & $79 \pm 12$ & $77 \pm 11$ \\
\hline Mean arterial pressure $(\mathrm{mm} \mathrm{Hg})$ & $95 \pm 13$ & $87 \pm 8$ & $93 \pm 12$ & $100 \pm 13$ & $98 \pm 12$ \\
\hline Hypertension [n (\%)] & $5,519(48)$ & - & $1,804(26)$ & $2,608(88)$ & $1,106(66)$ \\
\hline Total cholesterol (mmol/L) & $5.8 \pm 1.1$ & $5.2 \pm 0.7$ & $5.8 \pm 1.1$ & $5.9 \pm 1.1$ & $5.9 \pm 1.1$ \\
\hline LDL cholesterol (mmol/L) & $3.6 \pm 1.0$ & $3.0 \pm 0.7$ & $3.6 \pm 1.0$ & $3.6 \pm 1.0$ & $3.7 \pm 1.0$ \\
\hline HDL cholesterol (mmol/L) & $1.6 \pm 0.4$ & $1.8 \pm 0.3$ & $1.7 \pm 0.4$ & $1.5 \pm 0.4$ & $1.6 \pm 0.4$ \\
\hline Total-to-HDL cholesterol ratio & $3.8 \pm 1.3$ & $2.9 \pm 0.6$ & $3.7 \pm 1.2$ & $4.1 \pm 1.4$ & $4.1 \pm 1.4$ \\
\hline Triglycerides (mmol/L) & $1.2(0.8-1.6)$ & $0.9(0.7-1.1)$ & $1.1(0.8-1.5)$ & $1.4(1.0-1.9)$ & $1.3(0.9-1.8)$ \\
\hline Fasting glucose (mmol/L) & $5.6 \pm 1.4$ & $4.9 \pm 0.6$ & $5.2 \pm 0.9$ & $6.0 \pm 1.8$ & $5.9 \pm 1.7$ \\
\hline Diabetes [n (\%)] & $981(9)$ & - & $210(3)$ & $505(17)$ & $266(16)$ \\
\hline Current smoking [n (\%)] & $1,983(17)$ & - & $1,237(18)$ & $433(15)$ & $313(19)$ \\
\hline BP-lowering medication [n (\%)] & $2,378(21)$ & - & - & $2,405(81)$ & $767(46)$ \\
\hline Lipid-lowering medication [n (\%)] & $618(5)$ & - & - & $991(33)$ & $421(25)$ \\
\hline Glucose-lowering medication [n (\%)] & $356(3)$ & - & - & $260(9)$ & $96(6)$ \\
\hline History of CVD [n (\%)] & $1,662(14)$ & - & - & - & $1,662(100)$ \\
\hline Common CIMT $(\mu \mathrm{m})$ & $639 \pm 148$ & $561 \pm 123$ & $610 \pm 140$ & $682 \pm 150$ & $677 \pm 153$ \\
\hline
\end{tabular}

Data are presented as means \pm SD, medians [interquartile ranges] or numbers (percentages), as appropriate. aBP-, lipid- and glucose-lowering treatment. 


\section{RESULTS}

Tables 5.1 and 5.2 show the participants' characteristics of the total, healthy and reference sub-populations, in men and women, respectively.

\section{AGE- AND SEX-SPECIFIC REFERENCE INTERVALS FOR COMMON CIMT}

In analyses confined to the healthy sub-population, the best fitting FPs' powers $(p)$ for the mean $_{\mathrm{CIMT}}$ and $\mathrm{SD}_{\mathrm{CIMT}}$ curves were $p=1$ for both men and women, indicating that linear regression lines described the age-CIMT relationship well. Accordingly, the equations derived on the basis of the estimated coefficients were, for men:

- $\operatorname{mean}_{\text {CIMT }}($ in $\mu \mathrm{m})=323.5+5.201 *$ age

- $S D_{\text {CIMT }}($ in $\mu \mathrm{m})=57.24+0.9027^{*}$ age

and, for women:

- $\operatorname{mean}_{\mathrm{CIMT}}$ (in $\left.\mu \mathrm{m}\right)=321.7+4.971 *$ age

- $S D_{\text {CIMT }}$ (in $\left.\mu \mathrm{m}\right)=54.50+0.8256^{*}$ age .

The estimated Z-scores had a mean value of 0 and an SD of 1 and, when plotted against age, were randomly distributed above and below 0 (Figure A5.1 - Appendix), indicating good model fit and no residual dependency on age.

Sex-specific percentile lines superimposed on the raw data are shown in Figure 5.2 and the respective levels of common CIMT by age category are presented in Table 5.3. Mean values of common CIMT were slightly higher in men than in women at any age $(p<0.001)$ but increases in common CIMT with aging were similar in men $(5.2 \mu \mathrm{m} /$ year $)$ and women $(5.0 \mu \mathrm{m} /$ year) ( $p$ for age by sex interaction $=0.144)$.

\section{RELATION OF CV-RFS WITH COMMON CIMT PERCENTILES AS DEFINED IN THE HEALTHY SUB-POPULATION}

In the sub-population without prior CVD and treatment, and both in men and women, higher common CIMT Z-scores (i.e. positive deviation from the healthy population mean) were significantly related to SBP, smoking, diabetes, total-to-HDL cholesterol ratio and $\mathrm{BMI}$, whereas in the sub-population without prior CVD but under BP-, lipid- and/or glucose-lowering treatment, diabetes and total-to-HDL cholesterol ratio were no longer independent determinants of the common CIMT Z-scores (Table 5.4). In the subpopulation with prior CVD, SBP was the main determinant of common CIMT Z-scores in 
both men and women; BMI (adversely) and use of lipid-lowering medication (protectively) were also determinants but in men only.

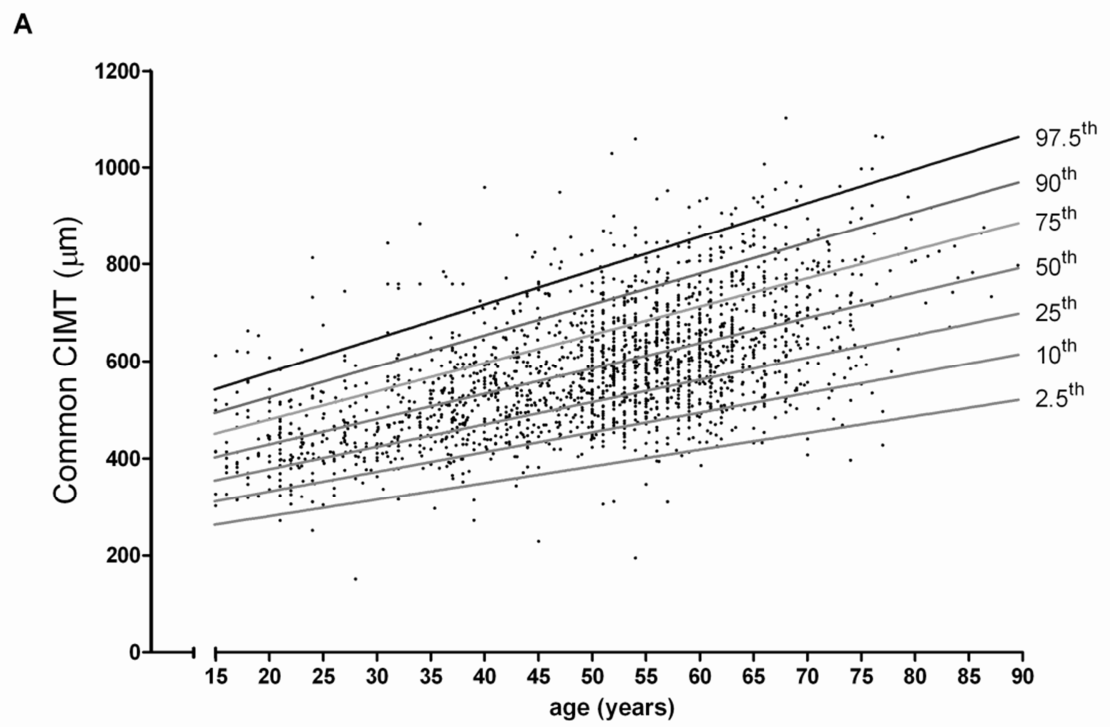

B

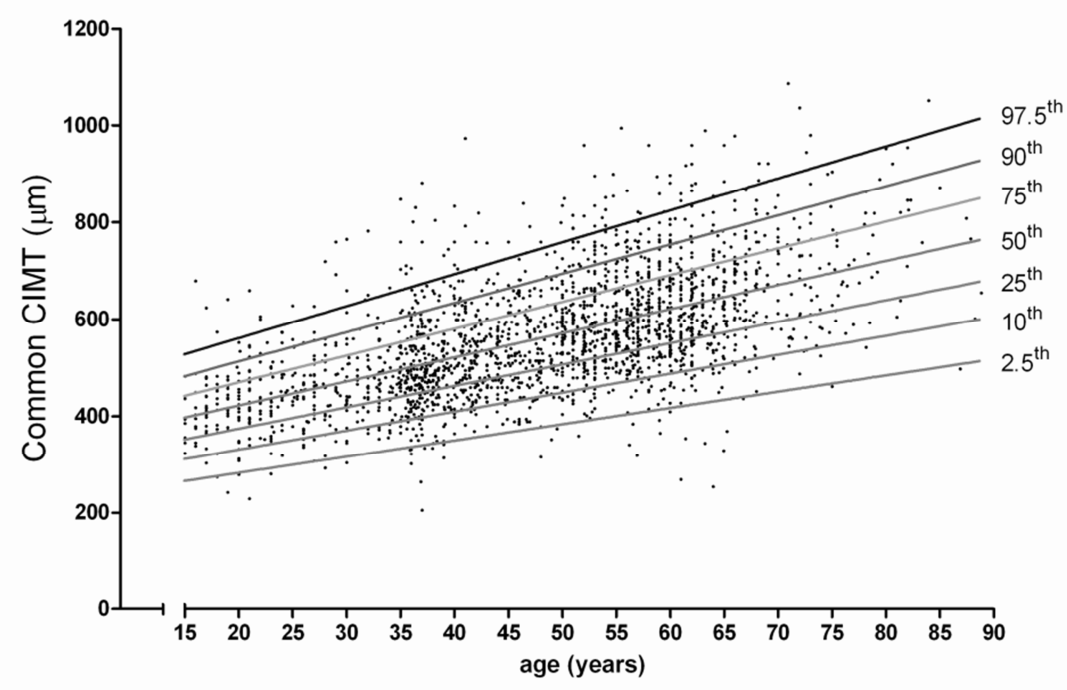

Figure 5.2 Age-specific percentiles of common CIMT in the healthy sub-population. $\boldsymbol{A}$, men; $\boldsymbol{B}$, women. 
Table 5.3 Age- and sex-specific percentiles of common CIMT (in $\mu \mathrm{m}$ ) in the healthy sub-population

\begin{tabular}{|c|c|c|c|c|c|c|c|c|}
\hline & \multirow[b]{2}{*}{ Age (years) } & \multicolumn{7}{|c|}{ percentiles } \\
\hline & & 2.5th & 10th & 25th & 50th & 75th & 90th & 97.5th \\
\hline \multirow[t]{15}{*}{ Men $(n=1,993)$} & 15 & 263 & 311 & 354 & 401 & 449 & 492 & 540 \\
\hline & 20 & 280 & 331 & 377 & 427 & 478 & 524 & 575 \\
\hline & 25 & 297 & 351 & 400 & 453 & 507 & 556 & 610 \\
\hline & 30 & 314 & 372 & 423 & 479 & 536 & 587 & 645 \\
\hline & 35 & 331 & 392 & 446 & 505 & 565 & 619 & 680 \\
\hline & 40 & 349 & 412 & 468 & 531 & 594 & 651 & 714 \\
\hline & 45 & 366 & 432 & 491 & 557 & 624 & 683 & 749 \\
\hline & 50 & 383 & 452 & 514 & 583 & 653 & 715 & 784 \\
\hline & 55 & 400 & 473 & 537 & 609 & 682 & 746 & 819 \\
\hline & 60 & 417 & 493 & 560 & 635 & 711 & 778 & 854 \\
\hline & 65 & 434 & 513 & 583 & 662 & 740 & 810 & 889 \\
\hline & 70 & 451 & 533 & 606 & 688 & 769 & 842 & 924 \\
\hline & 75 & 469 & 554 & 629 & 714 & 798 & 873 & 958 \\
\hline & 80 & 486 & 574 & 652 & 740 & 827 & 905 & 993 \\
\hline & 85 & 503 & 594 & 675 & 766 & 856 & 937 & 1028 \\
\hline \multirow[t]{15}{*}{ Women $(n=2,241)$} & 15 & 265 & 311 & 351 & 396 & 441 & 482 & 527 \\
\hline & 20 & 282 & 330 & 373 & 421 & 469 & 512 & 560 \\
\hline & 25 & 299 & 350 & 395 & 446 & 497 & 542 & 593 \\
\hline & 30 & 315 & 369 & 417 & 471 & 524 & 572 & 626 \\
\hline & 35 & 332 & 389 & 439 & 496 & 552 & 602 & 659 \\
\hline & 40 & 349 & 408 & 461 & 521 & 580 & 633 & 692 \\
\hline & 45 & 366 & 428 & 483 & 545 & 607 & 663 & 725 \\
\hline & 50 & 382 & 448 & 506 & 570 & 635 & 693 & 758 \\
\hline & 55 & 399 & 467 & 528 & 595 & 663 & 723 & 791 \\
\hline & 60 & 416 & 487 & 550 & 620 & 690 & 753 & 824 \\
\hline & 65 & 433 & 506 & 572 & 645 & 718 & 783 & 857 \\
\hline & 70 & 450 & 526 & 594 & 670 & 745 & 813 & 890 \\
\hline & 75 & 466 & 545 & 616 & 694 & 773 & 843 & 923 \\
\hline & 80 & 483 & 565 & 638 & 719 & 801 & 874 & 956 \\
\hline & 85 & 500 & 585 & 660 & 744 & 828 & 904 & 989 \\
\hline
\end{tabular}

To enable comparison of the strength of the associations between the individual CV-RFs and common CIMT Z-scores within each sub-population, these associations are also shown as standardized regression coefficients (i.e. per SD increase in CV-RF) - Figure 5.3. These analyses showed that, in the sub-population without CVD or treatment, smoking, diabetes and SBP were the strongest determinants of the common CIMT Z-scores in men, whereas in women these were diabetes and SBP; comparisons by sex showed that smoking and $\mathrm{BMI}$ were stronger determinants in men than in women ( $p$ for sex interactions were 0.005 and $<0.001$, respectively). 
Table 5.4 Relation of known cardiovascular risk factors with common CIMT Z-scores in the reference sub-populations

\begin{tabular}{|c|c|c|c|c|c|c|c|c|c|c|}
\hline \multirow[b]{3}{*}{ Sex } & \multirow[b]{3}{*}{ Risk factor } & \multicolumn{6}{|c|}{ Sub-population without CVD } & \multirow{2}{*}{\multicolumn{3}{|c|}{$\begin{array}{l}\text { Sub-population with CVD } \\
\qquad(n=4,501)\end{array}$}} \\
\hline & & \multicolumn{3}{|c|}{$\begin{array}{l}\text { without treatment }{ }^{\mathrm{a}} \\
(=14,609)\end{array}$} & \multicolumn{3}{|c|}{$\begin{array}{l}\text { with treatment } \\
\quad(n=5,761)\end{array}$} & & & \\
\hline & & B & $95 \% \mathrm{Cl}$ & $P$-value & B & $95 \% \mathrm{Cl}$ & P-value & B & $95 \% \mathrm{Cl}$ & $P$-value \\
\hline \multirow[t]{8}{*}{ Men } & Systolic pressure $(10 \mathrm{~mm} \mathrm{Hg})$ & 0.111 & $0.093 ; 0.128$ & $<0.001$ & 0.070 & $0.045 ; 0.094$ & $<0.001$ & 0.080 & $0.055 ; 0.106$ & $<0.001$ \\
\hline & Current smoking (yes) & 0.248 & $0.185 ; 0.312$ & $<0.001$ & 0.143 & $0.026 ; 0.259$ & 0.016 & 0.100 & $-0.013 ; 0.213$ & 0.082 \\
\hline & Diabetes (yes) & 0.189 & $0.046 ; 0.332$ & 0.010 & 0.056 & $-0.053 ; 0.166$ & 0.315 & 0.108 & $-0.055 ; 0.271$ & 0.192 \\
\hline & Total-to-HDL cholesterol ratio (unit) & 0.051 & $0.029 ; 0.073$ & $<0.001$ & 0.022 & $-0.006 ; 0.049$ & 0.118 & 0.008 & $-0.028 ; 0.044$ & 0.660 \\
\hline & Body mass index $\left(\mathrm{kg} / \mathrm{m}^{2}\right)$ & 0.041 & $0.033 ; 0.049$ & $<0.001$ & 0.036 & $0.024 ; 0.048$ & $<0.001$ & 0.028 & $0.013 ; 0.042$ & $<0.001$ \\
\hline & Use of BP-lowering medication (yes) & - & - & - & - & - & - & -0.059 & $-0.161 ; 0.044$ & 0.264 \\
\hline & Use of lipid-lowering medication (yes) & - & - & - & - & - & - & -0.182 & $-0.287 ;-0.077$ & 0.001 \\
\hline & Use of glucose-lowering medication (yes) & - & - & - & - & - & - & -0.137 & $-0.370 ; 0.096$ & 0.248 \\
\hline \multirow[t]{8}{*}{ Women } & Systolic pressure $(10 \mathrm{~mm} \mathrm{Hg})$ & 0.097 & $0.080 ; 0.113$ & $<0.001$ & 0.100 & $0.078 ; 0.123$ & $<0.001$ & 0.101 & $0.068 ; 0.134$ & $<0.001$ \\
\hline & Current smoking (yes) & 0.111 & $0.040 ; 0.181$ & 0.002 & 0.204 & $0.078 ; 0.331$ & 0.002 & -0.107 & $-0.269 ; 0.054$ & 0.192 \\
\hline & Diabetes (yes) & 0.189 & $0.020 ; 0.359$ & 0.029 & 0.010 & $-0.109 ; 0.128$ & 0.872 & 0.052 & $-0.161 ; 0.265$ & 0.633 \\
\hline & Total-to-HDL cholesterol ratio (unit) & 0.043 & $0.016 ; 0.069$ & 0.002 & 0.021 & $-0.016 ; 0.059$ & 0.260 & 0.016 & $-0.034 ; 0.065$ & 0.534 \\
\hline & Body mass index $\left(\mathrm{kg} / \mathrm{m}^{2}\right)$ & 0.017 & $0.010 ; 0.024$ & $<0.001$ & 0.010 & $0.000 ; 0.020$ & 0.040 & 0.007 & $-0.008 ; 0.022$ & 0.387 \\
\hline & Use of BP-lowering medication (yes) & - & - & - & - & - & - & 0.072 & $-0.062 ; 0.205$ & 0.292 \\
\hline & Use of lipid-lowering medication (yes) & - & - & - & - & - & - & -0.102 & $-0.250 ; 0.046$ & 0.178 \\
\hline & Use of glucose-lowering medication (yes) & - & - & - & - & - & - & 0.109 & $-0.209 ; 0.427$ & 0.501 \\
\hline
\end{tabular}

The regression coefficient $\beta$ represents the increase in common CIMT (in SD from the healthy population mean among individuals of the same age and sex) per unit increase in each risk factor. $\beta$ s were obtained from multivariable regression models including all risk factors and age. ${ }^{\mathrm{a}} \mathrm{BP}$-, lipid- and glucose-lowering treatment. 
A

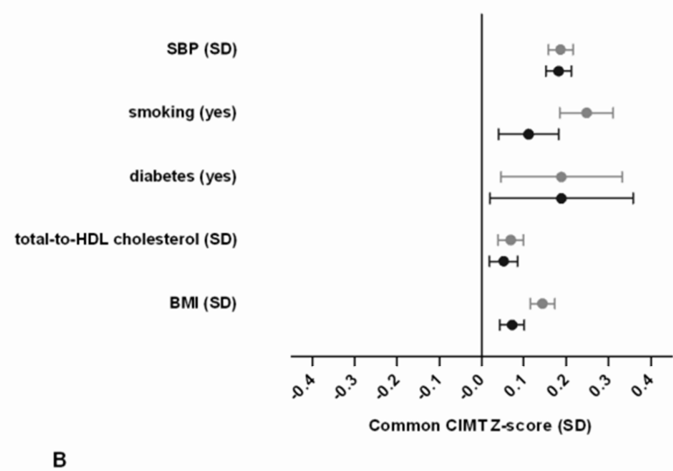

B

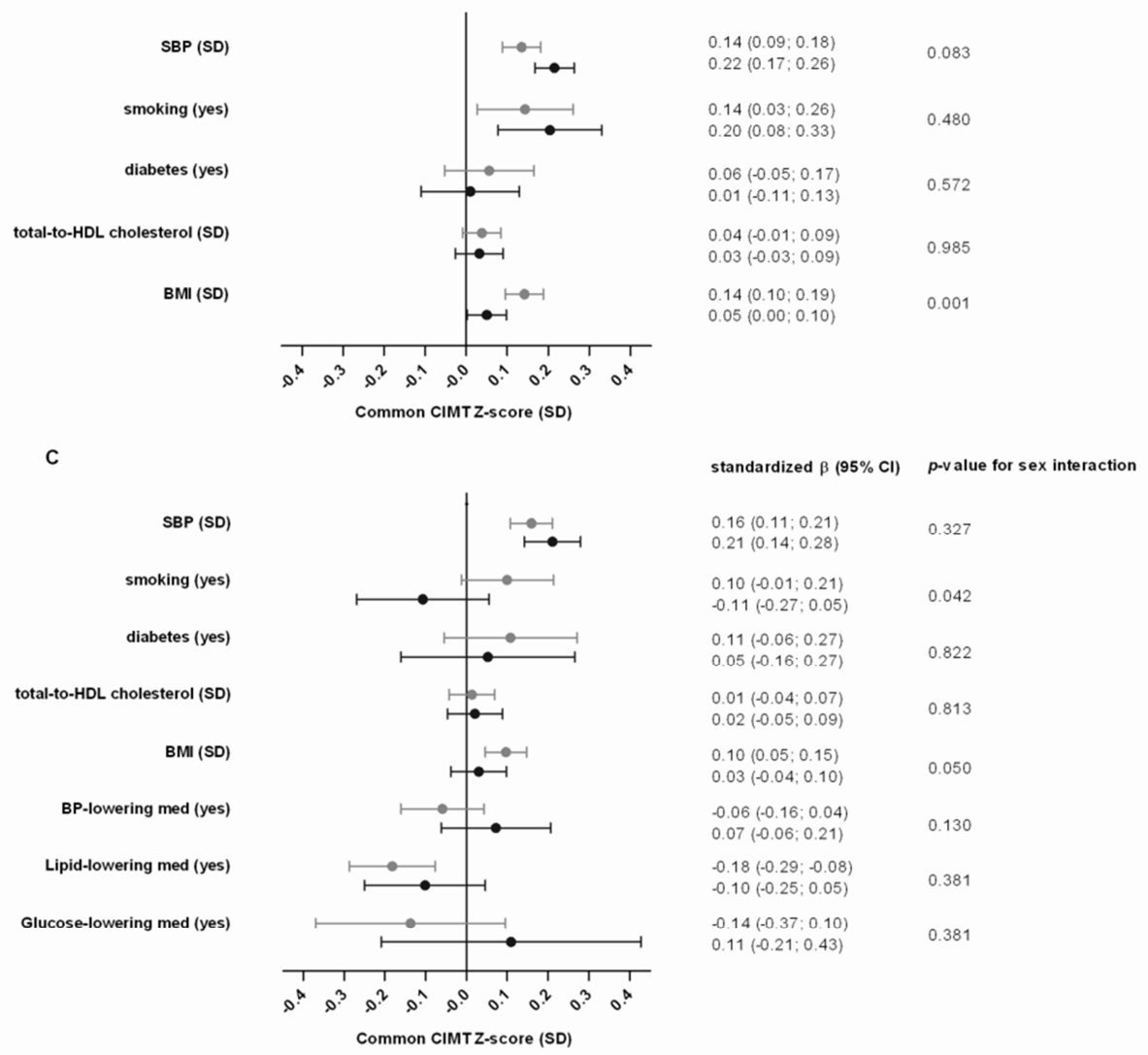

standardized $\beta(95 \% \mathrm{Cl}) \quad p$-v alue for sex interaction

$0.19(0.16 ; 0.22)$

$0.25(0.19 ; 0.31) \quad 0.005$

$0.11(0.04 ; 0.18)$

0.005

$0.07(0.04 ; 0.10) \quad 0.647$

$\begin{array}{ll}0.05(0.02 ; 0.09) & 0.647 \\ 0.14(0.12 ; 0.17) & <0.001\end{array}$
$0.19(0.05 ; 0.33) \quad 0.997$ standardized $\beta(95 \% \mathrm{Cl}) p$-v alue for sex interaction

$0.14(0.09 ; 0.18)$

$.14(0.03 ; 0.26)$

$0.20(0.08: 0.33)$

$06(-0.05 ; 0.17)$

$01(-0.11 ; 0.13)$

$.14(0.10 ; 0.19)$

standardized $\beta(95 \% \mathrm{Cl})$

n

$.03(-0.04: 0.10)$

$14(-0,37: 010)$

$11(-0.21 ; 0.43)$

Figure 5.3 Point estimates and 95\% confidence intervals represent the increase in common CIMT Z-score (in SD from the healthy population mean) per SD increase (or for presence vs. absence) in risk factor resulting from a multivariable regression model including all risk factors and age. Data in grey and black concern men and women, respectively.

$\boldsymbol{A}$, reference sub-population without CVD or treatment; $\boldsymbol{B}$, reference sub-population without CVD with BP-, lipidand/or glucose-lowering treatment; $\boldsymbol{C}$, reference sub-population with CVD. $B M I$, body mass index; $B P$, blood pressure; med, medication; SBP, systolic blood pressure. 
The regression coefficients shown in Table 5.4, reflecting the influence of CV-RFs on common CIMT Z-scores (i.e. the increase in SD from the mean common CIMT of healthy individuals of the same age and sex), can be converted into percentiles for a more meaningful interpretation of these analyses at the light of the RIs provided (see Figure 5.1 and Table 5.3). This is illustrated with two hypothetical subjects in Table 5.5: a 50-year old man with SBP $160 \mathrm{~mm} \mathrm{Hg}$, total-to-HDL cholesterol ratio 7.2, BMI $35 \mathrm{~kg} / \mathrm{m}^{2}$, who smokes and has no diabetes and a 50-year old woman with SBP $130 \mathrm{~mm} \mathrm{Hg}$, total-to-HDL cholesterol ratio $3.9, \mathrm{BMI} 24 \mathrm{~kg} / \mathrm{m}^{2}$, who does not smoke and has no diabetes. Based on these risk profiles and the regression coefficients provided, the estimated common CIMT Z-scores for these individuals were 1.32 (man) and 0.19 (woman); these correspond, respectively, to the $91^{\text {st }}$ and $58^{\text {th }}$ percentiles of the common CIMT distribution in individuals of the same age and sex from the healthy sub-population. Similarly, common CIMT Z-scores can be estimated for any other combination of individuals' age and CV-RFs and conversion into percentiles can easily be retrieved using any Standard Normal Distribution (Z) Table.

Table 5.5 Two examples of hypothetical subjects and their estimated common CIMT percentile

\begin{tabular}{|c|c|c|c|c|}
\hline & Cardiovascular risk factors & Coefficient $^{a}$ & Observed $^{b}$ & Coefficient * Observed \\
\hline \multirow[t]{9}{*}{ Man } & Intercept $=-2.604^{\mathrm{C}}$ & & & \\
\hline & Age $(10 \text { years })^{d}$ & 0.020 & 50 & $0.100^{f}$ \\
\hline & SBP $(10 \mathrm{~mm} \mathrm{Hg})$ & 0.111 & 160 & $1.776^{f}$ \\
\hline & Smoking (yes) & 0.248 & Yes (1) & 0.248 \\
\hline & Diabetes (yes) & 0.189 & No $(0)$ & 0 \\
\hline & Total-to-HDL cholesterol ratio (unit) & 0.051 & 7.2 & 0.367 \\
\hline & BMI $\left(\mathrm{kg} / \mathrm{m}^{2}\right)$ & 0.041 & 35 & 1.435 \\
\hline & Estimated common CIMT Z-score & & & 1.322 \\
\hline & Percentile $^{\mathrm{f}}$ & & & $91^{\text {st }}$ \\
\hline \multirow[t]{9}{*}{ Woman } & Intercept $=-1.507^{\mathrm{c}}$ & & & \\
\hline & Age (years) ${ }^{d}$ & -0.027 & 50 & $-0.135^{f}$ \\
\hline & SBP $(10 \mathrm{~mm} \mathrm{Hg})$ & 0.097 & 130 & $1.261^{f}$ \\
\hline & Smoking (yes) & 0.111 & No $(0)$ & 0 \\
\hline & Diabetes (yes) & 0.189 & No $(0)$ & 0 \\
\hline & Total-to-HDL cholesterol ratio (unit) & 0.043 & 3.9 & 0.168 \\
\hline & BMI $\left(\mathrm{kg} / \mathrm{m}^{2}\right)$ & 0.017 & 24 & 0.408 \\
\hline & Estimated common CIMT Z-score & & & 0.195 \\
\hline & Percentile & & & $58^{t h}$ \\
\hline
\end{tabular}

${ }^{a}$ Multiple linear regression coefficients for each risk factor (retrieved from Table 4); ${ }^{b}$ The intercepts provided here are those associated with the regression model in Table 4; ' Hypothetical risk factor values for a male and female subject; ${ }^{d}$ The coefficient for age reflects the residual influence of age on the common CIMT Z-score that was not already accounted for by equations 1 to 4 in this sub-population; ${ }^{e}$ Estimated common CIMT Z-score was calculated as the sum of the intercept and the individual coefficient by risk factor products and compares individual's value (in SD) to mean values among healthy subjects from the same age and sex; ${ }^{f}$ Percentiles for each calculated Z-score can be retrieved by any Standard Normal Distribution (Z) Table; ${ }^{g}$ Note that these coefficients are expressed per 10 years and $10 \mathrm{~mm} \mathrm{Hg}$, respectively, and therefore the products were computed as 50/10*0.020 (man) or 50/10*-0.027 (woman) and $160 / 10 * 0.111$ (man) or 130/10*0.097 (woman). 


\section{ADDITIONAL ANALYSES}

We have also investigated whether the associations between CV-RFs and common CIMT Zscores were modified by age, by adding interaction terms between the CV-RFs and age to our models. We found only significant interaction between age and SBP in the subpopulation without prior CVD and treatment only, and both in men $\left(p_{\text {interaction }}<0.001\right)$ and women $\left(p_{\text {interaction }}=0.033\right.$ ). This suggests that only in the absence of treatment and/or prior CVD the impact of SBP on the common CIMT percentiles may be stronger among older than younger individuals (Table A5.4 and Figure A5.2 - Appendix).

\section{DISCUSSION}

In the present study we estimated age- and sex-specific percentiles (RIs) of common CIMT obtained with echotracking in individuals aged 15-85 years, based on a large population obtained by combining data at the individual level from 24 research centres worldwide. We additionally assessed the relation of CV-RFs with these common CIMT percentiles to enable comparison of common CIMT values obtained across (patient) groups with different cardiovascular risk profiles with those from a healthy population, helping interpretation of such measures obtained both in research and clinical settings.

Common CIMT has been widely used as a surrogate marker for CVD risk in clinical trials. CIMT measurements have also been proposed for screening and fine tuning of individuals' risk prediction ${ }^{8,41-45}$ as ascertained by current risk algorithms such as the Framingham ${ }^{29}$ and SCORE. ${ }^{46}$ Although in some studies CIMT has not improved the discriminative value of these risk scores (i.e. the AUC or the C-statistic) ${ }^{42-44}$ or did not lead to significant reclassification ${ }^{42,43}$ when used additionally to traditional CV-RFs [i.e. age, sex, $\mathrm{BP}$, smoking, diabetes and cholesterol levels], some studies have indeed shown that CIMT may have additional value in terms of significant reclassification, particularly among those at intermediate risk. ${ }^{41,45}$ These observations were confined to men ${ }^{45}$ or women ${ }^{41}$ only, however. Further and ongoing (IMPROVE, USE-IMT) studies (also in populations with different risk profiles) will determine the added value, if any, of common CIMT (echotracking) measurements in individuals' risk stratification. For that purpose, RIs as presented herein may be helpful. In the present study we chose to include echotracking data only to enable optimal comparison across current and future studies, since, at present, echotracking is probably the most accurate method to assess carotid properties. $^{17-19}$

We found that, in the healthy sub-population, common CIMT was higher in men than in women but increased with aging to a similar extent in men (5.2 $\mu \mathrm{m} / \mathrm{year})$ and 
women $(5.0 \mu \mathrm{m} /$ year). These rates are comparable with those previously reported in healthy individuals. ${ }^{15}$ Given the cross-sectional design of these studies, these data need to be interpreted with caution, because these may misestimate the longitudinal rates of change in common CIMT within individuals. Indeed, considerably higher rates of change in common CIMT have been reported in individuals from the longitudinal ARIC study (8.6 and $9.1 \mu \mathrm{m} /$ year in men and women, respectively, age $45-64$ years at baseline) ${ }^{10}$ and in patients from control groups enrolled in lipid-lowering trials $(14.7 \mu \mathrm{m} / \mathrm{year}$, age $\geq 45$ years), ${ }^{5}$ but these common CIMT data were not obtained with echotracking techniques as included in the present study. Although large-scale data on common CIMT progression rates among individuals who are and remain healthy (i.e. free of CV-RFs and CVD) are currently lacking, the rates we reported for the healthy sub-population were quite similar to those described in two well-characterized longitudinal cohorts of young and healthy adults, despite the different methods of CIMT assessment used in these studies. ${ }^{47,48}$

In the sub-population without prior CVD and treatment, we found that SBP, smoking, diabetes, total-to-HDL cholesterol ratio and BMI were significant determinants of higher common CIMT in both men and women, an observation that is largely in line with previous studies. $^{9-11,15}$ The confidence intervals around the association estimates for diabetes and smoking were wider than for the other CV-RFs considered. Factors such as the dichotomous scale (vs. continuous in other CV-RFs), but also the low prevalence of (untreated) diabetes in this study population ( 4 and $3 \%$ in men and women, respectively), differences in the definition of diabetes across centres (e.g. based on self-reports vs. OGTT tests), and self-reported data on smoking may have influenced the precision of these estimates. The observed interaction between age and SBP suggests that, only in the absence of treatment and/or prior CVD, the impact of SBP on the common CIMT percentiles may be stronger among older than younger individuals. This may reflect that age represents the time of exposition to CV-RFs and thereby truly represents the natural history of CV-RFs, explaining the lack of such interaction in the presence of treatment or prior CVD. A note of caution around the meaningfulness of this observation is needed, however. Given the large number of interactions tested (36 in total), we cannot discard the possibility that the interactions between age and SBP may have resulted from the play of chance.

CV-RFs such as diabetes and total-to-HDL cholesterol (in the sub-population with prior CVD and/or BP-, lipid- and/or glucose-lowering medication) and also smoking (in the sub-population with CVD), were not associated with common CIMT Z-scores. These results may illustrate the phenomenon of index event bias, ${ }^{49}$ resulting in apparently differential risk factors for disease after an event (the index event) has occurred, and possible postevent lifestyle and/or treatment changes that may mask the 'effects' of the traditional CVRFs. 
The strength of the associations of some CV-RFs with common CIMT Z-scores differed between sexes such that, BMI (in all subpopulations) and smoking (except in the subpopulation without prior CVD but on treatment) were more strongly associated with increases in common CIMT percentiles (as established in the healthy population) in men than in women. Previous studies have also reported stronger associations of smoking ${ }^{10,15}$ with CIMT in men than in women. However, our findings seem not to link directly to the sex-specific associations between CV-RFs and incident CVD as reported in (recent metaanalyses of) prospective cohort studies. ${ }^{50-55}$ For instance, smoking and diabetes were stronger RFs for incident coronary heart disease in women than in men, ${ }^{50-52}$ whereas no such significant sex interactions have been reported in the associations of BMI and SBP with incident myocardial infarction and stroke. ${ }^{53-55}$ The underlying pathophysiological mechanisms explaining sex differences in the impact of CV-RFs on CIMT and/or CVD remain largely unknown and the current results may therefore only be used for hypothesis-generating purposes.

The influence of carotid diameter, an important determinant of (common) CIMT in the context of arterial remodelling, ${ }^{56}$ was not taken into account in the current study. Studies investigating the influence of carotid diameter on the associations between common CIMT and incident CVD have shown that either adjustment for diameter or calculation of a wall cross-sectional area ('arterial mass') yielded similar associations with myocardial infarction ${ }^{56,57}$ and stroke $^{56}$ to those obtained using common CIMT values alone. Including diameter may thus be necessary in aetiological studies investigating carotid artery remodelling (possibly maladaptive) processes, which are also associated with poorer cardiovascular outcome, rather than in those investigating atherosclerosis in general.

This study has some limitations. First, we standardised differences in techniques between studies/centres also by taking into account other factors potentially influencing common CIMT and differing between centres. However, the calibration may still have been sub-optimal in the case we have missed differences (e.g. in characteristics and/or measurement techniques) between studies/centres, which might have influenced the results. Nevertheless, these differences also increase the heterogeneity of the study population and thereby the external validity of the results. Second, several studies have suggested that ethnicity ${ }^{8,14,58}$ and latitude ${ }^{9}$ may influence common CIMT values. We did not examine the influence of these factors in the present study because we lacked sufficient variability to do so. Specifically, the bulk of the data in the current study originated from a 'Caucasian' European population. The potential influence of ethnicity and/or latitude on common CIMT values, however, may, to a great extent, have been captured by differences in CV-RFs between individuals, which we did examine. Third, in the present study we chose to include common CIMT data obtained using pure echotracking ( $88 \%$ of the data) or related techniques (12\%) only, thus the present results 
might not fully apply to common CIMT data obtained by manual or other automated (imaging) edge-detection systems if not scaled against echotracking techniques. However, age- and sex-specific percentiles for common CIMT presented herein are comparable to those from smaller studies reported previously with CIMT data obtained using other automated edge-detection systems. ${ }^{15}$ Last, the RIs provided do not necessarily translate to increased CVD risk as we did not link these to hard cardiovascular outcome. However, the cut-off values for increasing percentiles indicate deviation from the healthy population means, which was amplified in the presence of CV-RFs, and thus most likely indicate increased risk. Still, the extent to which these cut-offs should guide initiation of therapy needs to be further tested.

In conclusion, we estimated age- and sex-specific percentiles of common CIMT in a healthy population and assessed the relation of CV-RFs with CIMT Z-scores, which enables comparison of CIMT values for (patient) groups with different cardiovascular risk profiles, helping interpretation of such measures obtained both in research and clinical settings. 


\section{REFERENCES}

1. Pignoli P, Tremoli E, Poli A, Oreste P, Paoletti R. Intimal plus medial thickness of the arterial wall: A direct measurement with ultrasound imaging. Circulation. 1986;74:1399-1406

2. Lorenz MW, Markus HS, Bots ML, Rosvall M, Sitzer M. Prediction of clinical cardiovascular events with carotid intima-media thickness: A systematic review and meta-analysis. Circulation. 2007;115:459-467

3. O'Leary DH, Bots ML. Imaging of atherosclerosis: Carotid intima-media thickness. Eur Heart J. 2010;31:16821689

4. Peters SA, Grobbee DE, Bots ML. Carotid intima-media thickness: A suitable alternative for cardiovascular risk as outcome? Eur J Cardiovasc Prev Rehabil. 2011;18:167-174

5. Bots ML, Evans GW, Riley WA, Grobbee DE. Carotid intima-media thickness measurements in intervention studies: Design options, progression rates, and sample size considerations: A point of view. Stroke. 2003;34:2985-2994

6. Touboul PJ, Hennerici MG, Meairs S, Adams H, Amarenco P, Bornstein N, et al. Mannheim carotid intimamedia thickness consensus (2004-2006). An update on behalf of the advisory board of the 3rd and 4th watching the risk symposium, 13th and 15th european stroke conferences, mannheim, germany, 2004, and brussels, belgium, 2006. Cerebrovasc Dis. 2007;23:75-80

7. Urbina EM, Williams RV, Alpert BS, Collins RT, Daniels SR, Hayman L, et al. Noninvasive assessment of subclinical atherosclerosis in children and adolescents: Recommendations for standard assessment for clinical research: A scientific statement from the american heart association. Hypertension. 2009;54:919950

8. Stein JH, Korcarz CE, Hurst RT, Lonn E, Kendall CB, Mohler ER, et al. Use of carotid ultrasound to identify subclinical vascular disease and evaluate cardiovascular disease risk: A consensus statement from the american society of echocardiography carotid intima-media thickness task force. Endorsed by the society for vascular medicine. J Am Soc Echocardiogr. 2008;21:93-111; quiz 189-190

9. Baldassarre D, Nyyssonen K, Rauramaa R, de Faire U, Hamsten A, Smit AJ, et al. Cross-sectional analysis of baseline data to identify the major determinants of carotid intima-media thickness in a european population: The improve study. Eur Heart J. 2010;31:614-622

10. Chambless LE, Folsom AR, Davis V, Sharrett R, Heiss G, Sorlie $P$, et al. Risk factors for progression of common carotid atherosclerosis: The atherosclerosis risk in communities study, 1987-1998. Am J Epidemiol. 2002;155:38-47

11. Held C, Hjemdahl P, Eriksson SV, Bjorkander I, Forslund L, Rehnqvist N. Prognostic implications of intimamedia thickness and plaques in the carotid and femoral arteries in patients with stable angina pectoris. Eur Heart J. 2001;22:62-72

12. van der Meer IM, Iglesias del Sol A, Hak AE, Bots ML, Hofman A, Witteman JC. Risk factors for progression of atherosclerosis measured at multiple sites in the arterial tree: The rotterdam study. Stroke. 2003;34:23742379

13. Ciccone MM, Balbarini A, Teresa Porcelli M, Santoro D, Cortese F, Scicchitano P, et al. Carotid artery intimamedia thickness: Normal and percentile values in the italian population (camp study). Eur J Cardiovasc Prev Rehabil. 2011;18:650-655

14. Howard G, Sharrett AR, Heiss G, Evans GW, Chambless LE, Riley WA, et al. Carotid artery intimal-medial thickness distribution in general populations as evaluated by b-mode ultrasound. Aric investigators. Stroke. 1993;24:1297-1304

15. Sinning C, Wild PS, Echevarria FM, Wilde S, Schnabel R, Lubos E, et al. Sex differences in early carotid atherosclerosis (from the community-based gutenberg-heart study). Am J Cardiol. 2011;107:1841-1847

16. Youn YJ, Lee NS, Kim JY, Lee JW, Sung JK, Ahn SG, et al. Normative values and correlates of mean common carotid intima-media thickness in the korean rural middle-aged population: The atherosclerosis risk of rural areas in korea general population (arirang) study. J Korean Med Sci. 2011;26:365-371

17. Girerd X, Mourad JJ, Acar C, Heudes D, Chiche S, Bruneval P, et al. Noninvasive measurement of mediumsized artery intima-media thickness in humans: In vitro validation. J Vasc Res. 1994;31:114-120

18. Hoeks AP, Willekes C, Boutouyrie P, Brands PJ, Willigers JM, Reneman RS. Automated detection of local artery wall thickness based on m-line signal processing. Ultrasound Med Biol. 1997;23:1017-1023 
19. Girerd X, Boutouyrie P, Pannier B, Mourad JJ, Safar M, Laurent S. Noninvasive ultrasound methods for the measurement of arterial wall thickness In: Touboul PJ, ed. Intima-media thickness and atherosclerosis: Predicting the risk? New York: Parthenon Publishing; 1996:45-58.

20. Laurent S, Caviezel B, Beck L, Girerd X, Billaud E, Boutouyrie P, et al. Carotid artery distensibility and distending pressure in hypertensive humans. Hypertension. 1994;23:878-883

21. Boutouyrie P, Bussy C, Lacolley P, Girerd X, Laloux B, Laurent S. Association between local pulse pressure, mean blood pressure, and large-artery remodeling. Circulation. 1999;100:1387-1393

22. Ferreira I, Twisk JW, van Mechelen W, Kemper HC, Seidell JC, Stehouwer CD. Current and adolescent body fatness and fat distribution: Relationships with carotid intima-media thickness and large artery stiffness at the age of 36 years. J Hypertens. 2004;22:145-155

23. Ferreira I, van de Laar RJ, Prins MH, Twisk JW, Stehouwer CD. Carotid stiffness in young adults: A life-course analysis of its early determinants: The amsterdam growth and health longitudinal study. Hypertension. 2011

24. Henry RM, Kostense PJ, Dekker JM, Nijpels G, Heine RJ, Kamp O, et al. Carotid arterial remodeling: A maladaptive phenomenon in type 2 diabetes but not in impaired glucose metabolism: The hoorn study. Stroke. 2004;35:671-676

25. Henry RM, Kostense PJ, Spijkerman AM, Dekker JM, Nijpels G, Heine RJ, et al. Arterial stiffness increases with deteriorating glucose tolerance status: The hoorn study. Circulation. 2003;107:2089-2095

26. Geerts CC, Evelein AM, Bots ML, van der Ent CK, Grobbee DE, Uiterwaal CS. Body fat distribution and early arterial changes in healthy 5-year-old children. Ann Med. 2011

27. Mancia G, De Backer G, Dominiczak A, Cifkova R, Fagard R, Germano G, et al. 2007 guidelines for the management of arterial hypertension: The task force for the management of arterial hypertension of the european society of hypertension (esh) and of the european society of cardiology (esc). Eur Heart J. 2007;28:1462-1536

28. WHO. Definition, diagnosis, and classification of diabetes mellitus and its complications. Report of a who consultation. Part 1: Diagnosis and classification of diabetes mellitus. Geneva, Switzerland: World Health Organization; 1999.

29. Wilson PW, D'Agostino RB, Levy D, Belanger AM, Silbershatz H, Kannel WB. Prediction of coronary heart disease using risk factor categories. Circulation. 1998;97:1837-1847

30. WHO. Obesity: Preventing and managing the global epidemic. Geneva, Switzerland: World Health Organization; 2000.

31. Espeland MA, Tang R, Terry JG, Davis DH, Mercuri M, Crouse JR, 3rd. Associations of risk factors with segment-specific intimal-medial thickness of the extracranial carotid artery. Stroke. 1999;30:1047-1055

32. Brands PJ, Hoeks AP, Willigers J, Willekes C, Reneman RS. An integrated system for the non-invasive assessment of vessel wall and hemodynamic properties of large arteries by means of ultrasound. Eur $J$ Ultrasound. 1999;9:257-266

33. Segers P, Rabben SI, De Backer J, De Sutter J, Gillebert TC, Van Bortel L, et al. Functional analysis of the common carotid artery: Relative distension differences over the vessel wall measured in vivo. $J$ Hypertens. 2004;22:973-981

34. Zureik M, Temmar M, Adamopoulos C, Bureau JM, Courbon D, Thomas F, et al. Carotid plaques, but not common carotid intima-media thickness, are independently associated with aortic stiffness. J Hypertens. 2002;20:85-93

35. Bianchini E, Bozec E, Gemignani V, Faita F, Giannarelli C, Ghiadoni L, et al. Assessment of carotid stiffness and intima-media thickness from ultrasound data: Comparison between two methods. J Ultrasound Med. 2010;29:1169-1175

36. Sterne JA, White IR, Carlin JB, Spratt M, Royston P, Kenward MG, et al. Multiple imputation for missing data in epidemiological and clinical research: Potential and pitfalls. BMJ. 2009;338:b2393

37. Janssen KJ, Donders AR, Harrell FE, Jr., Vergouwe $Y$, Chen $Q$, Grobbee DE, et al. Missing covariate data in medical research: To impute is better than to ignore. J Clin Epidemiol. 2010;63:721-727

38. Wright EM, Royston P. Calculating reference intervals for laboratory measurements. Stat Methods Med Res. 1999;8:93-112

39. Royston P, Wright E. A method for estimating age-specific reference intervals ('normal ranges') based on fractional polynomials and exponential transformation. J R Statist Soc A. 1998;161:79-101

40. Wright E, Royston P. Age-specific reference intervals for normally distributed data. Stata Technical Bulletin. 1997;38:4-9 
41. Elias-Smale SE, Kavousi M, Verwoert GC, Koller MT, Steyerberg EW, Mattace-Raso FU, et al. Common carotid intima-media thickness in cardiovascular risk stratification of older people: The rotterdam study. Eur J Cardiovasc Prev Rehabil. 2011

42. Polak JF, Pencina MJ, Pencina KM, O'Donnell CJ, Wolf PA, D'Agostino RB, Sr. Carotid-wall intima-media thickness and cardiovascular events. N Engl J Med. 2011;365:213-221

43. Lorenz MW, Schaefer C, Steinmetz H, Sitzer M. Is carotid intima media thickness useful for individual prediction of cardiovascular risk? Ten-year results from the carotid atherosclerosis progression study (caps). Eur Heart J. 2010;31:2041-2048

44. del Sol Al, Moons KG, Hollander M, Hofman A, Koudstaal PJ, Grobbee DE, et al. Is carotid intima-media thickness useful in cardiovascular disease risk assessment? The rotterdam study. Stroke. 2001;32:1532-1538

45. Nambi V, Chambless L, Folsom AR, He M, Hu Y, Mosley T, et al. Carotid intima-media thickness and presence or absence of plaque improves prediction of coronary heart disease risk: The aric (atherosclerosis risk in communities) study. J Am Coll Cardiol. 2010;55:1600-1607

46. Conroy RM, Pyorala K, Fitzgerald AP, Sans S, Menotti A, De Backer G, et al. Estimation of ten-year risk of fatal cardiovascular disease in europe: The score project. Eur Heart J. 2003;24:987-1003

47. Schouten F, Twisk JW, de Boer MR, Stehouwer CD, Serne EH, Smulders YM, et al. Increases in central fat mass and decreases in peripheral fat mass are associated with accelerated arterial stiffening in healthy adults: The amsterdam growth and health longitudinal study. Am J Clin Nutr. 2011;94:40-48

48. Koskinen J, Magnussen CG, Taittonen L, Rasanen L, Mikkila V, Laitinen T, et al. Arterial structure and function after recovery from the metabolic syndrome: The cardiovascular risk in young finns study. Circulation. 2010;121:392-400

49. Dahabreh IJ, Kent DM. Index event bias as an explanation for the paradoxes of recurrence risk research. JAMA. 2011;305:822-823

50. The Emerging Risk Factors Collaboration, Sarwar N, Gao P, Seshasai SR, Gobin R, Kaptoge S, et al. Diabetes mellitus, fasting blood glucose concentration, and risk of vascular disease: A collaborative meta-analysis of 102 prospective studies. Lancet. 2010;375:2215-2222

51. Huxley R, Barzi F, Woodward M. Excess risk of fatal coronary heart disease associated with diabetes in men and women: Meta-analysis of 37 prospective cohort studies. Bmj. 2006;332:73-78

52. Huxley RR, Woodward M. Cigarette smoking as a risk factor for coronary heart disease in women compared with men: A systematic review and meta-analysis of prospective cohort studies. Lancet. 2011;378:12971305

53. Kizer JR, Biggs ML, Ix JH, Mukamal KJ, Zieman SJ, de Boer IH, et al. Measures of adiposity and future risk of ischemic stroke and coronary heart disease in older men and women. Am J Epidemiol. 2011;173:10-25

54. Li C, Engstrom G, Hedblad B, Calling S, Berglund G, Janzon L. Sex differences in the relationships between bmi, whr and incidence of cardiovascular disease: A population-based cohort study. Int J Obes (Lond). 2006;30:1775-1781

55. Vasan RS, Larson MG, Leip EP, Evans JC, O'Donnell CJ, Kannel WB, et al. Impact of high-normal blood pressure on the risk of cardiovascular disease. N Engl J Med. 2001;345:1291-1297

56. Bots ML, Hofman A, Grobbee DE. Increased common carotid intima-media thickness. Adaptive response or a reflection of atherosclerosis? Findings from the rotterdam study. Stroke. 1997;28:2442-2447

57. Bots ML, Grobbee DE, Hofman A, Witteman JC. Common carotid intima-media thickness and risk of acute myocardial infarction: The role of lumen diameter. Stroke. 2005;36:762-767

58. Tzou WS, Douglas PS, Srinivasan SR, Bond MG, Tang R, Li S, et al. Distribution and predictors of carotid intima-media thickness in young adults. Prev Cardiol. 2007;10:181-189 


\section{APPENDIX}

Table A5.1 Author list and participating centres/studies

\begin{tabular}{|c|c|c|}
\hline Centre & Authors & Affiliations \\
\hline $\begin{array}{l}\text { Paris-HEGP } \\
\text { (F) }\end{array}$ & $\begin{array}{l}\text { Pierre Boutouyrie }^{1,2,3,4}, \\
\text { Stéphane Laurent }^{1,2,3,4} \text {, Xavier } \\
\text { Jouven }^{1,2,3} \text {, Jean-Philippe } \\
\text { Empana }^{1,2,3} \text {, Erwan Bozec } \\
\text { Tabassome Simon }^{1,2,3,4}, \text { Bruno }^{\prime} \\
\text { Pannier }\end{array}$ & $\begin{array}{l}{ }^{1} \text { Université Paris Descartes, }{ }^{2} \text { INSERM U970, }{ }^{3} \text { Sorbonne Paris } \\
\text { cité, }{ }^{4} \text { Dept. Pharmacology, Hôpital Europeén Georges } \\
\text { Pompidou, }{ }^{5} \text { Paris VI University, }{ }^{6} \text { Dept. Pharmacology, Saint } \\
\text { Antoine University Hospital, }{ }^{7} \text { Institut Prévention Santé; all } \\
\text { Paris, France }\end{array}$ \\
\hline $\begin{array}{l}\text { Rotterdam } \\
(\mathrm{NL})\end{array}$ & $\begin{array}{l}\text { Francesco Mattace-Raso }{ }^{1,2} \\
\text { Jacqueline Witteman }^{1}\end{array}$ & $\begin{array}{l}{ }^{1} \text { Dept. Epidemiology, }{ }^{2} \text { Dept. Internal Medicine; both Erasmus } \\
\text { University Medical Center Rotterdam, The Netherlands }\end{array}$ \\
\hline Ghent (BE) & $\begin{array}{l}\text { Ernst Rietzschel }^{1} \text {, Sebastian } \\
\text { Vermeersch }^{2,3}, \text { Patrick }^{2,3} \\
\text { Segers }^{2,} \text {, Luc Van Bortel } \\
\text {, Dirk } \\
\text { De Bacquer }{ }^{4}, \text { Caroline Van }^{1} \\
\text { daele }^{1}, \text { Marc De Buyzere }^{1}\end{array}$ & $\begin{array}{l}{ }^{1} \text { Dept. Cardiovascular Disease, Ghent University Hospital, } \\
{ }^{2} \text { IBiTech - bioMMeda, }{ }^{3} \text { Heymans Institute of Pharmacology, } \\
{ }^{4} \text { Dept. Public Health; all Ghent University, Ghent, Belgium }\end{array}$ \\
\hline Utrecht (NL) & $\begin{array}{l}\text { Michiel Bots }{ }^{1}, \text { Yvonne van der }^{1} \\
\text { Schouw }^{1}, \text { Diederick Grobbee }^{1} \text {, } \\
\text { Cuno Uiterwaal }{ }^{1}, \text { Annemieke } \\
\text { Evelein }^{1}, \text { Yolanda van der }^{2} \\
\text { Graaf }^{1}, \text { Frank Visseren }^{2}\end{array}$ & $\begin{array}{l}{ }^{1} \text { Julius Center for Health Sciences and Primary Care, }{ }^{2} \text { Dept. } \\
\text { Vascular Medicine; all University Medical Center Utrecht, } \\
\text { Utrecht, the Netherlands }\end{array}$ \\
\hline Maastricht/ & Coen Stehouwer ${ }^{1}$, Isabel & ${ }^{1}$ Dept. Internal Medicine and School for Cardiovascular \\
\hline $\begin{array}{l}\text { Amsterdam } \\
(\mathrm{NL})\end{array}$ & 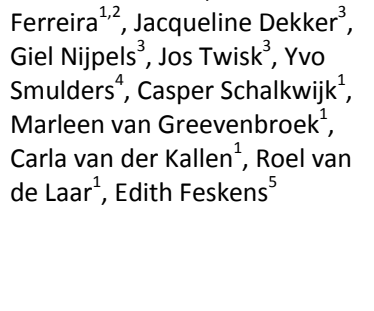 & $\begin{array}{l}\text { Diseases (CARIM), }{ }^{2} \text { Dept. Clinical Epidemiology and Health } \\
\text { Technology Assessment and School for Public Health and } \\
\text { Primary Care (CAPHRI); all Maastricht University Medical } \\
\text { Center, Maastricht, the Netherlands; }{ }^{3} \text { Dept. Epidemiology } \\
\text { and Biostatistics and EMGO Institute for Health and Care } \\
\text { Research, }{ }^{4} \text { Dept. Internal Medicine and Institute of } \\
\text { Cardiovascular Research; all VU University Medical Center, } \\
\text { Amsterdam, the Netherlands; }{ }^{5} \text { Division of Human Nutrition, } \\
\text { Wageningen University, the Netherlands }\end{array}$ \\
\hline Leuven (BE) & $\begin{array}{l}\text { Jan Staessen }{ }^{1,2} \text {, Lutgarde Thijs }{ }^{1} \text {, } \\
\text { Tom Richart }{ }^{1,2}, \text { Tatyana } \\
\text { Kouznetsova }^{1}, \text { Yu Jin }\end{array}$ & $\begin{array}{l}{ }^{1} \text { Studies Coordinating Centre, Division of Hypertension and } \\
\text { Cardiovascular Rehabilitation, Dept. Cardiovascular Diseases, } \\
\text { University of Leuven, Leuven, Belgium, }{ }^{2} \text { Dept. Epidemiology, } \\
\text { Maastricht Uiversity Medical Centre, Maastricht, the } \\
\text { Netherlands, }\end{array}$ \\
\hline Nancy (F) & $\begin{array}{l}\text { Athanase Benetos }{ }^{1,2,3} \text {, Carlos } \\
\text { Labat }^{1,2,3} \text {, Patrick Lacolley }\end{array}$ & $\begin{array}{l}{ }^{1} \text { Nancy University, }{ }^{2} \text { INSERM U961, }{ }^{3} \text { Dept. Geriatrics, Nancy } \\
\text { hospital; all Nancy, France }\end{array}$ \\
\hline $\begin{array}{l}\text { Shanghai } \\
\text { (CN) }\end{array}$ & Jiguang Wang ${ }^{1}$, Yan $\mathrm{Li}^{1}$ & $\begin{array}{l}{ }^{1} \text { Centre for Epidemiological Studies and Clinical Trials, The } \\
\text { Shanghai Institute of Hypertension, Ruijin Hospital, Shanghai } \\
\text { Jiaotong University School of Medicine, Shanghai, China }\end{array}$ \\
\hline $\begin{array}{l}\text { Mannheim } \\
\text { (D) }\end{array}$ & $\begin{array}{l}\text { Joachim Fischer }{ }^{1} \text {, Darcey } \\
\text { Terris }^{1} \text {, Marc Jarczok }{ }^{1} \text {, Maren } \\
\text { Thole }^{1}\end{array}$ & $\begin{array}{l}{ }^{1} \text { Mannheim Institute of Public Health, Social and Preventive } \\
\text { Medicine, Medical Faculty Mannheim, Mannheim, Germany }\end{array}$ \\
\hline Antwerp (BE) & $\begin{array}{l}\text { Hilde Heuten }{ }^{1} \text {, Inge } \\
\text { Goovaerts }{ }^{1} \text {, Guy Ennekens }{ }^{1}\end{array}$ & $\begin{array}{l}{ }^{1} \text { Dept. Cardiology, University Hospital of Antwerp, Edegem, } \\
\text { Belgium }\end{array}$ \\
\hline Vilnius (LT) & $\begin{array}{l}\text { Ligita Ryliskyte }{ }^{1,2}, \text { Aleksandras } \\
\text { Laucevicius }^{1,2}, \text { Kristina } \\
\text { Ryliskiene }^{1,2}\end{array}$ & $\begin{array}{l}{ }^{1} \text { Dept. Cardiovascular Medicine, Vilnius University Hospital } \\
\text { Santariskiu Klinikos, }{ }^{2} \text { Clinic of Cardiac and Vascular Diseases, } \\
\text { Faculty of Medicine, Vilnius University; all Vilnius, Lithuania }\end{array}$ \\
\hline
\end{tabular}


Table A5.1 (continued)

\begin{tabular}{|c|c|c|}
\hline Centre & Authors & Affiliations \\
\hline Pisa (I) & $\begin{array}{l}\text { Elisabetta Bianchini }{ }^{1}, \text { Lorenzo }^{2} \\
\text { Ghiadoni }^{2}, \text { Rosa Maria Bruno }^{2}, \\
\text { Giulia Cartoni }^{2} \text {, Stefano Taddei }\end{array}$ & $\begin{array}{l}{ }^{1} \text { Institute of Clinical Physiology, National Research } \\
\text { Council, }{ }^{2} \text { Dept. Internal Medicine, University of Pisa; all } \\
\text { Pisa, Italy }\end{array}$ \\
\hline São Paulo (BR) & Luiz Bortolotto ${ }^{1}$ & $\begin{array}{l}{ }^{1} \text { Hypertension Unit, Heart Institute, University of São } \\
\text { Paulo Medical School, São Paulo, Brazil }\end{array}$ \\
\hline $\begin{array}{l}\text { Maastricht- } \\
\text { VitaK (NL) }\end{array}$ & $\begin{array}{l}\text { Cees Vermeer }{ }^{1} \text {, Lavienja Braam }{ }^{1} \text {, } \\
\text { Marjo Knapen }{ }^{1}, \text { Nadja } \\
\text { Drummen }\end{array}$ & $\begin{array}{l}{ }^{1} \text { VitaK, Maastricht University Medical Centre, Maastricht, } \\
\text { the Netherlands }\end{array}$ \\
\hline Bern $(\mathrm{CH})$ & $\begin{array}{l}\text { Stefano Rimoldi }{ }^{1,2} \text {, Fabian Stucki, } \\
\text { Damian Hutter }{ }^{1}, \text { Emrush }^{2} \\
\text { Rexhaj }^{2} \text {, Francesco Faita } \\
{ }^{3} \text {, } \\
\text { Claudio Sartori }{ }^{2} \text {, Urs } \\
\text { Scherrer }^{1,2,4}, \text { Yves Allemann }^{1}\end{array}$ & $\begin{array}{l}{ }^{1} \text { Dept. Cardiology, University Hospital of Bern, Bern, } \\
\text { Switzerland, }{ }^{2} \text { Dept. Internal Medicine and Botnar Centre } \\
\text { for Clinical Research, Lausanne, Switzerland, }{ }^{3} \text { Institute of } \\
\text { Clinical Physiology, National Research Council, Pisa, Italy, } \\
{ }^{4} \text { Facultad de Ceincias, Dept. de Biología, Universidad de } \\
\text { Tarapacá, Arica, Chile }\end{array}$ \\
\hline Paris-Foch (F) & Michel Delahousse & ${ }^{1}$ Dept. Nephrology, Hôpital Foch, Suresnes, France \\
\hline Milano/Monza & Cristina Giannattasio ${ }^{1,2}$, & ${ }^{1}$ Dept. Internal Medicine, Milano Bicocca University and \\
\hline ( & $\begin{array}{l}\text { Francesca Cesana }{ }^{1}, \text { Dario Dozio }^{1} \text {, } \\
\text { Alessandro Maloberti }\end{array}$ & $\begin{array}{l}\text { San Gerardo Hospital, Monza, Italy, }{ }^{2} \text { Instituto Auxologico } \\
\text { Italiano, Milan, Italy }\end{array}$ \\
\hline Budapest $(\mathrm{H})$ & $\begin{array}{l}\text { Márk Kollai }{ }^{1}, \text { Alexandra Pintér }{ }^{1} \text {, } \\
\text { Tamás Horváth }^{1}\end{array}$ & $\begin{array}{l}{ }^{1} \text { Institute of Human Physiology and Clinical Experimental } \\
\text { Research, Faculty of Medicine, Semmelweis University, } \\
\text { Budapest, Hungary }\end{array}$ \\
\hline Gdansk (PL) & $\begin{array}{l}\text { Krzysztof Narkiewicz }{ }^{1} \text {, Anna } \\
\text { Szyndler }^{1}, \text { Michał Hoffmann }^{1} \text {, } \\
\text { Robert Nowak }^{1} \text {, Katarzyna } \\
\text { Polonis }\end{array}$ & $\begin{array}{l}{ }^{1} \text { Dept. Hypertension and Diabetology, Medical University } \\
\text { of Gdansk, Gdansk, Poland }\end{array}$ \\
\hline Rouen (F) & $\begin{array}{l}\text { Christian Thuillez } z^{1,2,3} \text {, Robinson } \\
\text { Joannidès }{ }^{1,2,3} \text {, Jérémy Bellien }{ }^{1,2,3}\end{array}$ & $\begin{array}{l}{ }^{1} \text { University of Rouen, }{ }^{2} \text { INSERM U644, }{ }^{3} \text { Dept. } \\
\text { Pharmacology, CHU-Hopitaux de Rouen; all Rouen, } \\
\text { France }\end{array}$ \\
\hline Oslo (N) & Kristin Angel ${ }^{1}$, Dan Atar ${ }^{1}$ & $\begin{array}{l}{ }^{1} \text { Dept. Cardiology, Oslo University Hospital Aker, Oslo, } \\
\text { Norway }\end{array}$ \\
\hline Pilsen (CZ) & Jan Filipovský $^{1}$ & $\begin{array}{l}{ }^{1} \text { Dept. Internal Medicine II, Charles University Medical } \\
\text { Faculty and Teaching Hospital Pilsen, Pilsen, Czech } \\
\text { Republic }\end{array}$ \\
\hline Québec (CDN) & Mohsen Agharazii ${ }^{1}$ & ${ }^{1}$ Dept. Medicine, Université Laval, Québec City, Canada \\
\hline Montreal (CDN) & Marie Briet ${ }^{1}$ & $\begin{array}{l}{ }^{1} \text { Dept. Medicine, Jewish General Hospital, Montréal, } \\
\text { Canada }\end{array}$ \\
\hline
\end{tabular}

$B E$, Belgium; $B R$, Brazil; $C D N$, Canada; $C H$, Switzerland; $C N$, China; $C Z$, Czech Republic; $D$, Germany; $F$, France; $H$, Hungary; I, Italy; $L T$, Lithuania; N, Norway; NL, the Netherlands; PL, Poland. 
Table A5.2 Contributing centres (in order of decreasing number of participating individuals) and respective CIMT measurement techniques used

\begin{tabular}{|c|c|c|c|c|c|c|c|}
\hline $\begin{array}{l}\text { Total } n \text { (healthy } \\
\text { sub-population) }\end{array}$ & Centre & Study name/acronym & Ultrasound device & Carotid artery side & Echotracking system & Width & Anatomical location* \\
\hline \multirow[t]{3}{*}{$6,251(1,341)$} & Paris-HEGP (F) & PPS3 $(n=5,137)$ & Picus (ESAOTE) & right & ART.LAB ${ }^{a}$ & $\geq 10 \mathrm{~mm}$ & Centred at $1 \mathrm{~cm}$ \\
\hline & & HEGP studies ( $n=582)$ & 350 series (ESAOTE) & right & WTS $^{\mathrm{b}}$ & Single M-line & Centred at $2 \mathrm{~cm}$ \\
\hline & & CASHMERE $(n=532)$ & (ESAOTE) & right & WTS & Single M-line & Centred at $2 \mathrm{~cm}$ \\
\hline $5,953(481)$ & Rotterdam (NL) & Rotterdam study & ATL UltraMark IV & left/right & WTS & Single M-line & Centred at $1 \mathrm{~cm}$ \\
\hline $2,524(879)$ & Ghent (BE) & Asklepios & Vivid-7 (GE Vingmed) & left/right & Matlab $^{c}$ & $\geq 10 \mathrm{~mm}$ & $1-2 \mathrm{~cm}$ \\
\hline \multirow[t]{2}{*}{$2,227(7)$} & Utrecht (NL) & SMART $(n=2,079)$ & ATL UltraMark 9 & right & WTS & Single M-line & Centred at $2 \mathrm{~cm}$ \\
\hline & & Whistler Cardio $(n=148)$ & Picus (ESAOTE) & right & ART.LAB & $\geq 10 \mathrm{~mm}$ & Centred at $1 \mathrm{~cm}$ \\
\hline \multirow[t]{3}{*}{$1,633(191)$} & Maastricht/ & Hoorn study ( $n=723)$ & 350 series (ESAOTE) & right & WTS & Single M-line & Centred at $1 \mathrm{~cm}$ \\
\hline & Amsterdam (NL) & AGAHLS $(n=374)$ & 350 series (ESAOTE) & right & WTS & Single M-line & Centred at $1 \mathrm{~cm}$ \\
\hline & & CODAM $(n=536)$ & ATL UltraMark 4+ & left/right & WTS & Single M-line & $1-2 \mathrm{~cm}$ \\
\hline $1,234(308)$ & Leuven (BE) & FLEMENGHO & $\begin{array}{l}\text { ATL UltraMark V }(n=795) \text {; } \\
\text { AU5 (ESAOTE) }(n=439)\end{array}$ & right & WTS & Single M-line & Centred at $2 \mathrm{~cm}$ \\
\hline \multirow[t]{2}{*}{$915(88)$} & Nancy (F) & ERA study $(n=606)$ & Aloka SSD-650 & left/right & $\mathrm{M}^{\prime} \mathrm{ATHS}^{\mathrm{d}}$ & $\geq 10 \mathrm{~mm}$ & Centred at $2 \mathrm{~cm}$ \\
\hline & & ARTEOS study ( $n=309$ ) & (ESAOTE) & right & WTS & Single M-line & Centred at $2 \mathrm{~cm}$ \\
\hline $861(406)$ & Shanghai (CN) & Ningbo Working place & Picus (ESAOTE) & right & ART.LAB & $\geq 10 \mathrm{~mm}$ & $0-1 \mathrm{~cm}$ \\
\hline 738 (99) & Mannheim (D) & Health Check & Picus (ESAOTE) & right & ART.LAB & $\geq 10 \mathrm{~mm}$ & Centred at $1 \mathrm{~cm}$ \\
\hline $491(21)$ & Antwerp (BE) & & (ESAOTE) & right & WTS & Single M-line & Centred at $2 \mathrm{~cm}$ \\
\hline $480(84)$ & Vilnius (LT) & LitHir & Picus (ESAOTE) & left/right & ART.LAB & $\geq 10 \mathrm{~mm}$ & Centred at $1 \mathrm{~cm}$ \\
\hline $401(46)$ & Pisa (I) & CATOD & MyLab 25 (ESAOTE) & left/right & Carotid Studio $^{\mathrm{e}}$ & $\geq 10 \mathrm{~mm}$ & Centred at $1 \mathrm{~cm}$ \\
\hline $303(69)$ & São Paulo (BR) & & Sigma 44 (Kontron) & right & WTS & Single M-line & Centred at $1 \mathrm{~cm}$ \\
\hline 225 (45) & Maastricht-VitaK (NL) & & Picus (ESAOTE) & right & ART.LAB & $\geq 10 \mathrm{~mm}$ & Centred at $2 \mathrm{~cm}$ \\
\hline 208 (59) & Bern $(\mathrm{CH})$ & & MyLab 30 Gold (ESAOTE) & left/right & ART.LAB & $\geq 10 \mathrm{~mm}$ & Centred at $1 \mathrm{~cm}$ \\
\hline $156(2)$ & Paris-Foch (F) & & Picus (ESAOTE) & right & ART.LAB & $\geq 10 \mathrm{~mm}$ & Centred at $1 \mathrm{~cm}$ \\
\hline $153(11)$ & Milano/Monza (I) & & Picus (ESAOTE) & right & ART.LAB & $\geq 10 \mathrm{~mm}$ & Centred at $2 \mathrm{~cm}$ \\
\hline $100(66)$ & Budapest (H) & & L10-5 Picus Pro (ESAOTE) & left & ART.LAB & $\geq 10 \mathrm{~mm}$ & Centred at $1 \mathrm{~cm}$ \\
\hline $90(-)$ & Gdansk (PL) & CareNorth & Picus (ESAOTE) & right & ART.LAB & $\geq 10 \mathrm{~mm}$ & Centred at $1 \mathrm{~cm}$ \\
\hline $65(14)$ & Rouen (F) & & Picus (ESAOTE) & left/right & ART.LAB & $\geq 10 \mathrm{~mm}$ & Centred at $1 \mathrm{~cm}$ \\
\hline $55(15)$ & Oslo (N) & HR study & Picus (ESAOTE) & left/right & ART.LAB & $\geq 10 \mathrm{~mm}$ & Centred at $1 \mathrm{~cm}$ \\
\hline $47(2)$ & Pilsen (CZ) & SAS study & Picus (ESAOTE) & right & ART.LAB & $\geq 10 \mathrm{~mm}$ & Centred at $1 \mathrm{~cm}$ \\
\hline $35(-)$ & Québec (CDN) & & L10-5 Picus Pro (ESAOTE) & right & ART.LAB & $\geq 10 \mathrm{~mm}$ & Centred at $1 \mathrm{~cm}$ \\
\hline $21(-)$ & Montreal (CDN) & & Picus (ESAOTE) & right & ART.LAB & $\geq 10 \mathrm{~mm}$ & Centred at $1 \mathrm{~cm}$ \\
\hline
\end{tabular}

${ }^{*}$ Anatomical location of the measurement is expressed as distance (in $\mathrm{cm}$ ) proximal to the carotid bifurcation; ${ }^{\mathrm{a}} \mathrm{ART}$.LAB echotracking system (ESAOTE, Maastricht, the Netherlands);

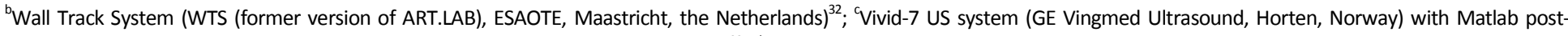
processing (Institute of Biomedical Technology, Ghent University, Ghent, Belgium) ${ }^{33}$; ${ }^{\text {d}}$ Aloka SSD-650 US system (Aloka, Tokyo, Japan) with post-processing in dedicated software (M'ATHS, Metris, France) $)^{34}$; ${ }^{\mathrm{C}}$ Carotid Studio (Institute of Clinical Physiology, National Research Council, Pisa, Italy). ${ }^{35}$ 
Table A5.3 Calibration factors for common CIMT values as obtained with different measurement devices and locations

\begin{tabular}{|c|c|c|c|}
\hline & $\beta$ & $95 \% \mathrm{Cl}$ & $P$-value \\
\hline \multicolumn{4}{|c|}{ Echotracking system $\left(\right.$ reference $=A R T . L A B^{a}$ ) } \\
\hline Wall Track System ${ }^{\mathrm{b}}$ & 127 & $122 ; 131$ & $<0.001$ \\
\hline Vivid-7 + MatLab postprocessing ${ }^{\mathrm{C}}$ & 49 & $36 ; 62$ & $<0.001$ \\
\hline Aloka + M'ATHS postprocessing ${ }^{d}$ & 147 & $135 ; 159$ & $<0.001$ \\
\hline Carotid Studio $^{\mathrm{e}}$ & 80 & $66 ; 93$ & $<0.001$ \\
\hline \multicolumn{4}{|c|}{ Anatomical location (reference $=$ centred at $1 \mathrm{~cm}^{*}$ ) } \\
\hline $0-1 \mathrm{~cm}^{*}$ & 27 & $17 ; 36$ & $<0.001$ \\
\hline $1-2 \mathrm{~cm} *$ & -27 & $-38 ;-15$ & $<0.001$ \\
\hline Centred at $2 \mathrm{~cm}^{*}$ & -72 & $-77 ;-67$ & $<0.001$ \\
\hline
\end{tabular}

The regression coefficient $\beta$ represents the increase in common CIMT (in $\mu \mathrm{m}$ ) when using each of the echotracking systems and anatomical locations listed vs. the reference categories (i.e. ART.LAB and centred at $1 \mathrm{~cm}$ proximal to the carotid bifurcation) at mean levels of age, sex, SBP, smoking, diabetes, total-to-HDL cholesterol ratio, BMI, history of CVD, and use of BP- and/or lipid-lowering medication in the total reference population $(n=24,871)$.

*Anatomical location is expressed as distance (in $\mathrm{cm}$ ) proximal to the carotid bifurcation.

On the basis of this equation, to rescale common CIMT values obtained by for instance Wall Track System (WTS) to values of ART.LAB (i.e. to the values presented in the paper), the original WTS values need to be subtracted by $127 \mu \mathrm{m}$. Likewise, if values were obtained at 0-1 cm, then to rescale these to values of measurements centred at $1 \mathrm{~cm}$ (i.e. to the values presented in the paper), the original $0-1 \mathrm{~cm}$ values need to be subtracted by $27 \mu \mathrm{m}$. 

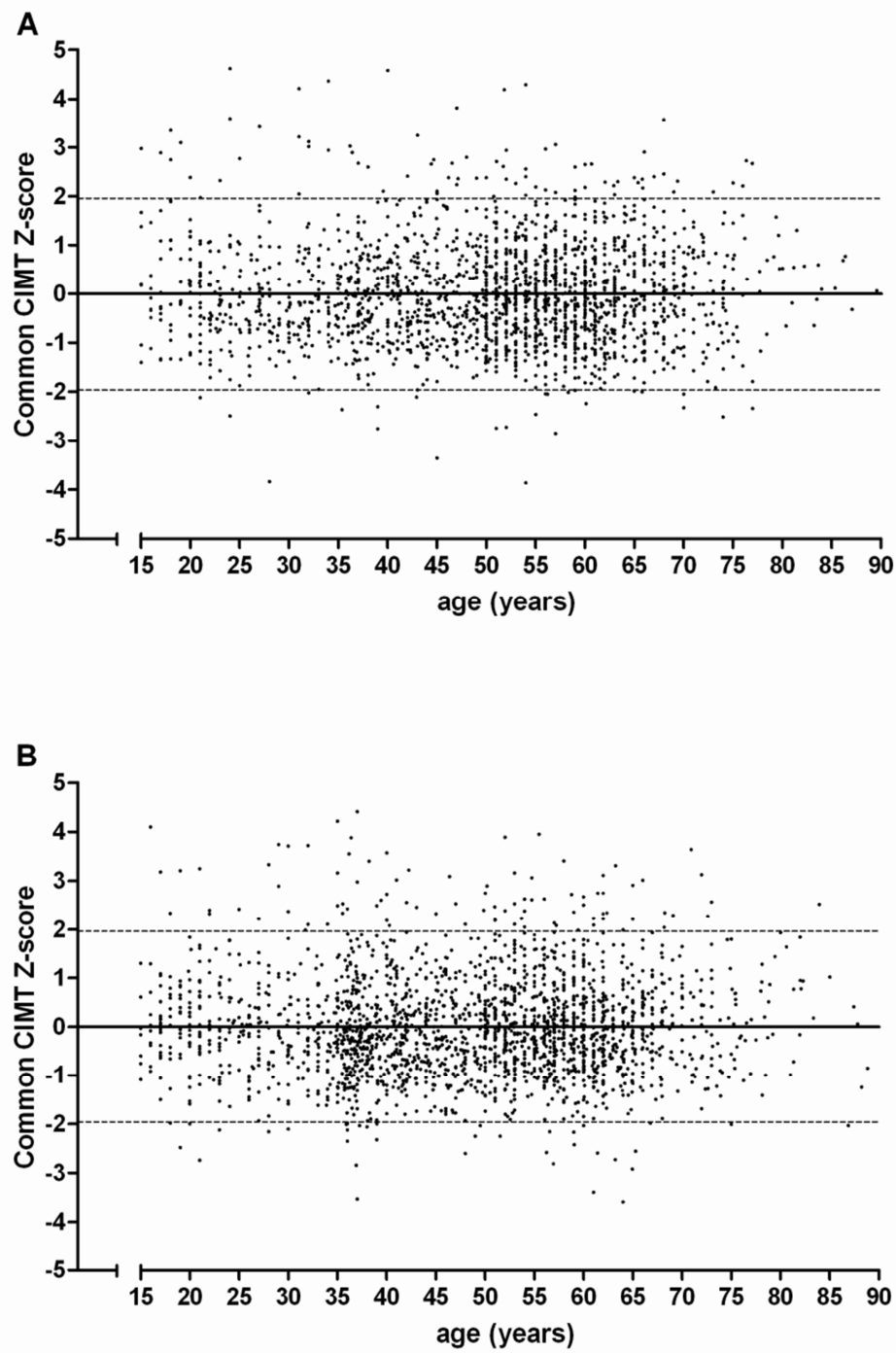

Figure A5.1 Scatter plot of common CIMT Z-scores by age, showing the mean (horizontal line) and +/- 1.96 SD (dotted lines), from the fitted model for common CIMT data for men $(\boldsymbol{A})$ and women $(\boldsymbol{B})$ 
Table A5.4 Associations of SBP with common CIMT Z-scores stratified by age in the sub-population without prior CVD and/or treatment

\begin{tabular}{|c|c|c|c|c|c|c|}
\hline \multirow[b]{2}{*}{ Age categories } & \multicolumn{3}{|c|}{ men } & \multicolumn{3}{|c|}{ women } \\
\hline & $\beta$ & $95 \% \mathrm{Cl}$ & $P$-value & $\beta$ & $95 \% \mathrm{Cl}$ & $P$-value \\
\hline$<40$ years & 0.056 & $0.006 ; 0.105$ & 0.029 & 0.080 & $0.023 ; 0.138$ & 0.006 \\
\hline $40-60$ years & 0.102 & $0.076 ; 0.128$ & $<0.001$ & 0.086 & $0.059 ; 0.112$ & $<0.001$ \\
\hline$>60$ years & 0.132 & $0.105 ; 0.158$ & $<0.001$ & 0.100 & $0.078 ; 0.122$ & $<0.001$ \\
\hline
\end{tabular}

The regression coefficient $\beta$ represents the increase in common CIMT (in SD from the healthy population mean among individuals of the same age and sex) per $10 \mathrm{~mm} \mathrm{Hg}$ increase in SBP. $\beta$ s were obtained from multivariable regression models including all risk factors (SBP, smoking, diabetes, total-HDL-cholesterol ratio and BMI) and age.

A

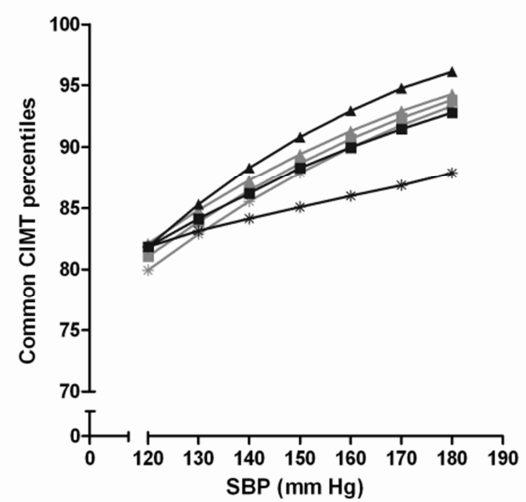

B

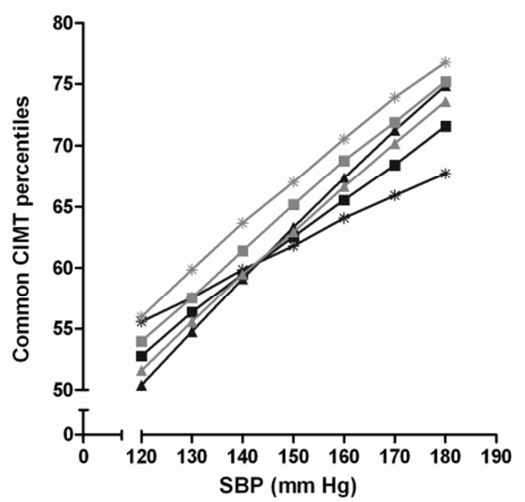

Figure A5.2 The impact of SBP on the estimated common CIMT percentiles with (black) and without (grey) considering interaction between age and SBP for three age examples [i.e. $30(*), 50(\boldsymbol{\square})$ and 70 ( $\boldsymbol{\Delta}$ ) years].

The levels of the other risk factors used for the calculation of the common CIMT percentile [i.e. smoking (yes), diabetes (no), total-HDL-cholesterol (7.2) and BMI $\left(35 \mathrm{~kg} / \mathrm{m}^{2}\right.$ ) - panel A (men), and smoking (no), diabetes (no), total-HDLcholesterol (3.9) and BMI ( $24 \mathrm{~kg} / \mathrm{m}^{2}$ ) - panel B (women) are those of the examples of hypothetical subjects in Table 5. 



\section{CHAPTER $\mathbf{6}$}

\section{POLYMORPHISMS IN S̈LYOXALASE 1 S̈ENE ARE KOT ASSOCIATED WITH VASCULAR COMPLICATIONS: THE HOORM AND CODAM STUDIES}

LIAN EMSELEN, ISABEL FERREIRA, OLAF BROUWERS, ROHALD MA HEMRY,

JACQUELLIEE M DEXKER, SIEEL MIJPELS. ROBERT J HEIME, MARLEEN MJ VAY SRREEYENBROEK, CARLA JH VAN DER KALLEN. ELLEK E BLAAK, EDITH JM FESKENS, HUSO TEN CATE, COEN DA STEHOUWWER, CASPER S SCHALKWIJK

J HYPERTENS. 2009 JUL:27(7):1399-403 


\section{ABSTRACT}

\section{Background}

Methylglyoxal is a major precursor in the formation of advanced glycation endproducts (AGEs), which are known to contribute to vascular complications such as hypertension and arterial stiffness. Methylglyoxal can be detoxified by glyoxalase 1 (GLO1). Because genetic variation in the GLO1 gene may alter the expression and/or the activity of GLO1, we investigated whether single nucleotide polymorphisms (SNPS) in the GLO1 gene are associated with vascular complications.

\section{Methods}

The study entailed cross-sectional data analyses of the CODAM Study and the Hoorn Study, comprising a total of 1,289 subjects, aged $64 \pm 8$ years, of whom $44 \%$ had normal glucose metabolism (NGM), 23\% had impaired glucose metabolism (IGM) and 33\% had type 2 diabetes (T2DM). Nine tag SNPs that cover the common GLO1 gene variation were genotyped. Levels of blood pressure and markers of atherosclerosis, arterial stiffness, renal function and AGEs were compared across genotypes.

\section{Results}

All genotyped SNPs were in Hardy-Weinberg equilibrium. Prevalence of hypertension and markers of atherosclerosis, arterial stiffness, renal function and AGEs did not differ across genotypes of the nine SNPs. In additive models, SNP18 (rs2736654) was associated with pulse pressure $(-1.20 \mathrm{~mm} \mathrm{Hg}(95 \%-\mathrm{Cl}:-2.26 ;-0.14))$ and SNP40 (rs10484854) was associated with systolic blood pressure $(-1.77 \mathrm{~mm} \mathrm{Hg}(-3.40 ;-0.14))$.

\section{Conclusion}

Polymorphisms in the GLO1 gene are not associated with the prevalence of hypertension, markers of atherosclerosis, renal function and AGEs and are weakly associated with pulse pressure and systolic blood pressure (possibly due to chance) in two Dutch cohorts of subjects with NGM, IGM and T2DM. 


\section{INTRODUCTION}

Methylglyoxal, a reactive glycolysis derived dicarbonyl metabolite, is the most reactive precursor in the formation of advanced glycation endproducts (AGEs), ${ }^{1}$ which have been linked to vascular complications. ${ }^{1}$ The deleterious effects of AGEs are attributed to direct modification of extracellular matrix proteins, resulting in collagen cross-links, leading to increased vascular stiffness, as well as via binding to a variety of cell-surface receptors which trigger signal transduction pathways resulting in proinflammatory cellular responses. $^{1,2}$

Additionally, methylglyoxal has been associated with the development of hypertension ${ }^{3,4}$ and it has been shown to increase oxidative stress, ${ }^{3}$ which has been described in relation to hypertension ${ }^{5}$ and cardiovascular disease. ${ }^{6}$

Methylglyoxal can be detoxified by the glyoxalase system, which consists of glyoxalase 1 (GLO1) and glyoxalase 2, into D-lactate. ${ }^{7}$ Convincing experimental evidence demonstrated that GLO1 is the major cellular enzyme that catalyses the metabolism of methylglyoxal and has a critical role in suppressing the formation of AGEs. ${ }^{8,9}$

Because genetic variation in the GLO1 gene may alter the expression and/or the activity of GLO1, we investigated whether the nine single nucleotide polymorphisms (SNPs), which cover the total common variability in the GLO1 gene, were associated with markers of vascular (i.e. hypertension, atherosclerosis, arterial stiffness) and renal complications and levels of AGEs in plasma. These analyses were conducted in a large cohort of Dutch individuals with normal glucose metabolism (NGM), impaired glucose metabolism (IGM) and type 2 diabetes (T2DM).

\section{METHODS}

\section{STUDY POPULATION}

The present study included participants from the follow-up examination of the Hoorn study $^{10}$ and from the CODAM study. ${ }^{11}$

Briefly, the Hoorn Study, which started in 1989, is a population-based cohort study investigating glucose tolerance status and cardiovascular disease risk factors among a sample of the general population in Hoorn, the Netherlands. In 2000-2001, a follow-up examination was conducted in a selected group of 822 subjects consisting of all subjects with T2DM and a random sample of the survival cohort with NGM or IGM. ${ }^{10}$ The CODAM 
Study, which started in 1999-2000, is a cohort study designed to investigate the effects of glucose metabolism, blood lipids, lifestyle, and genetic factors on cardiovascular morbidity and mortality. In total, 574 subjects were enrolled. ${ }^{11}$

Both cohort studies were approved by the local Ethics Committees (VU University Medical Center Amsterdam or Maastricht University Medical Centre, respectively). Written informed consent was obtained from all participants.

Subjects who had missing data for all genotyped SNPs (mainly due to missing DNA samples, $n=107 ; 101$ from Hoorn; 6 from CODAM) were excluded from the analyses. These subjects did not differ from those included with regard to the main outcomes and other covariates investigated (data not shown). The final study population therefore consisted of 1,289 subjects ( 721 from Hoorn, 568 from CODAM), $44 \%$ of whom had NGM, $23 \%$ had IGM and 33\% had T2DM.

\section{SNP SELECTION AND GENOTYPING}

In order to enable for coverage of the total GLO1 gene, SNP selection was based on the GLO1 gene including 3000 base pairs downstream and upstream. Common SNPs (minor allele frequency $(\mathrm{MAF})>5 \%, n=28)$ were selected using HapMap and Haploview. By genotyping nine tag SNPs, these 28 common SNPs were captured at $r^{2}>0.8$ in linkage disequilibrium. The location of these SNPs in the GLO1 gene is shown in Figure 6.1. For genotyping, DNA was extracted from peripheral blood samples according to standard procedures. GLO1 gene SNPs were genotyped using the ABI PRISM 7900HT sequence detection system (Applied Biosystems, Foster City, CA).

\section{BLOOD AND URINE SAMPLES AND BIOCHEMICAL MEASUREMENTS}

Fasting venous blood samples were drawn and serum and plasma were immediately separated after centrifugation and stored at $-80^{\circ} \mathrm{C}$ until assays were performed. ${ }^{10,11}$

Plasma levels of the AGEs $\mathrm{N}^{\varepsilon}$-(carboxymethyl)lysine (CML) and $\mathrm{N}^{\varepsilon}$-(carboxyethyl)lysine (CEL) were measured by high performance liquid chromatography-tandem mass spectrometry in the CODAM study population only.

Renal function was calculated by estimated glomerular filtration rate (eGFR, in $\left.\mathrm{mL} / \mathrm{min} / 1.73 \mathrm{~m}^{2}\right)$ using the MDRD formula $\left(\left[186 *\left((\text { creatinine/88.4 })^{-1.154}\right) *\left(\right.\right.\right.$ age $\left.\left.{ }^{-0.203}\right)\right] *$ 0.742 if female).

Morning urine samples were frozen at $-20 \circ \mathrm{C}$. The albumin concentration was determined by rate nephelometry (Array Protein System, Beckman Inc., Brea, California, 
USA). Microalbuminuria was considered to be present when albumin concentration was 20-200 mg/L and macroalbuminuria when albumin concentration was $>200 \mathrm{mg} / \mathrm{L}$.

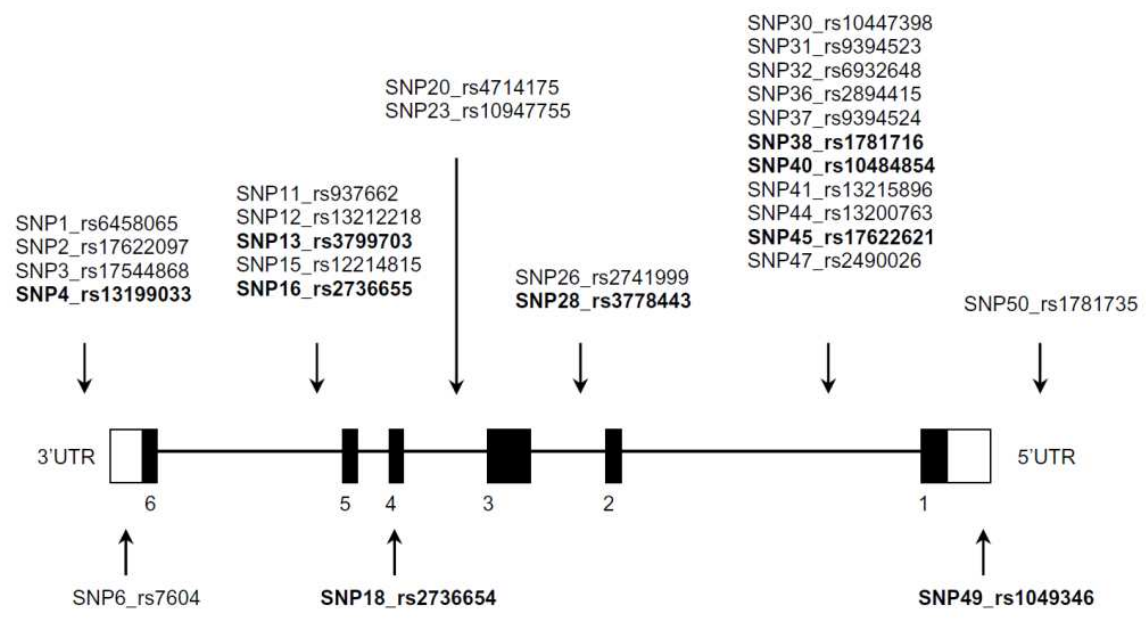

Figure 6.1 Representation of the human glyoxalase 1 gene

Arrows indicate the approximate locations of the common SNPs; the nine SNPs genotyped in this study are printed in bold. Black boxes represent exons and untranslated regions (UTR's) of the first and last exon are indicated as white boxes. SNP1, 2, 3 and 4 are located on the 3' untranslated region; SNP6 on exon 6 (untranslated region); SNP18 on exon 4 (non-synonymous); SNP49 on exon 1 (untranslated region); SNP50 on the 5' untranslated region; and all other SNPs are located on introns.

\section{BLOOD PRESSURE, ATHEROSCLEROSIS AND ARTERIAL STIFFNESS}

Systolic (SBP) and diastolic (DBP) blood pressures were measured using an oscillometric device (Press-Mate, BP-8800; Colin Medical Instruments Corp., San Antonio, Texas, USA in the Hoorn Study) or a high precision aneroid sphygmomanometer (Speidel \& Kelly Maxi Stabil 3, Jungingen, Germany in the CODAM study). Hypertension was defined as SBP $\geq 140$ $\mathrm{mm} \mathrm{Hg}, \mathrm{DBP} \geq 90 \mathrm{~mm} \mathrm{Hg}$ and/or use of antihypertensive medication.

Ankle-brachial index ( $\mathrm{ABI}$ ) and carotid intima-media thickness (CIMT) were used as markers of atherosclerosis. Doppler assisted measures of SBP in the brachial and in the posterior tibial and dorsalis pedis arteries (both sides) were performed with subjects in the supine position. The average SBP was calculated for the posterior tibial and dorsalis pedis arteries in each leg and further used for the $A B I$ calculation by dividing the highest of the ankle pressures by the highest pressure at the brachial arteries. Subjects with $A B \mid>1.5$ 
were excluded from the analyses $(n=5)$. Non-invasive ultrasound imaging techniques and devices (Pie 350 Series; Pie Medical BV, Maastricht, The Netherlands in the Hoorn study and Ultramark 4+, Advance Technology Laboratories, Bothel, Washington, USA in the CODAM study) were used to measure large artery properties in both studies following the same protocols, ${ }^{10}$ which comply with international guidelines for user procedures. Briefly, CIMT was measured in the right common carotid artery and, in addition, but in the Hoorn study only, diameter and distension were obtained from the right common carotid, femoral and brachial arteries. These measures, in combination with local pulse pressures (PP), were used to calculate the following stiffness estimates: the distensibility coefficient (DC, which reflects the elastic properties) and the compliance coefficient (CC, which reflects the buffering capacity) of the three arteries, and the carotid Young's elastic modulus (YEM, an estimate of the intrinsic elastic properties of the vessel wall). ${ }^{10}$

Cardiovascular disease (CVD) was defined as a history of myocardial infarction, stroke or transient ischaemic attack, non-traumatic limb amputation, coronary or peripheral artery bypass, angioplasty, or signs of infarction or ischemia on a 12-lead electrocardiogram (Minnesota codes 1-1, 1-2, 1-3, 4-1, 4-2, 4-3, 5-1, 5-2, 5-3 or 7-1) or $A B \mid<0.9$ in either leg.

\section{STATISTICAL ANALYSES}

Linkage disequilibrium statistics were analyzed by Haploview software. Hardy-Weinberg equilibrium was assessed using a $\chi^{2}$ test. All other statistical analyses were carried out using SPSS, version 15.0 (SPSS Inc., Chicago, IL, USA). The NGM, IGM and T2DM group were compared for genotype frequencies of all SNPs using a $\chi^{2}$ test. Linear and logistic regression analyses were used to compare outcome variables across genotypes of the nine GLO1 SNPS. Dominant- (using major homozygous genotype as a reference), recessive(using major homozygous plus heterozygous genotype as a reference) and additive models (comparison across three genotypes) were used. In addition, whenever associations with individual SNPs were found, haplotypes were constructed using the SNPHAP program (www-gene.cimr.cam.ac.uk/clayton/software).

Each of the stiffness estimates (i.e. DC, CC (both multiplied by -1 ) and YEM) was adjusted for subjects' level of MAP and height prior to further analyses. These adjusted values were then standardized (subjects' value - population mean / SD) and compiled into an overall arterial stiffness sex-specific Z-score, so that a higher score reflects greater stiffness. All analyses were adjusted for age, sex and glucose metabolism status (NGM, IGM or T2DM). 
Table 6.1 Subjects' characteristics for the Hoorn study, CODAM and the total population

\begin{tabular}{|c|c|c|c|}
\hline & Hoorn study & CODAM & Total population \\
\hline$n(\mathrm{~m} / \mathrm{f})$ & $721(361 / 360)$ & $568(347 / 221)$ & $1289(708 / 581)$ \\
\hline Age (years) & $68.8 \pm 7.21$ & $59.1 \pm 6.96$ & $64.5 \pm 8.58$ \\
\hline Body mass index $\left(\mathrm{kg} / \mathrm{m}^{2}\right)$ & $27.7 \pm 4.13$ & $28.6 \pm 4.31$ & $28.1 \pm 4.23$ \\
\hline Waist circumference $(\mathrm{cm})$ & $96.6 \pm 12.1$ & $99.0 \pm 12.5$ & $97.6 \pm 12.3$ \\
\hline NGM/IGM/T2DM (\%) & $36 / 24 / 40$ & $53 / 22 / 25$ & $44 / 23 / 33$ \\
\hline Fasting glucose (mmol/L) & $6.49 \pm 1.55$ & $6.08 \pm 1.48$ & $6.31 \pm 1.54$ \\
\hline Postload glucose (mmol/L) & $7.27 \pm 2.60$ & $7.73 \pm 3.50$ & $7.50 \pm 3.10$ \\
\hline Glycated hemoglobin (\%) & $6.11 \pm 0.77$ & $5.97 \pm 0.82$ & $6.05 \pm 0.80$ \\
\hline Fasting insulin (pmol/L) & $61(43-91)$ & $60(45-89)$ & $60(44-90)$ \\
\hline HOMA-IR & $1.2(0.8-1.8)$ & $1.2(0.8-1.7)$ & $1.2(0.85-1.8)$ \\
\hline Total cholesterol (mmol/L) & $5.67 \pm 1.05$ & $5.22 \pm 0.99$ & $5.47 \pm 1.04$ \\
\hline HDL-cholesterol (mmol/L) & $1.38 \pm 0.40$ & $1.19 \pm 0.35$ & $1.30 \pm 0.39$ \\
\hline LDL-cholesterol (mmol/L) & $3.58 \pm 0.92$ & $3.31 \pm 0.86$ & $3.46 \pm 0.90$ \\
\hline Triglycerides (mmol/L) & $1.58 \pm 0.89$ & $1.65 \pm 1.55$ & $1.61 \pm 1.22$ \\
\hline \multicolumn{4}{|l|}{ Blood pressure } \\
\hline Systolic blood pressure (mm Hg) & $143 \pm 20.3$ & $140 \pm 19.1$ & $142 \pm 19.9$ \\
\hline Diastolic blood pressure (mm Hg) & $83.2 \pm 10.9$ & $81.8 \pm 9.20$ & $82.6 \pm 10.2$ \\
\hline Mean arterial blood pressure $(\mathrm{mm} \mathrm{Hg})$ & $103 \pm 12.4$ & $101 \pm 11.4$ & $102 \pm 12.0$ \\
\hline Pulse pressure $(\mathrm{mm} \mathrm{Hg})$ & $66.4 \pm 15.3$ & $58.4 \pm 14.7$ & $62.7 \pm 15.6$ \\
\hline Hypertension (\%) & 71.5 & 62.1 & 67.4 \\
\hline \multicolumn{4}{|l|}{ Markers of atherosclerosis } \\
\hline Carotid intima-media thickness $(\mathrm{mm})$ & $0.86 \pm 0.17$ & $0.78 \pm 0.16$ & $0.82 \pm 0.17$ \\
\hline Ankle-brachial index & $1.16 \pm 0.14$ & $1.15 \pm 0.13$ & $1.16 \pm 0.14$ \\
\hline Cardiovascular disease (\%) & 48.5 & 27.5 & 39.0 \\
\hline \multicolumn{4}{|l|}{ Markers of arterial stiffness } \\
\hline Carotid DC $\left(10^{-3} \mathrm{kPa}^{-1}\right)$ & $11.5 \pm 4.36$ & - & \\
\hline Carotid CC $\left(\mathrm{mm}^{2} \mathrm{kPa}^{-1}\right)$ & $0.56 \pm 0.23$ & - & \\
\hline Carotid YEM (103kPa) & $0.98 \pm 0.52$ & - & \\
\hline Femoral DC $\left(10^{-3} \mathrm{kPa}^{-1}\right)$ & $4.65 \pm 2.11$ & - & \\
\hline Femoral CC $\left(\mathrm{mm}^{2} \mathrm{kPa}^{-1}\right)$ & $0.36 \pm 0.19$ & - & \\
\hline Brachial DC $\left(10^{-3} \mathrm{kPa}^{-1}\right)$ & $7.52 \pm 4.00$ & - & \\
\hline Brachial CC $\left(\mathrm{mm}^{2} \mathrm{kPa}^{-1}\right)$ & $0.13 \pm 0.07$ & - & \\
\hline \multicolumn{4}{|l|}{ Markers of renal disease } \\
\hline Albuminuria: normo/micro/macro (\%) & $87 / 12 / 1.5$ & $92 / 6.5 / 2.1$ & 89/9.3/1.3 \\
\hline eGFR $\left(\mathrm{mL} / \mathrm{min} / 1.73 \mathrm{~m}^{2}\right)$ & $57.4 \pm 9.85$ & $85.4 \pm 17.0$ & $69.8 \pm 19.4$ \\
\hline \multicolumn{4}{|l|}{ Advanced glycation endproducts } \\
\hline $\mathrm{CML}(\mu \mathrm{mol} / \mathrm{L})$ & - & $1.77 \pm 0.48$ & \\
\hline $\mathrm{CEL}(\mu \mathrm{mol} / \mathrm{L})$ & - & $1.20 \pm 0.40$ & \\
\hline
\end{tabular}

Data are expressed as mean \pm SD, median (inter-quartile range) or percentages. NGM, normal glucose metabolism; IGM, impaired glucose metabolism; T2DM, type 2 diabetes mellitus; HOMA-IR, insulin resistance based on the homeostasis model of assessment; $D C$, distensibility coefficient; $C C$, compliance coefficient; YEM, Young's elastic modulus; eGFR estimated glomerular filtration rate calculated by MDRD formula; CML, plasma $\mathrm{N}^{\varepsilon}$ (carboxymethyl) lysine; $C E L, \mathrm{plasma}^{\varepsilon}$ (carboxyethyl) lysine. 


\section{RESULTS}

Subjects' baseline characteristics for the Hoorn, the CODAM and the total study population are shown in Table 6.1. All genotyped SNPs were in Hardy-Weinberg equilibrium. Genotype frequencies did not differ between the NGM, IGM and the T2DM individuals or between both study populations ( $p$-value $>0.2$ for all; data not shown).

\section{ASSOCIATION BETWEEN GLO1 SNPS AND LEVELS OF BLOOD PRESSURE/HYPERTENSION AND MARKERS OF ATHEROSCLEROSIS AND ARTERIAL STIFFNESS}

Prevalence of hypertension and CVD and levels of CIMT, ABI and arterial stiffness did not differ across genotypes of the nine GLO1 SNPs. However, in an additive model, SNP18 ( $r$ 2736654) was associated with PP, so that PP values decreased by $-1.20 \mathrm{~mm} \mathrm{Hg} \mathrm{(95 \% -Cl:}$ $-2.26 ;-0.14)$ across the $T T, G T$ and GG genotypes. A similar trend was seen for SBP though this did not reach statistical significance $(-1.36 \quad(-2.82 ; 0.12))$. In addition, SNP40 ( $r$ 10484854) was associated with SBP, so that SBP values decreased by $-1.77 \mathrm{~mm} \mathrm{Hg}$ (3.40;-0.14) across the CC, CT and TT genotypes (Table 6.2). Haplotypes compiling SNP18 (rs2736654) and SNP40 (rs10484854) showed an association with SBP (lower in the GT haplotype: $-1.74 \mathrm{~mm} \mathrm{Hg}(95 \%-\mathrm{Cl}:-3.36 ;-0.13))$, but not with PP $(-0.82(-1.99 ; 0.34))$.

\section{ASSOCIATION BETWEEN GLO1 SNPS AND MARKERS OF RENAL FUNCTION AND AGES}

Genotype frequencies did not differ between subjects with normo-, micro- or macroalbuminuria (data not shown). eGFR and plasma levels of CML and CEL did not differ across genotypes of the nine GLO1 SNPs (Table 6.3).

\section{ADDITIONAL ANALYSES}

Similar results were found after further adjustment for study population or when analyses were stratified according to glucose metabolism status or study population. 
Table 6.2 Levels of blood pressure and markers of atherosclerosis and arterial stiffness across genotypes of nine SNPs in the GLO1 gene

\begin{tabular}{|c|c|c|c|c|c|c|c|c|c|}
\hline \multirow[b]{2}{*}{ SNP } & \multirow[b]{2}{*}{ genotype } & \multirow[b]{2}{*}{$n$} & \multicolumn{3}{|c|}{ Blood pressure } & \multicolumn{2}{|c|}{ Atherosclerosis } & \multicolumn{2}{|c|}{ Arterial stiffness $^{a}$} \\
\hline & & & SBP & DBP & PP & CIMT & $A B I$ & $n$ & Z-score \\
\hline \multirow{3}{*}{$\begin{array}{l}\text { SNP4 } \\
\text { (rs13199033) }\end{array}$} & $A A$ & 894 & $141 \pm 0.60$ & $82.7 \pm 0.32$ & $62.5 \pm 0.43$ & $0.82 \pm 0.01$ & $1.16 \pm 0.00$ & 440 & $-0.02 \pm 0.04$ \\
\hline & AT & 216 & $142 \pm 1.24$ & $82.5 \pm 0.66$ & $62.5 \pm 0.89$ & $0.84 \pm 0.01$ & $1.15 \pm 0.01$ & 100 & $0.04 \pm 0.09$ \\
\hline & $\mathrm{TT}$ & 16 & $146 \pm 4.70$ & $83.1 \pm 2.49$ & $66.3 \pm 3.33$ & $0.82 \pm 0.04$ & $1.14 \pm 0.03$ & 8 & $-0.15 \pm 0.33$ \\
\hline \multirow{3}{*}{$\begin{array}{l}\text { SNP13 } \\
\text { (rs3799703) }\end{array}$} & GG & 351 & $141 \pm 0.95$ & $82.7 \pm 0.51$ & $61.5 \pm 0.68$ & $0.83 \pm 0.01$ & $1.16 \pm 0.01$ & 185 & $-0.02 \pm 0.07$ \\
\hline & AG & 535 & $142 \pm 0.77$ & $82.4 \pm 0.42$ & $62.9 \pm 0.55$ & $0.83 \pm 0.01$ & $1.16 \pm 0.01$ & 262 & $-0.02 \pm 0.06$ \\
\hline & $A A$ & 246 & $143 \pm 1.13$ & $83.3 \pm 0.61$ & $63.2 \pm 0.81$ & $0.82 \pm 0.01$ & $1.16 \pm 0.01$ & 117 & $0.05 \pm 0.09$ \\
\hline \multirow{3}{*}{$\begin{array}{l}\text { SNP16 } \\
\text { (rs2736655) }\end{array}$} & GG & 858 & $141 \pm 0.61$ & $82.7 \pm 0.33$ & $62.4 \pm 0.44$ & $0.82 \pm 0.01$ & $1.16 \pm 0.00$ & 424 & $0.01 \pm 0.05$ \\
\hline & AG & 260 & $142 \pm 1.12$ & $81.9 \pm 0.60$ & $63.2 \pm 0.80$ & $0.82 \pm 0.01$ & $1.16 \pm 0.01$ & 124 & $-0.11 \pm 0.08$ \\
\hline & AA & 23 & $141 \pm 3.59$ & $82.7 \pm 1.93$ & $62.5 \pm 2.64$ & $0.82 \pm 0.03$ & $1.17 \pm 0.03$ & 12 & $-0.15 \pm 0.27$ \\
\hline \multirow{3}{*}{$\begin{array}{l}\text { SNP18 }{ }^{b} \\
(\text { rs2736654) }\end{array}$} & $\mathrm{TT}$ & 356 & $143 \pm 0.96$ & $82.7 \pm 0.52$ & $63.8 \pm 0.69$ & $0.82 \pm 0.01$ & $1.16 \pm 0.01$ & 177 & $0.07 \pm 0.07$ \\
\hline & GT & 563 & $142 \pm 0.75$ & $82.5 \pm 0.41$ & $62.3 \pm 0.54$ & $0.82 \pm 0.01$ & $1.15 \pm 0.01$ & 270 & $-0.09 \pm 0.06$ \\
\hline & GG & 227 & $140 \pm 1.17$ & $82.5 \pm 0.63$ & $61.5 \pm 0.85$ & $0.83 \pm 0.01$ & $1.16 \pm 0.01$ & 119 & $0.04 \pm 0.08$ \\
\hline \multirow{3}{*}{$\begin{array}{l}\text { SNP28 } \\
\text { (rs3778443) }\end{array}$} & GG & 1006 & $142 \pm 0.57$ & $82.6 \pm 0.30$ & $62.8 \pm 0.41$ & $0.82 \pm 0.00$ & $1.15 \pm 0.00$ & 510 & $-0.03 \pm 0.04$ \\
\hline & $A G$ & 141 & $143 \pm 1.56$ & $83.7 \pm 0.83$ & $62.6 \pm 1.11$ & $0.84 \pm 0.01$ & $1.16 \pm 0.01$ & 56 & $0.22 \pm 0.12$ \\
\hline & $A A$ & 7 & $138 \pm 7.11$ & $77.8 \pm 3.78$ & $63.2 \pm 5.04$ & $0.83 \pm 0.06$ & $1.22 \pm 0.05$ & 3 & $-0.11 \pm 0.53$ \\
\hline \multirow{3}{*}{$\begin{array}{l}\text { SNP38 } \\
(\text { rs1781716) }\end{array}$} & GG & 963 & $141 \pm 0.58$ & $82.5 \pm 0.31$ & $62.7 \pm 0.42$ & $0.82 \pm 0.01$ & $1.15 \pm 0.00$ & 499 & $-0.04 \pm 0.04$ \\
\hline & CG & 181 & $144 \pm 1.36$ & $83.6 \pm 0.73$ & $63.3 \pm 0.97$ & $0.83 \pm 0.01$ & $1.15 \pm 0.01$ & 75 & $-0.26 \pm 0.11$ \\
\hline & $\mathrm{CC}$ & 8 & $137 \pm 6.64$ & $76.4 \pm 3.57$ & $63.7 \pm 4.7$ & $0.82 \pm 0.06$ & $1.22 \pm 0.05$ & 4 & $-0.25 \pm 0.46$ \\
\hline \multirow{3}{*}{$\begin{array}{l}\text { SNP40 } \\
(\text { rs10484854) }\end{array}$} & $\mathrm{CC}$ & 597 & $142 \pm 0.73$ & $82.9 \pm 0.39$ & $63.2 \pm 0.53$ & $0.82 \pm 0.01$ & $1.15 \pm 0.01$ & 297 & $0.04 \pm 0.05$ \\
\hline & $\mathrm{CT}$ & 469 & $141 \pm 0.82$ & $82.4 \pm 0.44$ & $61.9 \pm 0.59$ & $0.82 \pm 0.01$ & $1.16 \pm 0.01$ & 233 & $-0.07 \pm 0.06$ \\
\hline & $\mathrm{TT}$ & 86 & $138 \pm 1.90$ & $81.2 \pm 1.03$ & $62.1 \pm 1.40$ & $0.84 \pm 0.02$ & $1.15 \pm 0.01$ & 43 & $-0.03 \pm 0.14$ \\
\hline \multirow{3}{*}{$\begin{array}{l}\text { SNP45 } \\
(\mathrm{rs} 17622621)\end{array}$} & GG & 423 & $142 \pm 0.86$ & $83.2 \pm 0.47$ & $63.1 \pm 0.63$ & $0.82 \pm 0.01$ & $1.15 \pm 0.01$ & 208 & $0.02 \pm 0.06$ \\
\hline & $A G$ & 536 & $142 \pm 0.76$ & $82.5 \pm 0.42$ & $62.4 \pm 0.56$ & $0.83 \pm 0.01$ & $1.16 \pm 0.01$ & 265 & $0.01 \pm 0.06$ \\
\hline & $A A$ & 166 & $140 \pm 1.38$ & $81.8 \pm 0.75$ & $62.3 \pm 1.02$ & $0.83 \pm 0.01$ & $1.14 \pm 0.01$ & 78 & $-0.08 \pm 0.10$ \\
\hline \multirow{3}{*}{$\begin{array}{l}\text { SNP49 } \\
\text { (rs1049346) }\end{array}$} & GG & 285 & $142 \pm 1.06$ & $83.3 \pm 0.57$ & $63.3 \pm 0.76$ & $0.82 \pm 0.01$ & $1.15 \pm 0.01$ & 155 & $-0.04 \pm 0.07$ \\
\hline & AG & 588 & $142 \pm 0.73$ & $82.5 \pm 0.40$ & $62.4 \pm 0.53$ & $0.83 \pm 0.01$ & $1.16 \pm 0.01$ & 288 & $0.00 \pm 0.06$ \\
\hline & $A A$ & 277 & $141 \pm 1.10$ & $82.3 \pm 0.59$ & $62.6 \pm 0.79$ & $0.83 \pm 0.01$ & $1.15 \pm 0.01$ & 126 & $0.04 \pm 0.08$ \\
\hline
\end{tabular}

Data are expressed as mean \pm SE. SBP, systolic blood pressure; DBP, diastolic blood pressure; PP, pulse pressure; CIMT, carotid intima-media thickness; $\mathrm{ABI}$, ankle-brachial index; arterial stiffness, seven parameters of arterial stiffness compiled into a z-score adjusted for MAP and height ( ${ }^{a}$ data from the Hoorn Study only). All analyses were adjusted for age, sex and glucose metabolism status. $P$-values for trends across genotypes all $>0.1$, except for ${ }^{b}$ and ${ }^{c}$ in analyses with pulse pressure and systolic blood pressure, respectively. 
Table 6.3 eGFR and plasma levels of AGEs across genotypes of nine SNPs in the GLO1 gene

\begin{tabular}{|c|c|c|c|c|c|c|}
\hline \multirow[b]{2}{*}{ SNP } & \multirow[b]{2}{*}{ genotype } & \multicolumn{2}{|r|}{ eGFR } & \multicolumn{3}{|c|}{$\mathrm{AGEs}^{\mathrm{a}}$} \\
\hline & & $n$ & & $n$ & CML & CEL \\
\hline \multirow{3}{*}{$\begin{array}{l}\text { SNP4 } \\
\text { (rs13199033) }\end{array}$} & $A A$ & 985 & $70.2 \pm 0.51$ & 433 & $1.76 \pm 0.02$ & $1.18 \pm 0.02$ \\
\hline & AT & 232 & $70.4 \pm 1.06$ & 105 & $1.84 \pm 0.05$ & $1.28 \pm 0.04$ \\
\hline & $\mathrm{TT}$ & 16 & $67.7 \pm 4.02$ & 7 & $1.67 \pm 0.18$ & $1.06 \pm 0.14$ \\
\hline \multirow{3}{*}{$\begin{array}{l}\text { SNP13 } \\
\text { (rs3799703) }\end{array}$} & GG & 388 & $68.5 \pm 0.82$ & 152 & $1.82 \pm 0.04$ & $1.22 \pm 0.03$ \\
\hline & $A G$ & 579 & $70.9 \pm 0.67$ & 256 & $1.71 \pm 0.03$ & $1.18 \pm 0.02$ \\
\hline & $A A$ & 271 & $69.5 \pm 0.97$ & 128 & $1.83 \pm 0.04$ & $1.21 \pm 0.03$ \\
\hline \multirow{3}{*}{$\begin{array}{l}\text { SNP16 } \\
\text { (rs2736655) }\end{array}$} & GG & 943 & $70.2 \pm 0.53$ & 407 & $1.77 \pm 0.02$ & $1.20 \pm 0.02$ \\
\hline & $A G$ & 280 & $70.0 \pm 0.96$ & 130 & $1.76 \pm 0.04$ & $1.20 \pm 0.04$ \\
\hline & $A A$ & 27 & $66.8 \pm 3.09$ & 11 & $2.03 \pm 0.14$ & $1.25 \pm 0.12$ \\
\hline \multirow{3}{*}{$\begin{array}{l}\text { SNP18 } \\
\text { (rs2736654) }\end{array}$} & $\mathrm{TT}$ & 381 & $69.3 \pm 0.82$ & 166 & $1.79 \pm 0.04$ & $1.23 \pm 0.03$ \\
\hline & GT & 616 & $70.9 \pm 0.65$ & 281 & $1.76 \pm 0.03$ & $1.16 \pm 0.02$ \\
\hline & GG & 258 & $69.1 \pm 1.00$ & 102 & $1.78 \pm 0.05$ & $1.23 \pm 0.04$ \\
\hline \multirow{3}{*}{$\begin{array}{l}\text { SNP28 } \\
\text { (rs3778443) }\end{array}$} & GG & 1108 & $69.6 \pm 0.48$ & 470 & $1.76 \pm 0.02$ & $1.20 \pm 0.02$ \\
\hline & $A G$ & 147 & $71.7 \pm 1.33$ & 77 & $1.82 \pm 0.05$ & $1.19 \pm 0.05$ \\
\hline & $A A$ & 7 & $64.3 \pm 6.03$ & 4 & $1.77 \pm 0.24$ & $1.22 \pm 0.20$ \\
\hline \multirow{3}{*}{$\begin{array}{l}\text { SNP38 } \\
\text { (rs1781716) }\end{array}$} & GG & 1062 & $69.4 \pm 0.49$ & 437 & $1.77 \pm 0.02$ & $1.20 \pm 0.02$ \\
\hline & CG & 191 & $71.6 \pm 1.16$ & 98 & $1.82 \pm 0.05$ & $1.19 \pm 0.04$ \\
\hline & $\mathrm{CC}$ & 8 & $64.2 \pm 5.64$ & 4 & $1.76 \pm 0.24$ & $1.23 \pm 0.20$ \\
\hline \multirow{3}{*}{$\begin{array}{l}\text { SNP40 } \\
\text { (rs10484854) }\end{array}$} & $\mathrm{CC}$ & 649 & $70.1 \pm 0.63$ & 280 & $1.79 \pm 0.03$ & $1.21 \pm 0.02$ \\
\hline & $\mathrm{CT}$ & 517 & $70.0 \pm 0.70$ & 228 & $1.75 \pm 0.03$ & $1.18 \pm 0.03$ \\
\hline & $\mathrm{TT}$ & 96 & $68.3 \pm 1.64$ & 39 & $1.78 \pm 0.08$ & $1.26 \pm 0.06$ \\
\hline \multirow{3}{*}{$\begin{array}{l}\text { SNP45 } \\
(\text { rs17622621) }\end{array}$} & GG & 463 & $70.0 \pm 0.75$ & 203 & $1.79 \pm 0.03$ & $1.18 \pm 0.03$ \\
\hline & $A G$ & 591 & $70.2 \pm 0.66$ & 257 & $1.74 \pm 0.03$ & $1.18 \pm 0.02$ \\
\hline & $A A$ & 180 & $68.6 \pm 1.20$ & 81 & $1.85 \pm 0.05$ & $1.28 \pm 0.04$ \\
\hline \multirow{3}{*}{$\begin{array}{l}\text { SNP49 } \\
\text { (rs1049346) }\end{array}$} & GG & 314 & $69.5 \pm 0.90$ & 120 & $1.77 \pm 0.04$ & $1.19 \pm 0.04$ \\
\hline & $A G$ & 650 & $70.2 \pm 0.63$ & 289 & $1.76 \pm 0.03$ & $1.19 \pm 0.02$ \\
\hline & $A A$ & 293 & $69.7 \pm 0.93$ & 140 & $1.82 \pm 0.04$ & $1.23 \pm 0.03$ \\
\hline
\end{tabular}

Data are expressed as mean \pm SE. eGFR, estimated glomerular filtration rate calculated by MDRD formula; CML, plasma levels of $\mathrm{N}^{\varepsilon}$ (carboxymethyl) lysine in $\mu \mathrm{mol} / \mathrm{L}$; CEL, plasma levels of $\mathrm{N}^{\varepsilon}$ (carboxyethyl) lysine $\mu \mathrm{mol} / \mathrm{L}$ ( ${ }^{a}$ data from the CODAM Study only). All analyses were adjusted for age, sex and glucose metabolism status. $P$-values for trends across genotypes all $>0.1$. 


\section{DISCUSSION}

We investigated whether nine common GLO1 gene SNPs were associated with markers of vascular and renal complications and plasma AGE levels in two Dutch cohorts. The nine GLO1 SNPs genotyped were in linkage disequilibrium $\left(r^{2}>0.8\right)$ with all 28 common (MAF $>5 \%)$ GLO1 SNPs, and therefore total common genetic variability of GLO1 was covered. Our results indicate that GLO1 gene polymorphisms were not associated with the prevalence of hypertension, markers of atherosclerosis, arterial stiffness or renal function. Although SNP18 (rs2736654) was weakly associated with PP and SNP40 (rs10484854) with SBP, both showing a protective effect in an additive model, these findings might be spurious due to the number of statistical tests performed.

An association between one of these common GLO1 SNPs, SNP18 (rs2736654), and plasminogen activator inhibitor-1 (PAI-1), has been previously reported. ${ }^{12}$ However, the effect size and the protective genotype were not reported, and therefore it remains unclear whether our results support or contradict those findings. In addition, the same GLO1 SNP was shown to be associated with cardio- and peripheral vascular disease in hemodialysis patients. ${ }^{13}$ However, in this study, the SNP was not in Hardy-Weinberg equilibrium and the increased prevalence of the vascular complications was attributed to the genotype that was found to be protective in our study (GG). These contradictory findings support the view of spurious associations mentioned above. No information on SNP40 (rs10484854) could be retrieved from the literature.

It was hypothesized that genetic variability in the GLO1 gene might lead to altered expression and/or activity of GLO1 and that this might alter AGE formation. We have therefore examined the associations between GLO1 gene polymorphisms with plasma levels of CML and CEL. No associations were found between the plasma concentration of CML and CEL and the nine common GLO1 gene SNPs. However, it might be possible that altered GLO1 expression and/or activity is not reflected by plasma levels of these AGEs, but rather by free methylglyoxal concentrations or intracellular AGE concentrations. Unfortunately, these measurements - which are complex - were not performed in the current study and we cannot rule out the possibility that other AGEs such as 5-hydro-5methylimidazolone, representing a major methylglyoxal-derived AGE in mammalian cells, ${ }^{7}$ may better reflect the GLO1 gene activity.

In conclusion, polymorphisms in the GLO1 gene are not associated with the prevalence of hypertension, markers of atherosclerosis, arterial stiffness, renal function and AGEs and are weakly associated with pulse pressure and systolic blood pressure (possibly due to chance) in two Dutch cohorts of subjects with normal glucose metabolism, impaired glucose metabolism, and type 2 diabetes. 


\section{REFERENCES}

1. Brownlee M. Biochemistry and molecular cell biology of diabetic complications. Nature. 2001;414(6865):813-820.

2. Goh SY, Cooper ME. The role of advanced glycation end products in progression and complications of diabetes. J Clin Endocrinol Metab. 2008;93(4):1143-1152.

3. Wang $\mathrm{X}$, Desai K, Chang T, Wu L. Vascular methylglyoxal metabolism and the development of hypertension. J Hypertens. 2005;23(8):1565-1573.

4. Wang $\mathrm{X}$, Jia $\mathrm{X}$, Chang T, Desai $\mathrm{K}, \mathrm{Wu}$ L. Attenuation of hypertension development by scavenging methylglyoxal in fructose-treated rats. J Hypertens. 2008;26(4):765-772.

5. Wu L, Juurlink BH. The impaired glutathione system and its up-regulation by sulforaphane in vascular smooth muscle cells from spontaneously hypertensive rats. J Hypertens. 2001;19(10):1819-1825.

6. Baynes JW, Thorpe SR. Role of oxidative stress in diabetic complications: a new perspective on an old paradigm. Diabetes. 1999;48(1):1-9.

7. Thornalley PJ. Glyoxalase I--structure, function and a critical role in the enzymatic defence against glycation. Biochem Soc Trans. 2003;31(Pt 6):1343-1348.

8. Shinohara M, Thornalley PJ, Giardino I, Beisswenger P, Thorpe SR, Onorato J, et al. Overexpression of glyoxalase-I in bovine endothelial cells inhibits intracellular advanced glycation endproduct formation and prevents hyperglycemia-induced increases in macromolecular endocytosis. J Clin Invest. 1998;101(5):11421147.

9. Kalapos MP. Methylglyoxal in living organisms: chemistry, biochemistry, toxicology and biological implications. Toxicol Lett. 1999;110(3):145-175.

10. Henry RM, Kostense PJ, Spijkerman AM, Dekker JM, Nijpels G, Heine RJ, et al. Arterial stiffness increases with deteriorating glucose tolerance status: the Hoorn Study. Circulation. 2003;107(16):2089-2095.

11. Kruijshoop M, Feskens EJ, Blaak EE, de Bruin TW. Validation of capillary glucose measurements to detect glucose intolerance or type 2 diabetes mellitus in the general population. Clin Chim Acta. 2004;341(1-2):3340.

12. Gale CP, Futers TS, Summers LK. Common polymorphisms in the glyoxalase-1 gene and their association with pro-thrombotic factors. Diab Vasc Dis Res. 2004;1(1):34-39.

13. Kalousova M, Germanova A, Jachymova M, Mestek O, Tesav V, Zima T. A419C (E111A) Polymorphism of the Glyoxalase I Gene and Vascular Complications in Chronic Hemodialysis Patients. Ann N Y Acad Sci. 2008;1126:268-271. 


\section{CHAPTER 7}

THE ASSOCIATION BETWEEN THE -37LT/A POLYMORPHISM OF THE RECEPTOR FOR ADVANCED SLYYCATION ENDPRODUCTS SEME AND BLOOD PRESSURE AMD ARTERIAL STIFFNESS IS MODFIED BY SLLUCOSE METABOLISY STATUS: THE HOORN AKD CODAM STUDIES

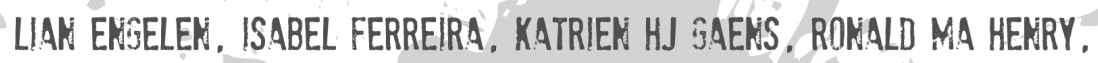
JACQUELINE M DEXXER, JIEL WIJPELS, ROBERT J HEINE, LEEK M T HART. MARLEEN MJ YAK ŚREEVENBROEX, CARLA JH YAN DER KaLLEN, ELLEN E BLAAK. EDITH JM FESKENS, HUSO TEN CATE, COEN DA STEHOUWER, CASPER SS SCHALKW'UK

J HYPERTENS. 2010 FEB:28[2]:285-93 


\section{ABSTRACT}

\section{Background}

Receptor for advanced glycation endproducts (RAGE)-ligand interaction may lead to vascular complications. Genetic variation in RAGE has been shown to alter expression and/or activity of RAGE. We therefore investigated whether RAGE single nucleotide polymorphisms (SNPs) and haplotypes were associated with vascular disease.

\section{Methods}

Nine tag SNPs that cover the common RAGE gene variation were genotyped in 1,291 individuals from two Dutch population-based cohort studies, aged $65 \pm 8$ years, with normal glucose metabolism (NGM; 44\%), impaired glucose metabolism (IGM; 23\%) or type 2 diabetes (T2DM; 33\%). We used multiple regression analyses to compare prevalent cardiovascular disease (CVD) and markers of atherosclerosis, blood pressure and arterial stiffness across genotypes, and examine effect modification by glucose metabolism status.

\section{Results}

In unstratified analyses, no consistent associations between RAGE SNPs and prevalent CVD and markers of atherosclerosis were found. However, the AA genotype of SNP rs1800624 $(-374 \mathrm{~T} / \mathrm{A})$ was consistently associated with lower systolic (SBP) $[-5.0 \mathrm{~mm} \mathrm{Hg}(95 \% \mathrm{Cl}$ : $10.4 ; 0.3)]$ and diastolic blood pressure (DBP) $[-4.2(-7.2 ;-1.3)]$, pulse pressure (PP) [-0.8 ($5.0 ; 3.4)]$ as well as with less arterial stiffness [-0.56 SD (-1.04;-0.09)] in individuals with NGM, but with higher SBP $[6.2(0.9 ; 11.5)]$, DBP $[2.1(-0.7 ; 5.0)]$ and PP $[4.1(-0.2 ; 8.4)]$ in individuals with IGM or T2DM ( $p$ for interaction $\leq 0.05$ in all analyses). Similar results were found for a haplotype that includes the $-374 \mathrm{~A}$ allele.

\section{Conclusion}

In individuals with NGM, the $-374 \mathrm{~A}$ allele of the RAGE gene is protectively, whereas in individuals with IGM or T2DM it is adversely associated with levels of blood pressure and not with arterial stiffness. 


\section{INTRODUCTION}

The mechanisms underlying the development of vascular complications in diabetes are not completely understood but increased formation of advanced glycation endproducts (AGEs) may constitute one such mechanism. ${ }^{1,2}$ The deleterious effects of AGEs on vascular structure and function are attributed to direct modification of extracellular matrix proteins, which results in collagen crosslinks, which may lead to increased vascular stiffness. ${ }^{1,2}$ In addition, binding of AGEs to a variety of cell-surface receptors, such as the receptor for advanced glycation endproducts (RAGE), triggers signal transduction pathways and the production of reactive oxygen species, resulting in proinflammatory cellular responses. ${ }^{3,4}$ It has been shown that RAGE is normally expressed at low levels in most tissues and is up-regulated in atherosclerotic lesions in individuals with diabetes. 6 The sustained interaction of RAGE with its ligands such as AGEs or S100 proteins increases the expression and activation of proinflammatory and procoagulant pathways, leading to vascular dysfunction. ${ }^{1,7}$

Genetic variation in the RAGE gene (i.e. AGER) has been shown to alter the expression and/or the activity of $\mathrm{RAGE}^{8,9}$ and may, as such, affect the genetic susceptibility to vascular disease. Studies so far investigating the association between RAGE polymorphisms [specifically rs1800624 (-374T/A), rs1800625 (-429T/C), rs2070600 (Gly82Ser) and rs184003 (+1704G/T)] and vascular disease have reported contradictory results (summarized in Table 7.1). These may have been due to differences in sample sizes and individuals' glucose metabolism status [i.e. type 1 (T1DM) or type 2 diabetes (T2DM) or normal glucose metabolism (NGM)] and/or the type of vascular disease studied.

In view of these considerations, we investigated in two population-based cohorts of Dutch individuals: 1 ) whether nine SNPs, which cover the entire common variability in the RAGE gene, were associated with prevalent cardiovascular disease and markers of atherosclerosis, blood pressure and arterial stiffness and 2) whether any such associations differed across individuals' glucose metabolism status (i.e. in those with NGM, IGM or T2DM). 
Table 7.1 Previously described associations of RAGE polymorphisms with vascular complications

\begin{tabular}{|c|c|c|c|c|c|c|}
\hline \multicolumn{2}{|c|}{ Ref.SNP } & \multirow{2}{*}{$\begin{array}{l}\text { Effect } \\
\text { None }\end{array}$} & \multirow{2}{*}{$\begin{array}{l}\text { Outcome } \\
\text { CAD }\end{array}$} & \multicolumn{3}{|c|}{ Sample size (n) Glucose metabolism Ethnicity } \\
\hline$\overline{24}$ & $-374 T / A$ & & & 1555 & N-DM/DM & Asian \\
\hline & & None & $\mathrm{DN}$ & 392 & T1DM & Caucasian \\
\hline & & None & In-stent restenosis & 297 & N-DM & Asian \\
\hline & & AA protective & Proteinuria & 118 & T1DM $\left(\mathrm{HbA}_{1 \mathrm{c}}>9.5\right)$ & Caucasian \\
\hline & & AA protective & CVD & 987 & T1DM & Caucasian \\
\hline & & AA protective & Stroke & 518 & N-DM & Caucasian \\
\hline & & None & MI & 682 & N-DM & Caucasian \\
\hline & & None & DR & 215 & T2DM & Caucasian \\
\hline & & AA protective & IHD & 131 & T2DM & African \\
\hline & & None & $\mathrm{DR}, \mathrm{DN}$ & 172,162 & T2DM & African \\
\hline 32 & & None & $\mathrm{DR}, \mathrm{DN}, \mathrm{IHD}$ & $476,453,346$ & T2DM & Caucasian \\
\hline & & AA protective & CV lesions & 98 & T2DM & Caucasian \\
\hline & & None & CV lesions & 148 & $\mathrm{~N}-\mathrm{DM}$ & Caucasian \\
\hline 4 & & None & DR & 357 & T2DM & Asian \\
\hline & & $\mathrm{TA} / \mathrm{AA}$ at risk, $\mathrm{TA} / \mathrm{AA}$ at risk, none & DR, DN, CVD & $694,444,751$ & T1DM & Caucasian \\
\hline 27 & & None & $\mathrm{DR}, \mathrm{DN}, \mathrm{CVD}$ & $877,471,1509$ & T2DM & Caucasian \\
\hline & & AA protective & DN & 184 & T2DM (low $\mathrm{HbA}_{1 \mathrm{c}}$ ) & Caucasian \\
\hline & & AA protective, none & Cardiac event & 521,122 & N-DM, T2DM & Caucasian \\
\hline & & AA protective & CAD severity & 234 & N-DM/T2DM & Caucasian \\
\hline & & None, none, AA at risk & $\mathrm{DR}, \mathrm{DN}, \mathrm{CVD}$ & $628,459,728$ & T1DM & Caucasian \\
\hline & & AA at risk, none, AA protective & $\mathrm{DR}, \mathrm{DN}, \mathrm{CVD}$ & $881,754,2697$ & T2DM & Caucasian \\
\hline 18 & & None & DR & 379 & T2DM & Asian \\
\hline 39 & & None & CAD & 409 & T2DM & Caucasian \\
\hline & & None & DR & 186 & T2DM & Caucasian \\
\hline 4 & $-429 \mathrm{~T} / \mathrm{C}$ & None & CAD & 1555 & N-DM/DM & Asian \\
\hline & & CC protective & MI & 682 & N-DM & Caucasian \\
\hline 2 & & None & Stroke & 518 & $\mathrm{~N}-\mathrm{DM}$ & Caucasian \\
\hline & & $\mathrm{TC} / \mathrm{CC}$ at risk & DR & 215 & T2DM & Caucasian \\
\hline 88 & & None & DN, CVD & 996 & T1DM & Caucasian \\
\hline & & None & $\mathrm{DR}, \mathrm{DN}, \mathrm{IHD}$ & $172,162,131$ & T2DM & African \\
\hline & & None & $\mathrm{DR}, \mathrm{DN}, \mathrm{IHD}$ & $476,453,346$ & T2DM & Caucasian \\
\hline 34 & & None & DR & 357 & T2DM & Asian \\
\hline 3 & & None & DR & 379 & T2DM & Asian \\
\hline 9 & & None & $C A D$ & 409 & T2DM & Caucasian \\
\hline & & None & DR & 186 & T2DM & Caucasian \\
\hline 30 & Gly82Ser & None & DN & 392 & T1DM & Caucasian \\
\hline 1 & & None & CVD & 1632 & N-DM/DM & Caucasian \\
\hline 2 & & None & MA & 348 & T2DM & Asian \\
\hline 13 & & None & DR & 268 & T2DM & Asian \\
\hline & & None & $\mathrm{DN}, \mathrm{CVD}$ & 996 & T1DM & Caucasian \\
\hline & & None & MI, stroke & 682,518 & N-DM & Caucasian \\
\hline 14 & & None & $\mathrm{CHD}$ & 596 & N-DM/DM & Caucasian \\
\hline 15 & & $\mathrm{GA} / \mathrm{AA}$ at risk & DN & 487 & T1DM & Caucasian \\
\hline 46 & & GA/AA protective & DR & 200 & T2DM & Asian \\
\hline 7 & & None & $\mathrm{DR}, \mathrm{DN}$ & 156 & T2DM & Asian \\
\hline 7 & & GA/AA protective & CAD & 1555 & N-DM/DM & Asian \\
\hline & $+1704 \mathrm{G} / \mathrm{T}$ & TNone & $\mathrm{MA}$ & 348 & T2DM & Asian \\
\hline & & None & DR & 268 & T2DM & Asian \\
\hline & & $\mathrm{GT} / \mathrm{TT}$ at risk & DN & 181 & T2DM & Asian \\
\hline & & None & DR & 287 & T2DM & Caucasian \\
\hline
\end{tabular}

$S N P$, single nucleotide polymorphism; $N-D M$, non-diabetes mellitus; T1DM, type 1 diabetes mellitus; T2DM, type 2 diabetes mellitus; $C A D$, coronary artery disease; $D N$, diabetic nephropathy; $D R$, diabetic retinopathy; $C V(D)$, cardiovascular (disease); $M I$, myocardial infarction; $I H D$, ischaemic heart disease; $M A$, microalbuminuria. 


\section{METHODS}

\section{STUDY POPULATION}

The present study included participants from the follow-up examination of the Hoorn Study ${ }^{10,11}$ and from the Cohort Diabetes and Atherosclerosis Maastricht (CODAM) study. ${ }^{12-}$ 14

Briefly, the Hoorn study, which started in 1989 , is a population-based cohort study investigating glucose tolerance status and cardiovascular disease risk factors among a sample of the general population of Hoorn, the Netherlands. In 2000-2001, a follow-up examination was conducted in a selected group of 822 subjects consisting of a random sample of subjects with NGM or IGM and all subjects with T2DM of the survival cohort and 188 additional subjects with T2DM from the Hoorn Screening Study. ${ }^{15}$ Details of the study design have been described previously. ${ }^{10,11}$ The CODAM Study started in 1999-2000 and is a cohort study designed to investigate the effects of glucose metabolism, blood lipids, lifestyle, and genetic factors on cardiovascular disease morbidity and mortality. After a first screening oral glucose tolerance test, subjects from a large population-based cohort ${ }^{16}$ were invited to participate in the study on the basis of the following inclusion criteria: Caucasian ethnicity, age $>40$ years, and at least one of the following: body mass index (BMI; in $\mathrm{kg} / \mathrm{m}^{2}$ ) $>25$, or family history of T2DM, history of gestational diabetes, use of antihypertensive medication, postprandial blood glucose concentration $>6.0 \mathrm{mmol} / \mathrm{L}$, or glucosuria. In total, 574 subjects were enrolled. ${ }^{12-14}$

Both cohort studies were approved by the local Ethics Committees (Ethical Review Committee of the VU University Medical Center Amsterdam for the Hoorn Study; Medical Ethical Review Committee of Maastricht University Medical Centre for the CoDAM study). Written informed consent was obtained from all participants.

Individuals who had missing data in all genotyped SNPs (mainly due to missing DNA samples, $n=105$; 101 from Hoorn; 4 from CODAM) were excluded from the analyses. These individuals did not differ from those included with regard to the main outcomes and other covariates investigated (data not shown). The final study population therefore consisted of 1,291 subjects ( 721 from Hoorn, 570 from CODAM), $44 \%$ of whom had NGM, $23 \%$ had IGM and 33\% had T2DM. ${ }^{17}$ 


\section{SNP SELECTION AND GENOTYPING}

In order to enable coverage of the total RAGE gene, including the promoter region and the 5' untranslated region or the 3' untranslated region, SNP selection was based on the RAGE gene including 3000 base pairs downstream and upstream. Using the HapMap database (HAPMAP phase 2, January 2006, population CEU), we identified ten SNPs with a Minor Allele Frequency $(\mathrm{MAF})>1 \%$. Using the tagger option in Haploview, we selected six tagSNPs for genotyping using a Linkage Disequilibrium (LD) of $r^{2}>0.8$. These tag SNPs, which cover the common variation of the RAGE gene, included rs204995, rs1800625 (-429T/C), rs1035798, rs3134943, rs9469089 and rs3134945. In addition, we selected SNP rs1800624 (-374T/A), rs2070600 (Gly82Ser) and rs184003 (+1704G/T) for their potential functional effects on the basis of earlier findings reported in the literature. In total, nine polymorphisms were genotyped. ${ }^{18}$ The gene structure of RAGE including the RAGE polymorphisms, their locations, and sequence changes are shown in Figure 7.1.

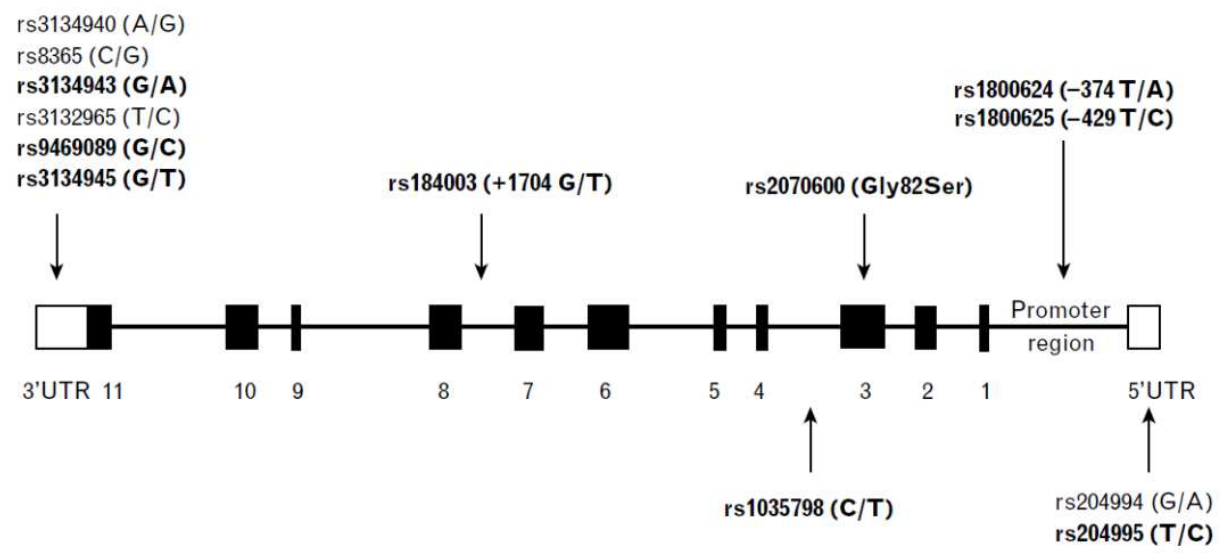

Figure 7.1 Representation of the human RAGE gene

Arrows indicate the approximate locations of the common SNPs in the RAGE gene; the nine SNPs genotyped in this study are printed in bold. Black boxes represent exons 1-11 and promotor and intron sequences are indicated as horizontal lines. White boxes represent the $3^{\prime}$ and $5^{\prime}$ untranslated regions.

For genotyping, DNA was isolated from peripheral blood leucocytes (obtained from EDTA anticoagulated blood) by a standard DNA extraction method. SNP rs204995, rs2070600, rs1035798, rs184003, rs3134943, rs9469089 and rs3134945 were genotyped using the ABI PRISM 7900HT Sequence Detection System (Applied Biosystems, Foster City, CA) in a $5 \mu \mathrm{L}$ reaction according to the manufacturer's instructions. Because of the presence of a $63 \mathrm{bp}$ insertion/deletion close to SNPs rs1800624 and rs1800625, no assay could be developed 
for Taqman genotyping. These promoter SNPs were therefore determined together with the -407 to -345 (63 bp) deletion from a single polymerase chain reaction (PCR) using the primers 5'-AAAAACATGAGAAACCCCAGAA-3' and 5'-AGAGCCCCCGATCCTATTTA-3'. After amplification, the products (228 bp) were digested with the restriction endonucleases Alul and Mfel and run on an ethidium-bromide stained $4 \%$ agarose gel. Ninety-three percent of the genotyping analyses were successful for all SNPs. ${ }^{18}$

\section{PREVALENT CARDIOVASCULAR DISEASE AND MARKERS OF ATHEROSCLEROSIS, BLOOD PRESSURE AND ARTERIAL STIFFNESS}

Prevalent cardiovascular disease (CVD) was defined as a history of myocardial infarction, stroke or transient ischemic attack, non-traumatic limb amputation, coronary or peripheral artery bypass, angioplasty, or signs of infarction or ischemia on a 12-lead electrocardiogram (Minnesota codes 1-1, 1-2, 1-3, 4-1, 4-2, 4-3, 5-1, 5-2, 5-3 or 7-1) or an ankle-brachial index $(\mathrm{ABI})<0.9$ in either leg. ${ }^{11,14}$

Systolic (SBP) and diastolic (DBP) blood pressures were measured using an oscillometric device (Press-Mate, BP-8800; Colin Medical Instruments Corp., San Antonio, Texas, USA, in the Hoorn Study) or a high precision aneroid sphygmomanometer (Speidel \& Kelly Maxi Stabil 3, Jungingen, Germany, in the CoDAM study). Hypertension was defined as SBP $\geq 140 \mathrm{~mm} \mathrm{Hg}$ and/or DBP $\geq 90 \mathrm{~mm} \mathrm{Hg}$ and/or use of blood pressure lowering medication. ${ }^{19}$

$A B I$ and carotid intima-media thickness (CIMT) were used as markers of atherosclerosis. Doppler assisted measurements of SBP in the brachial and posterior tibial and dorsalis pedis arteries (both sides) were performed with subjects in the supine position. The $A B I$ was calculated for each leg by dividing the highest ankle pressure by the highest brachial pressure. The lowest $A B I$ of either leg was used in the analyses. Subjects with $A B I$ values $>1.5$ were excluded from the analyses $(n=5)$. Non-invasive ultrasound imaging techniques and devices (Pie 350 Series; Pie Medical BV, Maastricht, the Netherlands in the Hoorn study and Ultramark 4+, Advance Technology Laboratories, Bothel, Washington, USA in the CoDAM study) were used to measure large artery properties according to similar protocols in both studies, ${ }^{11}$ which comply with international guidelines for user procedures. ${ }^{20}$ Briefly, CIMT was measured in the right common carotid artery and, in addition, but in the Hoorn study only, diameter and distension were obtained from the right common carotid, femoral and brachial arteries. These measurements, in combination with local pulse pressures (PP), were used to calculate the following stiffness estimates: the distensibility coefficient (DC, which reflects the elastic properties) and the compliance coefficient (CC, which reflects the buffering capacity) of the three arteries, and the carotid Young's elastic modulus (YEM, an estimate 
of the intrinsic elastic properties of the vessel wall). ${ }^{11}$ Each of the stiffness estimates (i.e. DC, CC and YEM (multiplied by -1)) was adjusted for subjects' level of MAP and height prior to further analyses. These adjusted values were then standardized (subjects' value population mean / SD) and compiled into an overall sex-specific arterial stiffness Z-score, so that a higher score reflects greater stiffness. Similarly, separate stiffness scores for the elastic (carotid) and the muscular (femoral and brachial) arteries were computed.

\section{BLOOD AND URINE SAMPLES AND BIOCHEMICAL MEASUREMENTS}

Fasting venous blood samples were drawn and serum and plasma were immediately separated after centrifugation and stored at $-80^{\circ} \mathrm{C}$ until assays were performed. ${ }^{11-13}$

Renal function was described by glomerular filtration rate (eGFR, in $\mathrm{mL} / \mathrm{min} / 1.73 \mathrm{~m}^{2}$ ) estimated with the use of the short MDRD formula ([186* $\left((\text { creatinine/88.4) })^{-1.154}\right) *$ (age $\left.\left.{ }^{0.203}\right)\right] * 0.742$ if female). ${ }^{21}$

Morning samples of urine were frozen at $-20^{\circ} \mathrm{C}$. The albumin concentration was determined by rate nephelometry (Array Protein System, Beckman Inc., Brea, California, USA). Microalbuminuria was considered to be present when albumin concentration was 20-200 $\mathrm{mg} / \mathrm{L}$ and macroalbuminuria was considered to be present when albumin concentration was $>200 \mathrm{mg} / \mathrm{L}^{22}$

\section{STATISTICAL ANALYSES}

Statistical analyses were carried out using the Statistical Package for Social Sciences, version 15.0 (SPSS Inc., Chicago, IL, USA). Hardy-Weinberg equilibrium was assessed using a $\chi^{2}$ test. We compared the genotype frequencies of all SNPs across the NGM, IGM and T2DM groups and the two study populations with $\chi^{2}$ tests. Linear and logistic regression analyses were used to compare outcome variables across genotypes of the nine RAGE gene polymorphisms. We used dominant- (using major homozygous genotype as a reference), recessive- (using major homozygous plus heterozygous genotype as a reference) and additive models (comparison across three genotypes) in these analyses. We have also carried out analyses with haplotypes, which were constructed according to two haplotype blocks with the use of Haploview and the SNPHAP program gene.cimr.cam.ac.uk/clayton/software) and combined either SNPs rs1800624 (-374T/A), rs1800625 (-429T/C), rs2070600 (Gly82Ser) and rs184003 (+1704G/T) (previously described in literature) or SNPs rs3134943, rs9469089 and rs3134945. For each haplotype, we compared study outcomes between the homozygous $+/+$, the heterozygous $+/-$ and the homozygous -/- (e.g. ATCG/ATCG, ATCG/other and other/other, respectively), using 
linear and logistic regression analyses similar to those described in the single SNP analyses. Haplotypes were only assigned when the probability was $>95 \%$ based on the SNPHAP program and haplotypes with a frequency of $<0.5 \%$ were dropped from the analyses.

Table 7.2 Characteristics of the Hoorn study, CODAM study and the total population

\begin{tabular}{|c|c|c|c|}
\hline & Hoorn & CODAM & Total population \\
\hline$n(\mathrm{~m} / \mathrm{f})$ & $721(361 / 360)$ & $570(349 / 221)$ & $1291(710 / 581)$ \\
\hline Age (years) & $68.8 \pm 7.2$ & $59.1 \pm 6.9$ & $64.5 \pm 8.6$ \\
\hline BMI $\left(\mathrm{kg} / \mathrm{m}^{2}\right)$ & $27.7 \pm 4.1$ & $28.6 \pm 4.3$ & $28.1 \pm 4.2$ \\
\hline Waist circumference $(\mathrm{cm})$ & $96.6 \pm 12.1$ & $99.4 \pm 11.9$ & $97.8 \pm 12.1$ \\
\hline NGM/IGM/T2DM (\%) & $36 / 24 / 40$ & $53 / 22 / 25$ & $44 / 23 / 33$ \\
\hline Fasting glucose (mmol/L) & $6.49 \pm 1.55$ & $6.08 \pm 1.48$ & $6.31 \pm 1.54$ \\
\hline Postload glucose (mmol/L) & $7.27 \pm 2.60$ & $7.73 \pm 3.50$ & $7.50 \pm 3.10$ \\
\hline Glycated hemoglobin (\%) & $6.11 \pm 0.77$ & $5.97 \pm 0.82$ & $6.05 \pm 0.80$ \\
\hline Fasting insulin (pmol/L) & $61(43-91)$ & $60(45-89)$ & $60(44-90)$ \\
\hline HOMA-IR & $1.2(0.8-1.8)$ & $1.2(0.8-1.7)$ & $1.2(0.9-1.8)$ \\
\hline Total cholesterol (mmol/L) & $5.67 \pm 1.05$ & $5.22 \pm 0.99$ & $5.47 \pm 1.04$ \\
\hline HDL-cholesterol (mmol/L) & $1.38 \pm 0.40$ & $1.19 \pm 0.35$ & $1.30 \pm 0.39$ \\
\hline LDL-cholesterol (mmol/L) & $3.58 \pm 0.92$ & $3.31 \pm 0.86$ & $3.46 \pm 0.90$ \\
\hline Triglycerides (mmol/L) & $1.58 \pm 0.89$ & $1.65 \pm 1.55$ & $1.61 \pm 1.22$ \\
\hline Prevalent cardiovascular disease (\%) & 48.5 & 27.7 & 39.1 \\
\hline \multicolumn{4}{|l|}{ Blood pressure } \\
\hline Systolic blood pressure (mm Hg) & $143 \pm 20.3$ & $140 \pm 19.1$ & $142 \pm 19.8$ \\
\hline Diastolic blood pressure (mm Hg) & $83.2 \pm 10.9$ & $81.9 \pm 9.2$ & $82.6 \pm 10.2$ \\
\hline Mean arterial blood pressure $(\mathrm{mm} \mathrm{Hg})$ & $103 \pm 12.4$ & $101 \pm 11.4$ & $102 \pm 12.0$ \\
\hline Pulse pressure $(\mathrm{mm} \mathrm{Hg})$ & $59.5 \pm 16.8$ & $58.5 \pm 14.7$ & $59.1 \pm 16.0$ \\
\hline Hypertension (\%) & 71.5 & 62.6 & 67.6 \\
\hline \multicolumn{4}{|l|}{ Markers of atherosclerosis } \\
\hline Carotid intima-media thickness (mm) & $0.86 \pm 0.17$ & $0.78 \pm 0.16$ & $0.82 \pm 0.17$ \\
\hline Ankle-brachial index & $1.16 \pm 0.14$ & $1.15 \pm 0.13$ & $1.16 \pm 0.14$ \\
\hline \multicolumn{4}{|l|}{ Markers of arterial stiffness } \\
\hline Carotid DC $\left(10^{-3} \mathrm{kPa}^{-1}\right)$ & $11.5 \pm 4.36$ & - & \\
\hline Carotid CC $\left(\mathrm{mm}^{2} \mathrm{kPa}^{-1}\right)$ & $0.56 \pm 0.23$ & - & \\
\hline Carotid YEM $\left(10^{3} \mathrm{kPa}\right)$ & $0.98 \pm 0.52$ & - & \\
\hline Femoral DC $\left(10^{-3} \mathrm{kPa}^{-1}\right)$ & $4.65 \pm 2.11$ & - & \\
\hline Femoral CC $\left(\mathrm{mm}^{2} \mathrm{kPa}^{-1}\right)$ & $0.36 \pm 0.19$ & - & \\
\hline Brachial DC $\left(10^{-3} \mathrm{kPa}^{-1}\right)$ & $7.52 \pm 4.00$ & - & \\
\hline Brachial CC $\left(\mathrm{mm}^{2} \mathrm{kPa}^{-1}\right)$ & $0.13 \pm 0.07$ & - & \\
\hline \multicolumn{4}{|l|}{ Markers of renal disease } \\
\hline Albuminuria: normo/micro/macro (\%) & $87 / 11.5 / 1.5$ & $92.4 / 6.5 / 1.1$ & 89.3/9.4/1.3 \\
\hline eGFR (mL/min $/ 1.73 \mathrm{~m}^{2}$ body surface area) & $57.4 \pm 9.6$ & $85.7 \pm 17.0$ & $69.9 \pm 19.5$ \\
\hline \multicolumn{4}{|l|}{ Use of medication } \\
\hline Glucose lowering medication (\%) & 8.2 & 13.3 & 10.5 \\
\hline Blood pressure lowering medication (\%) & 39.1 & 38.4 & 38.8 \\
\hline Lipid lowering medication (\%) & 16.7 & 18.9 & 17.7 \\
\hline
\end{tabular}

Data are expressed as mean \pm SD, median (inter-quartile range) or percentages. NGM, normal glucose metabolism; IGM, impaired glucose metabolism; T2DM, type 2 diabetes mellitus; HOMA-IR, insulin resistance based on the homeostasis model of assessment; $D C$, distensibility coefficient; $C C$, compliance coefficient; YEM, Young's elastic modulus; eGFR estimated glomerular filtration rate calculated by MDRD formula. 
All analyses were first adjusted for age, sex, glucose metabolism status, study population and use of blood pressure-, lipid- and glucose-lowering medication. After we had assessed the main effects, we added interaction terms between the RAGE polymorphisms and glucose metabolism status. Whenever significant interactions were found (i.e. $p<0.1$ ), data were stratified according to glucose metabolism status; because the interactions found concerned differences between NGM and both IGM and T2DM, these two latter groups were merged in these analyses, thereby also increasing the power of these analyses.

\section{RESULTS}

Baseline characteristics are shown in Table 7.2. Compared with individuals from the CODAM study, the Hoorn study population was older, included fewer men but more individuals with type 2 diabetes and hypertension, and had higher levels of cholesterol, PP and CIMT.

All genotyped SNPs were in Hardy-Weinberg equilibrium. Genotype frequencies did not differ between the NGM, IGM and the T2DM ( $p>0.18$ for all) individuals, but between both study populations we found differences in genotype frequencies of SNPs rs 1800624 (-374 T/A), rs1800625 (-429 T/C), rs1035798 and rs3134945 (Table 7.3).

\section{ASSOCIATIONS BETWEEN RAGE SNPS AND PREVALENT CVD AND MARKERS OF ATHEROSCLEROSIS, BLOOD PRESSURE AND ARTERIAL STIFFNESS}

Prevalence of hypertension and albuminuria did not differ across genotypes of the nine RAGE SNPs in the whole study population (data not shown). We found several significant associations between RAGE SNPs and markers of atherosclerosis, blood pressure and arterial stiffness in the whole population, but all these associations differed across glucose metabolism status (NGM vs IGM or T2DM; $p$ for interaction $\leq 0.09$ for all analyses. Therefore, we hereby report the results on these analyses stratified according to glucose metabolism (NGM vs. IGM or T2DM). In both the NGM and IGM/T2DM groups, most SNPs were inconsistently associated with prevalent CVD and markers of atherosclerosis, blood pressure and arterial stiffness (Tables A7.1-A7.3, Appendix).

However, in individuals with NGM and using recessive models, we found that the AA genotype of SNP rs1800624 (-374T/A) was associated with lower SBP [-5.0 mm Hg $(95 \% \mathrm{Cl}$ : $-10.4 ; 0.3)]$, lower DBP $[-4.2 \mathrm{~mm} \mathrm{Hg}(-7.2 ;-1.3)]$, lower PP $[-0.8 \mathrm{~mm} \mathrm{Hg}(-5.0 ; 3.4)]$ as well as less arterial stiffness $[-0.56$ SD $(-1.04 ;-0.09)]$. Similar results were found for the separate stiffness scores of elastic $[-0.54$ SD $(-1.01 ;-0.06)]$ and muscular $[-0.58$ SD (-1.06; - 
0.10)] arteries. In contrast, in individuals with IGM or $T 2 D M$, the AA genotype was associated with higher SBP [6.2 mm Hg (0.9; 11.5)], higher DBP [2.1 mm Hg (-0.7; 5.0)], higher PP $[4.1 \mathrm{~mm} \mathrm{Hg}(-0.2 ; 8.4)]$, but not with total arterial stiffness [0.10 SD $(95 \% \mathrm{Cl}$ : $0.27 ; 0.46)]$, elastic- [0.09 SD $(-0.28 ; 0.46)]$ or muscular [0.11 SD $(-0.26 ; 0.47)]$ stiffness (Figure 7.2).

In addition, SNP rs1035798, which is in LD $\left(r^{2}=0.935\right)$ with SNP rs1800624 (-374T/A), showed a similar pattern of associations with blood pressure and arterial stiffness (i.e. the TT genotype was associated with lower blood pressure and arterial stiffness in individuals with $N G M$, but with higher blood pressure and arterial stiffness in individuals with IGM or T2DM, Table A7.1).

Table 7.3 Genotype frequencies of RAGE SNPs for both study populations

\begin{tabular}{|c|c|c|c|c|}
\hline \multirow{2}{*}{ SNP } & \multirow{2}{*}{ genotype } & \multicolumn{2}{|c|}{$n(\%)$} & \multirow{2}{*}{$P$-value ${ }^{a}$} \\
\hline & & Hoorn & CODAM & \\
\hline \multirow{3}{*}{ rs204995 } & TT & $413(59.3)$ & 306 (58.1) & \multirow{3}{*}{0.124} \\
\hline & TC & $240(34.4)$ & $200(38.0)$ & \\
\hline & CC & $44(6.3)$ & $21(4.0)$ & \\
\hline \multirow{3}{*}{$\begin{array}{l}\text { rs1800624 } \\
\text { (-374T/A) }\end{array}$} & TT & $367(53.7)$ & $249(45.4)$ & \multirow{3}{*}{0.012} \\
\hline & TA & 269 (39.3) & 247 (45.1) & \\
\hline & AA & $48(7.0)$ & $52(9.5)$ & \\
\hline \multirow{3}{*}{$\begin{array}{l}\text { rs1800625 } \\
(-429 T / C)\end{array}$} & TT & $453(65.2)$ & $359(65.5)$ & \multirow{3}{*}{$<0.001$} \\
\hline & TC & 208 (29.9) & $185(33.8)$ & \\
\hline & CC & $34(4.9)$ & $4(0.7)$ & \\
\hline \multirow{3}{*}{$\begin{array}{l}\text { rs2070600 } \\
\text { (Gly82Ser) }\end{array}$} & CC & $675(94.4)$ & $493(93.4)$ & \multirow{3}{*}{0.747} \\
\hline & CT & $39(5.5)$ & $34(6.4)$ & \\
\hline & TT & $1(0.1)$ & $1(0.2)$ & \\
\hline \multirow{3}{*}{ rs1035798 } & CC & $380(54.1)$ & $243(46.1)$ & \multirow{3}{*}{0.019} \\
\hline & CT & 275 (39.1) & $237(45.0)$ & \\
\hline & TT & $48(6.8)$ & 47 (8.9) & \\
\hline \multirow{3}{*}{$\begin{array}{l}\text { rs184003 } \\
(+1704 G / T)\end{array}$} & GG & $627(87.8)$ & $457(85.1)$ & \multirow{3}{*}{0.374} \\
\hline & GT & 83 (11.6) & 76 (14.2) & \\
\hline & TT & $4(0.6)$ & $4(0.7)$ & \\
\hline \multirow{3}{*}{ rs3134943 } & GG & $533(74.5)$ & 420 (78.1) & \multirow{3}{*}{0.282} \\
\hline & GA & $173(24.2)$ & $110(20.4)$ & \\
\hline & AA & $9(1.3)$ & $8(1.5)$ & \\
\hline \multirow{3}{*}{ rs9469089 } & GG & $493(69.1)$ & 385 (72.1) & \multirow{3}{*}{0.526} \\
\hline & GC & $194(27.2)$ & $131(24.5)$ & \\
\hline & $\mathrm{CC}$ & $26(3.6)$ & $18(3.4)$ & \\
\hline \multirow{3}{*}{ rs3134945 } & GG & $432(60.9)$ & $323(61.3)$ & \multirow{3}{*}{0.009} \\
\hline & GT & 238 (33.6) & $193(36.6)$ & \\
\hline & TT & 39 (5.5) & $11(2.1)$ & \\
\hline
\end{tabular}

${ }^{a} P$-values for difference in genotype frequencies between both study populations were determined using a $\chi^{2}$ test. 

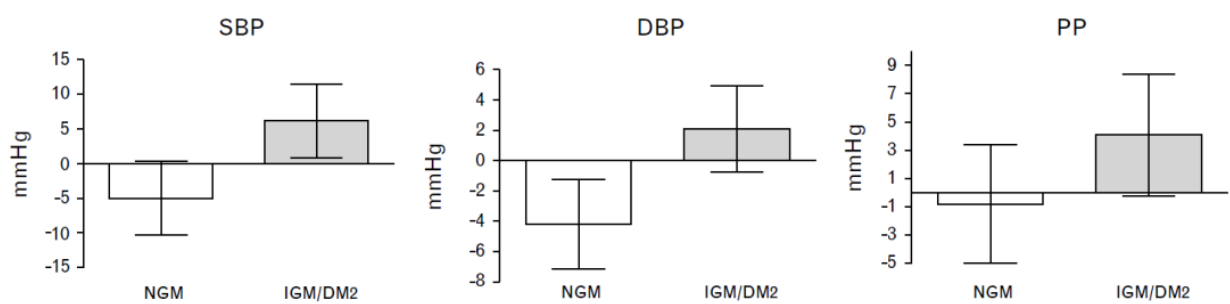

TS

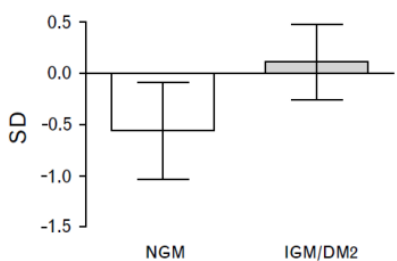

ES
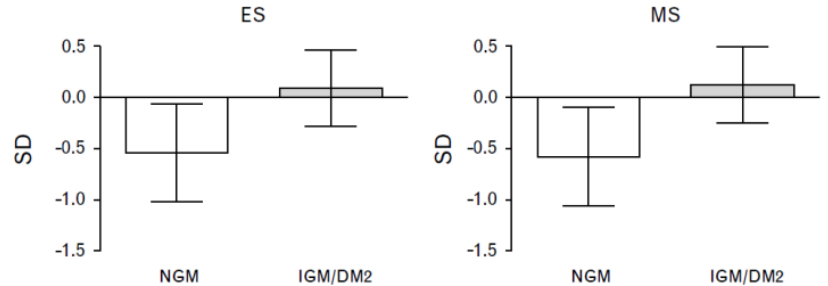

Figure 7.2 Associations of RAGE SNP rs1800624 (-374T/A) with levels of blood pressure and arterial stiffness Bars indicate differences in blood pressure (in $\mathrm{mm} \mathrm{Hg}$ ) or arterial stiffness (in SD) $\pm 95 \% \mathrm{Cl}$ between genotypes as estimated by recessive models (i.e. AA vs. TT/TA). TS, ES, and MS, total, elastic and muscular arterial stiffness scores, respectively. All analyses were adjusted for age, sex, study population and use of blood pressure-, lipid- and glucoselowering medication.

\section{ASSOCIATIONS BETWEEN RAGE HAPLOTYPES AND PREVALENT CVD AND MARKERS OF ATHEROSCLEROSIS, BLOOD PRESSURE AND ARTERIAL} STIFFNESS

The haplotypes constructed on the basis of the four SNPs rs1800624 (-374T/A), rs1800625 $(-429 \mathrm{~T} / \mathrm{C})$, rs2070600 (Gly82Ser, C/T) and rs184003 (+1704G/T), showed that using recessive models in individuals with $N G M$, the ATCG haplotype (including the -374A allele, frequency: 7.8\%) was associated with lower SBP $[-4.7 \mathrm{~mm} \mathrm{Hg}(-10.5 ; 1.1)]$, DBP [-4.5 mm $\mathrm{Hg}(-7.6 ;-1.4)], \mathrm{PP}[-0.2 \mathrm{~mm} \mathrm{Hg}(-2.1 ; 1.7)]$ as well as less arterial stiffness [-0.58 SD $(-1.06$; $-0.10)]$. Similar to the single SNP analyses, using recessive models in individuals with IGM or T2DM, this haplotype (frequency: $8.1 \%$ ) was associated with higher SBP [6.5 mm Hg (1.3; 11.8)], DBP [2.3 mm Hg $(-0.6 ; 5.2)]$ and PP [1.4 $\mathrm{mm} \mathrm{Hg}(-0.5 ; 3.2)]$, but not with arterial stiffness [0.08 SD $(-0.29 ; 0.45)]$.

The haplotypes constructed on the basis of the three SNPs rs3134943, rs9469089, and rs3134945 were inconsistently associated with study outcomes (data not shown). 


\section{DISCUSSION}

RAGE-ligand interaction triggers signal transduction pathways, resulting in proinflammatory cellular responses, and when sustained leading to micro- and macrovascular dysfunction. In the present study, we investigated whether nine RAGE gene polymorphisms and haplotypes, which covered the common genetic variability of RAGE, were associated with prevalent CVD and markers of atherosclerosis, blood pressure and arterial stiffness in a large cohort of Dutch individuals with different glucose metabolism status. We found that the AA genotype of SNP rs1800624 (-374T/A) was associated with lower SBP, DBP, PP, and less arterial stiffness in individuals with $N G M$, but with higher levels of these variables in individuals with IGM or T2DM. Similar results were found for a haplotype that includes the $-374 \mathrm{~A}$ allele. Associations between the other RAGE polymorphisms and prevalent CVD and markers of atherosclerosis, blood pressure and arterial stiffness were inconsistent, however.

Associations between several RAGE polymorphisms and vascular complications, such as cardiovascular disease, stroke, ischaemic heart disease, nephropathy, retinopathy and myocardial infarction have often been contradictory (Table 7.1). In addition, several RAGE haplotype studies, combining the four common SNPs have also shown associations with diabetic nephropathy (haplotype including $-429 \mathrm{C}$ allele at risk), ${ }^{23}$ coronary artery disease (haplotype including $82 \mathrm{Ser}$ allele protective), ${ }^{24}$ myocardial infarction and stroke (haplotypes including $-429 \mathrm{C}$ or $-374 \mathrm{~A}$ allele protective). ${ }^{25}$ In one study population of haemodialysis patients, however, no association was found of these haplotypes with cardiovascular disease, peripheral vascular disease or hypertension. ${ }^{26}$ These contradictory findings in associations of RAGE polymorphisms and haplotypes with cardiovascular disease may have been due to differences in the sample sizes, the ethnicity of the population studied and the glucose metabolism status of the subjects. The modifying effect of glucose metabolism status is supported by our current findings obtained in a rather large population $(n=1,291)$ of Caucasian subjects showing that associations between RAGE gene polymorphisms differed across different status of glucose metabolism.

We found a protective role in terms of blood pressure and arterial stiffness of the AA genotype of SNP rs1800624 (-374T/A) in individuals with NGM, but a deleterious (for blood pressure) or no effect (for arterial stiffness) in individuals with IGM/T2DM. Differences in the associations of this polymorphism with renal disease according to individuals' levels of glycaemic control have been previously described in individuals with type $1^{27}$ and type 2 diabetes, ${ }^{28}$ although the modifying effect of glycaemic control reported in those studies was contradictory. Lindholm et al. ${ }^{27}$ found a protective effect of 
the AA genotype for renal disease confined to individuals with low $\mathrm{HbA}_{1 \mathrm{c}}$, whereas Petterson-Fernholm et al. $^{28}$ described a protective role of the same allele only in individuals with poor metabolic control (i.e. high $\mathrm{HbA}_{1 \mathrm{c}}$ ).

In view of our results and previously published data, it seems that SNPs associated with the development of vascular disease are all functional SNPs or in LD with functional SNPs. The SNPs rs1800624 (-374T/A) and rs1800625 (-429T/C) are located in the promoter region and have been shown to increase reporter gene expression ${ }^{9}$ and the functional rs2070600 polymorphism (Gly82Ser) occurs in the ligand-binding V-domain of RAGE and affects ligand affinity. ${ }^{8}$ However, the fact that the association between the $-374 T / A$ polymorphism and blood pressure and arterial stiffness differed according to glucose metabolism status suggests that the deleterious effects of the higher glucose levels or other metabolic abnormalities associated with glucose intolerance, may influence the protective or deleterious effects of RAGE polymorphisms on cardiovascular disease. Although further studies are required to explain this effect, it might be that the nuclear binding factor, of which binding may be prevented by the $T>A$ substitution at position 374 , thereby influencing repression of RAGE transcription, ${ }^{9,2}$ is glucose-dependent.

A limitation to our study might be the large number of statistical tests we performed, rising the possibility of chance findings. Nevertheless, we could confirm our findings with regard to the association between SNP rs1800624 (-374T/A) and blood pressure in both study populations separately, or when the IGM and the T2DM groups were analyzed separately, which were similar in magnitude and direction (though with less power, given the lower sample size in sub-group analyses). In addition, although we found similar patterns of associations between SNP rs1800624 (-374T/A) and eGFR, these were not found with regard to other blood pressure related outcomes such as (micro- or macro-) albuminuria. The low number of individuals with the minor homozygous genotype (i.e. AA) who had albuminuria may have posed a limitation for these analyses, and therefore the sensitivity of these findings is disputable. Therefore, further investigation in larger sample sizes is warranted to confirm these findings.

In conclusion, in two Dutch cohorts, both consisting of individuals with NGM, IGM, and T2DM, we found that the AA genotype of SNP rs1800624 (-374T/A) was protectively associated with blood pressure and arterial stiffness in individuals with NGM, but adversely associated with blood pressure and not with arterial stiffness in individuals with IGM or T2DM. These contrasting effects are currently unexplained, but suggest that metabolic abnormalities in response to hyperglycaemia influence the effects of RAGE polymorphisms, which needs further exploration. 


\section{REFERENCES}

1. Brownlee M. Biochemistry and molecular cell biology of diabetic complications. Nature. 2001;414(6865):813-820.

2. Goh SY, Cooper ME. The role of advanced glycation end products in progression and complications of diabetes. J Clin Endocrinol Metab. 2008;93(4):1143-1152.

3. Vlassara H. The AGE-receptor in the pathogenesis of diabetic complications. Diabetes Metab Res Rev. 2001;17(6):436-443.

4. Basta G. Receptor for advanced glycation endproducts and atherosclerosis: From basic mechanisms to clinical implications. Atherosclerosis. 2008;196(1):9-21.

5. Schmidt AM, Yan SD, Yan SF, Stern DM. The multiligand receptor RAGE as a progression factor amplifying immune and inflammatory responses. J Clin Invest. 2001;108(7):949-955.

6. Cipollone F, lezzi A, Fazia M, Zucchelli M, Pini B, Cuccurullo C, et al. The receptor RAGE as a progression factor amplifying arachidonate-dependent inflammatory and proteolytic response in human atherosclerotic plaques: role of glycemic control. Circulation. 2003;108(9):1070-1077.

7. Stern DM, Yan SD, Yan SF, Schmidt AM. Receptor for advanced glycation endproducts (RAGE) and the complications of diabetes. Ageing Res Rev. 2002;1(1):1-15.

8. Hofmann MA, Drury S, Hudson BI, Gleason MR, Qu W, Lu Y, et al. RAGE and arthritis: the G82S polymorphism amplifies the inflammatory response. Genes Immun. 2002;3(3):123-135.

9. Hudson BI, Stickland MH, Futers TS, Grant PJ. Effects of novel polymorphisms in the RAGE gene on transcriptional regulation and their association with diabetic retinopathy. Diabetes. 2001;50(6):1505-1511.

10. Mooy JM, Grootenhuis PA, de Vries H, Valkenburg HA, Bouter LM, Kostense PJ, et al. Prevalence and determinants of glucose intolerance in a Dutch caucasian population. The Hoorn Study. Diabetes Care. 1995;18(9):1270-1273.

11. Henry RM, Kostense PJ, Spijkerman AM, Dekker JM, Nijpels G, Heine RJ, et al. Arterial stiffness increases with deteriorating glucose tolerance status: the Hoorn Study. Circulation. 2003;107(16):2089-2095.

12. Kruijshoop M, Feskens EJ, Blaak EE, de Bruin TW. Validation of capillary glucose measurements to detect glucose intolerance or type 2 diabetes mellitus in the general population. Clin Chim Acta. 2004;341(1-2):3340.

13. Du H, van der AD, van Bakel MM, van der Kallen $C J$, Blaak EE, van Greevenbroek MM, et al. Glycemic index and glycemic load in relation to food and nutrient intake and metabolic risk factors in a Dutch population. Am J Clin Nutr. 2008;87(3):655-661.

14. Jacobs M, van Greevenbroek MM, van der Kallen CJ, Ferreira I, Blaak EE, Feskens EJ, et al. Low-grade inflammation can partly explain the association between the metabolic syndrome and either coronary artery disease or severity of peripheral arterial disease: the CODAM study. Eur J Clin Invest. 2009;39(6):437444.

15. Spijkerman AM, Adriaanse MC, Dekker JM, Nijpels G, Stehouwer CD, Bouter LM, et al. Diabetic patients detected by population-based stepwise screening already have a diabetic cardiovascular risk profile. Diabetes Care. 2002;25(10):1784-1789.

16. van Dam RM, Boer JM, Feskens EJ, Seidell JC. Parental history of diabetes modifies the association between abdominal adiposity and hyperglycemia. Diabetes Care. 2001;24(8):1454-1459.

17. Alberti KG, Zimmet PZ. Definition, diagnosis and classification of diabetes mellitus and its complications. Part 1: diagnosis and classification of diabetes mellitus provisional report of a WHO consultation. Diabet Med. 1998;15(7):539-553.

18. Gaens KH, van Der Kallen CJ, van Greevenbroek MM, Feskens EJ, Stehouwer CD, Schalkwijk CG. Receptor for advanced glycation end product polymorphisms and type 2 diabetes: the CODAM study. Ann N Y Acad Sci. 2008;1126:162-165.

19. Chalmers J, MacMahon S, Mancia G, Whitworth J, Beilin L, Hansson L, et al. 1999 World Health Organization-International Society of Hypertension Guidelines for the management of hypertension. Guidelines sub-committee of the World Health Organization. Clin Exp Hypertens. 1999;21(5-6):1009-1060. 
20. Laurent S, Cockcroft J, Van Bortel L, Boutouyrie P, Giannattasio C, Hayoz D, et al. Expert consensus document on arterial stiffness: methodological issues and clinical applications. Eur Heart $J$. 2006;27(21):2588-2605.

21. Levey AS, Bosch JP, Lewis JB, Greene T, Rogers N, Roth D. A more accurate method to estimate glomerular filtration rate from serum creatinine: a new prediction equation. Modification of Diet in Renal Disease Study Group. Ann Intern Med. 1999;130(6):461-470.

22. Lambers Heerspink HJ, Brinkman JW, Bakker SJ, Gansevoort RT, de Zeeuw D. Update on microalbuminuria as a biomarker in renal and cardiovascular disease. Curr Opin Nephrol Hypertens. 2006;15(6):631-636.

23. Kankova K, Stejskalova A, Hertlova M, Znojil V. Haplotype analysis of the RAGE gene: identification of a haplotype marker for diabetic nephropathy in type 2 diabetes mellitus. Nephrol Dial Transplant. 2005;20(6):1093-1102.

24. Yoon SJ, Park S, Shim CY, Park CM, Ko YG, Choi D, et al. Association of RAGE gene polymorphisms with coronary artery disease in the Korean population. Coron Artery Dis. 2007;18(1):1-8.

25. Zee RY, Romero JR, Gould JL, Ricupero DA, Ridker PM. Polymorphisms in the advanced glycosylation end product-specific receptor gene and risk of incident myocardial infarction or ischemic stroke. Stroke. 2006;37(7):1686-1690.

26. Kalousova M, Jachymova M, Mestek O, Hodkova M, Kazderova M, Tesar V, et al. Receptor for advanced glycation end products--soluble form and gene polymorphisms in chronic haemodialysis patients. Nephrol Dial Transplant. 2007;22(7):2020-2026.

27. Lindholm E, Bakhtadze E, Sjogren M, Cilio CM, Agardh E, Groop L, et al. The -374 T/A polymorphism in the gene encoding RAGE is associated with diabetic nephropathy and retinopathy in type 1 diabetic patients. Diabetologia. 2006;49(11):2745-2755.

28. Pettersson-Fernholm K, Forsblom C, Hudson BI, Perola M, Grant PJ, Groop PH. The functional -374 T/A RAGE gene polymorphism is associated with proteinuria and cardiovascular disease in type 1 diabetic patients. Diabetes. 2003;52(3):891-894.

29. Li J, Schmidt AM. Characterization and functional analysis of the promoter of RAGE, the receptor for advanced glycation end products. J Biol Chem. 1997;272(26):16498-16506.

30. Poirier O, Nicaud V, Vionnet N, Raoux S, Tarnow L, Vlassara H, et al. Polymorphism screening of four genes encoding advanced glycation end-product putative receptors. Association study with nephropathy in type 1 diabetic patients. Diabetes. 2001;50(5):1214-1218.

31. Shim CY, Park S, Yoon SJ, Park HY, Kim HT, Oh B, et al. Association of RAGE gene polymorphisms with instent restenosis in non-diabetic Korean population. Cardiology. 2007;107(4):261-268.

32. dos Santos KG, Canani LH, Gross JL, Tschiedel B, Pires Souto KE, Roisenberg I. The -374A allele of the receptor for advanced glycation end products gene is associated with a decreased risk of ischemic heart disease in African-Brazilians with type 2 diabetes. Mol Genet Metab. 2005;85(2):149-156.

33. Picheth G, Costantini CO, Pedrosa FO, Leme da Rocha Martinez T, Maltempi de Souza E. The -374A allele of the receptor for advanced glycation end products (RAGE) gene promoter is a protective factor against cardiovascular lesions in type 2 diabetes mellitus patients. Clin Chem Lab Med. 2007;45(10):1268-1272.

34. JiXiong X, BiLin X, MingGong Y, ShuQin L. -429T/C and -374T/A polymorphisms of RAGE gene promoter are not associated with diabetic retinopathy in Chinese patients with type 2 diabetes. Diabetes Care. 2003;26(9):2696-2697.

35. Falcone C, Geroldi D, Buzzi MP, Emanuele E, Yilmaz Y, Fontana JM, et al. The -374T/A RAGE polymorphism protects against future cardiac events in nondiabetic patients with coronary artery disease. Arch Med Res. 2008;39(3):320-325.

36. Falcone C, Campo I, Emanuele E, Buzzi MP, Geroldi D, Belvito C, et al. -374T/A polymorphism of the RAGE gene promoter in relation to severity of coronary atherosclerosis. Clin Chim Acta. 2005;354(1-2):111-116.

37. Lindholm E, Bakhtadze E, Cilio C, Agardh E, Groop L, Agardh CD. Association between LTA, TNF and AGER polymorphisms and late diabetic complications. PLOS ONE. 2008;3(6):e2546.

38. Ramprasad S, Radha V, Mathias RA, Majumder PP, Rao MR, Rema M. Rage gene promoter polymorphisms and diabetic retinopathy in a clinic-based population from South India. Eye. 2007;21(3):395-401.

39. Kirbis J, Milutinovic A, Steblovnik K, Teran N, Terzic R, Zorc M. The -429 T/C and -374 T/A gene polymorphisms of the receptor of advanced glycation end products gene (RAGE) are not risk factors for coronary artery disease in Slovene population with type 2 diabetes. Coll Antropol. 2004;28(2):611-616.

40. Globocnik Petrovic M, Steblovnik K, Peterlin B, Petrovic D. The - 429 T/C and - 374 T/A gene polymorphisms of the receptor of advanced glycation end products gene are not risk factors for diabetic retinopathy in Caucasians with type 2 diabetes. Klin Monatsbl Augenheilkd. 2003;220(12):873-876. 
41. Hofmann MA, Yang Q, Harja E, Kedia P, Gregersen PK, Cupples LA, et al. The RAGE Gly82Ser polymorphism is not associated with cardiovascular disease in the Framingham offspring study. Atherosclerosis. 2005;182(2):301-305.

42. Yoshioka K, Yoshida T, Takakura Y, Umekawa T, Kogure A, Toda H, et al. Association study of G1704T and G82S polymorphisms of RAGE gene for microalbuminuria in Japanese type 2 diabetic patients. Metabolism. 2005;54(4):488-491.

43. Yoshioka K, Yoshida T, Takakura Y, Umekawa T, Kogure A, Toda H, et al. Relation between polymorphisms G1704T and G82S of rage gene and diabetic retinopathy in Japanese type 2 diabetic patients. Intern Med. 2005;44(5):417-421.

44. Pulkkinen A, Viitanen L, Kareinen A, Lehto S, Laakso M. Gly82Ser polymorphism of the receptor of advanced glycation end product gene is not associated with coronary heart disease in Finnish nondiabetic subjects or in patients with type 2 diabetes. Diabetes Care. 2000;23(6):864.

45. Prevost G, Fajardy I, Besmond C, Balkau B, Tichet J, Fontaine P, et al. Polymorphisms of the receptor of advanced glycation endproducts (RAGE) and the development of nephropathy in type 1 diabetic patients. Diabetes Metab. 2005;31(1):35-39.

46. Kumaramanickavel G, Ramprasad VL, Sripriya S, Upadyay NK, Paul PG, Sharma T. Association of Gly82Ser polymorphism in the RAGE gene with diabetic retinopathy in type II diabetic Asian Indian patients. J Diabetes Complications. 2002;16(6):391-394.

47. Liu L, Xiang K. RAGE Gly82Ser polymorphism in diabetic microangiopathy. Diabetes Care. 1999;22(4):646.

48. Matsunaga-Irie S, Maruyama T, Yamamoto Y, Motohashi Y, Hirose H, Shimada A, et al. Relation between development of nephropathy and the p22phox C242T and receptor for advanced glycation end product G1704T gene polymorphisms in type 2 diabetic patients. Diabetes Care. 2004;27(2):303-307.

49. Kankova K, Beranek M, Hajek D, Vlkova E. Polymorphisms 1704G/T, 2184A/G, and 2245G/A in the rage gene are not associated with diabetic retinopathy in NIDDM: pilot study. Retina. 2002;22(1):119-121. 


\section{APPENDIX}

Table A7.1 Levels of blood pressure and arterial stiffness across the genotypes of the nine RAGE polymorphisms

\begin{tabular}{|c|c|c|c|c|c|c|c|c|c|c|c|c|c|}
\hline \multirow{2}{*}{ SNP } & \multirow{2}{*}{ genotype } & \multicolumn{2}{|r|}{$\mathrm{n}$} & \multicolumn{2}{|c|}{ SBP } & \multicolumn{2}{|c|}{ DBP } & \multicolumn{2}{|c|}{$\mathrm{PP}$} & \multicolumn{2}{|r|}{$\mathrm{n}$} & \multicolumn{2}{|c|}{ Total arterial stiffness score } \\
\hline & & NGM & IGM/T2DM & NGM & IGM/T2DM & NGM & IGM/T2DM & NGM & IGM/T2DM & NGM & IGM/T2DM & NGM & IGM/T2DM \\
\hline \multirow{3}{*}{ rs204995 } & $\mathrm{TT}$ & 315 & 396 & $135.6 \pm 1.0$ & $146.2 \pm 1.0$ & $79.9 \pm 0.5$ & $84.1 \pm 0.5$ & $55.7 \pm 0.8$ & $62.1 \pm 0.8$ & 127 & 205 & $-0.36 \pm 0.08$ & $0.20 \pm 0.06$ \\
\hline & $\mathrm{TC}$ & 195 & 238 & $138.9 \pm 1.3$ & $144.9 \pm 1.2$ & $81.6 \pm 0.7$ & $84.2 \pm 0.7$ & $57.3 \pm 1.0$ & $60.7 \pm 1.0$ & 76 & 119 & $-0.19 \pm 0.11$ & $0.20 \pm 0.08$ \\
\hline & $\mathrm{CC}$ & 25 & 40 & $139.5 \pm 3.5$ & $147.1 \pm 3.0$ & $83.4 \pm 1.9$ & $85.1 \pm 1.6$ & $56.1 \pm 2.7$ & $62.0 \pm 2.5$ & 11 & 23 & $-0.31 \pm 0.28$ & $0.12 \pm 0.19$ \\
\hline \multirow{3}{*}{$\begin{array}{l}\text { rs1800624 } \\
(-374 T / A)\end{array}$} & TT & 257 & 349 & $136.6 \pm 1.1$ & $145.1 \pm 1.0$ & $80.5 \pm 0.6$ & $84.4 \pm 0.5$ & $56.1 \pm 0.9$ & $60.7 \pm 0.8$ & 106 & 191 & $-0.21 \pm 0.09$ & $0.12 \pm 0.07$ \\
\hline & TA & 230 & 283 & $137.8 \pm 1.2$ & $145.4 \pm 1.1$ & $81.5 \pm 0.6$ & $83.9 \pm 0.6$ & $56.3 \pm 0.9$ & $61.5 \pm 0.9$ & 88 & 124 & $-0.39 \pm 0.10$ & $0.24 \pm 0.08$ \\
\hline & AA & 45 & 54 & $132.0 \pm 2.6$ & $151.4 \pm 2.5$ & $76.7 \pm 1.4$ & $86.2 \pm 1.4$ & $55.3 \pm 2.0$ & $65.2 \pm 2.1$ & 16 & 26 & $-0.85 \pm 0.23$ & $0.28 \pm 0.18$ \\
\hline \multirow{3}{*}{$\begin{array}{l}\text { rs1800625 } \\
(-429 T / C)\end{array}$} & $\mathrm{TT}$ & 344 & 460 & $135.5 \pm 1.0$ & $145.5 \pm 0.9$ & $80.0 \pm 0.5$ & $84.2 \pm 0.5$ & $55.4 \pm 0.7$ & $61.4 \pm 0.7$ & 137 & 228 & $-0.38 \pm 0.08$ & $0.20 \pm 0.06$ \\
\hline & $\mathrm{TC}$ & 179 & 208 & $139.1 \pm 1.3$ & $145.9 \pm 1.3$ & $81.5 \pm 0.7$ & $84.7 \pm 0.7$ & $57.6 \pm 1.0$ & $61.2 \pm 1.0$ & 67 & 102 & $-0.21 \pm 0.12$ & $0.24 \pm 0.09$ \\
\hline & $\mathrm{CC}$ & 14 & 24 & $136.8 \pm 4.7$ & $144.4 \pm 3.8$ & $82.6 \pm 2.6$ & $82.7 \pm 2.1$ & $54.2 \pm 3.7$ & $61.7 \pm 3.1$ & 9 & 17 & $-0.40 \pm 0.31$ & $-0.10 \pm 0.22$ \\
\hline \multirow{3}{*}{$\begin{array}{l}\text { rs2070600 } \\
\text { (Gly82Ser) }\end{array}$} & $\mathrm{CC}$ & 501 & 652 & $136.7 \pm 0.8$ & $145.5 \pm 0.7$ & $80.6 \pm 0.4$ & $84.2 \pm 0.4$ & $56.1 \pm 0.6$ & $61.2 \pm 0.6$ & 208 & 336 & $-0.31 \pm 0.06$ & $0.20 \pm 0.05$ \\
\hline & $\mathrm{CT}$ & 34 & 39 & $138.6 \pm 3.0$ & $148.2 \pm 3.0$ & $80.2 \pm 1.7$ & $83.4 \pm 1.6$ & $58.4 \pm 2.3$ & $64.9 \pm 2.5$ & 12 & 20 & $-0.22 \pm 0.27$ & $0.10 \pm 0.21$ \\
\hline & $\mathrm{TT}$ & 1 & 0 & $140.9 \pm 17.6$ & - & $81.7 \pm 9.7$ & - & $59.2 \pm 13.6$ & - & 0 & 0 & - & - \\
\hline \multirow{3}{*}{ rs1035798 } & $\mathrm{CC}$ & 259 & 354 & $136.5 \pm 1.1$ & $145.6 \pm 1.0$ & $80.6 \pm 0.6$ & $84.4 \pm 0.5$ & $56.0 \pm 0.8$ & $61.2 \pm 0.8$ & 108 & 198 & $-0.19 \pm 0.09$ & $0.14 \pm 0.07$ \\
\hline & $\mathrm{CT}$ & 231 & 277 & $137.3 \pm 1.2$ & $144.9 \pm 1.1$ & $81.3 \pm 0.6$ & $83.6 \pm 0.6$ & $56.0 \pm 0.9$ & $61.3 \pm 0.9$ & 92 & 126 & $-0.37 \pm 0.10$ & $0.26 \pm 0.08$ \\
\hline & $\mathrm{TT}$ & 43 & 51 & $134.2 \pm 2.7$ & $151.5 \pm 2.6$ & $76.5 \pm 1.5$ & $86.7 \pm 1.4$ & $57.7 \pm 2.1$ & $64.8 \pm 2.2$ & 16 & 26 & $-0.91 \pm 0.23$ & $0.87 \pm 0.18$ \\
\hline \multirow{3}{*}{$\begin{array}{l}\text { rs184003 } \\
(+1704 G / T)\end{array}$} & GG & 459 & 612 & $136.7 \pm 0.8$ & $145.4 \pm 0.8$ & $80.8 \pm 0.5$ & $84.3 \pm 0.4$ & $55.9 \pm 0.6$ & $61.1 \pm 0.6$ & 187 & 314 & $-0.36 \pm 0.07$ & $0.22 \pm 0.05$ \\
\hline & GT & 80 & 77 & $136.3 \pm 2.0$ & $147.1 \pm 2.2$ & $80.1 \pm 1.1$ & $84.1 \pm 1.1$ & $56.2 \pm 1.5$ & $63.0 \pm 1.8$ & 32 & 38 & $-0.08 \pm 0.16$ & $-0.07 \pm 0.15$ \\
\hline & TT & 4 & 4 & $132.3 \pm 8.9$ & $151.7 \pm 9.4$ & $73.8 \pm 4.8$ & $85.8 \pm 5.0$ & $58.5 \pm 6.8$ & $67.0 \pm 7.7$ & 1 & 3 & $1.17 \pm 0.93$ & $0.34 \pm 0.53$ \\
\hline
\end{tabular}


Table A7.1 (continued)

\begin{tabular}{|c|c|c|c|c|c|c|c|c|c|c|c|c|c|}
\hline \multirow{2}{*}{ SNP } & \multirow{2}{*}{ genotype } & \multicolumn{2}{|c|}{$n$} & \multicolumn{2}{|c|}{ SBP } & \multicolumn{2}{|c|}{ DBP } & \multicolumn{2}{|c|}{ PP } & \multirow{2}{*}{\multicolumn{2}{|c|}{$\frac{n}{\text { NGM IGM/T2DM }}$}} & \multicolumn{2}{|c|}{ Total arterial stiffness score } \\
\hline & & NGMI & $\mathrm{M} / \mathrm{T} 2 \mathrm{DM}$ & NGM & IGM/T2DM & NGM & IGM/T2DM & NGM & IGM/T2DM & & & NGM & IGM/T2DM \\
\hline \multirow{3}{*}{ rs3134943 } & GG & 425 & 519 & $136.7 \pm 0.9$ & $145.8 \pm 0.8$ & $80.7 \pm 0.5$ & $84.3 \pm 0.4$ & $56.0 \pm 0.6$ & $61.5 \pm 0.7$ & 177 & 262 & $-0.30 \pm 0.07$ & $0.19 \pm 0.06$ \\
\hline & GA & 110 & 166 & $136.6 \pm 1.7$ & $145.3 \pm 1.5$ & $79.9 \pm 0.9$ & $84.2 \pm 0.8$ & $56.7 \pm 1.3$ & $61.1 \pm 1.2$ & 42 & 90 & $-0.38 \pm 0.14$ & $0.20 \pm 0.10$ \\
\hline & AA & 6 & 11 & $127.7 \pm 7.2$ & $142.8 \pm 5.7$ & $81.6 \pm 4.0$ & $81.7 \pm 3.0$ & $46.0 \pm 5.5$ & $61.1 \pm 4.7$ & 2 & 3 & $-1.05 \pm 0.67$ & $0.07 \pm 0.53$ \\
\hline \multirow{3}{*}{ rs9469089 } & GG & 385 & 482 & $136.7 \pm 0.9$ & $146.0 \pm 0.9$ & $80.6 \pm 0.5$ & $84.3 \pm 0.5$ & $56.1 \pm 0.7$ & $61.7 \pm 0.7$ & 153 & 240 & $-0.37 \pm 0.07$ & $0.17 \pm 0.06$ \\
\hline & GC & 134 & 187 & $137.1 \pm 1.5$ & $144.4 \pm 1.4$ & $80.5 \pm 0.8$ & $84.3 \pm 0.7$ & $56.6 \pm 1.2$ & $60.1 \pm 1.1$ & 59 & 100 & $-0.25 \pm 0.12$ & $0.18 \pm 0.09$ \\
\hline & $\mathrm{CC}$ & 16 & 27 & $128.9 \pm 4.4$ & $148.0 \pm 3.7$ & $79.6 \pm 2.4$ & $84.2 \pm 1.9$ & $49.3 \pm 3.4$ & $63.8 \pm 3.0$ & 7 & 15 & $-0.18 \pm 0.35$ & $0.43 \pm 0.24$ \\
\hline \multirow{3}{*}{ rs3134945 } & GG & 321 & 425 & $136.0 \pm 1.0$ & $146.1 \pm 0.9$ & $80.2 \pm 0.5$ & $84.2 \pm 0.5$ & $55.7 \pm 0.8$ & $61.9 \pm 0.8$ & 133 & 215 & $-0.38 \pm 0.08$ & $0.22 \pm 0.06$ \\
\hline & GT & 193 & 233 & $137.8 \pm 1.3$ & $145.3 \pm 1.2$ & $81.0 \pm 0.7$ & $84.4 \pm 0.7$ & $56.9 \pm 1.0$ & $60.9 \pm 1.0$ & 75 & 117 & $-0.20 \pm 0.11$ & $0.21 \pm 0.09$ \\
\hline & TT & 20 & 29 & $139.0 \pm 4.0$ & $141.5 \pm 3.6$ & $81.7 \pm 2.2$ & $82.3 \pm 1.9$ & $57.3 \pm 3.1$ & $59.2 \pm 2.9$ & 9 & 21 & $-0.44 \pm 0.31$ & $-0.25 \pm 0.20$ \\
\hline
\end{tabular}

Data are expressed as mean \pm SE. NGM, normal glucose metabolism; IGM, impaired glucose metabolism; T2DM, type 2 diabetes mellitus; SBP, systolic blood pressure in mmHg; $D B P$, diastolic blood pressure in $\mathrm{mmHg}$; $P$, pulse pressure in $\mathrm{mmHg}$; ${ }^{a}$ data from the Hoorn study only. Significant associations are indicated in bold. All analyses were adjusted for age, sex, study population and use of blood pressure-, lipid- and glucose-lowering medication. 
Table A7.2 Prevalent CVD and markers of atherosclerosis across the genotypes of nine RAGE polymorphisms

\begin{tabular}{|c|c|c|c|c|c|c|c|c|c|}
\hline \multirow{2}{*}{ SNP } & \multirow{2}{*}{ genotype } & \multicolumn{2}{|r|}{$n$} & \multicolumn{2}{|c|}{ Prevalent CVD (\%) } & \multicolumn{2}{|c|}{ CIMT } & \multicolumn{2}{|c|}{$A B I$} \\
\hline & & $N G M$ & IGM/T2DM & $N G M$ & $I G M / T 2 D M$ & $N G M$ & IGM/T2DM & $N G M$ & IGM/T2DM \\
\hline \multirow{3}{*}{ rs204995 } & TT & 315 & 396 & 32.4 & 41.3 & $0.79 \pm 0.01$ & $0.84 \pm 0.01$ & $1.17 \pm 0.01$ & $1.15 \pm 0.01$ \\
\hline & $\mathrm{TC}$ & 195 & 238 & 27.0 & 42.6 & $0.80 \pm 0.01$ & $0.87 \pm 0.01$ & $1.17 \pm 0.01$ & $1.13 \pm 0.01$ \\
\hline & $\mathrm{CC}$ & 25 & 40 & 22.2 & 43.9 & $0.78 \pm 0.03$ & $0.82 \pm 0.03$ & $1.14 \pm 0.02$ & $1.16 \pm 0.03$ \\
\hline \multirow{3}{*}{$\begin{array}{l}\text { rs1800624 } \\
(-374 T / A)\end{array}$} & TT & 257 & 349 & 26.2 & 42.6 & $0.79 \pm 0.01$ & $0.85 \pm 0.01$ & $1.18 \pm 0.01$ & $1.14 \pm 0.01$ \\
\hline & TA & 230 & 283 & 31.0 & 42.6 & $0.79 \pm 0.01$ & $0.84 \pm 0.01$ & $1.17 \pm 0.01$ & $1.14 \pm 0.01$ \\
\hline & $A A$ & 45 & 54 & 36.2 & 42.5 & $0.80 \pm 0.02$ & $0.86 \pm 0.02$ & $1.16 \pm 0.02$ & $1.16 \pm 0.03$ \\
\hline \multirow{3}{*}{$\begin{array}{l}\text { rs1800625 } \\
(-429 T / C)\end{array}$} & $\mathrm{TT}$ & 344 & 460 & 30.2 & 42.2 & $0.79 \pm 0.01$ & $0.84 \pm 0.01$ & $1.17 \pm 0.01$ & $1.15 \pm 0.01$ \\
\hline & TC & 179 & 208 & 27.4 & 43.3 & $0.79 \pm 0.01$ & $0.88 \pm 0.01$ & $1.17 \pm 0.01$ & $1.14 \pm 0.01$ \\
\hline & $\mathrm{CC}$ & 14 & 24 & 24.8 & 44.5 & $0.79 \pm 0.04$ & $0.82 \pm 0.03$ & $1.14 \pm 0.03$ & $1.12 \pm 0.04$ \\
\hline \multirow{3}{*}{$\begin{array}{l}\text { rs2070600 } \\
\text { (Gly82Ser) }\end{array}$} & $\mathrm{CC}$ & 501 & 652 & 28.7 & 41.7 & $0.80 \pm 0.01$ & $0.85 \pm 0.01$ & $1.17 \pm 0.00$ & $1.15 \pm 0.01$ \\
\hline & $\mathrm{CT}$ & 34 & 39 & 41.1 & 42.6 & $0.78 \pm 0.03$ & $0.85 \pm 0.03$ & $1.20 \pm 0.02$ & $1.13 \pm 0.03$ \\
\hline & TT & 1 & 0 & 54.6 & 43.6 & $0.62 \pm 0.15$ & - & $1.11 \pm 0.11$ & - \\
\hline \multirow{3}{*}{ rs1035798 } & $\mathrm{CC}$ & 259 & 354 & 26.2 & 41.5 & $0.79 \pm 0.01$ & $0.85 \pm 0.01$ & $1.17 \pm 0.01$ & $1.14 \pm 0.01$ \\
\hline & CT & 231 & 277 & 31.4 & 42.6 & $0.80 \pm 0.01$ & $0.85 \pm 0.01$ & $1.17 \pm 0.01$ & $1.15 \pm 0.01$ \\
\hline & $\mathrm{TT}$ & 43 & 51 & 37.1 & 43.7 & $0.81 \pm 0.02$ & $0.87 \pm 0.02$ & $1.16 \pm 0.02$ & $1.15 \pm 0.03$ \\
\hline \multirow{3}{*}{$\begin{array}{l}\text { rs184003 } \\
(+1704 G / T)\end{array}$} & GG & 459 & 612 & 29.3 & 41.0 & $0.79 \pm 0.01$ & $0.85 \pm 0.01$ & $1.17 \pm 0.01$ & $1.15 \pm 0.01$ \\
\hline & GT & 80 & 77 & 31.4 & 47.5 & $0.79 \pm 0.02$ & $0.84 \pm 0.02$ & $1.18 \pm 0.01$ & $1.14 \pm 0.02$ \\
\hline & $\mathrm{TT}$ & 4 & 4 & 33.5 & 54.1 & $0.87 \pm 0.09$ & $0.84 \pm 0.08$ & $1.22 \pm 0.05$ & $1.15 \pm 0.09$ \\
\hline \multirow{3}{*}{ rs3134943 } & GG & 425 & 519 & 29.3 & 42.7 & $0.80 \pm 0.01$ & $0.85 \pm 0.01$ & $1.18 \pm 0.01$ & $1.15 \pm 0.01$ \\
\hline & $\mathrm{GA}$ & 110 & 166 & 31.4 & 41.3 & $0.79 \pm 0.01$ & $0.85 \pm 0.01$ & $1.16 \pm 0.01$ & $1.14 \pm 0.01$ \\
\hline & $A A$ & 6 & 11 & 33.6 & 40.0 & $0.81 \pm 0.06$ & $0.81 \pm 0.05$ & $1.17 \pm 0.04$ & $1.19 \pm 0.06$ \\
\hline \multirow{3}{*}{ rs9469089 } & GG & 385 & 482 & 32.3 & 42.9 & $0.80 \pm 0.01$ & $0.85 \pm 0.01$ & $1.17 \pm 0.01$ & $1.14 \pm 0.01$ \\
\hline & $\mathrm{GC}$ & 134 & 187 & 24.5 & 40.1 & $0.80 \pm 0.01$ & $0.85 \pm 0.01$ & $1.19 \pm 0.01$ & $1.16 \pm 0.01$ \\
\hline & $\mathrm{CC}$ & 16 & 27 & 18.1 & 37.4 & $0.72 \pm 0.04$ & $0.84 \pm 0.03$ & $1.17 \pm 0.03$ & $1.20 \pm 0.04$ \\
\hline \multirow{3}{*}{ rs3134945 } & GG & 321 & 425 & 29.9 & 42.9 & $0.80 \pm 0.01$ & $0.84 \pm 0.01$ & $1.17 \pm 0.01$ & $1.15 \pm 0.01$ \\
\hline & GT & 193 & 233 & 29.2 & 41.0 & $0.79 \pm 0.01$ & $0.87 \pm 0.01$ & $1.17 \pm 0.01$ & $1.13 \pm 0.01$ \\
\hline & TT & 20 & 29 & 28.5 & 39.1 & $0.80 \pm 0.04$ & $0.79 \pm 0.03$ & $1.17 \pm 0.02$ & $1.14 \pm 0.04$ \\
\hline
\end{tabular}

Data are expressed as mean \pm SE or percentages. NGM, normal glucose metabolism; IGM, impaired glucose metabolism; T2DM, type 2 diabetes mellitus; CVD, cardiovascular disease; CIMT, carotid intima-media thickness in mm; $\mathrm{ABI}$, ankle-brachial index. Significant associations are indicated in bold. All analyses were adjusted for age, sex, study population and use of blood pressure-, lipid- and glucose-lowering medication. 
Table A7.3 Markers of renal disease across the genotypes of the nine RAGE polymorphisms

\begin{tabular}{|c|c|c|c|c|c|c|c|}
\hline \multirow{2}{*}{ SNP } & \multirow{2}{*}{ genotype } & \multicolumn{2}{|r|}{$n$} & \multicolumn{2}{|c|}{ Albuminuria (\%) } & \multicolumn{2}{|c|}{ eGFR } \\
\hline & & $N G M$ & IGM/T2DM & $N G M$ & IGM/T2DM & $N G M$ & IGM/T2DM \\
\hline \multirow{3}{*}{ rs204995 } & TT & 312 & 396 & 6.9 & 9.5 & $71.1 \pm 0.7$ & $68.7 \pm 0.7$ \\
\hline & $\mathrm{TC}$ & 194 & 237 & 7.1 & 13.1 & $71.8 \pm 0.9$ & $67.9 \pm 0.9$ \\
\hline & $\mathrm{CC}$ & 25 & 40 & 7.2 & 15.5 & $68.9 \pm 2.4$ & $69.8 \pm 2.1$ \\
\hline \multirow{3}{*}{$\begin{array}{l}\text { rs1800624 } \\
(-374 T / A)\end{array}$} & TT & 256 & 349 & 5.8 & 11.0 & $72.3 \pm 0.7$ & $69.1 \pm 0.7$ \\
\hline & TA & 229 & 282 & 7.8 & 11.6 & $70.5 \pm 0.8$ & $69.2 \pm 0.8$ \\
\hline & AA & 44 & 54 & 10.4 & 12.3 & $72.5 \pm 1.8$ & $67.2 \pm 1.8$ \\
\hline \multirow{3}{*}{$\begin{array}{l}\text { rs1800625 } \\
(-429 T / C)\end{array}$} & $\mathrm{TT}$ & 342 & 459 & 6.8 & 11.4 & $71.4 \pm 0.6$ & $69.3 \pm 0.6$ \\
\hline & $\mathrm{TC}$ & 178 & 208 & 7.2 & 11.2 & $71.6 \pm 0.9$ & $67.9 \pm 0.9$ \\
\hline & $\mathrm{CC}$ & 14 & 24 & 7.6 & 11.1 & $68.6 \pm 3.2$ & $68.5 \pm 2.7$ \\
\hline \multirow{3}{*}{$\begin{array}{l}\text { rs2070600 } \\
\text { (Gly82Ser) }\end{array}$} & $\mathrm{CC}$ & 497 & 651 & 6.8 & 11.7 & $70.9 \pm 0.5$ & $68.1 \pm 0.5$ \\
\hline & $\mathrm{CT}$ & 34 & 39 & 10.4 & 5.0 & $72.8 \pm 2.0$ & $73.4 \pm 2.1$ \\
\hline & TT & 1 & 0 & 15.5 & 2.1 & $82.6 \pm 11.8$ & - \\
\hline \multirow{3}{*}{ rs1035798 } & $\mathrm{CC}$ & 258 & 354 & 5.3 & 10.7 & $72.3 \pm 0.7$ & $68.9 \pm 0.7$ \\
\hline & $\mathrm{CT}$ & 229 & 276 & 7.3 & 12.4 & $70.3 \pm 0.8$ & $68.2 \pm 0.8$ \\
\hline & TT & 42 & 51 & 10.1 & 14.2 & $70.3 \pm 1.8$ & $66.3 \pm 1.8$ \\
\hline \multirow{3}{*}{$\begin{array}{l}\text { rs184003 } \\
(+1704 \mathrm{G} / \mathrm{T})\end{array}$} & GG & 455 & 611 & 7.5 & 12.0 & $71.0 \pm 0.6$ & $68.5 \pm 0.5$ \\
\hline & GT & 80 & 77 & 4.1 & 8.5 & $72.4 \pm 1.3$ & $67.6 \pm 1.5$ \\
\hline & TT & 4 & 4 & 2.2 & 5.9 & $72.3 \pm 6.0$ & $61.2 \pm 6.6$ \\
\hline \multirow{3}{*}{ rs3134943 } & GG & 421 & 517 & 7.6 & 12.0 & $71.0 \pm 0.6$ & $68.1 \pm 0.6$ \\
\hline & $\mathrm{GA}$ & 110 & 167 & 4.7 & 10.6 & $71.6 \pm 1.1$ & $69.6 \pm 1.0$ \\
\hline & AA & 6 & 11 & 2.8 & 9.3 & $77.1 \pm 4.9$ & $73.1 \pm 3.9$ \\
\hline \multirow{3}{*}{ rs9469089 } & GG & 384 & 481 & 8.1 & 11.1 & $71.4 \pm 0.6$ & $68.2 \pm 0.6$ \\
\hline & $\mathrm{GC}$ & 131 & 187 & 3.8 & 10.8 & $69.6 \pm 1.0$ & $68.3 \pm 1.0$ \\
\hline & $\mathrm{CC}$ & 16 & 27 & 1.8 & 10.6 & $72.6 \pm 3.0$ & $72.9 \pm 2.6$ \\
\hline \multirow{3}{*}{ rs3134945 } & GG & 318 & 424 & 6.2 & 12.2 & $71.1 \pm 0.7$ & $68.6 \pm 0.6$ \\
\hline & GT & 192 & 233 & 7.2 & 11.0 & $70.9 \pm 0.9$ & $68.3 \pm 0.9$ \\
\hline & TT & 20 & 29 & 8.3 & 9.9 & $69.1 \pm 2.7$ & $65.4 \pm 2.5$ \\
\hline
\end{tabular}

Data are expressed as mean \pm SE. NGM, normal glucose metabolism; IGM, impaired glucose metabolism; T2DM, type 2 diabetes mellitus; Albuminuria, micro- or macroalbuminuria (\%); eGFR, estimated glomerular filtration rate. Significant associations are indicated in bold. All analyses were adjusted for age, sex, study population and use of blood pressure-, lipid- and glucose-lowering medication. 



\section{CHAPTER 8}

PLASMA LEVELS OF ADVANCED ŞLYCATION EMDPRODUCTS ARE NOT INDEPEKDENTLY ASSOCIATED WITH CARDIOVASCULAR DISEASE IN INDIVIDUALS WITH OR WITHOUT TYPE 2 DIABETES:

\section{THE HOORN AND CODAM STUDIES}

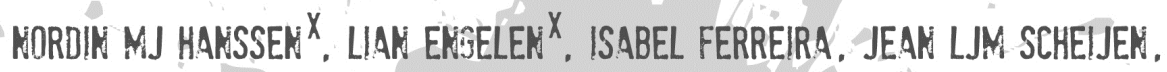
MAYA S HUIJBERTS, MARLEEK MJ VAN SGREEYENBROEX, CARLA JH YAN DER KALLEN. JACQUELINE M DEXKER, GIEL NIJPELS, COEM DA STEHOU'MER, CASPER S SCHALKW'IJK

${ }^{x}$ EQUAL CONTRIBUTION 


\section{ABSTRACT}

\section{Background}

Advanced glycation endproducts (AGEs) may contribute to cardiovascular disease (CVD), particularly among individuals with type 2 diabetes (T2DM). However, the epidemiological evidence of an adverse association between AGEs and (subclinical markers of) CVD remains uncertain. We therefore investigated, in a large sample of participants with various degrees of glucose metabolism, the associations of plasma AGEs with prevalent CVD, carotid intima-media thickness (CIMT) and ankle-brachial index (ABI).

\section{Methods}

We measured plasma levels of protein-bound $\mathrm{CML}, \mathrm{CEL}$ and pentosidine, and free $\mathrm{CML}$, CEL and MG-H1, in participants from two Dutch cohort studies of individuals ( $n=1,291$, $65 \pm 8$ years, $45 \%$ women) with normal glucose metabolism (NGM, $n=573$ ), impaired glucose metabolism (IGM, $n=304)$, or T2DM ( $n=414)$. Data were analyzed with multiple logistic or linear regression analyses.

\section{Results}

Protein-bound levels of CEL [32 (IQR: 25-40 vs 28 (22-35) nmol/mmol lysine] and pentosidine $[0.53(0.43-0.67)$ vs. $0.48(0.40-0.59) \mathrm{nmol} / \mathrm{mmol}$ lysine] as well as of free CEL [48 (39-62) vs. 45 (36-56) nmol/l] and MG-H1 [141 (96-209) vs. 116 (84-165) nmol/l] were higher in individuals with vs. without CVD, whereas protein-bound CML was lower [33 (2738 vs. 34 (29-39) $\mathrm{nmol} / \mathrm{mmol}$ lysine)]. However, these differences disappeared after adjustment for age, sex, cohort and other risk factors. Plasma AGEs were also not independently associated with $\mathrm{CIMT}$ or $\mathrm{ABI}$, except for a positive association between free $C M L$ and $A B I$ [standardized $\beta=0.106$ (95\% $\mathrm{Cl}: 0.064 ; 0.148)$ ].

\section{Conclusion}

Although experimental studies support the involvement of AGEs in diabetes-related CVD, we found no independent adverse associations of plasma AGEs with CVD, CIMT or ABI in a large sample of individuals with NGM, IGM and T2DM. 


\section{INTRODUCTION}

Evidence from both histological ${ }^{1-3}$ and experimental ${ }^{4-6}$ studies links advanced glycation endproducts (AGEs), a heterogeneous family of extensively sugar-modified proteins, to cardiovascular disease (CVD) and other vascular complications in diabetes. ${ }^{7} 8$ However, the epidemiological evidence of an adverse association between increased levels of AGEs and CVD or subclinical markers thereof, in individuals with ${ }^{9-13}$ or without ${ }^{12-18}$ type 2 diabetes (T2DM), remains uncertain, as studies thus far have yielded inconsistent findings.

Specifically, plasma levels of AGEs, such as $N^{\varepsilon}$-(carboxymethyl)lysine $(C M L), N^{\varepsilon}$ (carboxyethyl)lysine (CEL), pentosidine or 5-hydro-5-methylimidazolone (MG-H1), were cross-sectionally associated with (surrogate markers of) CVD $910,14,15$ and with incident $\mathrm{CVD}^{16,17}$ and all-cause mortality, ${ }^{17}$ whereas other studies showed no such associations ${ }^{11,12}$ ${ }^{18}$ or showed these in sub-populations only. ${ }^{13}$ These studies were often rather small

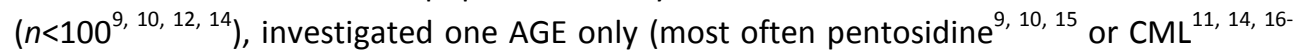
$\left.{ }^{18}\right)$, and/or have not taken potential confounding factors such as age ${ }^{10,14,18}$ and/or glomerular filtration rate ${ }^{10,12,14,18}$ (fully) into consideration. Furthermore, the vast majority determined plasma AGE levels with the use of immunoassays, ${ }^{10-18}$ which yield only semi-quantitative data and therefore should be interpreted with caution. Currently, specific analytical techniques, such as $\mathrm{HPLC}^{19}$ and (ultra-performance) liquid chromatography tandem MS (UPLC-MS/MS) ${ }^{20,21}$ offer a more accurate approach for the quantitative determination of specific AGE epitopes. Lastly, these studies have measured plasma protein-bound AGEs only. Although protein-bound AGEs encompass the largest fraction of the total plasma AGE pool, plasma AGEs are cleared from the circulation in the free form and these free AGEs might better reflect pathophysiological actions of glycation in tissues, ${ }^{22}$ but these have not been investigated in a large cohort study of individuals with and/or without T2DM.

In view of these considerations, we have investigated, in a large combined sample of participants from two cohort studies: firstly, the extent to which plasma levels of proteinbound CML, CEL and pentosidine measured with UPLC-MS/MS or HPLC differed across different levels of impaired glucose metabolism [i.e. normal (NGM), impaired (IGM) and T2DM], and secondly, whether these AGEs were associated with prevalent CVD and subclinical markers of atherosclerosis [i.e. carotid intima-media thickness (CIMT) and ankle-brachial index $(A B I)]$. Finally, similar analyses were conducted for the free levels of $\mathrm{CML}, \mathrm{CEL}$ and MG-H1 in the subpopulation in which these were available for assessment by means of UPLC-MS/MS. 


\section{METHODS}

\section{STUDY POPULATION}

The present study included participants from the baseline examination of the Cohort on Diabetes and Atherosclerosis Maastricht (CODAM) Study ${ }^{23}{ }^{24}$ and from the follow-up examination of the Hoorn Study, ${ }^{25}$ which were (by design) combined as described previously. ${ }^{26}$ Briefly, the CODAM study is an ongoing prospective cohort study that was designed to investigate the effects of obesity, glucose and lipid metabolism, lifestyle and genetic factors on CVD. Between 1999 and 2001 individuals were selected from a large population-based cohort on the basis of an elevated risk for T2DM and/or CVD. Individuals were included if they had Caucasian ethnicity, were older than 40 years and fulfilled one or more of the following criteria: body mass index (BMI) $>25 \mathrm{~kg} / \mathrm{m}^{2}$, positive family history for diabetes, history of gestational diabetes, postprandial glucose $>6.0 \mathrm{mmol} / \mathrm{L}$, glucosuria, or use of blood pressure (BP)-lowering medication. In total, 574 individuals were included and extensively characterized with regard to their metabolic, cardiovascular and lifestyle risk profiles during two visits to the University's research unit. ${ }^{23,24}$ The Hoorn study started in 1989 as a population-based cohort study investigating glucose metabolism status and CVD risk factors among a sample of the general population of Hoorn, the Netherlands. In 2000-2001, a follow-up examination was conducted in a selected group of 822 individuals consisting of a random sample of individuals with NGM and IGM and all individuals with T2DM of the survival cohort and 188 additional individuals with T2DM from the Hoorn Screening study. ${ }^{25,27}$

Individuals who had missing data on plasma AGEs $(n=88)$ or CVD status $(n=17)$ were excluded from the present analyses, resulting in a total study population of 1,291 individuals (554 from CODAM and 737 from Hoorn). Individuals excluded from analyses more often had T2DM and had on average somewhat higher CIMT, BMI, waist circumference, BP and lower HDL-cholesterol levels (data not shown). In both cohorts participants' glucose metabolism status was ascertained on the basis of fasting and 2-hour post-load glucose levels according to the WHO's criteria of $1999^{28}$ and as described in detailed elsewhere. ${ }^{24,25}$ In total, 573 individuals had NGM, 304 had IGM (including impaired fasting glucose and/or impaired glucose tolerance) and 414 had T2DM.

Written informed consent was obtained from all participants and both cohort studies were approved by the local Medical Ethics Committees (of the Maastricht University Medical Centre for the CODAM study and of the VU University Medical Center, Amsterdam, for the Hoorn study). 


\section{MEASUREMENT OF PLASMA ADVANCED GLYCATION ENDPRODUCTS}

Plasma AGEs were measured in EDTA plasma that was obtained from fasting venous blood samples that were stored at $-80^{\circ} \mathrm{C}$ until analysis.

Protein-bound AGEs. For the measurement of protein-bound CML, CEL and lysine, 25 $\mu \mathrm{L}$ plasma was mixed with $50 \mu \mathrm{L}$ water. After addition of $200 \mu \mathrm{L}$ sodium borohydride (100 $\mathrm{mmol} / \mathrm{L}$ ) dissolved in borate buffer ( $\mathrm{pH} \mathrm{9.2,} 200 \mathrm{mmol} / \mathrm{L})$, the samples were incubated at room temperature for 2 hours. Next, samples were deproteinized with $1000 \mu \mathrm{L}$ cold $\left(4^{\circ} \mathrm{C}\right)$ trifluoroacetic acid. After centrifugation $\left(4300 \mathrm{~g}, 4^{\circ} \mathrm{C}, 20 \mathrm{~min}\right)$ the supernatant was carefully removed. Samples were then hydrolyzed by adding $500 \mu \mathrm{L} 6 \mathrm{~N} \mathrm{HCl}$ and incubated for 18 hours at $110^{\circ} \mathrm{C}$. After hydrolysis, $40 \mu \mathrm{L}$ hydrolysate and $20 \mu \mathrm{L}$ internal standard (containing $1432 \mathrm{nmol} / \mathrm{L}\left[{ }^{2} \mathrm{H}_{2}\right]-\mathrm{CML}$ and $1378 \mathrm{nmol} / \mathrm{L}\left[{ }^{2} \mathrm{H}_{4}\right]-\mathrm{CEL}$ (Polypeptide Laboratories, San Diego, CA, USA) was mixed in a reaction vial. For CML and CEL, this mixture was evaporated to dryness under a stream of nitrogen gas at $70^{\circ} \mathrm{C}$ and subsequently derivatized with $100 \mu \mathrm{L} 1$-butanol: $\mathrm{HCl}(3: 1, v / v)$ for 90 minutes at $70^{\circ} \mathrm{C}$. Samples were then evaporated to dryness under nitrogen and redissolved in $200 \mu \mathrm{l}$ water. For lysine, $10 \mu \mathrm{L}$ hydrolysate was diluted in $800 \mu \mathrm{L}$ water. Twenty $\mu \mathrm{L}$ of this mixture and $20 \mu \mathrm{L}$ internal standard $\left[{ }^{13} \mathrm{C}_{6}\right]$ - L-Lysine $(40.16 \mu \mathrm{mol} / \mathrm{L}$, Cambridge Isotope Laboratories, Andover, MA, USA) was diluted in $500 \mu \mathrm{L} 10 \mathrm{mmol} / \mathrm{L}$ ammonia. Derivatized CML and CEL, and underivatized lysine were analyzed by UPLC (Acquity UPLC, Waters, Milford, USA) and detected in ESI positive multiple reaction monitoring (MRM) mode using a Xevo TQ MS (Waters, Milford, USA). Derivatives were separated on a reversed-phase C18 column (Acquity UPLC BEH C18, $50 \times 2.1 \mathrm{~mm}, 1.7 \mu \mathrm{m}$ ) with a linear gradient of $5 \mathrm{mmol} / \mathrm{L}$ ammonia and acetonitril at $48^{\circ} \mathrm{C}$ for $\mathrm{CML}$ and $\mathrm{CEL}$, and a linear gradient of $20 \mathrm{mmol} / \mathrm{L}$ ammonium formate and acetonitril at $30^{\circ} \mathrm{C}$ for lysine. The flow rate was $800 \mu \mathrm{L} / \mathrm{min}$ and the injection volume was $2 \mu \mathrm{L}$. Quantification of CML, CEL and lysine was performed by calculating the peak area ratio of each unlabeled peak area to the corresponding internal standard peak area. The MRM transitions for CML, CEL and lysine were 317.1>186.1, 331.1>186.1 and 147.2>84.2, respectively. The MRM transitions for the internal standards $\left[{ }^{2} \mathrm{H}_{2}\right]-\mathrm{CML},\left[{ }^{2} \mathrm{H}_{4}\right]-$ CEL and $\left[{ }^{13} C_{6}\right]$-L-Lysine were 319.1>186.1, 335.1>190.1 and 153.2>89.2, respectively. Electrospray ionization was done at a capillary voltage of $0.5 \mathrm{kV}$, a source temperature of $150^{\circ} \mathrm{C}$ and a desolvation temperature of $600^{\circ} \mathrm{C}$. For validation purposes, linearity was determined by adding standard solution of CML, CEL and lysine to water and plasma. A sixpoint calibration curve was prepared for CML (0-5250 nmol/L), CEL (0-6250 nmol/L) and lysine $(0-198.2 \mu \mathrm{mol} / \mathrm{L})$. The peak area ratio of $\mathrm{CML}$ and $\mathrm{CEL}$ multiplied by the concentration of each corresponding internal standard were plotted as a function of the concentration. The calibration curves for CML, CEL and lysine were linear over the described concentration ranges $\left(r^{2}>0.99\right)$ in both water and plasma. Mean slopes 
(response factors) for CML, CEL and lysine were 1.064 (CV, 8.9\%), 1.429 (CV, 6.0\%) and 0.994 (CV, 4.2\%), respectively. Intra- and inter-assay coefficients of variation were 4.8 and 7.0\% for CML, 5.0 and $9.7 \%$ for CEL, and 2.7 and $4.9 \%$ for lysine, respectively. The lower limits of quantification ( $\mathrm{s} / \mathrm{N}=10$ ) on column were $4.0 \mathrm{fmol}$ both for $\mathrm{CML}$ and $\mathrm{CEL}$ and 11 fmol for lysine, corresponding to a concentration of $200 \mathrm{nmol} / \mathrm{L}$ for CML and CEL and 254 $\mu \mathrm{mol} / \mathrm{L}$ for lysine. Method correlation of the described lysine analysis was tested in 44 plasma samples as compared with a bicinchoninic acid (BCA) protein assay. The described lysine analysis gave good correlation with the protein analysis $\left(r^{2}=0.930\right)$.

Pentosidine residues in plasma protein were measured using HPLC with a fluorescent detector, as described in detail elsewhere. ${ }^{19}$ Intra- and inter-assay CVs for pentosidine were $6.5 \%$ and $3.1 \%$, respectively.

Concentrations of CML, CEL and pentosidine residues in plasma protein were adjusted for levels of lysine and thus expressed as $\mathrm{nmol} / \mathrm{mmol}$ lysine.

Free AGEs. These measurements were confined to the CODAM Study population only, because plasma samples from the Hoorn study were unavailable for this purpose. In short, to $25 \mu \mathrm{L}$ internal standard, $50 \mu \mathrm{L}$ plasma was added. Samples were mixed thoroughly and subsequently deproteinized with $600 \mu \mathrm{L}$ of a mixture of methanol and acetonitrile $(1: 3$, by volume) and centrifuged at $14000 \mathrm{rpm}$ for $20 \mathrm{~min}$ at room temperature. The supernatant was transferred to a reaction vial and evaporated to dryness under a gentle stream of nitrogen at a temperature of $70^{\circ} \mathrm{C}$. Samples were then derivatized with $100 \mu \mathrm{L}$ 1-butanol: $\mathrm{HCl}(3: 1, \mathrm{v} / \mathrm{v})$ for 90 minutes at $70^{\circ} \mathrm{C}$. Next, the mixture was evaporated to dryness under a gentle stream of nitrogen at $70^{\circ} \mathrm{C}$. The derivatized residue was dissolved in $150 \mu \mathrm{L}$ MS water, and analyzed. Mean slopes (response factors) for CML, CEL and MG-H1 were 1.050 (CV, 10.1\%), 1.460 (CV, 3.2\%) and 1.010 (CV, 3.9\%), respectively. Intra- and inter-assay CVs were 2.8 and $7.1 \%$ for $\mathrm{CML}, 3.7$ and $6.4 \%$ for $\mathrm{CEL}$, and 3.7 and $5.1 \%$ for MG-H1, respectively.

\section{DEFINITION OF CARDIOVASCULAR DISEASE}

Prevalent CVD was defined as history of myocardial infarction, stroke or transient ischemic attack, non-traumatic limb amputation, coronary or peripheral artery bypass, angioplasty (self-reported), and/or signs of infarction or ischemia measured on a 12-lead ECG (Minnesota codes 1-1, 1-2, 1-3, 4-1, 4-2, 4-3, 5-1, 5-2, 5-3 or 7-1) or an $\mathrm{ABI}<0.9$ in either leg. $^{24,25}$ 


\section{CAROTID INTIMA-MEDIA THICKNESS AND ANKLE-BRACHIAL INDEX}

Common CIMT was measured by means of non-invasive ultrasonography according to guidelines for user procedures and with the use of valid methods and devices, as described previously. ${ }^{25}$ Briefly, common carotid arteries (left and right in the CODAM study, and right only in the Hoorn study) were visualized with an ultrasound scanner (Ultramark 4+, Advance Technology Laboratories, Bothel, Washington, USA in the CODAM study and Pie 350 Series, Pie Medical currently ESAOTE, Maastricht, the Netherlands in the Hoorn study) equipped with a 7.5. $\mathrm{MHz}$ linear array probe and connected to a personal computer. This computer was equipped with an acquisition system and a vessel wall movement detector software system based on radio frequency signal processing (Wall Track System 2, Pie Medical currently ESAOTE, Maastricht, the Netherlands). ${ }^{29}$ The CIMT was computed, by calculating the mean of all readings, these being at least 2 , as the distance between the leading edge interface between the lumen and the intima layer to the leading edge interface between the media and the adventitia layers at level of the posterior carotid artery wall. ${ }^{29,30} \mathrm{CIMT}$ data were available in 1,182 individuals.

In the CODAM study, systolic BP (SBP) was measured with a standard Doppler device (Mini Dopplex D900, Huntleigh Diagnostics Ltd, currently: E-Medical, Harmelen, the Netherlands) in the brachial arteries and twice in both the tibialis posterior and dorsalis pedis arteries of the lower extremities. ${ }^{24}$ In the Hoorn study, Doppler-assisted SBP measurements were performed in the brachial and posterior tibial arteries (Medasonics Vasculab, Moutainview, CA, USA). However, whenever ABI at the level of the posterior tibial artery was $<0.90$, or the Doppler flow signal was not audible, the ankle pressure was also measured at the dorsalis pedis or the peroneal arteries. In both studies, ABI was calculated for each leg by dividing the highest ankle pressure by the highest brachial pressure and the lowest $A B I$ of either leg was used in the analyses. ${ }^{31}$ Individuals with an $A B I>1.4(n=7)$, which is indicative of arterial calcification, were excluded from the analysis and $A B I$ data were thus available in 1,122 individuals.

\section{COVARIATES}

Study covariates included waist circumference, smoking status, mean arterial pressure (MAP; calculated as $2 * \mathrm{DBP}+\mathrm{SBP}) / 3$ ), total, $\mathrm{HDL}$ and $\mathrm{LDL}$ cholesterol, triglycerides, estimated (e) glomerular filtration rate (GFR; using the short Modification of Diet in Renal Disease equation ${ }^{32}$ ), presence of micro- or macroalbuminuria and use of BP-, lipid- and glucose-lowering medication, and were determined as described earlier. ${ }^{23,27,33}$ 


\section{STATISTICAL ANALYSES}

All statistical analyses were carried out using the Predictive Analytics SoftWare, version 18.0 (SPSS IBM Corporation, Armonk, NY, USA). Variables with a skewed distribution (i.e. $\mathrm{CML}, \mathrm{CEL}$, pentosidine, MG-H1, triglycerides) were $\log _{e}$ transformed prior to all analyses.

Imputation of missing covariate data. A total of 88 individuals ( $7 \%$ of the total population) had missing values for one $(n=82)$ or more $(n=6)$ of the covariates. The percentage of missing values per variable varied from $0.1 \%$ (current smoking) to $3.5 \%$ (BP). We used multiple imputation chained equations to impute those values rather than perform complete case analyses in order to decrease selection bias and increase the power of the analyses. ${ }^{34,35}$ With this procedure, the imputation model of a single missing variable used the information from all the other variables considered (listed in Table 1) as predictors by appropriate regression models (i.e. linear or logistic if variable to be imputed was continuous or dichotomous, respectively). We generated five imputed datasets, accounting for design features such as clustering within glucose metabolism status and stratification by cohort, which were used to fit the regression models of interest. Results reported were those retrieved from pooled analyses on all five imputed datasets.

Data analyses. General characteristics of the combined CODAM and Hoorn study populations were compared between individuals with and without prior CVD and across levels of glucose metabolism with the use of ANOVA or $\chi^{2}$-tests, for continuous or categorical data, respectively.

We used linear regression analyses to compare the levels of AGEs between individuals with IGM or T2DM vs. NGM (reference group), first, after adjustments for age, sex, and cohort (model 1) and additionally for waist circumference, smoking status, MAP, blood lipids, eGFR, albuminuria and use of medication (model 2). We used logistic regression analyses to investigate the associations between plasma AGEs and prevalent CVD and linear regression analyses to investigate the associations of plasma AGEs with $\mathrm{CIMT}$ and $\mathrm{ABI}$. These analyses were conducted in the whole study population and also stratified according to individuals' glucose metabolism status, and included adjustments for covariates as described in models 1 and 2 above. Results of these analyses are expressed in standardized regression coefficients to allow comparison of the strength of the associations between the different AGEs and outcome variables.

We also tested whether associations differed between cohorts or between men and women by adding interaction terms to our models, but did not find any such consistent interactions ( $p$-values for interaction were $>0.1$ ). Therefore, all results are shown without stratification for these factors. 
Table 8.1 General characteristics of the study population stratified according to prior CVD or glucose metabolism status

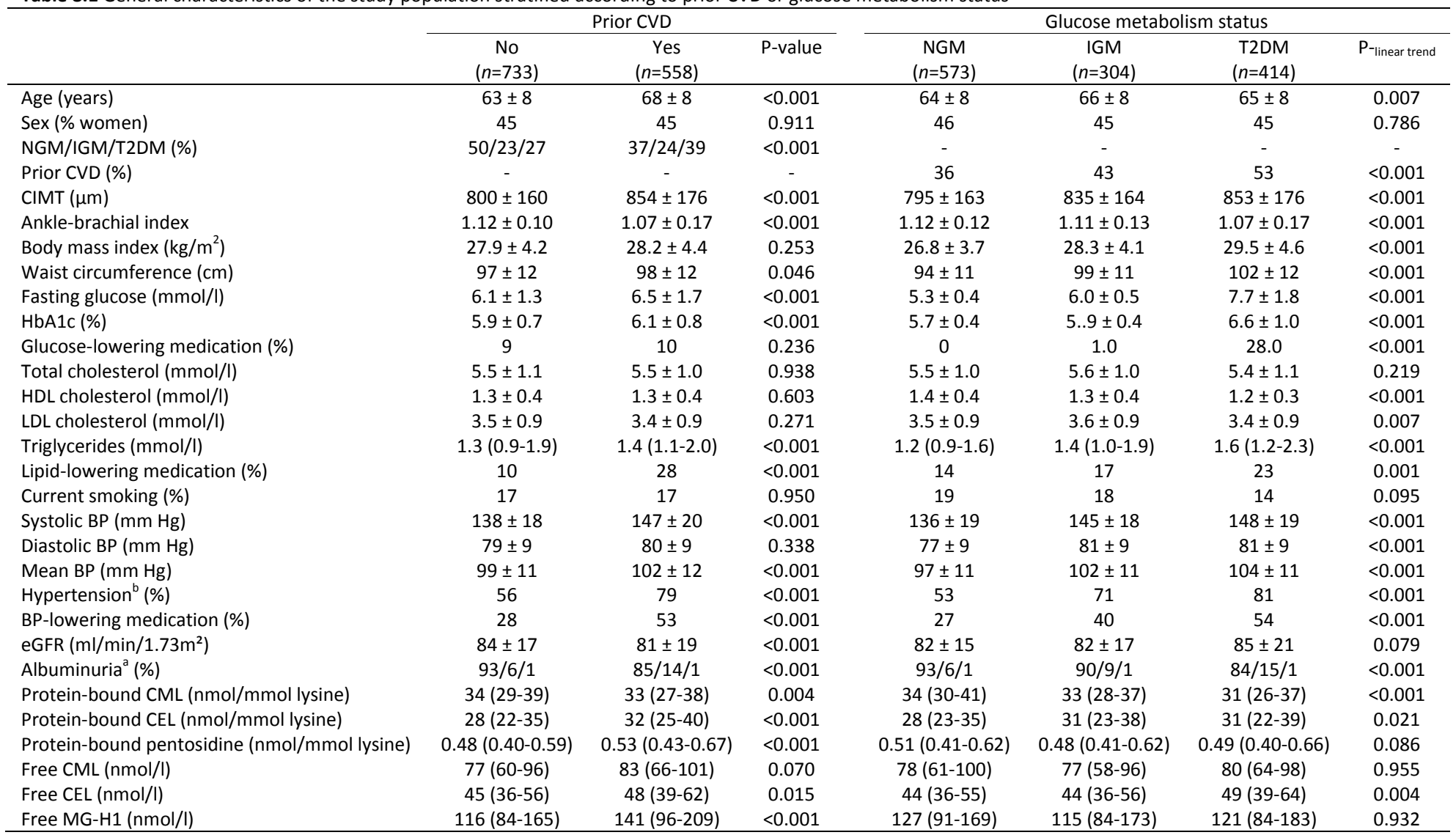

Free MG-H1 (nmol/l)

$116(84-165) \quad 141(96-209)-<0.001$

$127(91-169)$

$115(84-173)$

$121(84-183)$

Data are presented as means \pm SD, medians (IQR) or percentages, as appropriate. CVD, cardiovascular disease; CEL, Ne-(carboxyethyl)lysine; CIMT, carotid intima-media thickness; CML, N $\varepsilon$ (carboxymethyl)lysine; IGM, impaired glucose metabolism; MG-H1, 5-hydro-5-methylimidazolone; NGM, normal glucose metabolism; T2DM, type 2 diabetes. ${ }^{a}$ Normo-, micro- and macroalbuminuria. ${ }^{b}$ Defined as SBP/DBP $\geq 140 / 90 \mathrm{~mm} \mathrm{Hg}$ and/or use of BP-lowering medication. 'Refers to data that are confined to the CODAM Study population only. 


\section{RESULTS}

Compared with participants from the CODAM study, and as has been published before, ${ }^{26,}$

${ }^{36}$ the Hoorn study population was older, included fewer men but more individuals with T2DM, hypertension and CVD and had higher levels of cholesterol, CIMT, plasma CEL and pentosidine, and lower plasma CML (data not shown).

General characteristics of the study population stratified according to prevalent CVD or glucose metabolism status are shown in Table 8.1. Individuals with prior CVD were older, more often had diabetes, had higher levels of triglycerides, BP and CIMT, but had lower eGFR and $A B I$, and more often used BP- and lipid-lowering medication than those without CVD. In addition, BMI, waist circumference, fasting plasma glucose, HbA1c, BP, $\mathrm{CIMT}$ and use of BP-, lipid- and glucose-lowering medication increased and $\mathrm{ABI}$ decreased with deteriorating glucose metabolism status.

\section{COMPARISON OF PLASMA AGE LEVELS BY GLUCOSE METABOLISM STATUS}

Crude plasma levels of protein-bound CML decreased and of protein-bound and free CEL increased with deteriorating levels of glucose metabolism, whereas crude levels of protein-bound pentosidine and free MG-H1 did not differ significantly across these groups (Table 8.1).

After adjustment for cohort, age and sex (Table 8.2, model 1), protein-bound CML remained significantly lower with deteriorating glucose metabolism status [-0.299 SD (95\% Cl: $-0.435 ;-0.164)$ and -0.455 SD (-0.579;-0.331) in IGM and T2DM vs. NGM, respectively] and free CEL remained significantly higher in individuals with T2DM, but not IGM, vs. NGM [0.215 SD (0.018;0.412)]. After additional adjustment for the other study covariates (model 2), the levels of protein-bound CML and free CEL no longer differed significantly across levels of glucose metabolism status. However, but only after full adjustment for study covariates, protein-bound pentosidine was higher in individuals with T2DM vs. NGM [0.152 SD (0.007;0.297)], an increase that was not clearly linear across deteriorating glucose metabolism levels ( $p$ for linear trend=0.060, Table 8.2). 
Table 8.2 Comparisons of levels of plasma protein-bound and free AGEs across levels of glucose metabolism

\begin{tabular}{|c|c|c|c|c|c|c|c|c|}
\hline \multirow[b]{2}{*}{ Dependent variable } & \multirow[b]{2}{*}{ Model } & \multicolumn{3}{|c|}{ IGM vs. NGM } & \multicolumn{3}{|c|}{ T2DM vs. NGM } & \multirow[b]{2}{*}{ p-linear trend } \\
\hline & & $\beta$ & $95 \% \mathrm{Cl}$ & $p$ & $\beta$ & $95 \% \mathrm{Cl}$ & $p$ & \\
\hline \multicolumn{9}{|l|}{ Protein-bound } \\
\hline \multirow[t]{2}{*}{$\log _{e} C M L$} & 1 & -0.299 & $-0.435 ;-0.164$ & $<0.001$ & -0.455 & $-0.579 ;-0.331$ & $<0.001$ & $<0.001$ \\
\hline & 2 & -0.090 & $-0.219 ; 0.040$ & 0.175 & -0.121 & $-0.262 ; 0.020$ & 0.093 & 0.078 \\
\hline \multirow[t]{2}{*}{$\log _{e} C E L$} & 1 & 0.097 & $-0.042 ; 0.236$ & 0.173 & -0.022 & $-0.149 ; 0.105$ & 0.735 & 0.829 \\
\hline & 2 & 0.055 & $-0.088 ; 0.198$ & 0.449 & -0.090 & $-0.246 ; 0.066$ & 0.258 & 0.352 \\
\hline \multirow[t]{2}{*}{ Log $_{\mathrm{e}}$ Pentosidine } & 1 & -0.155 & $-0.291 ;-0.019$ & 0.026 & 0.018 & $-0.106 ; 0.142$ & 0.772 & 0.922 \\
\hline & 2 & -0.012 & $-0.145 ; 0.121$ & 0.861 & 0.152 & $0.007 ; 0.297$ & 0.040 & 0.060 \\
\hline \multicolumn{9}{|l|}{ Free $^{a}$} \\
\hline \multirow[t]{2}{*}{$\log _{e} C M L$} & 1 & -0.119 & $-0.323 ; 0.085$ & 0.253 & -0.083 & $-0.287 ; 0.112$ & 0.404 & 0.329 \\
\hline & 2 & -0.124 & $-0.326 ; 0.077$ & 0.228 & -0.128 & $-0.380 ; 0.124$ & 0.321 & 0.223 \\
\hline \multirow[t]{2}{*}{$\log _{e} C E L$} & 1 & -0.061 & $-0.267 ; 0.145$ & 0.561 & 0.215 & $0.018 ; 0.412$ & 0.033 & 0.060 \\
\hline & 2 & -0.099 & $-0.302 ; 0.105$ & 0.342 & 0.070 & $-0.183 ; 0.323$ & 0.588 & 0.866 \\
\hline \multirow[t]{2}{*}{$\log _{\mathrm{e}} \mathrm{MG}-\mathrm{H} 1$} & 1 & -0.168 & $-0.373 ; 0.037$ & 0.108 & -0.098 & $-0.294 ; 0.099$ & 0.329 & 0.236 \\
\hline & 2 & -0.171 & $-0.380 ; 0.038$ & 0.100 & -0.134 & $-0.387 ; 0.119$ & 0.301 & 0.165 \\
\hline
\end{tabular}

$\beta$, standardized regression coefficient: indicates the difference in plasma protein-bound or free AGEs (in SD) between individuals with impaired glucose metabolism (IGM) or type 2 diabetes (T2DM) vs. normal glucose metabolism (NGM). Model 1: adjusted for cohort (in analyses with protein-bound AGEs only), age and sex; Model 2: model 1 + waist, smoking, MAP, total-to-HDL-cholesterol ratio, Logetriglycerides, eGFR, albuminuria (normo-, micro-,or macro), and use of BP-, lipid- and glucose-lowering medication. ${ }^{\text {a }}$ efers to data that are confined to the CODAM Study population. 


\section{ASSOCIATIONS OF PLASMA AGES WITH CVD}

In the whole study population, crude levels of protein-bound CEL and pentosidine and of all free AGEs were significantly higher, whereas levels of protein-bound CML were significantly lower in individuals with compared to those without CVD (Table 8.1). However, after adjustment for cohort, age, sex, glucose metabolism status and study covariates (Table 8.3, model 2), none of the protein-bound and free AGEs were significantly associated with prevalent CVD.

In analyses stratified according to glucose metabolism status and after full adjustment for covariates, we only found a significant positive association of proteinbound CEL with CVD in individuals with IGM [OR=1.37 (95\% Cl: 1.04;1.81)], but a negative association between this AGE and CVD in individuals with T2DM [OR=0.79 (0.63;0.99)]. Plasma levels of the other AGEs were not significantly associated with CVD (Table 8.3).

\section{ASSOCIATIONS OF PLASMA AGES WITH CIMT OR ABI}

In the whole study population and within strata of glucose metabolism status, none of the plasma AGEs were independently associated with CIMT (Table 8.4, models 1 and 2).

Plasma protein-bound CEL and pentosidine, and free CEL and MG-H1, were also not independently associated with $A B I$ (Table 8.5). However, both protein-bound and free $\mathrm{CML}$ were positively associated with $\mathrm{ABI}$ in analyses adjusted for age, sex, cohort and glucose metabolism status (Table 8.5, model 1), but after further adjustment for other study covariates only the associations between free $\mathrm{CML}$ and $\mathrm{ABI}$ remained statistically significant [0.106 SD (95\% Cl: 0.064;0.148)] (model 2). In analyses stratified by glucose metabolism status, plasma levels of protein-bound and free AGEs were not significantly associated with $\mathrm{ABI}$. 
Table 8.3 Associations of plasma protein-bound and free AGEs with prevalent CVD in the total population and stratified according to glucose metabolism status

\begin{tabular}{|c|c|c|c|c|c|c|c|c|c|c|c|c|c|}
\hline \multirow[b]{2}{*}{ Independent variable } & \multirow[b]{2}{*}{ Model } & \multicolumn{3}{|c|}{$\begin{array}{c}\text { Total population } \\
(n=1,291)\end{array}$} & \multicolumn{3}{|c|}{$\begin{array}{c}\text { NGM } \\
(n=573)\end{array}$} & \multicolumn{3}{|c|}{$\begin{array}{c}\text { IGM } \\
(n=304)\end{array}$} & \multicolumn{3}{|c|}{$\begin{array}{l}\text { T2DM } \\
(n=414)\end{array}$} \\
\hline & & OR & $95 \% \mathrm{Cl}$ & $p$ & OR & $95 \% \mathrm{Cl}$ & $p$ & OR & $95 \% \mathrm{Cl}$ & $p$ & OR & $95 \% \mathrm{Cl}$ & $p$ \\
\hline \multicolumn{14}{|l|}{ Protein-bound } \\
\hline \multirow[t]{2}{*}{$\log _{\mathrm{e}} \mathrm{CML}$} & 1 & 0.89 & $0.79 ; 1.01$ & 0.064 & 0.96 & $0.78 ; 1.17$ & 0.673 & 0.92 & $0.69 ; 1.23$ & 0.568 & 0.81 & $0.67 ; 0.98$ & 0.028 \\
\hline & 2 & 0.97 & $0.85 ; 1.12$ & 0.711 & 1.04 & $0.82 ; 1.33$ & 0.726 & 1.15 & $0.82 ; 1.62$ & 0.430 & 0.85 & $0.68 ; 1.06$ & 0.142 \\
\hline \multirow[t]{2}{*}{$\log _{e} C E L$} & 1 & 1.04 & $0.93 ; 1.17$ & 0.505 & 1.00 & $0.83 ; 1.22$ & 0.973 & 1.37 & $1.07 ; 1.76$ & 0.013 & 0.90 & $0.74 ; 1.10$ & 0.313 \\
\hline & 2 & 1.00 & $0.88 ; 1.14$ & 0.982 & 1.07 & $0.86 ; 1.32$ & 0.555 & 1.37 & $1.04 ; 1.81$ & 0.025 & 0.79 & $0.63 ; 0.99$ & 0.038 \\
\hline \multirow[t]{2}{*}{$\log _{\mathrm{e}}$ Pentosidine } & 1 & 1.04 & $0.92 ; 1.17$ & 0.587 & 1.22 & $0.99 ; 1.49$ & 0.059 & 0.83 & $0.62 ; 1.12$ & 0.218 & 0.98 & $0.82 ; 1.18$ & 0.983 \\
\hline & 2 & 1.07 & $0.93 ; 1.22$ & 0.353 & 1.17 & $0.92 ; 1.48$ & 0.202 & 1.01 & $0.75 ; 1.36$ & 0.961 & 1.04 & $0.84 ; 1.28$ & 0.740 \\
\hline Free $^{a}$ & & & $n=554^{\mathrm{a}}$ & & & $n=287^{\mathrm{a}}$ & & & $n=123^{\mathrm{a}}$ & & & $n=144^{a}$ & \\
\hline \multirow[t]{2}{*}{$\log _{e} C M L$} & 1 & 1.08 & $0.88 ; 1.32$ & 0.467 & 1.21 & $0.90 ; 1.62$ & 0.201 & 0.98 & $0.65 ; 1.47$ & 0.902 & 0.96 & $0.66 ; 1.40$ & 0.829 \\
\hline & 2 & 0.96 & $0.76 ; 1.21$ & 0.709 & 1.09 & $0.78 ; 1.53$ & 0.603 & 1.00 & $0.79 ; 1.28$ & 0.998 & 0.63 & $0.37 ; 1.08$ & 0.095 \\
\hline \multirow[t]{2}{*}{$\log _{e} C E L$} & 1 & 1.12 & $0.92 ; 1.36$ & 0.277 & 1.30 & $0.97 ; 1.73$ & 0.079 & 0.94 & $0.60 ; 1.47$ & 0.770 & 1.00 & $0.71 ; 1.42$ & 0.996 \\
\hline & 2 & 1.01 & $0.81 ; 1.27$ & 0.915 & 1.12 & $0.86 ; 1.68$ & 0.291 & 1.04 & $0.80 ; 1.36$ & 0.880 & 0.72 & $0.45 ; 1.15$ & 0.174 \\
\hline \multirow[t]{2}{*}{$\log _{\mathrm{e}} \mathrm{MG}-\mathrm{H} 1$} & 1 & 1.31 & $1.07 ; 1.60$ & 0.009 & 1.37 & $1.00 ; 1.88$ & 0.048 & 1.21 & $0.80 ; 1.83$ & 0.375 & 1.25 & $0.89 ; 1.75$ & 0.199 \\
\hline & 2 & 1.21 & $0.96 ; 1.53$ & 0.101 & 1.29 & $0.90 ; 1.86$ & 0.175 & 1.24 & $0.97 ; 1.58$ & 0.377 & 1.08 & $0.69 ; 1.68$ & 0.741 \\
\hline
\end{tabular}

OR, odds ratio: indicates the odds of prevalent CVD per SD increase in plasma protein-bound or free AGEs. Model 1: adjusted for cohort (in analyses with protein-bound AGEs only), age, sex and glucose metabolism status (in analyses in the total population only); Model 2: model 1 + waist, smoking, MAP, total-to-HDL-cholesterol ratio, Logetriglycerides, eGFR, albuminuria (normo-, micro- or macro), BP-, lipid- and/or glucose-lowering medication. ${ }^{\mathrm{a}}$ Refers to data that are confined to the CODAM Study population. 


\section{DISCUSSION}

This study had two main findings. First, after adjustments for potential confounders, plasma levels of protein-bound and free CML and CEL and of free MG-H1 were not increased among individuals with increasing deteriorating glucose metabolism status. Only levels of protein-bound pentosidine were increased, among individuals with T2DM only. Second, overall, none of the protein-bound and free AGEs were independently associated with prevalent CVD or the surrogate markers of atherosclerosis examined herein, with the exception of free levels of $C M L$, which were positively associated with $A B I$.

Previous studies comparing plasma levels of AGEs between individuals with vs. without T2DM have resulted in inconsistent findings as several studies have shown these to be increased in T2DM ${ }^{10,12,37-39}$ or not to differ between groups. ${ }^{9,11,13,16,39-41}$ Our current findings are, for the most part, in agreement with the latter studies and extend these by being obtained in a larger sample and with state-of-the-art methodology for AGE measurements.

The higher levels of pentosidine observed among individuals with T2DM seemed to be in agreement with earlier observations, ${ }^{42}$ but we cannot exclude the possibility that this was due to chance, as we did not find any trend for increases in pentosidine in IGM vs. NGM. Furthermore, the levels of pentosidine were only significantly higher among individuals with T2DM vs. NGM after adjustment for study covariates, that is, after negative confounding by mainly waist circumference, blood lipids and GFR was taken into account. In addition, we observed a decrease of plasma protein-bound $\mathrm{CML}$ in individuals with IGM and T2DM compared with those with NGM, which is consistent with a recent report. ${ }^{43}$ These decreases with deteriorating glucose metabolism status may, at least in part, be attributed to the higher levels of obesity in individuals with IGM and T2DM individuals as previous studies have shown inverse associations between measures of obesity (e.g. BMI) and plasma protein-bound CML. ${ }^{44-46}$ Indeed, also in the present study BMI [standardized $\beta=-0.222$ (95\% Cl: -0.277 to -0.166$)$ ] and waist circumference [-0.307 (95\% Cl: -0.366 to -0.248 )] were inversely associated with protein-bound $\mathrm{CML}$ and the differences in protein-bound CML across deteriorating glucose metabolism status were importantly attenuated after additional adjustments for covariates including waist (models 2 vs. 1, Table 8.2) or BMI (data not shown). In addition, we found positive associations of protein-bound $\mathrm{CML}$ and more strongly so of free $\mathrm{CML}$ with $\mathrm{ABI}$. Although both low (usually set at $<0.9$ ) and high (usually set at $>1.4$ ) $\mathrm{ABI}$ are associated with increased risk for incident $C V D,{ }^{47}$ the current associations may, if anything, be considered beneficial, as individuals with high $\mathrm{ABI}(>1.4, n=7)$ were excluded from the current analyses. 
Table 8.4 Associations of plasma protein-bound and free AGEs with CIMT in the total population and stratified according to glucose metabolism status

\begin{tabular}{|c|c|c|c|c|c|c|c|c|c|c|c|c|c|}
\hline \multirow[b]{2}{*}{ Independent variable } & \multirow[b]{2}{*}{ Model } & \multicolumn{3}{|c|}{$\begin{array}{c}\text { Total population } \\
(n=1,182)\end{array}$} & \multicolumn{3}{|c|}{$\begin{array}{c}\mathrm{NGM} \\
(n=550)\end{array}$} & \multicolumn{3}{|c|}{$\begin{array}{c}\text { IGM } \\
(n=266)\end{array}$} & \multicolumn{3}{|c|}{$\begin{array}{c}\text { T2DM } \\
(n=366)\end{array}$} \\
\hline & & $\beta$ & $95 \% \mathrm{Cl}$ & $p$ & $\beta$ & $95 \% \mathrm{Cl}$ & $p$ & $\beta$ & $95 \% \mathrm{Cl}$ & $p$ & $\beta$ & $95 \% \mathrm{Cl}$ & $p$ \\
\hline \multicolumn{14}{|l|}{ Protein-bound } \\
\hline \multirow[t]{2}{*}{$\log _{e} C M L$} & 1 & -0.052 & $-0.108 ; 0.003$ & 0.065 & -0.080 & $-0.165 ; 0.005$ & 0.064 & 0.017 & $-0.114 ; 0.147$ & 0.801 & -0.045 & $-0.139 ; 0.050$ & 0.353 \\
\hline & 2 & -0.027 & $-0.086 ; 0.032$ & 0.367 & -0.037 & $-0.128 ; 0.053$ & 0.424 & 0.021 & $-0.116 ; 0.157$ & 0.767 & -0.021 & $-0.120 ; 0.079$ & 0.681 \\
\hline \multirow[t]{2}{*}{$\log _{e} C E L$} & 1 & 0.027 & $-0.027 ; 0.080$ & 0.330 & 0.023 & $-0.058 ; 0.103$ & 0.577 & 0.053 & $-0.056 ; 0.163$ & 0.339 & 0.007 & $-0.091 ; 0.105$ & 0.889 \\
\hline & 2 & 0.034 & $-0.020 ; 0.088$ & 0.214 & 0.032 & $-0.010 ; 0.073$ & 0.447 & 0.064 & $-0.044 ; 0.173$ & 0.245 & 0.021 & $-0.078 ; 0.121$ & 0.676 \\
\hline \multirow[t]{2}{*}{ Log $_{\mathrm{e}}$ Pentosidine } & 1 & -0.049 & $-0.105 ; 0.006$ & 0.079 & -0.067 & $-0.153 ; 0.020$ & 0.129 & -0.008 & $-0.132 ; 0.116$ & 0.900 & -0.042 & $-0.134 ; 0.050$ & 0.372 \\
\hline & 2 & -0.047 & $-0.105 ; 0.010$ & 0.108 & -0.048 & $-0.137 ; 0.041$ & 0.298 & -0.009 & $-0.139 ; 0.121$ & 0.894 & -0.025 & $-0.120 ; 0.069$ & 0.599 \\
\hline Free $^{a}$ & & & $n=516^{\mathrm{a}}$ & & & $n=275^{a}$ & & & $n=110^{\mathrm{a}}$ & & & $n=131^{\mathrm{a}}$ & \\
\hline \multirow[t]{2}{*}{$\log _{e} C M L$} & 1 & -0.056 & $-0.142 ; 0.031$ & 0.207 & -0.031 & $-0.146 ; 0.085$ & 0.603 & -0.150 & $-0.327 ; 0.027$ & 0.097 & -0.042 & $-0.230 ; 0.146$ & 0.659 \\
\hline & 2 & -0.077 & $-0.166 ; 0.012$ & 0.089 & -0.046 & $-0.161 ; 0.068$ & 0.433 & -0.144 & $-0.327 ; 0.039$ & 0.124 & -0.125 & $-0.337 ; 0.088$ & 0.249 \\
\hline \multirow[t]{2}{*}{$\log _{e} C E L$} & 1 & 0.034 & $-0.051 ; 0.119$ & 0.434 & 0.062 & $-0.053 ; 0.177$ & 0.289 & -0.099 & $-0.293 ; 0.096$ & 0.320 & 0.054 & $-0.117 ; 0.224$ & 0.537 \\
\hline & 2 & 0.012 & $-0.076 ; 0.100$ & 0.796 & 0.042 & $-0.074 ; 0.158$ & 0.480 & -0.137 & $-0.338 ; 0.064$ & 0.189 & -0.013 & $-0.199 ; 0.173$ & 0.891 \\
\hline \multirow[t]{2}{*}{$\log _{e} M G-H 1$} & 1 & 0.022 & $-0.065 ; 0.108$ & 0.624 & -0.036 & $-0.157 ; 0.084$ & 0.555 & 0.072 & $-0.107 ; 0.251$ & 0.429 & 0.084 & $-0.085 ; 0.254$ & 0.331 \\
\hline & 2 & 0.003 & $-0.086 ; 0.092$ & 0.946 & -0.023 & $-0.109 ; 0.063$ & 0.713 & 0.052 & $-0.131 ; 0.234$ & 0.580 & 0.035 & $-0.156 ; 0.226$ & 0.720 \\
\hline
\end{tabular}

$\beta$, standardized regression coefficient: indicates the difference (in SD) in CIMT per SD increase in plasma protein-bound or free AGEs. Model 1: adjusted for cohort (in analyses with protein-bound AGEs only), age, sex and glucose metabolism status (in analyses in the total population only); Model 2: model $1+$ waist, smoking, MAP, total-to-HDL-cholesterol ratio, Logetriglycerides, eGFR, albuminuria (normo-, micro- or macro), BP-, lipid- and glucose-lowering medication. aRefers to data that are confined to the CODAM Study population. 
Table 8.5 Associations of plasma protein-bound and free AGEs with ABI in the total population and stratified according to glucose metabolism status

\begin{tabular}{|c|c|c|c|c|c|c|c|c|c|c|c|c|c|}
\hline \multirow[b]{2}{*}{ Independent variable } & \multirow[b]{2}{*}{ Model } & \multicolumn{3}{|c|}{$\begin{array}{c}\text { Total population } \\
(n=1,122)\end{array}$} & \multicolumn{3}{|c|}{$\begin{array}{c}\text { NGM } \\
(n=570)\end{array}$} & \multicolumn{3}{|c|}{$\begin{array}{c}\text { IGM } \\
(n=297)\end{array}$} & \multicolumn{3}{|c|}{$\begin{array}{l}\text { T2DM } \\
(n=262)\end{array}$} \\
\hline & & $\beta$ & $95 \% \mathrm{Cl}$ & $p$ & $\beta$ & $95 \% \mathrm{Cl}$ & $p$ & $\beta$ & $95 \% \mathrm{Cl}$ & $p$ & $\beta$ & $95 \% \mathrm{Cl}$ & $p$ \\
\hline \multicolumn{14}{|l|}{ Protein-bound } \\
\hline \multirow[t]{2}{*}{$\log _{e} C M L$} & 1 & 0.062 & $0.005 ; 0.118$ & 0.032 & 0.057 & $-0.020 ; 0.135$ & 0.146 & 0.029 & $-0.091 ; 0.150$ & 0.633 & 0.077 & $-0.066 ; 0.220$ & 0.291 \\
\hline & 2 & 0.042 & $-0.018 ; 0.101$ & 0.168 & 0.035 & $-0.048 ; 0.117$ & 0.410 & 0.043 & $-0.088 ; 0.173$ & 0.521 & 0.062 & $-0.089 ; 0.213$ & 0.422 \\
\hline \multirow[t]{2}{*}{$\log _{\mathrm{e}} \mathrm{CEL}$} & 1 & -0.053 & $-0.107 ; 0.001$ & 0.054 & -0.007 & $-0.080 ; 0.066$ & 0.845 & -0.046 & $-0.148 ; 0.056$ & 0.375 & -0.098 & $-0.249 ; 0.053$ & 0.204 \\
\hline & 2 & -0.049 & $-0.102 ; 0.004$ & 0.068 & -0.023 & $-0.096 ; 0.049$ & 0.528 & -0.028 & $-0.132 ; 0.076$ & 0.602 & -0.065 & $-0.214 ; 0.084$ & 0.393 \\
\hline \multirow[t]{2}{*}{$\log _{\mathrm{e}}$ Pentosidine } & 1 & 0.014 & $-0.042 ; 0.070$ & 0.624 & -0.057 & $-0.136 ; 0.022$ & 0.156 & 0.114 & $-0.005 ; 0.233$ & 0.061 & 0.063 & $-0.066 ; 0.191$ & 0.338 \\
\hline & 2 & 0.008 & $-0.050 ; 0.065$ & 0.790 & -0.052 & $-0.134 ; 0.030$ & 0.218 & 0.112 & $-0.017 ; 0.240$ & 0.088 & 0.047 & $-0.085 ; 0.180$ & 0.484 \\
\hline \multicolumn{2}{|l|}{ Free $^{a}$} & & $n=550^{\mathrm{a}}$ & & & $n=287^{\mathrm{a}}$ & & & $n=122^{\mathrm{a}}$ & & & $n=141^{\mathrm{a}}$ & \\
\hline \multirow[t]{2}{*}{$\log _{e} C M L$} & 1 & 0.083 & $0.005 ; 0.162$ & 0.037 & 0.075 & $-0.027 ; 0.177$ & 0.148 & 0.090 & $-0.041 ; 0.221$ & 0.179 & 0.092 & $-0.103 ; 0.288$ & 0.355 \\
\hline & 2 & 0.106 & $0.064 ; 0.148$ & 0.011 & 0.091 & $0.037 ; 0.144$ & 0.092 & 0.119 & $-0.018 ; 0.257$ & 0.090 & 0.140 & $-0.073 ; 0.353$ & 0.200 \\
\hline \multirow[t]{2}{*}{$\log _{e} C E L$} & 1 & 0.035 & $-0.043 ; 0.113$ & 0.380 & 0.069 & $-0.032 ; 0.169$ & 0.182 & 0.057 & $-0.085 ; 0.199$ & 0.432 & -0.045 & $-0.230 ; 0.140$ & 0.633 \\
\hline & 2 & 0.055 & $-0.013 ; 0.096$ & 0.190 & 0.095 & $0.041 ; 0.148$ & 0.075 & 0.089 & $0.012 ; 0.166$ & 0.249 & -0.021 & $-0.216 ; 0.173$ & 0.831 \\
\hline \multirow[t]{2}{*}{$\log _{\mathrm{e}} \mathrm{MG}-\mathrm{H} 1$} & 1 & 0.014 & $-0.065 ; 0.092$ & 0.730 & 0.014 & $-0.093 ; 0.120$ & 0.799 & 0.058 & $-0.075 ; 0.191$ & 0.394 & -0.019 & $-0.198 ; 0.160$ & 0.833 \\
\hline & 2 & 0.036 & $-0.006 ; 0.078$ & 0.389 & 0.040 & $-0.016 ; 0.096$ & 0.477 & 0.086 & $-0.052 ; 0.223$ & 0.222 & 0.006 & $-0.158 ; 0.169$ & 0.953 \\
\hline
\end{tabular}

$\beta$, standardized regression coefficient: indicates the difference (in SD) in ABI per SD increase in plasma protein-bound or free AGEs. Model 1: adjusted for cohort (in analyses with protein-bound AGEs only), age, sex and glucose metabolism status (in analyses in the total population only); Model 2: model $1+$ waist, smoking, MAP, total-to-HDL-cholesterol ratio, Logetriglycerides, eGFR, albuminuria (normo-, micro- or macro), BP-, lipid- and glucose-lowering medication. aRefers to data that are confined to the CODAM Study population only. 
A general appraisal of the literature on the associations between plasma AGEs and (surrogate markers of) CVD shows inconsistent findings, i.e. both positive ${ }^{9,10,14-17}$ and lack of associations ${ }^{11,12,18}$ between plasma AGEs and CVD have been described. These differences could be due to differences between studies in sample size (i.e. some were rather small ${ }^{9}, 10,12,14$ ) and/or inconsistent or incomplete consideration of potential confounding factors such as age and/or GFR. ${ }^{10,12,14,18}$ Our findings describe a lack of independent associations between plasma AGEs and CVD, CIMT and ABI (except for free CML with the latter) after adjustment for a large set of potential confounding factors, and are thus consistent with some of the previous studies. ${ }^{11,12,18}$ Our study thus illustrates the importance of these adjustments in cohort studies, because drawing inferences from unadjusted data would have led to different and misleading conclusions: e.g. that individuals with CVD had significantly lower levels of CML and higher levels of all remaining AGEs investigated herein (on the basis of the unadjusted data reported in Table 1). Our study also extends previous ones by investigating the consistency of the associations within strata of deteriorating glucose metabolism. For instance, we found that plasma protein-bound CEL was positively associated with CVD in individuals with IGM, but inversely associated with CVD in individuals with T2DM. These contrasting associations are difficult to conciliate and most likely reflect a chance finding.

Several biological factors might explain the apparent contrasting findings between our study and the experimental work that has supported a causal role of AGEs in the development of (diabetes-related) vascular complications. First, many factors, such as dietary intake of AGEs, ${ }^{48}$ ageing, ${ }^{49,} 50$ renal function ${ }^{51}$ or use of lipid-lowering medication ${ }^{6}$ may influence the concentrations of AGEs in tissues and plasma. Consequently, the origin and degradation of AGEs in tissues, circulating cells and plasma may differ between populations. In addition, intracellular glycation, via production of methylglyoxal, is believed to be the major source of AGEs ${ }^{52}$ and not all AGEs may end up in the circulation. Consequently, plasma levels of AGEs may not adequately represent tissue AGE accumulation.

Despite the large sample size, the use of state-of-the-art methodology for assessment of a wide range of both protein-bound and free AGEs in plasma, and the extensive adjustment for potential confounders, our study has some limitations. Firstly, the study population consists of a middle-aged Caucasian population at high risk for (or with prevalent) T2DM and CVD. Our findings may thus not apply to the general Caucasian population or to other ethnicities. Secondly, given the cross-sectional design of our study, we cannot rule out the lack of involvement of plasma AGEs in the development of incident CVD in other populations or disease states. Indeed, we have previously shown that higher levels of protein-bound AGEs as examined herein were associated with higher risk for incident CVD and all-cause mortality among individuals with type 1 diabetes. ${ }^{53}$ Finally, we were only able to ascertain levels of free AGEs and investigate their associations with 
study outcomes in one of the sub-populations (CODAM). However, the associations of the protein-bound AGEs, which were assessed in both the Hoorn and the CODAM studies, did not differ between cohorts. This suggests that the findings regarding the free AGEs are likely to also apply to the whole study population examined herein, which enabled comparison of associations of several plasma AGEs in a single study. To our knowledge, our study is one of the few large cohort studies investigating the associations between several plasma protein-bound and free AGEs (measured with state-of-the-art techniques) and markers of CVD.

In conclusion, plasma AGEs are not associated with the prevalence of CVD, CIMT and $A B I$ in two large cohorts of individuals with NGM, IGM and T2DM. Although we cannot fully exclude the importance of AGEs in the pathogenesis of CVD on the basis of these findings, our study suggests that AGE levels other than those assessed in plasma may serve as better biomarkers for increased CVD risk in a population-based setting. Therefore, novel alternative measurements of AGEs, such as free methylglyoxal, AGE concentrations in circulating cells or tissue AGE accumulation (e.g. by skin autofluorescence), which likely better reflect the AGE production in tissues, need to be further investigated for their added value in the evaluation of the role of AGEs in CVD in large cohort studies. 


\section{REFERENCES}

1. Nakamura Y, Horii Y, Nishino T, Shiiki H, Sakaguchi Y, Kagoshima T, et al. Immunohistochemical localization of advanced glycosylation end products in coronary atheroma and cardiac tissue in diabetes mellitus. Am J Pathol. 1993;143:1649-1656

2. Baidoshvili A, Krijnen PA, Kupreishvili K, Ciurana C, Bleeker W, Nijmeijer R, et al. N(epsilon)(carboxymethyl)lysine depositions in intramyocardial blood vessels in human and rat acute myocardial infarction: A predictor or reflection of infarction? Arterioscler Thromb Vasc Biol. 2006;26:2497-2503

3. Schleicher ED, Wagner E, Nerlich AG. Increased accumulation of the glycoxidation product $n(e p s i l o n)-$ (carboxymethyl)lysine in human tissues in diabetes and aging. J Clin Invest. 1997;99:457-468

4. Watson AM, Soro-Paavonen A, Sheehy K, Li J, Calkin AC, Koitka A, et al. Delayed intervention with age inhibitors attenuates the progression of diabetes-accelerated atherosclerosis in diabetic apolipoprotein $\mathrm{e}$ knockout mice. Diabetologia. 2011;54:681-689

5. Harja E, Bu DX, Hudson BI, Chang JS, Shen X, Hallam K, et al. Vascular and inflammatory stresses mediate atherosclerosis via rage and its ligands in apoe-/- mice. J Clin Invest. 2008;118:183-194

6. Cuccurullo C, lezzi A, Fazia ML, De Cesare D, Di Francesco A, Muraro R, et al. Suppression of rage as a basis of simvastatin-dependent plaque stabilization in type 2 diabetes. Arterioscler Thromb Vasc Biol. 2006;26:2716-2723

7. Goldin A, Beckman JA, Schmidt AM, Creager MA. Advanced glycation end products: Sparking the development of diabetic vascular injury. Circulation. 2006;114:597-605

8. Brownlee M. Biochemistry and molecular cell biology of diabetic complications. Nature. 2001;414:813-820

9. Sugiyama S, Miyata T, Ueda Y, Tanaka H, Maeda K, Kawashima S, et al. Plasma levels of pentosidine in diabetic patients: An advanced glycation end product. J Am Soc Nephrol. 1998;9:1681-1688

10. Yoshida N, Okumura K, Aso Y. High serum pentosidine concentrations are associated with increased arterial stiffness and thickness in patients with type 2 diabetes. Metabolism. 2005;54:345-350

11. Busch M, Franke S, Wolf G, Brandstadt A, Ott U, Gerth J, et al. The advanced glycation end product $\mathrm{n}$ (epsilon)-carboxymethyllysine is not a predictor of cardiovascular events and renal outcomes in patients with type 2 diabetic kidney disease and hypertension. Am J Kidney Dis. 2006;48:571-579

12. Kilhovd BK, Berg TJ, Birkeland KI, Thorsby P, Hanssen KF. Serum levels of advanced glycation end products are increased in patients with type 2 diabetes and coronary heart disease. Diabetes Care.1999;22:15431548

13. Kilhovd BK, Juutilainen A, Lehto S, Ronnemaa T, Torjesen PA, Hanssen KF, et al. Increased serum levels of methylglyoxal-derived hydroimidazolone-age are associated with increased cardiovascular disease mortality in nondiabetic women. Atherosclerosis. 2009;205:590-594

14. Dworacka M, Winiarska H, Szymanska M, Szczawinska K, Wierusz-Wysocka B. Serum n-epsilon(carboxymethyl)lysine is elevated in nondiabetic coronary heart disease patients. J Basic Clin Physiol Pharmacol. 2002;13:201-213

15. Takahashi R, Imamura A, Yoshikane M, Suzuki M, Murakami R, Cheng XW, et al. High serum concentrations of pentosidine, an advanced glycation end product, are associated with low normal value of ankle-brachial index in apparently healthy men. Metabolism. 2011;60:649-654

16. Semba RD, Ferrucci L, Sun K, Beck J, Dalal M, Varadhan R, et al. Advanced glycation end products and their circulating receptors predict cardiovascular disease mortality in older community- dwelling women. Aging Clin Exp Res. 2009;21:182-190

17. Semba RD, Bandinelli S, Sun K, Guralnik JM, Ferrucci L. Plasma carboxymethyl-lysine, an advanced glycation end product, and all-cause and cardiovascular disease mortality in older community-dwelling adults. J Am Geriatr Soc. 2009;57:1874-1880

18. Baumann M, Richart T, Sollinger D, Pelisek J, Roos M, Kouznetsova T, et al. Association between carotid diameter and the advanced glycation end product $\mathrm{n}$-epsilon-carboxymethyllysine (cml). Cardiovasc Diabetol. 2009;8:45

19. Scheijen JL, van de Waarenburg MP, Stehouwer CD, Schalkwijk CG. Measurement of pentosidine in human plasma protein by a single-column high-performance liquid chromatography method with fluorescence detection. J Chromatogr B Analyt Technol Biomed Life Sci. 2009;877:610-614 
20. Teerlink T, Barto R, Ten Brink HJ, Schalkwijk CG. Measurement of nepsilon-(carboxymethyl)lysine and nepsilon-(carboxyethyl)lysine in human plasma protein by stable-isotope-dilution tandem mass spectrometry. Clin Chem. 2004;50:1222-1228

21. Thornalley PJ, Battah S, Ahmed N, Karachalias N, Agalou S, Babaei-Jadidi R, et al. Quantitative screening of advanced glycation endproducts in cellular and extracellular proteins by tandem mass spectrometry. Biochem J. 2003;375:581-592

22. Makita Z, Bucala R, Rayfield EJ, Friedman EA, Kaufman AM, Korbet SM, et al. Reactive glycosylation endproducts in diabetic uraemia and treatment of renal failure. Lancet. 1994;343:1519-1522

23. Thewissen MM, Damoiseaux JG, Duijvestijn AM, van Greevenbroek MM, van der Kallen CJ, Feskens EJ, et al. Abdominal fat mass is associated with adaptive immune activation: The codam study. Obesity (Silver Spring). 2011

24. Jacobs M, van Greevenbroek MM, van der Kallen CJ, Ferreira I, Blaak EE, Feskens EJ, et al. Low-grade inflammation can partly explain the association between the metabolic syndrome and either coronary artery disease or severity of peripheral arterial disease: The codam study. Eur J Clin Invest. 2009;39:437-444

25. Henry RM, Kostense PJ, Spijkerman AM, Dekker JM, Nijpels G, Heine RJ, et al. Arterial stiffness increases with deteriorating glucose tolerance status: The hoorn study. Circulation. 2003;107:2089-2095

26. Engelen L, Ferreira I, Brouwers O, Henry RM, Dekker JM, Nijpels G, et al. Polymorphisms in glyoxalase 1 gene are not associated with vascular complications: The hoorn and codam studies. J Hypertens. 2009;27:1399-1403

27. Spijkerman AM, Adriaanse MC, Dekker JM, Nijpels G, Stehouwer CD, Bouter LM, et al. Diabetic patients detected by population-based stepwise screening already have a diabetic cardiovascular risk profile. Diabetes Care. 2002;25:1784-1789

28. Alberti KG, Zimmet PZ. Definition, diagnosis and classification of diabetes mellitus and its complications. Part 1: Diagnosis and classification of diabetes mellitus provisional report of a who consultation. Diabet Med. 1998;15:539-553

29. Brands PJ, Hoeks AP, Willigers J, Willekes C, Reneman RS. An integrated system for the non-invasive assessment of vessel wall and hemodynamic properties of large arteries by means of ultrasound. Eur J Ultrasound. 1999;9:257-266

30. Hoeks AP, Willekes C, Boutouyrie P, Brands PJ, Willigers JM, Reneman RS. Automated detectionof local artery wall thickness based on $\mathrm{m}$-line signal processing. Ultrasound Med Biol. 1997;23:1017-1023

31. Atsma F, Bartelink ML, Grobbee DE, van der Schouw YT. Best reproducibility of the ankle-arm index was calculated using doppler and dividing highest ankle pressure by highest arm pressure. J Clin Epidemiol. 2005;58:1282-1288

32. Levey AS, Bosch JP, Lewis JB, Greene T, Rogers N, Roth D. A more accurate method to estimate glomerular filtration rate from serum creatinine: A new prediction equation. Modification of diet in renal disease study group. Ann Intern Med. 1999;130:461-470

33. Snijder MB, Henry RM, Visser M, Dekker JM, Seidell JC, Ferreira I, et al. Regional body composition as a determinant of arterial stiffness in the elderly: The hoorn study. J Hypertens. 2004;22:2339-2347

34. Sterne JA, White IR, Carlin JB, Spratt M, Royston P, Kenward MG, et al. Multiple imputation for missing data in epidemiological and clinical research: Potential and pitfalls. BMJ. 2009;338:b2393

35. Janssen KJ, Donders AR, Harrell FE, Jr., Vergouwe $Y$, Chen $Q$, Grobbee DE, et al. Missing covariate data in medical research: To impute is better than to ignore. J Clin Epidemiol. 2010;63:721-727

36. Engelen L, Ferreira I, Gaens KH, Henry RM, Dekker JM, Nijpels G, et al. The association between the $-374 t / a$ polymorphism of the receptor for advanced glycation endproducts gene and blood pressure and arterial stiffness is modified by glucose metabolism status: The hoorn and codam studies. J Hypertens. 2010;28:285293

37. Boehm BO, Schilling S, Rosinger S, Lang GE, Lang GK, Kientsch-Engel R, et al. Elevated serum levels of n(epsilon)-carboxymethyl-lysine, an advanced glycation end product, are associated with proliferative diabetic retinopathy and macular oedema. Diabetologia. 2004;47:1376-1379

38. Wautier MP, Massin P, Guillausseau PJ, Huijberts M, Levy B, Boulanger E, et al. N(carboxymethyl)lysine as a biomarker for microvascular complications in type 2 diabetic patients. Diabetes Metab. 2003;29:44-52

39. Daimon M, Ono Y, Saito T, Yamaguchi H, Hirata A, Ohnuma H, et al. Increased serum levels of pentosidine, but not carboxymethyl lysine, in type 2 diabetes without obvious diabetic nephropathy. Diabetes Care. $1999 ; 22: 877-878$ 
40. Endo M, Yanagisawa K, Tsuchida K, Okamoto T, Matsushita T, Higuchi M, et al. Increased levels of vascular endothelial growth factor and advanced glycation end products in aqueous humor of patients with diabetic retinopathy. Horm Metab Res. 2001;33:317-322

41. Wagner Z, Wittmann I, Mazak I, Schinzel R, Heidland A, Kientsch-Engel R, et al. N(epsilon)(carboxymethyl)lysine levels in patients with type 2 diabetes: Role of renal function. Am J Kidney Dis. 2001;38:785-791

42. Lapolla A, Piarulli F, Sartore G, Ceriello A, Ragazzi E, Reitano R, et al. Advanced glycation end products and antioxidant status in type 2 diabetic patients with and without peripheral artery disease. Diabetes Care. 2007;30:670-676

43. Semba RD, Beck J, Sun K, Egan JM, Carlson OD, Varadhan R, et al. Relationship of a dominant advanced glycation end product, serum carboxymethyl-lysine, and abnormal glucose metabolism in adults: The baltimore longitudinal study of aging. J Nutr Health Aging. 2010;14:507-513

44. Semba RD, Arab L, Sun K, Nicklett EJ, Ferrucci L. Fat mass is inversely associated with serum carboxymethyllysine, an advanced glycation end product, in adults. J Nutr. 2011;141:1726-1730

45. Grote VA, Nieters A, Kaaks R, Tjonneland A, Roswall N, Overvad K, et al. The associations of advanced glycation end products and its soluble receptor with pancreatic cancer risk: A case-control study within the prospective epic cohort. Cancer Epidemiol Biomarkers Prev. 2012;21:619-628

46. Sebekova K, Somoza V, Jarcuskova M, Heidland A, Podracka L. Plasma advanced glycation end products are decreased in obese children compared with lean controls. Int J Pediatr Obes. 2009;4:112-118

47. Arain FA, Ye Z, Bailey KR, Chen Q, Liu G, Leibson CL, et al. Survival in patients with poorly compressible leg arteries. J Am Coll Cardiol. 2012;59:400-407

48. Koschinsky T, He CJ, Mitsuhashi T, Bucala R, Liu C, Buenting C, et al. Orally absorbed reactive glycation products (glycotoxins): An environmental risk factor in diabetic nephropathy. Proc Natl Acad Sci U S A. 1997;94:6474-6479

49. Uribarri J, Cai W, Peppa M, Goodman S, Ferrucci L, Striker G, et al. Circulating glycotoxins and dietary advanced glycation endproducts: Two links to inflammatory response, oxidative stress, and aging. J Gerontol A Biol Sci Med Sci. 2007;62:427-433

50. Hamelin M, Borot-Laloi C, Friguet B, Bakala H. Increased level of glycoxidation product $n$ (epsilon)(carboxymethyl)lysine in rat serum and urine proteins with aging: Link with glycoxidative damage accumulation in kidney. Arch Biochem Biophys. 2003;411:215-222

51. Lieuw-A-Fa ML, van Hinsbergh VW, Teerlink T, Barto R, Twisk J, Stehouwer CD, et al. Increased levels of $\mathrm{n}$ (epsilon)-(carboxymethyl)lysine and $\mathrm{n}$ (epsilon)-(carboxyethyl)lysine in type 1 diabetic patients with impaired renal function: Correlation with markers of endothelial dysfunction. Nephrol Dial Transplant. 2004;19:631-636

52. Shinohara M, Thornalley PJ, Giardino I, Beisswenger P, Thorpe SR, Onorato J, et al. Overexpression of glyoxalase-i in bovine endothelial cells inhibits intracellular advanced glycation endproduct formation and prevents hyperglycemia-induced increases in macromolecular endocytosis. J Clin Invest. 1998;101:11421147

53. Nin JW, Jorsal A, Ferreira I, Schalkwijk CG, Prins MH, Parving HH, et al. Higher plasma levels of advanced glycation end products are associated with incident cardiovascular disease and all-cause mortality in type 1 diabetes: A 12-year follow-up study. Diabetes Care.34:442-447 

CHAPTER $\mathbf{g}$

SEEMERAL DISCUSSIOH 


\section{GENERAL DISCUSSION}

The mechanisms underlying the development of micro- and macrovascular complications in diabetes are not completely understood, but the increased formation of advanced glycation endproducts (AGEs), i.e., the glycation pathway, may constitute one such mechanism. ${ }^{1}$ So far, the evidence for the role of the glycation pathway in (diabetesrelated) cardiovascular disease (CVD) has been mainly derived from (animal) experimental studies. The aims of this thesis were, therefore, to evaluate, in a number of epidemiological studies, possible interventions in the glycation pathway (Part 1) and the role of the glycation pathway in the aetiology of [type 2 diabetes (T2DM)-related] CVD (Part 2).

This final chapter, first, briefly summarizes the main findings of the previous chapters and, second, discusses some methodological issues regarding the studies included in this thesis. Third, the potential clinical relevance of the current findings will be addressed and last, some implications for the direction of future research are proposed.

\section{MAIN FINDINGS}

\section{INTERVENTIONS IN THE GLYCATION PATHWAY}

Chapter 2 summarized the current literature on potential AGE-inhibiting effects of different therapies, either specifically developed for AGE inhibition or developed for interventions unrelated to glycation-based theories. Although experimental studies concerning the specific AGE inhibitor aminoguanidine ${ }^{2-13}$ and AGE breaker alagebrium ${ }^{14-22}$ clearly resulted in beneficial effects, it seems that safety and/or efficacy in clinical trials with these compounds are of concern. ${ }^{23-27}$ With regard to the other therapies developed for purposes unrelated to glycation, but which had also shown AGE-inhibiting effects in in vitro and/or animal experimental studies, such as the $B$ vitamins pyridoxamine ${ }^{28-32}$ and thiamine $^{31-36}$ the anti-hyperglycaemic agent metformin ${ }^{37}$ and angiotensin receptor blockers (ARBs), ${ }^{38-52}$ the current evidence is fairly limited due to a large heterogeneity in study designs and/or measurement techniques, which have often been sub-optimal. ${ }^{45-67}$ More large, specific and well-designed studies are needed to fully elucidate their potential AGE-inhibiting effects.

Given the similarities in structure between metformin and aminoguanidine, it had been proposed that metformin detoxifies dicarbonyls (i.e. AGE precursors) in a similar way 
as aminoguanidine, ${ }^{37}$ which could explain, at least in part, the beneficial effects of metformin on cardiovascular outcomes in T2DM. ${ }^{68}$ Previous (observational) studies in individuals with T2DM have indeed shown beneficial 'effects' of metformin on a range of measures of glycation. ${ }^{59-61}$ However, although Chapter 3 confirmed a decrease [-19.3\% $(95 \% \mathrm{Cl}:-23.5 ;-14.8)]$ in levels of the dicarbonyl compound 3-deoxyglucosone (3DG) after treatment with metformin in non-obese patients with T2DM, remarkably, we found a similar decrease [-20.8\% $(-24.9 ;-16.3)]$ in 3DG levels after treatment with another antihyperglycaemic agent, repaglinide. The different working mechanisms of the antihyperglycaemic effects of metformin and repaglinide suggest thus that improvement in glycaemic control, rather than a specific effect of metformin on quenching of dicarbonyls, results in a decrease in 3DG. However, the metformin- and repaglinide-induced reduction in 3DG levels was associated with a reduction in endothelial markers and may as such constitute a metabolic pathway through which both treatments have a beneficial impact on the cardiovascular risk profile.

The inhibiting effects of ARBs on AGEs and their sequelae, as shown in in vitro ${ }^{38}$ and animal experiments, ${ }^{39-44}$ led to the hypothesis that such AGE-inhibiting effects could explain, at least in part, the beneficial effects of ARBs on cardiovascular outcomes in T2DM. ${ }^{69,70}$ However, although several small and mostly uncontrolled clinical studies have indeed shown decreases in circulating ${ }^{45-49}$ or urinary ${ }^{47,49,50}$ AGEs after treatment with ARBs, the large RCT in individuals with T2DM in Chapter 4 investigating the effects of two years of irbesartan treatment on plasma $N^{\varepsilon}$-(carboxymethyl)lysine $(C M L)$ and $N^{\varepsilon}$ (carboxyethyl)lysine (CEL) failed to show this. Our findings are in line with the only two other large RCTs that have investigated this so far, showing no AGE-inhibiting effects of

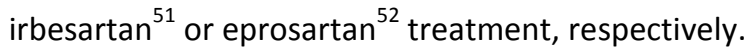

\section{THE GLYCATION PATHWAY AND CARDIOVASCULAR DISEASE}

Chapter 5 provided reference intervals for common carotid intima-media thickness (CIMT), based on a healthy sub-sample of combined subject-level data on common CIMT, as obtained by echotracking systems, from 24 different study centres worldwide. The additional assessment of the relation of cardiovascular risk factors with CIMT Z-scores enables future comparison of CIMT values across (patient) groups with different cardiovascular risk profiles, helping interpretation of such measures obtained both in research and clinical settings.

In Chapter 6, we hypothesized that genetic variation in the gene coding for glyoxalase 1 (GLO1; the rate-limiting enzyme in the detoxification of the major AGE precursors methylglyoxal and glyoxal ${ }^{71-78}$ ) may alter the expression and/or activity of GLO1 and may as such affect the genetic susceptibility to CVD. Indeed, the only known single nucleotide 
polymorphism (SNP) in the GLO1 gene existing within the coding sequence (i.e. a C/A substitution causing an Ala111Glu change) ${ }^{79}$ has been found to influence enzyme activity so that the presence of the A-allele results in reduced GLO1 activity. ${ }^{80}$ In addition, previous studies have shown positive associations of the presence of the $A$ allele with prevalent CVD and peripheral vascular disease ${ }^{81}$ and with overall mortality in haemodialysis patients, ${ }^{82}$ whereas no associations had been found of this GLO1 SNP with CIMT and plaques ${ }^{83}$ or with prevalent nephropathy and retinopathy ${ }^{84}$ in individuals with T2DM. The results in Chapter 6 were in line with these latter results, showing no (consistent) associations of nine common GLO1 gene SNPs (including Ala111Glu and which covered total common genetic variability of GLO1) with the prevalence of hypertension, markers of atherosclerosis [i.e. CIMT and ankle-brachial index $(\mathrm{ABI})$ ], arterial stiffness or renal function in two Dutch cohorts of individuals with normal glucose metabolism (NGM), impaired glucose metabolism (IGM) or T2DM (i.e. the Hoorn and CODAM studies).

Similarly, genetic variation in the gene coding for the receptor for AGEs [RAGE (i.e. $A G E R)$ ], has been shown to alter the expression, activity and/or ligand binding affinity of RAGE. $^{85,86}$ Specifically, the only known SNP existing within the coding sequence (i.e. Gly82Ser), has been found to influence RAGE binding affinity ${ }^{85}$ and the common SNPs 429T/C and -374T/A (in the RAGE promotor region) have been found to influence expression so that the presence of the $C$ and $A$ alleles, respectively, results in increased RAGE expression. ${ }^{86}$ These RAGE SNPs may as such affect the genetic susceptibility to CVD, though studies so far investigating the associations between RAGE SNPs and vascular disease have reported contradictory results, which may have been due to, amongst others, differences in individuals' glucose metabolism status (i.e. type 1 diabetes, T2DM or NGM; for details see Table $7.1^{86-110}$ ). The modifying effect of glucose metabolism status is supported by our current findings (Chapter 7) showing a protective role in terms of blood pressure and arterial stiffness of the AA genotype of SNP -374T/A in individuals with NGM, but a deleterious effect in individuals with IGM or T2DM. Although further studies are required to explain this effect, it might be that the nuclear binding factor, of which binding may be prevented by the $\mathrm{T}>\mathrm{A}$ substitution at position -374 , thereby influencing repression of RAGE transcription, ${ }^{86,111}$ is glucose-dependent.

To investigate the role of the glycation pathway in CVD, we finally measured plasma levels of both protein-bound (CML, CEL and pentosidine) and free [CML, CEL and 5-hydro5-methylimidazolone (MG-H1)] AGEs in individuals with NGM, IGM or T2DM from the Hoorn and CODAM studies. Chapter 8 showed that, overall, none of the protein-bound or free AGEs were independently associated with prevalent CVD, common CIMT or ABI, with the exception of free levels of $C M L$, which were positively associated with $A B I$ [Standardized $\beta=0.106$ SD (95\% Cl: 0.064 to 0.148$)$ ]. A general appraisal of the literature on the associations between plasma AGEs and (surrogate markers of) CVD in individuals with and without T2DM shows inconsistent findings, i.e. both positive ${ }^{112-117}$ and lack of 
associations $^{118-120}$ have been described. The lack of independent associations, after adjustment for potential confounding factors, between plasma AGEs and CVD and surrogate markers thereof in Chapter 8 are thus consistent with some of the previous studies. ${ }^{118-120}$ The inconsistencies with other studies may have been due to inconsistent or incomplete consideration of potential confounding factors such as age and/or GFR. ${ }^{112,114 \text {, }}$ 118,119

\section{METHODOLOGICAL CONSIDERATIONS}

\section{STUDY POPULATIONS}

In Chapter 3 and 4 we performed post-hoc analyses on two randomized trials. ${ }^{70,121}$

The first (Chapter 3) was a single-centre (Steno Diabetes Center, Gentofte, Denmark), prospective, randomized, double-blind, double-dummy, crossover trial of metformin vs. repaglinide with 96 non-obese $\left(\mathrm{BMI} \leq 27 \mathrm{~kg} / \mathrm{m}^{2}\right.$ ) insulin-naive individuals with T2DM. ${ }^{121}$ Patients were randomised to receive either $2 \mathrm{mg}$ repaglinide three times daily plus placebo metformin followed by $1 \mathrm{~g}$ metformin twice daily plus placebo repaglinide, or vice versa, each treatment for a period of four months with a one-month washout period (on diet-only) between interventions. The primary endpoint was $\mathrm{HbAlc}^{121}$ and the study in Chapter 3 focused specifically on the effects of treatments on blood levels of 3DG, which due to the complexity of its assessment only became available recently. The 'apparent' post-hoc nature of this study might be a point of concern, but in fact, this might not be considered a post-hoc study in its true sense. The 3DG measurements were only possible after blood collection in specific tubes and, therefore, prior to initiation of this trial, we sent these tubes to the Steno Diabetes Center to enable appropriate collection of blood for these 3DG measurements. The presence of carry-over effects in a trial with a crossover design could constitute another limitation of this study although the two treatment sequence groups were well balanced (Table 3.1 and 3.2). In addition, we attempted to adjust for possible carry-over effects to the extent these may have influenced the effects of period and treatment sequence. Finally, this trial was carried out with non-obese patients with T2DM. The extent to which our findings may be extrapolated to obese patients thus remains uncertain and should be further evaluated.

The second (Chapter 4) was a randomized, double-blind, placebo-controlled trial (conducted in 96 centres worldwide) of irbesartan with 590 hypertensive patients with T2DM and persistent microalbuminuria. ${ }^{70}$ Patients were randomly assigned to receive 150 mg irbesartan once daily, $300 \mathrm{mg}$ irbesartan once daily, or matching placebo for a median of two years. The primary endpoint was the development of overt nephropathy and the 
study in Chapter 4 specifically focused on the effect of irbesartan treatment on plasma levels of the AGEs CML and CEL, which were determined at baseline and at 1- and 2-years follow-up, in samples from 139 patients in the treatment arm receiving 300 mg irbesartan and from 125 patients receiving placebo, because, unfortunately, all samples from the 150 mg irbesartan group were discarded by error. However, in the initial analyses of this study, no significant effects of $150 \mathrm{mg}$ irbesartan treatment versus placebo were found on the development of nephropathy, creatinine clearance or blood pressure. ${ }^{70}$ Another possible limitation lies in the post-hoc nature of this study. However, given its design and size, it provides the best evidence so far with this regard.

In the Reference Values for Arterial Measurements' IMT database (Chapter 5), we combined subject-level data on established cardiovascular risk factors and common CIMT as obtained by echotracking systems from 24 different research centres - corresponding to 30 distinct cohorts - distributed across 14 countries worldwide. A total of 24,871 individuals with data available on common CIMT, age [range 15-101 years], sex (13,290 men/11,581 women), CVD status, and important cardiovascular risk factors were finally included in the analyses. To generate age- and sex-specific normative tables for common CIMT, we selected a healthy sub-population $(n=4,234)$ composed of individuals without a history of CVD, who did not use blood pressure-, lipid- and/or glucose-lowering medication and who had no (other) established cardiovascular risk factors (i.e. hypertension, smoking, diabetes, dyslipidaemia and/or obesity). The validity (and precision) of risk factor measurements depended on available data within the original studies included, which therefore differed between studies, and may have influenced the CIMT normative value estimates. Similarly, differences in CIMT measurement techniques were standardised between studies also by taking into account other factors potentially influencing common CIMT and differing between centres. However, the calibration may still have been sub-optimal in the case we have missed differences (e.g. in characteristics and/or measurement techniques) between studies, which might have influenced the results. Nevertheless, these differences also increase the heterogeneity of the study population and thereby the external validity of the results.

In Chapters 6-8, we combined data of participants from the follow-up examination of the Hoorn study ${ }^{122}$ and the baseline examination of the Cohort on Diabetes and Atherosclerosis Maastricht (CODAM) study. ${ }^{123,}{ }^{124}$ Both studies are ongoing prospective cohort studies designed to investigate glucose metabolism and CVD risk factors. For the follow-up examination of the Hoorn study (2000-2001) - which originally constituted a sample (aged 50-75 years) of the general population of Hoorn, the Netherlands individuals with IGM and T2DM were over-sampled, and 188 individuals with T2DM from the Hoorn Screening study were added. ${ }^{122,125}$ For the CODAM study, between 1999 and 2001 , individuals (aged $>40$ years) were selected from a large population-based cohort on the basis of an elevated risk for T2DM and/or CVD. ${ }^{124}$ Both cohorts thus included cross- 
sectional data on middle-aged Caucasian individuals at high risk for (or with prevalent) T2DM and/or CVD. As a result, the findings in Chapters 6-8 may not apply to the general (or younger) Caucasian population or to other ethnicities. In addition, given the crosssectional design, we cannot completely rule out the possibility that the lack of associations of plasma AGEs with prevalent CVD in particular (Chapter 8) might have resulted from possible post-event lifestyle and/or treatment changes that potentially influenced plasma AGEs and thereby may have masked the 'effects'. Similarly, we cannot rule out the lack of involvement of plasma AGEs in incident CVD and/or in other populations or disease states. Indeed, higher levels of protein-bound AGEs as examined herein were associated with higher risk for incident CVD and all-cause mortality among individuals with type 1 diabetes. $^{126}$

\section{MEASUREMENTS}

\section{MEASUREMENTS OF THE GLYCATION PATHWAY}

Circulating levels of AGEs probably constitute the most commonly used measurements of the glycation pathway, although, at present, a range of (other) glycation-related measures is available. Also for the studies in this thesis (Chapters 3, 4 and 8), we measured plasma levels of AGEs as markers of the glycation pathway. We quantitatively determined specific AGE epitopes by using specific analytical techniques such as high-performance liquid chromatography (HPLC; to determine pentosidine) and ultra performance liquid chromatography tandem mass spectrometry (UPLC-MS/MS; to determine CML, CEL, MG$\mathrm{H} 1$ and pyrraline), which are, at present, considered to be most accurate. ${ }^{127}$ However, others have also used immunoassays to determine plasma AGEs in observational ${ }^{112,114-120 \text {, }}$

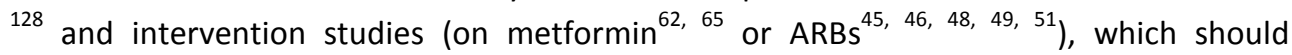
therefore be interpreted with caution as these immunoassays only yield semi-quantitative results. Furthermore, the differences in techniques used, may, in part, explain the contrasting results between our and (some) previous studies. More importantly, it should be emphasised, that it is currently uncertain whether elevated circulating AGE levels (either determined with HPLC, UPLC-MS/MS or immunoassays) adequately reflect the burden of the glycation pathway in the organs/tissues of individuals with or without T2DM. Specifically, the production and accumulation of AGEs is increased under hyperglycaemic conditions, ${ }^{74,76,129,130}$ whereas elevated plasma AGE levels in individuals with IGM and T2DM vs. those with NGM have not consistently been found (Chapter $8^{113 \text {, }}$ $116,120,128,131-133$ ), and even decreased levels of plasma CML with deteriorating glucose metabolism, as we also described in Chapter 8 , have been reported. ${ }^{134}$ In addition, previous studies have shown AGE accumulation in heart tissue of acute myocardial 
infarction patients, ${ }^{135}$ human vessel walls and atherosclerotic lesions, ${ }^{136-138}$ which was increased in individuals with diabetes, ${ }^{136,137,139}$ whereas epidemiological evidence of an adverse association between increased plasma levels of AGEs and CVD in individuals with or without T2DM remains uncertain, i.e. both positive ${ }^{112-117}$ and lack of associations ${ }^{118-120}$ have been described. Importantly, we therefore cannot fully discard the possibility that the discrepancy between the (negative) results from clinical intervention studies, as summarized in Chapter 2 and investigated in Chapters 3 and 4, and the beneficial effects of various therapies shown in in vitro and animal experiments, lies in the type of tissue examined. Similarly, the lack of adverse associations of plasma AGEs, as markers of the glycation pathway, with CVD in the epidemiological study in Chapter 8 may have resulted from the potentially sub-optimal reflection of the tissue glycation burden by the plasma AGEs measured. However, additional (ideally large and prospective) cohort studies are warranted to fully elucidate the role of plasma AGEs in (T2DM-related) CVD.

Different measurements of the glycation pathway, such as blood levels of dicarbonyl compounds, may better reflect the burden of the glycation pathway in tissues and/or the glycation processes that may be affected by treatment. Indeed, Chapter 3 shows that both metformin and repaglinide treatment lowered blood levels of the dicarbonyl 3DG, whereas plasma levels of the AGEs CML and CEL did not change significantly during either treatment. ${ }^{64}$ Alternatively, (invasive) measures of tissue AGE accumulation in the skin ${ }^{140,}$ ${ }^{141}$ have been linked with the presence and severity of diabetic complications and have been shown to decrease after intensified anti-hyperglycaemic treatment. ${ }^{142}$ Interestingly, non-invasively measured skin auto-fluorescence, which has been shown to correlate well with the AGE content in skin biopsies, ${ }^{143,}{ }^{144}$ has been consistently associated with diabetic micro- and macrovascular complications ${ }^{145}$ and may thus represent another useful measurement of the glycation pathway.

\section{MEASUREMENTS OF CARDIOVASCULAR DISEASE}

To investigate the role of the glycation pathway in CVD (in Part 2), we investigated, in the Hoorn and CODAM studies, associations of measures of the glycation pathway with CVD and surrogate markers thereof. The main reason for the use of surrogate markers over (cardiovascular) morbidity and mortality as outcome variables in trials and/or (prospective) observational studies is the considerable reduction in sample size and possibly in duration of follow-up necessary. In addition, the development of cardiovascular events is a heterogeneous process and measurement of surrogate markers of distinct potentially underlying pathophysiological mechanisms (e.g. atherosclerosis and/or arterial stiffness) enables the study of pathways (e.g. glycation) involved in these specific and distinct mechanisms. 
Markers of atherosclerosis used in this thesis were CIMT and ABI. CIMT, as obtained with the use of non-invasive ultrasound imaging, ${ }^{146}$ is regarded a suitable surrogate marker for $\mathrm{CVD}^{147}$ and is currently widely used in clinical and epidemiological studies. In addition, Chapter 5 provided age- and sex-specific percentiles of common CIMT in a healthy population and the relation of cardiovascular risk factors with CIMT Z-scores, which enables comparison of CIMT values for (patient) groups with different cardiovascular risk profiles. These are particularly useful for the interpretation of CIMT measures obtained both in research and clinical settings. $\mathrm{ABI}$, i.e., the ratio of the ankle systolic pressure to the brachial systolic pressure, is currently widely used to evaluate patients with peripheral artery disease, which is considered to be present whenever $A B I<0.9 .{ }^{148,149}$ Although both low (usually set at $<0.9$ ) and high (usually set at $>1.4$ ) $A B I$ are associated with increased risk for incident CVD, ${ }^{149}$ in this thesis individuals who had an $A B I>1.4(n=7)$ were excluded from the analyses as this is indicative of arterial calcification ${ }^{150}$ rather than peripheral artery disease. Consequently, when using $A B I$ as a continuous variable, which increased the statistical power of our analyses, lower values indicated higher risk.

Markers of arterial stiffness used in this thesis were measures of (local) carotid, femoral and brachial artery stiffness, as obtained with the use of non-invasive ultrasound imaging. ${ }^{122,146}$ Increased carotid stiffness in particular has been associated with incident cardiovascular events in individuals with different cardiovascular risk profiles, ${ }^{151-154}$ but higher stiffness levels of peripheral arteries, such as the femoral and brachial artery, may also be detrimental to cardiovascular health. However, the exact interpretation of these local arterial stiffness measures has been hampered by the absence of reference values. Similar to Chapter 5, research into reference intervals for carotid stiffness is currently ongoing and aims to also improve interpretation of these measurements as obtained in both research and clinical settings.

\section{CLINICAL RELEVANCE}

Diabetes currently affects around 346 million people worldwide, ${ }^{155,156}$ a number that is expected to rise to 552 million by $2030 .^{157}$ As adults with diabetes experience a 2 -fold increased risk for cardiovascular events ${ }^{158}$ and cardiovascular death ${ }^{159}$ relative to those without diabetes, research into potential mechanisms explaining this increased risk is of importance. Specifically, extended knowledge into pathways leading to diabetic complications may provide possibilities for (novel) therapies to decrease the burden of this disease. In addition, identification of (novel) markers may be useful to identify individuals at high risk for diabetes and/or CVD. Experimental studies have extensively shown the importance of the glycation pathway in (diabetes-related) CVD as AGE 
accumulation in tissues has been linked to the development of vascular complications ${ }^{1,77,}$ 78 and (specific) AGE-inhibiting interventions have been shown to reduce vascular complications. $^{2-22,28-32}$ In this thesis we aimed to investigate the role of the glycation pathway in (T2DM-related) CVD and possibilities for AGE- inhibiting interventions in a human setting.

Although we found some evidence of 3DG-lowering effects of metformin, which had been proposed to hold dicarbonyl-scavenging potentials similar to aminoguanidine due to their similarities in structure, we found a similar reduction in 3DG after repaglinide, an anti-hyperglycaemic agent with a different working mechanism. However, the improved glycaemic control induced by metformin and repaglinide was thus associated with a (similar) reduction in 3DG levels, which was in turn associated with a reduction in endothelial markers and may as such constitute a metabolic pathway through which both treatments have a beneficial impact on the cardiovascular risk profile. In addition, we found no evidence of AGE-inhibiting effects (i.e. on plasma AGEs) of treatment with irbesartan and we found no (adverse) associations of plasma AGEs with CVD or markers thereof. However, it should be emphasised that it is currently uncertain whether elevated plasma AGEs adequately reflect the burden of the glycation pathway in the tissues of individuals with or without T2DM. The research presented in this thesis thus does not (necessarily) support the lack of a role of the glycation pathway in T2DM-related CVD in humans, but may rather indicate the importance of identification of (other) valid markers that (better) reflect the burden of the glycation pathway in the tissues. Such (noninvasive) markers of the glycation pathway may then, in addition to their value in epidemiological studies such as presented in this thesis, be potentially useful tools in the identification of individuals at high risk for developing diabetes and/or CVD.

CIMT measurements have by now been proposed for suchlike screening for cardiovascular risk as well as for fine tuning of individuals' risk prediction as ascertained by current risk algorithms such as the Framingham ${ }^{160}$ and SCORE. ${ }^{161}$ Although in some studies CIMT has not improved the discriminative value of these risk scores ${ }^{162-164}$ or did not lead to significant reclassification ${ }^{163,164}$ when used additionally to traditional cardiovascular risk factors, some studies have indeed shown that CIMT may have additional value in terms of significant reclassification, particularly among those at intermediate risk. ${ }^{165,166}$ Further and ongoing (IMPROVE, USE-IMT) studies will determine the added value, if any, of common CIMT measurements in individuals' risk stratification. For that purpose, reference intervals as presented in Chapter 5 may be helpful. Furthermore, reference intervals are in particular of help in clinical practice, where one is accustomed to use information that can be referred to as being normal, too low or too high. Chapter 5 provides such a reference to which patients' CIMT information can be related relative to what is expected to be 'normal'. 


\section{IMPLICATIONS FOR FUTURE RESEARCH}

This thesis evaluated 1 ) possible interventions in the glycation pathway and 2) the role of the glycation pathway in the aetiology of (T2DM-related) CVD in a number of epidemiological studies.

Although experimental studies concerning the specific AGE inhibitor aminoguanidine and AGE breaker alagebrium clearly resulted in beneficial effects, it seems that safety and/or efficacy in clinical studies with these compounds are of concern. With regard to the other therapies developed for purposes unrelated to glycation such as metformin and irbesartan, the current clinical evidence for (specific) AGE inhibition is fairly limited as study designs and/or measurement techniques have often been sub-optimal. In addition, it remains possible that the potential beneficial effects of the different therapies on accumulation or burden of AGEs (in tissues) may not translate to changes in plasma levels, which have often been assessed in trials as markers of the glycation pathway. More large, specific and well-designed studies are needed to fully elucidate the exact clinical impact of AGE inhibition and future studies should determine which (glycation-related) outcome measures reflect the glycation pathway most accurately and are most suitable for intervention studies.

Evidence for the role of the glycation pathway in (T2DM-related) CVD in epidemiological studies remains incomplete, although our studies have added some evidence for a role of decreasing 3DG levels in diabetes-related pathophysiological mechanisms (i.e. endothelial dysfunction). The associations between plasma AGEs and CVD have often been evaluated in rather small or specific patient populations. In addition, these studies have often been cross-sectional and/or confounding factors have not been consistently taken into consideration. Moreover, similar to the intervention studies described above, our findings (together with inconsistent findings in the literature so far) of no associations of plasma AGEs with CVD and surrogate markers thereof suggest that plasma levels of AGEs may not represent the burden of the glycation pathway (in tissues) accurately. Future research, ideally in large and prospective cohort studies should fully elucidate the role, if any, of plasma AGEs in (T2DM-related) CVD. In addition, future research aiming at developing and/or evaluating (novel) other markers of the glycation pathway [e.g. free methylglyoxal, AGE concentrations in circulating cells or tissue AGE accumulation (e.g. by skin autofluorescence)] would facilitate expanding the current epidemiological knowledge regarding the role of the glycation pathway in CVD. 


\section{REFERENCES}

1. Brownlee M. Biochemistry and molecular cell biology of diabetic complications. Nature. 2001;414:813-820

2. Thornalley PJ. Use of aminoguanidine (pimagedine) to prevent the formation of advanced glycation endproducts. Arch Biochem Biophys. 2003;419:31-40

3. Brownlee $M$, Vlassara $H$, Kooney A, Ulrich $P$, Cerami A. Aminoguanidine prevents diabetes-induced arterial wall protein cross-linking. Science. 1986;232:1629-1632

4. Fu MX, Wells-Knecht KJ, Blackledge JA, Lyons TJ, Thorpe SR, Baynes JW. Glycation, glycoxidation, and crosslinking of collagen by glucose. Kinetics, mechanisms, and inhibition of late stages of the maillard reaction. Diabetes. 1994;43:676-683

5. Huijberts MS, Wolffenbuttel BH, Boudier HA, Crijns FR, Kruseman AC, Poitevin P, et al. Aminoguanidine treatment increases elasticity and decreases fluid filtration of large arteries from diabetic rats. $J$ Clin Invest. 1993;92:1407-1411

6. Birrell AM, Heffernan SJ, Kirwan P, McLennan S, Gillin AG, Yue DK. The effects of aminoguanidine on renal changes in a baboon model of type 1 diabetes. J Diabetes Complications. 2002;16:301-309

7. Edelstein D, Brownlee M. Aminoguanidine ameliorates albuminuria in diabetic hypertensive rats. Diabetologia. 1992;35:96-97

8. Yamauchi A, Takei I, Makita Z, Nakamoto S, Ohashi N, Kiguchi H, et al. Effects of aminoguanidine on serum advanced glycation endproducts, urinary albilmin excretion, mesangial expansion, and glomerular basement membrane thickening in otsuka long-evans tokushima fatty rats. Diabetes Res Clin Pract. 1997;34:127-133

9. Soulis-Liparota T, Cooper M, Papazoglou D, Clarke B, Jerums G. Retardation by aminoguanidine of development of albuminuria, mesangial expansion, and tissue fluorescence in streptozocin-induced diabetic rat. Diabetes. 1991;40:1328-1334

10. Hammes HP, Martin S, Federlin K, Geisen K, Brownlee M. Aminoguanidine treatment inhibits the development of experimental diabetic retinopathy. Proc Natl Acad Sci U S A. 1991;88:11555-11558

11. Kern TS, Engerman RL. Pharmacological inhibition of diabetic retinopathy: Aminoguanidine and aspirin. Diabetes. 2001;50:1636-1642

12. Miyauchi Y, Shikama H, Takasu T, Okamiya H, Umeda M, Hirasaki E, et al. Slowing of peripheral motor nerve conduction was ameliorated by aminoguanidine in streptozocin-induced diabetic rats. Eur J Endocrinol. 1996;134:467-473

13. Schmidt RE, Dorsey DA, Beaudet LN, Reiser KM, Williamson JR, Tilton RG. Effect of aminoguanidine on the frequency of neuroaxonal dystrophy in the superior mesenteric sympathetic autonomic ganglia of rats with streptozocin-induced diabetes. Diabetes. 1996;45:284-290

14. Asif M, Egan J, Vasan S, Jyothirmayi GN, Masurekar MR, Lopez S, et al. An advanced glycation endproduct cross-link breaker can reverse age-related increases in myocardial stiffness. Proc Natl Acad Sci U S A. 2000;97:2809-2813

15. Candido R, Forbes JM, Thomas MC, Thallas V, Dean RG, Burns WC, et al. A breaker of advanced glycation end products attenuates diabetes-induced myocardial structural changes. Circ Res. 2003;92:785-792

16. Vaitkevicius PV, Lane M, Spurgeon H, Ingram DK, Roth GS, Egan JJ, et al. A cross-link breaker has sustained effects on arterial and ventricular properties in older rhesus monkeys. Proc Natl Acad Sci U S A. 2001;98:1171-1175

17. Vasan S, Zhang X, Zhang X, Kapurniotu A, Bernhagen J, Teichberg S, et al. An agent cleaving glucose-derived protein crosslinks in vitro and in vivo. Nature. 1996;382:275-278

18. Wolffenbuttel BH, Boulanger CM, Crijns FR, Huijberts MS, Poitevin P, Swennen GN, et al. Breakers of advanced glycation end products restore large artery properties in experimental diabetes. Proc Natl Acad Sci U S A. 1998;95:4630-4634

19. Forbes JM, Yee LT, Thallas V, Lassila M, Candido R, Jandeleit-Dahm KA, et al. Advanced glycation end product interventions reduce diabetes-accelerated atherosclerosis. Diabetes. 2004;53:1813-1823

20. Forbes JM, Thallas V, Thomas MC, Founds HW, Burns WC, Jerums G, et al. The breakdown of preexisting advanced glycation end products is associated with reduced renal fibrosis in experimental diabetes. Faseb $\mathrm{J}$. 2003;17:1762-1764 
21. Lassila M, Seah KK, Allen TJ, Thallas V, Thomas MC, Candido R, et al. Accelerated nephropathy in diabetic apolipoprotein e-knockout mouse: Role of advanced glycation end products. J Am Soc Nephrol. 2004;15:2125-2138

22. Thallas-Bonke V, Lindschau C, Rizkalla B, Bach LA, Boner G, Meier M, et al. Attenuation of extracellular matrix accumulation in diabetic nephropathy by the advanced glycation end product cross-link breaker alt711 via a protein kinase c-alpha-dependent pathway. Diabetes. 2004;53:2921-2930

23. Bolton WK, Cattran DC, Williams ME, Adler SG, Appel GB, Cartwright K, et al. Randomized trial of an inhibitor of formation of advanced glycation end products in diabetic nephropathy. Am $J$ Nephrol. 2004;24:32-40

24. Freedman BI, Wuerth JP, Cartwright K, Bain RP, Dippe S, Hershon K, et al. Design and baseline characteristics for the aminoguanidine clinical trial in overt type 2 diabetic nephropathy (action ii). Control Clin Trials. 1999;20:493-510

25. Kass DA, Shapiro EP, Kawaguchi M, Capriotti AR, Scuteri A, deGroof RC, et al. Improved arterial compliance by a novel advanced glycation end-product crosslink breaker. Circulation. 2001;104:1464-1470

26. Hartog JW, Willemsen S, van Veldhuisen DJ, Posma JL, van Wijk LM, Hummel YM, et al. Effects of alagebrium, an advanced glycation endproduct breaker, on exercise tolerance and cardiac function in patients with chronic heart failure. Eur J Heart Fail. 2011;13:899-908

27. ClinicalTrials.gov. Registry and results database of federally and privately supported clinical trials conducted in the united states and around the world. 2012;Accessed February 2012

28. Booth AA, Khalifah RG, Hudson BG. Thiamine pyrophosphate and pyridoxamine inhibit the formation of antigenic advanced glycation end-products: Comparison with aminoguanidine. Biochem Biophys Res Commun. 1996;220:113-119

29. Booth AA, Khalifah RG, Todd P, Hudson BG. In vitro kinetic studies of formation of antigenic advanced glycation end products (ages). Novel inhibition of post-amadori glycation pathways. $J$ Biol Chem. 1997;272:5430-5437

30. Voziyan PA, Khalifah RG, Thibaudeau C, Yildiz A, Jacob J, Serianni AS, et al. Modification of proteins in vitro by physiological levels of glucose: Pyridoxamine inhibits conversion of amadori intermediate to advanced glycation end-products through binding of redox metal ions. J Biol Chem. 2003;278:46616-46624

31. Degenhardt TP, Alderson NL, Arrington DD, Beattie RJ, Basgen JM, Steffes MW, et al. Pyridoxamine inhibits early renal disease and dyslipidemia in the streptozotocin-diabetic rat. Kidney Int. 2002;61:939-950

32. Stitt A, Gardiner TA, Alderson NL, Canning P, Frizzell N, Duffy N, et al. The age inhibitor pyridoxamine inhibits development of retinopathy in experimental diabetes. Diabetes. 2002;51:2826-2832

33. Alkhalaf A, Klooster A, van Oeveren W, Achenbach U, Kleefstra N, Slingerland RJ, et al. A double-blind, randomized, placebo-controlled clinical trial on benfotiamine treatment in patients with diabetic nephropathy. Diabetes Care. 2010;33:1598-1601

34. Hammes HP, Du X, Edelstein D, Taguchi T, Matsumura T, Ju Q, et al. Benfotiamine blocks three major pathways of hyperglycemic damage and prevents experimental diabetic retinopathy. Nat Med. 2003;9:294299

35. Babaei-Jadidi R, Karachalias N, Ahmed N, Battah S, Thornalley PJ. Prevention of incipient diabetic nephropathy by high-dose thiamine and benfotiamine. Diabetes. 2003;52:2110-2120

36. Balakumar P, Chakkarwar VA, Singh M. Ameliorative effect of combination of benfotiamine and fenofibrate in diabetes-induced vascular endothelial dysfunction and nephropathy in the rat. Mol Cell Biochem. 2009;320:149-162

37. Ruggiero-Lopez D, Lecomte M, Moinet G, Patereau G, Lagarde M, Wiernsperger N. Reaction of metformin with dicarbonyl compounds. Possible implication in the inhibition of advanced glycation end product formation. Biochem Pharmacol. 1999;58:1765-1773

38. Miyata T, van Ypersele de Strihou C, Ueda Y, Ichimori K, Inagi R, Onogi $H$, et al. Angiotensin ii receptor antagonists and angiotensin-converting enzyme inhibitors lower in vitro the formation of advanced glycation end products: Biochemical mechanisms. J Am Soc Nephrol. 2002;13:2478-2487

39. Forbes JM, Thomas MC, Thorpe SR, Alderson NL, Cooper ME. The effects of valsartan on the accumulation of circulating and renal advanced glycation end products in experimental diabetes. Kidney Int Suppl. 2004:S105-107

40. Nangaku M, Miyata T, Sada T, Mizuno M, Inagi R, Ueda Y, et al. Anti-hypertensive agents inhibit in vivo the formation of advanced glycation end products and improve renal damage in a type 2 diabetic nephropathy rat model. J Am Soc Nephrol. 2003;14:1212-1222 
41. Fan Q, Liao J, Kobayashi M, Yamashita M, Gu L, Gohda T, et al. Candesartan reduced advanced glycation end-products accumulation and diminished nitro-oxidative stress in type 2 diabetic kk/ta mice. Nephrol Dial Transplant. 2004;19:3012-3020

42. Izuhara $Y$, Sada T, Yanagisawa H, Koike H, Ohtomo S, Dan T, et al. A novel sartan derivative with very low angiotensin ii type 1 receptor affinity protects the kidney in type 2 diabetic rats. Arterioscler Thromb Vasc Biol. 2008;28:1767-1773

43. Sugiyama T, Okuno T, Fukuhara M, Oku H, Ikeda T, Obayashi H, et al. Angiotensin ii receptor blocker inhibits abnormal accumulation of advanced glycation end products and retinal damage in a rat model of type 2 diabetes. Exp Eye Res. 2007;85:406-412

44. Thomas MC, Tikellis C, Burns WM, Bialkowski K, Cao Z, Coughlan MT, et al. Interactions between renin angiotensin system and advanced glycation in the kidney. J Am Soc Nephrol. 2005;16:2976-2984

45. Komiya N, Hirose H, Saisho Y, Saito I, Itoh H. Effects of 12-month valsartan therapy on glycation and oxidative stress markers in type 2 diabetic subjects with hypertension. Int Heart J. 2008;49:681-689

46. Mitsuhashi H, Tamura K, Yamauchi J, Ozawa M, Yanagi M, Dejima T, et al. Effect of losartan on ambulatory short-term blood pressure variability and cardiovascular remodeling in hypertensive patients on hemodialysis. Atherosclerosis. 2009;207:186-190

47. Rabbani N, Adaikalakoteswari A, Rossing K, Rossing P, Tarnow L, Parving HH, et al. Effect of irbesartan treatment on plasma and urinary markers of protein damage in patients with type 2 diabetes and microalbuminuria. Amino Acids. 2011

48. Saisho $\mathrm{Y}$, Komiya N, Hirose H. Effect of valsartan, an angiotensin ii receptor blocker, on markers of oxidation and glycation in japanese type 2 diabetic subjects: Blood pressure-independent effect of valsartan. Diabetes Res Clin Pract. 2006;74:201-203

49. Monacelli F, Poggi A, Storace D, Durante A, Traverso N, Viviani GL, et al. Effects of valsartan therapy on protein glycoxidation. Metabolism. 2006;55:1619-1624

50. Saha SA, LaSalle BK, Clifton GD, Short RA, Tuttle KR. Modulation of advanced glycation end products by candesartan in patients with diabetic kidney disease--a dose-response relationship study. Am J Ther. 2010;17:553-558

51. Busch M, Franke S, Wolf G, Rohde RD, Stein G. Serum levels of the advanced glycation end products nepsilon-carboxymethyllysine and pentosidine are not influenced by treatment with the angiotensin receptor ii type 1 blocker irbesartan in patients with type 2 diabetic nephropathy and hypertension. Nephron Clin Pract. 2008;108:c291-297

52. Hartog JW, van de Wal RM, Schalkwijk CG, Miyata T, Jaarsma W, Plokker HW, et al. Advanced glycation endproducts, anti-hypertensive treatment and diastolic function in patients with hypertension and diastolic dysfunction. Eur J Heart Fail. 2010

53. Williams ME, Bolton WK, Khalifah RG, Degenhardt TP, Schotzinger RJ, McGill JB. Effects of pyridoxamine in combined phase 2 studies of patients with type 1 and type 2 diabetes and overt nephropathy. Am J Nephrol. 2007;27:605-614

54. Lewis EJ, Greene T, Spitalewiz S, Blumenthal S, Berl T, Hunsicker LG, et al. Pyridorin in type 2 diabetic nephropathy. J Am Soc Nephrol. 2011

55. Nascimento MM, Suliman ME, Murayama Y, Nihi M, Hayashi SY, Stenvinkel P, et al. Effect of high-dose thiamine and pyridoxine on advanced glycation end products and other oxidative stress markers in hemodialysis patients: A randomized placebo-controlled study. J Ren Nutr. 2006;16:119-124

56. Schupp N, Dette EM, Schmid U, Bahner U, Winkler M, Heidland A, et al. Benfotiamine reduces genomic damage in peripheral lymphocytes of hemodialysis patients. Naunyn Schmiedebergs Arch Pharmacol. 2008;378:283-291

57. Stirban A, Negrean M, Stratmann B, Gawlowski T, Horstmann T, Gotting C, et al. Benfotiamine prevents macro- and microvascular endothelial dysfunction and oxidative stress following a meal rich in advanced glycation end products in individuals with type 2 diabetes. Diabetes Care. 2006;29:2064-2071

58. Du X, Edelstein D, Brownlee M. Oral benfotiamine plus alpha-lipoic acid normalises complication-causing pathways in type 1 diabetes. Diabetologia. 2008;51:1930-1932

59. Faure P, Wiernsperger N, Polge C, Favier A, Halimi S. Impairment of the antioxidant properties of serum albumin in patients with diabetes: Protective effects of metformin. Clin Sci (Lond). 2008;114:251-256

60. Beisswenger PJ, Howell SK, Touchette AD, Lal S, Szwergold BS. Metformin reduces systemic methylglyoxal levels in type 2 diabetes. Diabetes. 1999;48:198-202 
61. Rabbani N, Chittari MV, Bodmer CW, Zehnder D, Ceriello A, Thornalley PJ. Increased glycation and oxidative damage to apolipoprotein b100 of Idl cholesterol in patients with type 2 diabetes and effect of metformin. Diabetes. 2010;59:1038-1045

62. Diamanti-Kandarakis E, Alexandraki K, Piperi C, Aessopos A, Paterakis T, Katsikis I, et al. Effect of metformin administration on plasma advanced glycation end product levels in women with polycystic ovary syndrome. Metabolism. 2007; 56:129-134

63. Engelen L, Lund SS, Ferreira I, Tarnow L, Parving HH, Gram J, et al. Improved glycemic control induced by both metformin and repaglinide is associated with a reduction in blood levels of 3-deoxyglucosone in nonobese patients with type 2 diabetes. Eur J Endocrinol. 2011;164:371-379

64. Lund SS, Tarnow L, Stehouwer CD, Schalkwijk CG, Teerlink T, Gram J, et al. Impact of metformin versus repaglinide on non-glycaemic cardiovascular risk markers related to inflammation and endothelial dysfunction in non-obese patients with type 2 diabetes. Eur J Endocrinol. 2008;158:631-641

65. Kanazawa I, Yamamoto M, Yamaguchi T, Sugimoto T. Effects of metformin and pioglitazone on serum pentosidine levels in type 2 diabetes mellitus. Exp Clin Endocrinol Diabetes. 2011;119:362-365

66. Persson $F$, Rossing $\mathrm{P}$, Hovind $\mathrm{P}$, Stehouwer $\mathrm{CD}$, Schalkwijk $\mathrm{C}$, Tarnow $\mathrm{L}$, et al. Irbesartan treatment reduces biomarkers of inflammatory activity in patients with type 2 diabetes and microalbuminuria: An irma 2 substudy. Diabetes. 2006;55:3550-3555

67. Engelen L, Persson F, Ferreira I, Rossing P, Hovind P, Teerlink T, et al. Irbesartan treatment does not influence plasma levels of the advanced glycation end products $n$ (epsilon)(1-carboxymethyl)lysine and n(epsilon)(1-carboxyethyl)lysine in patients with type 2 diabetes and microalbuminuria. A randomized controlled trial. Nephrol Dial Transplant. 2011;26:3573-3577

68. UK Prospective Diabetes Study (UKPDS) Group. Effect of intensive blood-glucose control with metformin on complications in overweight patients with type 2 diabetes (ukpds 34). Uk prospective diabetes study (ukpds) group. Lancet. 1998;352:854-865

69. Brenner BM, Cooper ME, de Zeeuw D, Keane WF, Mitch WE, Parving HH, et al. Effects of losartan on renal and cardiovascular outcomes in patients with type 2 diabetes and nephropathy. $N$ Engl J Med. 2001;345:861-869

70. Parving HH, Lehnert $\mathrm{H}$, Brochner-Mortensen J, Gomis R, Andersen S, Arner P. The effect of irbesartan on the development of diabetic nephropathy in patients with type 2 diabetes. N Engl J Med. 2001;345:870-878

71. Thornalley PJ. Glyoxalase i--structure, function and a critical role in the enzymatic defence against glycation. Biochem Soc Trans. 2003;31:1343-1348

72. Rabbani N, Thornalley PJ. Glyoxalase in diabetes, obesity and related disorders. Semin Cell Dev Biol. 2011;22:309-317

73. Thornalley PJ. Glutathione-dependent detoxification of alpha-oxoaldehydes by the glyoxalase system: Involvement in disease mechanisms and antiproliferative activity of glyoxalase i inhibitors. Chem Biol Interact. 1998;111-112:137-151

74. Shinohara M, Thornalley PJ, Giardino I, Beisswenger P, Thorpe SR, Onorato J, et al. Overexpression of glyoxalase-i in bovine endothelial cells inhibits intracellular advanced glycation endproduct formation and prevents hyperglycemia-induced increases in macromolecular endocytosis. J Clin Invest. 1998;101:11421147

75. Kalapos MP. Methylglyoxal in living organisms: Chemistry, biochemistry, toxicology and biological implications. Toxicol Lett. 1999;110:145-175

76. Brouwers O, Niessen PM, Ferreira I, Miyata T, Scheffer PG, Teerlink T, et al. Overexpression of glyoxalase-i reduces hyperglycemia-induced levels of advanced glycation end products and oxidative stress in diabetic rats. J Biol Chem. 2011;286:1374-1380

77. Brouwers O, Niessen PMG, Miyata T, Østergaard J, Flyvbjerg A, Peutz-Kootstra CJ, et al. Glyoxalase-i overexpression attenuates early renal impairment in diabetic rats. Diabetologia. 2011;54:S44

78. Brouwers O, Niessen PMG, Miyata T, Teerlink T, Janssen BJ, De Mey JGR, et al. Overexpression of glyoxalase-i improves vascular function in a rat model of diabetes. Diabetologia. 2010;53:S520

79. Gale CP, Grant PJ. The characterisation and functional analysis of the human glyoxalase-1 gene using methods of bioinformatics. Gene. 2004;340:251-260

80. Barua M, Jenkins EC, Chen W, Kuizon S, Pullarkat RK, Junaid MA. Glyoxalase i polymorphism rs2736654 causing the ala111glu substitution modulates enzyme activity--implications for autism. Autism Res. 2011;4:262-270 
81. Kalousova M, Germanova A, Jachymova M, Mestek O, Tesav V, Zima T. A419c (e111a) polymorphism of the glyoxalase $\mathrm{i}$ gene and vascular complications in chronic hemodialysis patients. Ann N Y Acad Sci. 2008;1126:268-271

82. Kalousova M, Jachymova M, Germanova A, Kubena AA, Tesar V, Zima T. Genetic predisposition to advanced glycation end products toxicity is related to prognosis of chronic hemodialysis patients. Kidney Blood Press Res. 2010;33:30-36

83. Wu JC, Li XH, Wang JB, Tang JF, Wang YF, Peng YD. Glyoxalase i and aldose reductase gene polymorphisms and susceptibility to carotid atherosclerosis in type 2 diabetes. Genet Test Mol Biomarkers. 2011;15:273279

84. Wu JC, Li XH, Peng YD, Wang JB, Tang JF, Wang YF. Association of two glyoxalase i gene polymorphisms with nephropathy and retinopathy in type 2 diabetes. J Endocrinol Invest. 2011;34:e343-348

85. Hofmann MA, Drury S, Hudson BI, Gleason MR, Qu W, Lu Y, et al. Rage and arthritis: The g82s polymorphism amplifies the inflammatory response. Genes Immun. 2002;3:123-135

86. Hudson BI, Stickland MH, Futers TS, Grant PJ. Effects of novel polymorphisms in the rage gene on transcriptional regulation and their association with diabetic retinopathy. Diabetes. 2001;50:1505-1511

87. dos Santos KG, Canani LH, Gross JL, Tschiedel B, Pires Souto KE, Roisenberg I. The -374a allele of the receptor for advanced glycation end products gene is associated with a decreased risk of ischemic heart disease in african-brazilians with type 2 diabetes. Mol Genet Metab. 2005;85:149-156

88. Lindholm E, Bakhtadze E, Sjogren M, Cilio CM, Agardh E, Groop L, et al. The -374 t/a polymorphism in the gene encoding rage is associated with diabetic nephropathy and retinopathy in type 1 diabetic patients. Diabetologia. 2006;49:2745-2755

89. Pettersson-Fernholm K, Forsblom C, Hudson BI, Perola M, Grant PJ, Groop PH. The functional - 374 t/a rage gene polymorphism is associated with proteinuria and cardiovascular disease in type 1 diabetic patients. Diabetes. 2003;52:891-894

90. Picheth G, Costantini CO, Pedrosa FO, Leme da Rocha Martinez T, Maltempi de Souza E. The -374a allele of the receptor for advanced glycation end products (rage) gene promoter is a protective factor against cardiovascular lesions in type 2 diabetes mellitus patients. Clin Chem Lab Med. 2007;45:1268-1272

91. Poirier O, Nicaud V, Vionnet N, Raoux S, Tarnow L, Vlassara H, et al. Polymorphism screening of four genes encoding advanced glycation end-product putative receptors. Association study with nephropathy in type 1 diabetic patients. Diabetes. 2001;50:1214-1218

92. Shim CY, Park S, Yoon SJ, Park HY, Kim HT, Oh B, et al. Association of rage gene polymorphisms with in-stent restenosis in non-diabetic korean population. Cardiology. 2007;107:261-268

93. Yoon SJ, Park S, Shim CY, Park CM, Ko YG, Choi D, et al. Association of rage gene polymorphisms with coronary artery disease in the korean population. Coron Artery Dis. 2007;18:1-8

94. Zee RY, Romero JR, Gould JL, Ricupero DA, Ridker PM. Polymorphisms in the advanced glycosylation end product-specific receptor gene and risk of incident myocardial infarction or ischemic stroke. Stroke. 2006;37:1686-1690

95. Falcone C, Campo I, Emanuele E, Buzzi MP, Geroldi D, Belvito C, et al. -374t/a polymorphism of the rage gene promoter in relation to severity of coronary atherosclerosis. Clin Chim Acta. 2005;354:111-116

96. Falcone C, Geroldi D, Buzzi MP, Emanuele E, Yilmaz Y, Fontana JM, et al. The -374t/a rage polymorphism protects against future cardiac events in nondiabetic patients with coronary artery disease. Arch Med Res. 2008;39:320-325

97. Globocnik Petrovic M, Steblovnik K, Peterlin B, Petrovic D. The - 429 t/c and - 374 t/a gene polymorphisms of the receptor of advanced glycation end products gene are not risk factors for diabetic retinopathy in caucasians with type 2 diabetes. Klin Monatsbl Augenheilkd. 2003;220:873-876

98. Hofmann MA, Yang Q, Harja E, Kedia P, Gregersen PK, Cupples LA, et al. The rage gly82ser polymorphism is not associated with cardiovascular disease in the framingham offspring study. Atherosclerosis. 2005;182:301-305

99. JiXiong X, BiLin X, MingGong Y, ShuQin L. -429t/c and -374t/a polymorphisms of rage gene promoter are not associated with diabetic retinopathy in chinese patients with type 2 diabetes. Diabetes Care. 2003;26:26962697

100. Kirbis J, Milutinovic A, Steblovnik K, Teran N, Terzic R, Zorc M. The -429 t/c and -374 t/a gene polymorphisms of the receptor of advanced glycation end products gene (rage) are not risk factors for coronary artery disease in slovene population with type 2 diabetes. Coll Antropol. 2004;28:611-616

101. Lindholm E, Bakhtadze E, Cilio C, Agardh E, Groop L, Agardh CD. Association between Ita, tnf and ager polymorphisms and late diabetic complications. PLOS ONE. 2008;3:e2546 
102. Prevost G, Fajardy I, Besmond C, Balkau B, Tichet J, Fontaine P, et al. Polymorphisms of the receptor of advanced glycation endproducts (rage) and the development of nephropathy in type 1 diabetic patients. Diabetes Metab. 2005;31:35-39

103. Pulkkinen A, Viitanen L, Kareinen A, Lehto S, Laakso M. Gly82ser polymorphism of the receptor of advanced glycation end product gene is not associated with coronary heart disease in finnish nondiabetic subjects or in patients with type 2 diabetes. Diabetes Care. 2000;23:864

104. Ramprasad S, Radha V, Mathias RA, Majumder PP, Rao MR, Rema M. Rage gene promoter polymorphisms and diabetic retinopathy in a clinic-based population from south india. Eye. 2007;21:395-401

105. Yoshioka K, Yoshida T, Takakura Y, Umekawa T, Kogure A, Toda H, et al. Association study of g1704t and g82s polymorphisms of rage gene for microalbuminuria in japanese type 2 diabetic patients. Metabolism. 2005;54:488-491

106. Yoshioka K, Yoshida T, Takakura Y, Umekawa T, Kogure A, Toda H, et al. Relation between polymorphisms g1704t and g82s of rage gene and diabetic retinopathy in japanese type 2 diabetic patients. Intern Med. 2005; 44:417-421

107. Kankova K, Beranek M, Hajek D, Vlkova E. Polymorphisms 1704g/t, 2184a/g, and 2245g/a in the rage gene are not associated with diabetic retinopathy in niddm: Pilot study. Retina. 2002;22:119-121

108. Kumaramanickavel G, Ramprasad VL, Sripriya S, Upadyay NK, Paul PG, Sharma T. Association of gly82ser polymorphism in the rage gene with diabetic retinopathy in type ii diabetic asian indian patients. J Diabetes Complications. 2002;16:391-394

109. Liu L, Xiang K. Rage gly82ser polymorphism in diabetic microangiopathy. Diabetes Care. 1999;22:646

110. Matsunaga-Irie S, Maruyama T, Yamamoto Y, Motohashi Y, Hirose H, Shimada A, et al. Relation between development of nephropathy and the p22phox c242t and receptor for advanced glycation end product g1704t gene polymorphisms in type 2 diabetic patients. Diabetes Care. 2004;27:303-307

111. Li J, Schmidt AM. Characterization and functional analysis of the promoter of rage, the receptor for advanced glycation end products. J Biol Chem. 1997;272:16498-16506

112. Dworacka M, Winiarska H, Szymanska M, Szczawinska K, Wierusz-Wysocka B. Serum n-epsilon(carboxymethyl)lysine is elevated in nondiabetic coronary heart disease patients. J Basic Clin Physiol Pharmacol. 2002;13:201-213

113. Sugiyama S, Miyata T, Ueda Y, Tanaka H, Maeda K, Kawashima S, et al. Plasma levels of pentosidine in diabetic patients: An advanced glycation end product. J Am Soc Nephrol. 1998;9:1681-1688

114. Yoshida N, Okumura K, Aso Y. High serum pentosidine concentrations are associated with increased arterial stiffness and thickness in patients with type 2 diabetes. Metabolism. 2005;54:345-350

115. Takahashi R, Imamura A, Yoshikane M, Suzuki M, Murakami R, Cheng XW, et al. High serum concentrations of pentosidine, an advanced glycation end product, are associated with low normal value of ankle-brachial index in apparently healthy men. Metabolism. 2011;60:649-654

116. Semba RD, Ferrucci L, Sun K, Beck J, Dalal M, Varadhan R, et al. Advanced glycation end products and their circulating receptors predict cardiovascular disease mortality in older community-dwelling women. Aging Clin Exp Res. 2009;21:182-190

117. Semba RD, Bandinelli S, Sun K, Guralnik JM, Ferrucci L. Plasma carboxymethyl-lysine, an advanced glycation end product, and all-cause and cardiovascular disease mortality in older community-dwelling adults. J Am Geriatr Soc. 2009;57:1874-1880

118. Kilhovd BK, Berg TJ, Birkeland KI, Thorsby P, Hanssen KF. Serum levels of advanced glycation end products are increased in patients with type 2 diabetes and coronary heart disease. Diabetes Care. 1999;22:15431548

119. Baumann M, Richart T, Sollinger D, Pelisek J, Roos M, Kouznetsova T, et al. Association between carotid diameter and the advanced glycation end product n-epsilon-carboxymethyllysine (cml). Cardiovasc Diabetol. 2009;8:45

120. Busch M, Franke S, Wolf G, Brandstadt A, Ott U, Gerth J, et al. The advanced glycation end product $\mathrm{n}$ (epsilon)-carboxymethyllysine is not a predictor of cardiovascular events and renal outcomes in patients with type 2 diabetic kidney disease and hypertension. Am J Kidney Dis. 2006;48:571-579

121. Lund SS, Tarnow L, Stehouwer CD, Schalkwijk CG, Frandsen M, Smidt UM, et al. Targeting hyperglycaemia with either metformin or repaglinide in non-obese patients with type 2 diabetes: Results from a randomized crossover trial. Diabetes Obes Metab. 2007;9:394-407

122. Henry RM, Kostense PJ, Spijkerman AM, Dekker JM, Nijpels G, Heine RJ, et al. Arterial stiffness increases with deteriorating glucose tolerance status: The hoorn study. Circulation. 2003;107:2089-2095 
123. Thewissen MM, Damoiseaux JG, Duijvestijn AM, van Greevenbroek MM, van der Kallen CJ, Feskens EJ, et al. Abdominal fat mass is associated with adaptive immune activation: The codam study. Obesity (Silver Spring). 2011

124. Jacobs M, van Greevenbroek MM, van der Kallen CJ, Ferreira I, Blaak EE, Feskens EJ, et al. Low-grade inflammation can partly explain the association between the metabolic syndrome and either coronary artery disease or severity of peripheral arterial disease: The codam study. Eur J Clin Invest. 2009;39:437-444

125. Spijkerman AM, Adriaanse MC, Dekker JM, Nijpels G, Stehouwer CD, Bouter LM, et al. Diabetic patients detected by population-based stepwise screening already have a diabetic cardiovascular risk profile. Diabetes Care. 2002;25:1784-1789

126. Nin JW, Jorsal A, Ferreira I, Schalkwijk CG, Prins MH, Parving HH, et al. Higher plasma levels of advanced glycation end products are associated with incident cardiovascular disease and all-cause mortality in type 1 diabetes: A 12-year follow-up study. Diabetes Care. 2011;34:442-447

127. Scheijen JL, van de Waarenburg MP, Stehouwer CD, Schalkwijk CG. Measurement of pentosidine in human plasma protein by a single-column high-performance liquid chromatography method with fluorescence detection. J Chromatogr B Analyt Technol Biomed Life Sci. 2009;877:610-614

128. Kilhovd BK, Juutilainen A, Lehto S, Ronnemaa T, Torjesen PA, Hanssen KF, et al. Increased serum levels of methylglyoxal-derived hydroimidazolone-age are associated with increased cardiovascular disease mortality in nondiabetic women. Atherosclerosis. 2009;205:590-594

129. Brownlee M. Advanced protein glycosylation in diabetes and aging. Annu Rev Med. 1995;46:223-234

130. Giardino I, Edelstein D, Brownlee M. Nonenzymatic glycosylation in vitro and in bovine endothelial cells alters basic fibroblast growth factor activity. A model for intracellular glycosylation in diabetes. J Clin Invest. 1994;94:110-117

131. Daimon M, Ono Y, Saito T, Yamaguchi H, Hirata A, Ohnuma H, et al. Increased serum levels of pentosidine, but not carboxymethyl lysine, in type 2 diabetes without obvious diabetic nephropathy. Diabetes Care. 1999;22:877-878

132. Endo M, Yanagisawa K, Tsuchida K, Okamoto $T$, Matsushita $T$, Higuchi $M$, et al. Increased levels of vascular endothelial growth factor and advanced glycation end products in aqueous humor of patients with diabetic retinopathy. Horm Metab Res. 2001;33:317-322

133. Wagner Z, Wittmann I, Mazak I, Schinzel R, Heidland A, Kientsch-Engel R, et al. N(epsilon)(carboxymethyl)lysine levels in patients with type 2 diabetes: Role of renal function. Am J Kidney Dis. 2001;38:785-791

134. Semba RD, Beck J, Sun K, Egan JM, Carlson OD, Varadhan R, et al. Relationship of a dominant advanced glycation end product, serum carboxymethyl-lysine, and abnormal glucose metabolism in adults: The baltimore longitudinal study of aging. J Nutr Health Aging. 2010;14:507-513

135. Baidoshvili A, Krijnen PA, Kupreishvili K, Ciurana C, Bleeker W, Nijmeijer R, et al. N(epsilon)(carboxymethyl)lysine depositions in intramyocardial blood vessels in human and rat acute myocardial infarction: A predictor or reflection of infarction? Arterioscler Thromb Vasc Biol. 2006;26:2497-2503

136. Schleicher ED, Wagner E, Nerlich AG. Increased accumulation of the glycoxidation product n(epsilon)(carboxymethyl)lysine in human tissues in diabetes and aging. J Clin Invest. 1997;99:457-468

137. Nakamura $Y$, Horii $Y$, Nishino T, Shiiki H, Sakaguchi $Y$, Kagoshima T, et al. Immunohistochemical localization of advanced glycosylation end products in coronary atheroma and cardiac tissue in diabetes mellitus. Am J Pathol. 1993;143:1649-1656

138. Kume S, Takeya M, Mori T, Araki N, Suzuki H, Horiuchi S, et al. Immunohistochemical and ultrastructural detection of advanced glycation end products in atherosclerotic lesions of human aorta with a novel specific monoclonal antibody. Am J Pathol. 1995;147:654-667

139. Schalkwijk CG, Baidoshvili A, Stehouwer CD, van Hinsbergh VW, Niessen HW. Increased accumulation of the glycoxidation product nepsilon-(carboxymethyl)lysine in hearts of diabetic patients: Generation and characterisation of a monoclonal anti-cml antibody. Biochim Biophys Acta. 2004;1636:82-89

140. Baynes JW, Thorpe SR. Role of oxidative stress in diabetic complications: A new perspective on an old paradigm. Diabetes. 1999;48:1-9

141. Monnier VM, Vishwanath V, Frank KE, Elmets CA, Dauchot P, Kohn RR. Relation between complications of type i diabetes mellitus and collagen-linked fluorescence. N Engl J Med. 1986;314:403-408

142. Monnier VM, Bautista O, Kenny D, Sell DR, Fogarty J, Dahms W, et al. Skin collagen glycation, glycoxidation, and crosslinking are lower in subjects with long-term intensive versus conventional therapy of type 1 diabetes: Relevance of glycated collagen products versus hba1c as markers of diabetic complications. Dcct skin collagen ancillary study group. Diabetes control and complications trial. Diabetes. 1999;48:870-880 
143. Meerwaldt R, Hartog JW, Graaff R, Huisman RJ, Links TP, den Hollander NC, et al. Skin autofluorescence, a measure of cumulative metabolic stress and advanced glycation end products, predicts mortality in hemodialysis patients. J Am Soc Nephrol. 2005;16:3687-3693

144. Meerwaldt R, Graaff R, Oomen PH, Links TP, Jager JJ, Alderson NL, et al. Simple non-invasive assessment of advanced glycation endproduct accumulation. Diabetologia. 2004;47:1324-1330

145. Bos DC, de Ranitz-Greven WL, de Valk HW. Advanced glycation end products, measured as skin autofluorescence and diabetes complications: A systematic review. Diabetes Technol Ther. 2011;13:773-779

146. Brands PJ, Hoeks AP, Willigers J, Willekes C, Reneman RS. An integrated system for the non-invasive assessment of vessel wall and hemodynamic properties of large arteries by means of ultrasound. Eur $J$ Ultrasound. 1999;9:257-266

147. Peters SA, Grobbee DE, Bots ML. Carotid intima-media thickness: A suitable alternative for cardiovascular risk as outcome? Eur J Cardiovasc Prev Rehabil. 2011;18:167-174

148. Criqui MH, Fronek A, Klauber MR, Barrett-Connor E, Gabriel S. The sensitivity, specificity, and predictive value of traditional clinical evaluation of peripheral arterial disease: Results from noninvasive testing in a defined population. Circulation. 1985;71:516-522

149. Arain FA, Ye Z, Bailey KR, Chen Q, Liu G, Leibson CL, et al. Survival in patients with poorly compressible leg arteries. J Am Coll Cardiol. 2012;59:400-407

150. Abedin M, Tintut Y, Demer LL. Vascular calcification: Mechanisms and clinical ramifications. Arterioscler Thromb Vasc Biol. 2004;24:1161-1170

151. Yang EY, Chambless L, Sharrett AR, Virani SS, Liu X, Tang Z, et al. Carotid arterial wall characteristics are associated with incident ischemic stroke but not coronary heart disease in the atherosclerosis risk in communities (aric) study. Stroke. 2012;43:103-108

152. Dijk JM, Algra A, van der Graaf Y, Grobbee DE, Bots ML. Carotid stiffness and the risk of new vascular events in patients with manifest cardiovascular disease. The smart study. Eur Heart J. 2005;26:1213-1220

153. Barenbrock M, Kosch M, Joster E, Kisters K, Rahn KH, Hausberg M. Reduced arterial distensibility is a predictor of cardiovascular disease in patients after renal transplantation. J Hypertens. 2002;20:79-84

154. Blacher J, Pannier B, Guerin AP, Marchais SJ, Safar ME, London GM. Carotid arterial stiffness as a predictor of cardiovascular and all-cause mortality in end-stage renal disease. Hypertension. 1998;32:570-574

155. World Health Organization. Diabetes: Fact sheet no. 312. 2011;Accessed February 2012

156. Danaei G, Finucane MM, Lu Y, Singh GM, Cowan MJ, Paciorek CJ, et al. National, regional, and global trends in fasting plasma glucose and diabetes prevalence since 1980: Systematic analysis of health examination surveys and epidemiological studies with 370 country-years and 2.7 million participants. Lancet. 2011;378:31-40

157. International Diabetes Federation. Idf diabetes atlas: The global burden, 5th edition [online]. 2011;Accessed February 2012

158. The Emerging Risk Factors Collaboration, Sarwar N, Gao P, Seshasai SR, Gobin R, Kaptoge S, et al. Diabetes mellitus, fasting blood glucose concentration, and risk of vascular disease: A collaborative meta-analysis of 102 prospective studies. Lancet. 2010;375:2215-2222

159. The Emerging Risk Factors Collaboration, Seshasai SR, Kaptoge S, Thompson A, Di Angelantonio E, Gao P, et al. Diabetes mellitus, fasting glucose, and risk of cause-specific death. N Engl J Med. 2011;364:829-841

160. Wilson PW, D'Agostino RB, Levy D, Belanger AM, Silbershatz H, Kannel WB. Prediction of coronary heart disease using risk factor categories. Circulation. 1998;97:1837-1847

161. Conroy RM, Pyorala K, Fitzgerald AP, Sans S, Menotti A, De Backer G, et al. Estimation of ten-year risk of fatal cardiovascular disease in europe: The score project. Eur Heart J. 2003;24:987-1003

162. del Sol Al, Moons KG, Hollander M, Hofman A, Koudstaal PJ, Grobbee DE, et al. Is carotid intima-media thickness useful in cardiovascular disease risk assessment? The rotterdam study. Stroke. 2001;32:1532-1538

163. Lorenz MW, Schaefer C, Steinmetz H, Sitzer M. Is carotid intima media thickness useful for individual prediction of cardiovascular risk? Ten-year results from the carotid atherosclerosis progression study (caps). Eur Heart J. 2010;31:2041-2048

164. Polak JF, Pencina MJ, Pencina KM, O'Donnell CJ, Wolf PA, D'Agostino RB, Sr. Carotid-wall intima-media thickness and cardiovascular events. N Engl J Med. 2011;365:213-221

165. Elias-Smale SE, Kavousi M, Verwoert GC, Koller MT, Steyerberg EW, Mattace-Raso FU, et al. Common carotid intima-media thickness in cardiovascular risk stratification of older people: The rotterdam study. Eur J Cardiovasc Prev Rehabil. 2011 
166. Nambi V, Chambless L, Folsom AR, He M, Hu Y, Mosley T, et al. Carotid intima-media thickness and presence or absence of plaque improves prediction of coronary heart disease risk: The aric (atherosclerosis risk in communities) study. J Am Coll Cardiol. 2010;55:1600-1607 
SAMEMNATTING

M M AUVTTHS 


\section{SAMENVATTING}

Diabetes is een veelvoorkomende stofwisselingsziekte die gekenmerkt wordt door hyperglykemie (verhoogde bloedsuikerspiegels) als gevolg van gebreken in de productie en/of de werking van insuline. Op dit moment lijden wereldwijd ongeveer 346 miljoen mensen aan diabetes en naar verwachting zal dit aantal toenemen naar 552 miljoen in 2030. In Nederland waren er, in 2007, 740.000 mensen met diabetes en naar verwachting zal dit aantal ook toenemen, naar 1.3 miljoen in 2025 . Ongeveer $90 \%$ van alle mensen met diabetes heeft diabetes type 2. Hoewel diabetes type 2 vooral het gevolg is van verminderende insulinegevoeligheid, dat wil zeggen, weefsels worden minder gevoelig voor de metabole effecten van insuline, is de productie van insuline, door de bètacellen van de alvleesklier, ook defect bij deze patiënten en onvoldoende om te compenseren voor de insulineresistentie. Overgewicht en lichamelijke inactiviteit, die voornamelijk de gevoeligheid voor insuline beïnvloeden, worden beschouwd als de belangrijkste risicofactoren voor het ontstaan van diabetes type 2 . De meest voorkomende gevolgen, of complicaties, van diabetes die zorgen voor een verhoogd risico op ziekte en sterfte bij mensen met diabetes zijn microvasculaire complicaties zoals retinopathie (oogaandoening), nefropathie (nieraandoening) en neuropathie (zenuwschade) en macrovasculaire complicaties zoals atherosclerose (aderverkalking). Volwassen mensen met diabetes ervaren bijvoorbeeld een tweevoudig verhoogd risico op hart- en vaatziekten en aan hart- en vaatziekten gerelateerde sterfte ten opzichte van mensen zonder diabetes en onderzoek naar potentiele mechanismen ter verklaring van dit verhoogde risico is daarmee van belang.

De mechanismen die ten grondslag liggen aan de ontwikkeling van micro- en macrovasculaire complicaties (door hyperglykemie) bij mensen met diabetes zijn niet geheel duidelijk, maar de verhoogde versuikering van eiwitten en de daarmee gepaard gaande productie van vergevorderde glyceringsproducten [EN: advanced glycation endproducts (AGE's)] beslaat een mogelijk mechanisme. In dit proefschrift hebben wij dit betiteld als de glyceringsroute (EN: the glycation pathway). Tot dusver is het bewijs voor de rol van de glyceringsroute in (aan diabetes gerelateerde) hart- en vaatziekten voornamelijk afkomstig van (dier)experimentele studies. Het doel van dit proefschrift was dan ook om, in een aantal epidemiologische studies, mogelijke interventies in de glyceringsroute te evalueren (deel 1) en de rol van de glyceringsroute in het ontstaan van hart- en vaatziekten (deel 2) bij mensen met of zonder diabetes type 2 te onderzoeken.

Hoofdstuk 1 introduceert het onderzoek in dit proefschrift en geeft achtergrondinformatie over diabetes (in het bijzonder diabetes type 2), over aan diabetes 
gerelateerde micro- en macrovasculaire complicaties en de mogelijke rol van de glyceringsroute (versuikering van eiwitten) hierin.

\section{DEEL 1: INTERVENTIES IN DE GLYCERINGSROUTE}

Hoofdstuk 2 geeft een samenvatting van de huidige literatuur met betrekking tot mogelijke AGE's-remmende effecten van verschillende therapieën, hetzij specifiek ontwikkeld voor remming van AGE's of ontwikkeld voor interventies los van op de glyceringsroute gebaseerde theorieën. Hoewel (dier)experimentele studies met betrekking tot de specifieke AGE's-remmer aminoguanidine en de AGE's-crosslinkbreaker alagebrium duidelijk gunstige effecten hebben laten zien, lijkt het erop dat de veiligheid en/of werkzaamheid in humane/klinische studies met deze interventies verontrustend zijn. Met betrekking tot andere therapieën ontwikkeld voor doeleinden die geen verband houden met versuikering van eiwitten, maar die AGE's-remmende effecten hebben laten zien in voorgaande in vitro en/of dierexperimentele studies, zoals de B-vitamines pyridoxamine en thiamine, het bloedsuikerverlagende medicijn metformine en de bloeddrukverlagende angiotensine receptor blokkers (ARB's), bleek het huidige bewijs vrij beperkt. Dit lijkt voornamelijk het gevolg van een grote heterogeniteit in de opzet van de verschillende tot op heden gepubliceerde studies en/of de hierin gebruikte meettechnieken die vaak suboptimaal zijn geweest. Meer grote, specifieke en goed opgezette studies zijn nodig om hun potentiële AGE's-remmende effecten volledig op te helderen.

Gezien de overeenkomsten in structuur tussen het bloedsuikerverlagende medicijn metformine en de specifieke AGE's-remmer aminoguanidine, is er in de literatuur voorgesteld dat metformine kan zorgen voor detoxificatie van voorlopers van AGE's [zoals de dicarbonylverbindingen methylglyoxal en 3-deoxyglucosone (3DG)] op een vergelijkbare manier als aminoguanidine. Dit zou hiermee de gunstige effecten van metformine op hart- en vaatziekten bij diabetes type 2 gedeeltelijk kunnen verklaren. Eerdere (observationele) studies bij mensen met diabetes type 2 hebben inderdaad positieve 'effecten' aangetoond van metformine op een reeks van markers van de glyceringsroute. Hoewel het onderzoek in Hoofdstuk 3 eveneens een daling [-19,3\% (95\% BI: $-23,5$ tot $-14,8)$ ] in bloedwaarden van de dicarbonylverbinding 3DG liet zien na behandeling met metformine bij niet-obese patiënten met diabetes type 2, vonden we, opmerkelijk genoeg, een vergelijkbare daling $[-20,8 \%(-24,9$ tot $-16,3)]$ in 3DG niveaus na behandeling met een ander bloedsuikerverlagend medicijn, repaglinide. De verschillende werkingsmechanismen van de bloedsuikerverlagende effecten van metformine en repaglinide suggereren dus dat de verbetering van de glucosehuishouding, eerder dan een specifiek effect van metformine op de detoxificatie van dicarbonylverbindingen, resulteert 
in een afname van 3DG. Desalniettemin vonden we dat de zowel door metformine als door repaglinide geïnduceerde vermindering van 3DG samenhing met een vermindering van markers van disfunctie van het endotheel (binnenbekleding van de bloedvaten) en als zodanig dus een metabole route kan vormen waarlangs beide behandelingen een gunstig effect hebben op het risico op hart- en vaatziekten in patiënten met diabetes type 2 .

De remmende werking van ARB's op AGE's en op de gevolgen van AGE's, zoals dit eerder is aangetoond in verschillende in vitro en dierexperimentele studies, heeft tot de hypothese geleid dat deze AGE's-remmende effecten, wellicht voor een deel, de gunstige effecten van ARB's op het risico op hart- en vaatziekten bij patiënten met diabetes type 2 kunnen verklaren. Echter, hoewel een aantal eerder gepubliceerde kleine klinische studies, veelal zonder controlegroep, inderdaad dalingen hebben laten zien in AGE's in het bloedplasma of urine na behandeling met ARB's, vonden wij in de grote gerandomiseerde gecontroleerde klinische studie [EN: randomised controlled trial (RCT)] in Hoofdstuk 4 geen effecten op bloedplasmaniveaus van de $A G E^{\prime} s N^{\varepsilon}$-(carboxymethyl)lysine (CML) en $N^{\varepsilon}$ (carboxyethyl)lysine (CEL) van twee jaar behandeling met de ARB irbesartan bij mensen met diabetes type 2 . Onze bevindingen bevestigen de enige twee andere grote RCT's die dit tot nu toe hebben onderzocht en geen AGE's-remmende effecten vonden van behandeling met irbesartan of eprosartan (een andere ARB).

\section{DEEL 2: DE GLYCERINGSROUTE EN HART- EN VAATZIEKTEN}

In Hoofdstuk 5 worden referentie-intervallen gepresenteerd voor metingen van de dikte van de halsslagaderwand [EN: carotid intima-media thickness (CIMT)], een meting die, in zowel onderzoek als steeds meer ook in de klinische praktijk, gebruikt wordt als marker van atherosclerose maar waarvan zulk soort referentie-intervallen tot op heden ontbraken. Deze referentie-intervallen zijn vastgesteld op basis van een steekproef ( $n=24,871$ ) van samengevoegde CIMT data van gezonde proefpersonen eerder gemeten in 24 verschillende studiecentra wereldwijd. Verder hebben we in dit hoofdstuk relaties onderzocht tussen bekende risicofactoren voor hart- en vaatziekten enerzijds en het afwijken van de voorspelde 'normale' CIMT waarde voor een bepaalde leeftijd en geslacht anderzijds. Dit maakt een accurate vergelijking mogelijk van CIMT waarden binnen en tussen groepen met verschillende risicoprofielen, in het bijzonder met betrekking tot het ontwikkelen van hart- en vaatziekten, en helpt daarmee de interpretatie van dergelijke metingen verkregen zowel in onderzoek als in de klinische praktijk.

In Hoofdstuk 6 hadden we de hypothese dat genetische variatie in het gen dat codeert voor glyoxalase 1 (GLO1; het snelheidsbepalende enzym in de detoxificatie van de voorlopers van AGE's methylglyoxal en glyoxal) de expressie en/of de activiteit van GLO1 kan beïnvloeden en als zodanig het genetisch bepaalde risico op hart- en vaatziekten kan 
beïnvloeden. Hiertoe hadden we negen veelvoorkomende enkel-nucleotide polymorfismen [EN: single nucleotide polymorphisms (SNP's); variaties in het DNA van een enkele nucleotide lang] bepaald bij deelnemers van twee Nederlandse cohorten van mensen met een 'normaal' glucosemetabolisme, een gestoord glucose metabolisme of diabetes type 2 (de Hoorn en CODAM studies). Door het bepalen van deze negen SNP's konden we de totale veelvoorkomende genetische variatie in GLO1 onderzoeken. Echter, we vonden geen bewijs voor (consistente) relaties tussen de negen GLO1 SNP's enerzijds en de prevalentie van hypertensie (hoge bloeddruk), markers van atherosclerose (waaronder CIMT), stijfheid van de bloedvaten of nierfunctie anderzijds.

Eerdere studies hebben aangetoond dat genetische variatie in het gen dat codeert voor de receptor voor AGE's [EN: receptor for advanced glycation endproducts (RAGE)] de expressie en activiteit van RAGE en/of de affiniteit voor het binden van liganden aan deze receptor kan beïnvloeden. Ook RAGE SNP's zouden als zodanig het genetisch bepaalde risico op hart- en vaatziekten kunnen beïnvloeden. Tot dusver heeft onderzoek naar de relatie tussen RAGE SNP's enerzijds en hart- en vaatziekten anderzijds tegenstrijdige resultaten laten zien die mogelijk veroorzaakt zijn door verschillen tussen proefpersonen in onder andere het glucosemetabolisme (dat wil zeggen of zij diabetes type 1, diabetes type 2 of een 'normaal' glucosemetabolisme hebben). Onze bevindingen in Hoofdstuk 7 ondersteunen deze hypothese van een invloed van het glucosemetabolisme op de relatie tussen RAGE SNP's enerzijds en hart- en vaatziekten anderzijds. Het onderzoek in dit hoofdstuk laat een beschermende rol op het gebied van bloeddruk en stijfheid van de bloedvaten zien van het AA genotype van RAGE SNP -374T/A bij mensen met een 'normaal' glucosemetabolisme, maar een schadelijk effect bij personen met een gestoord glucosemetabolisme of diabetes type 2 .

Om de rol van de glyceringsroute in hart- en vaatziekten te onderzoeken, hebben we tenslotte bloedplasmaniveaus gemeten van zowel aan eiwit gebonden (CML, CEL en pentosidine) als vrij circulerende [CML, CEL en 5-hydro-5-methylimidazolone (MG-H1)] AGE's, wederom bij deelnemers van de Hoorn en CODAM studies. In Hoofdstuk 8 vonden we echter geen (onafhankelijke) relaties tussen bloedplasmaniveaus van de eiwitgebonden en vrije AGE's enerzijds en prevalente hart- en vaatziekten, of markers van atherosclerose (waaronder CIMT) anderzijds.

Kort gezegd hebben we in het onderzoek dat beschreven staat in dit proefschrift, geen bewijs gevonden voor (specifieke) AGE's-remmende effecten van het bloedsuikerverlagende medicijn metformine en ook niet van het bloeddrukverlagende medicijn irbesartan. Daarnaast vonden we geen (negatieve) verbanden van circulerende AGE's (gemeten in het bloedplasma) met HVZ of markers daarvan. Er moet echter worden benadrukt dat het op dit moment onzeker is of verhoogde bloedplasmaniveaus van AGE's de last van versuikering van eiwitten in de weefsels van mensen met of zonder diabetes 
type 2 adequaat weergeven. Het onderzoek beschreven in dit proefschrift ondersteunt dus niet noodzakelijkerwijs het ontbreken van een rol voor versuikering van eiwitten in aan diabetes (type 2) gerelateerde hart- en vaatziekten bij de mens, maar toont het belang aan van identificatie van (andere) valide markers die de last van versuikering van eiwitten in de weefsels wellicht beter weerspiegelen. Een dergelijke marker kan dan, naast zijn waarde in epidemiologische studies, bijvoorbeeld zoals beschreven in dit proefschrift, een mogelijk bruikbaar instrument zijn in de identificatie van individuen met een verhoogd risico voor het ontwikkelen van diabetes en/of hart- en vaatziekten. 


\section{DAKKSYARRD}




\section{DANKWOORD}

Het onderzoek beschreven in dit proefschrift en het schrijven en afronden van het boekje zelf was natuurlijk alleen mogelijk met de hulp van velen. Ik wil dan ook graag een poging doen iedereen te bedanken die een belangrijke bijdrage heeft geleverd aan het tot stand komen van mijn proefschrift.

Voor het onderzoek, dat heeft geresulteerd in dit proefschrift, heb ik gebruik mogen maken van data van verschillende epidemiologische (cohort)studies. Allereerst zou ik dan ook graag alle 'principal investigators' en onderzoeks- en andere medewerkers willen bedanken die deze studies tot een succes hebben gemaakt. Veel onderzoekers, zoals ikzelf, hebben daardoor de mogelijkheid nu en in de toekomst interessante wetenschappelijke vragen te onderzoeken.

Prof. dr. C.G. Schalkwijk, prof. dr. C.D.A. Stehouwer en dr. I. Ferreira, Casper, Coen en Isabel, wat een promotieteam heb ik achter me gehad de afgelopen (iets meer dan) vier jaar! Jullie bewonderenswaardige en vooral ook uiteenlopende expertise en buitengewone toewijding hebben ervoor gezorgd dat elk hoofdstuk tot in perfectie werd uitgewerkt. Elk gezamenlijk overleg motiveerde mij weer om er iets moois (mooiers) van te maken. Zelfs gedurende mijn periode in Parijs verliep de communicatie (ware het via email) uitzonderlijk goed en gingen we gestaag door met schrijven, 'rebuttelen' en publiceren. Vooral ook toen besefte ik in welk bijzonder team ik terecht was gekomen, werkelijk fantastisch. Bovendien ben ik jullie zeer dankbaar voor alle kansen die ik gekregen heb, zoals het volgen van, naar mijn beleving oneindig veel, cursussen en het bezoeken van congressen, het buitenlandbezoek gedurende mijn promotietijd en de vele (inter)nationale contacten die ik via jullie heb opgedaan. Dit alles heeft niet alleen bijgedragen aan mijn wetenschappelijke ontwikkeling, maar zorgde ook voor (nog meer) enthousiasme voor de wetenschap, ook zeker niet onbelangrijk!

Casper, jouw ongelofelijk open en prettige manier van werken en doen en laten [niet alleen je deur die altijd open staat wanneer iemand een vraag of probleem heeft, maar ook je gastvrijheid (en die van Hermien) bij lab-uitjes en kerstdiners] zijn naar mijn mening de basis van de fantastische werksfeer die heerst op 'onze gang'. Daarnaast weet je door jouw kennis en enthousiasme over eiwitglycering (en natuurlijk zoveel meer) en wetenschap in het algemeen iedereen om je heen te motiveren, kansen te benutten en weet je vaak door kleine aanpassingen een manuscript of presentatie naar een hoger niveau te brengen. Je ogenschijnlijk onuitputtelijke motivatie om onze negatieve resultaten toch in 'hoog impact-journals' te publiceren, al moesten hiervoor de nodige 
zeer overtuigende brieven geschreven worden, is een mooi voorbeeld van je toewijding en heeft mij ondanks deze kleine tegenslagen altijd gemotiveerd gehouden.

Coen, ik vraag me nog altijd af hoe jij het overzicht behoudt ondanks je ongelofelijk drukke agenda (wat een understatement), verscheidenheid aan taken en grote groep promovendi met toch behoorlijk uiteenlopende onderzoeken die je begeleidt. Juist dit overzicht maakt jou naar mijn mening een ideale promotor. Je weet altijd een oplossing, geeft de kern in een paar woorden perfect weer, maar haalt ook (kleine en iets grotere) missers er altijd uit. Dat je dit ook nog iedere keer weer binnen ongelofelijk korte tijd weet te doen, is werkelijk uitzonderlijk en maakt werken onder jouw supervisie zeer prettig.

Isabel, wat moet ik meer zeggen dan hoe fantastisch ik die maandagmiddagen altijd heb gevonden waarop we niet alleen tot in de diepte de analyses bespraken en vaak samen uitvoerden, jij me wegwijs maakte in het effectief, eenduidig en aantrekkelijk wetenschappelijk schrijven, maar vooral ook altijd klaarstond om andere (privé)verhalen aan te horen (of me even lekker weg te sturen naar een prettig Portugees verblijf ;)), of zelf op je eigen manier mij te leren over de wetenschap, 'het wetenschappelijke wereldje' en zoveel meer. Jouw grondige epidemiologische kennis en vaardigheden en je ongelofelijke drive om (nieuwe) technieken tot op de bodem uit te zoeken hebben ervoor gezorgd dat elk hoofdstuk uiteindelijk uitgroeide tot een topstuk waar we allemaal trots op waren. Ik heb in zoveel opzichten ontzettend veel van je geleerd, bedankt Isabel!

Alle leden van de beoordelingscommissie, prof. dr. H.A.J. Struijker-Boudier, prof. dr. L.M.A. van Bortel, prof. dr. N.C. Schaper, prof. dr. Y.T. van der Schouw, dr. M.T. Schram en dr. A.J. Smit wil ik graag bedanken voor het lezen en beoordelen van mijn proefschrift.

Mijn paranimfen, kamergenootjes en fantastische collega's, Roel en Johanna, wat een topkamer is 5.312 toch altijd geweest! Zo fijn hoe wij al die jaren nagenoeg alles waar we tegenaan liepen als promovendi (eerst) met elkaar konden bespreken en vooral ook hebben besproken. Dezelfde frustraties, stressmomentjes, lastige perioden, maar vooral ook gelukmomentjes en vooruitgang doormaken, maakte onze band snel heel sterk. Ik heb veel geleerd van al onze (wetenschappelijke en minder wetenschappelijke) discussies en heb het vooral ook altijd heel prettig gevonden met jullie samen te werken, een kamer te delen, koffie te drinken, koekjes te eten, onze epidemiologie-, statistiek- maar ook computer-probleempjes uitvoerig te bespreken en uiteindelijk op te lossen, cursussen te volgen, congressen te bezoeken, en zoveel meer. Nu we alledrie (bijna) klaar zijn en jullie 5.312 en mij verlaten hebben om te specialiseren tot internist zijn onze contactmomentjes minder vanzelfsprekend en vinden nu voornamelijk plaats in leuke (Maastrichtse) restaurantjes en barretjes, maar ik moet eerlijk bekennen dat me dat inmiddels ook wel kan bevallen :). Misschien kunnen we in de toekomst de wetenschap samen nog eens oppakken! Ik vind het een eer dat jullie mijn paranimfen willen zijn. 
Mijn nieuwe kamergenootjes, Marcelle en Elisabeth. Het was best wel even wennen na vier jaar mijn Roel en Johanna :), maar al snel hadden we ons meidenkamertje een beetje anders ingericht en werd het meteen weer een gezellige boel. Jullie hebben me door de laatste loodjes heen geholpen, super bedankt! Ik vind het fijn dat we nog een tijdje op deze manier samen blijven werken.

Bas, ook al ben je nooit mijn kamergenoot geweest, we hebben voor mijn gevoel minstens net zo veel frustraties gedeeld en discussies gehad. Ik heb het altijd leuk gevonden hoe je onze kamer op kwam (en komt) stormen en in al je enthousiasme je (epidemiologische) probleem al uitgebreid op tafel had gelegd op het moment dat je besefte dat wij misschien wel zelf aan het werk waren. Als we dan samen aan de slag gingen, kwamen we er toch heel vaak uit en ik denk dat we hier allemaal veel aan gehad hebben. Zelf sta je altijd klaar voor een (goede) wetenschappelijke discussie (of ander fijn gesprek) als ikzelf of iemand anders ergens mee zit, altijd weer met evenveel enthousiasme. Je bent echt een fijne collega! Heel veel succes nog met jouw laatste loodjes.

Heel graag wil ik ook mijn overige collega's van het laboratorium MVM bedanken, wat een team! Echt zonder uitzondering is iedereen even gemotiveerd en enthousiast over zijn of haar werk, gaat voor de top, maar is daarnaast altijd in voor een praatje of gezelligheid op of buiten het werk. Ik heb dit altijd heel bijzonder gevonden en het geeft een fantastische werksfeer. Door jullie zijn die promotiejaren omgevlogen; de labuitjes, kerstfeestjes en borrels zijn altijd weer een feest en elk congres eigenlijk ook :). Ik vind het echt fijn dat ik nog een tijdje met jullie mag samenwerken. Marleen en Carla, bedankt voor al jullie hulp bij vragen over CODAM, SNPs en zoveel meer, fijn dat jullie deur altijd open staat. Alle postdocs en (oud)promovendi: Katrien en Olaf (ook bedankt voor al jullie hulp/tips als 'oudere-jaars-promovendi'), Kristiaan, Steven, Marjon, Matthijs, Amy, Rianne, Barry, Nordin, Hanneke, Nick, Dennis, Thomas (en alle andere Maastricht studie-postdocs en promovendi), Yvo, Teba en Umit. Ook al zijn de AIO-uitjes er een beetje uitgegaan in de loop van de tijd (hier moeten we toch eens iets aan gaan doen!), is het altijd gezellig, bedankt! Ook wil ik graag alle analisten van ons lab bedanken, Marjo, Petra, Vicky, Margee en Jean, jullie hebben allemaal wel op een bepaalde manier bijgedragen aan metingen voor de studies in mijn proefschrift, zonder jullie had ik simpelweg geen data, bedankt! Jean, jou wil ik graag nog extra bedanken voor het ontwerpen van de omslag en je hulp bij de lay-out en andere (computer)probleempjes door de jaren heen, echt ongelofelijk hoe je als vanzelfsprekend altijd voor iedereen klaarstaat.

Alle andere (CARIM)aio's, die ik heb leren kennen op cursussen en congressen in de afgelopen jaren, wil ik graag bedanken voor de leuke discussies en de gezelligheid. 
Ik wil ook graag alle coauteurs bedanken voor hun kritische blik op alle manuscripten en hun hulp in het verbeteren hiervan. In het bijzonder wil ik, van de Hoorn studie, Jacqueline Dekker, Giel Nijpels en Ronald Henry bedanken en, van de CODAM studie, Marleen van Greevenbroek, Carla van der Kallen, Ellen Blaak, Hugo ten Cate en Edith Feskens voor hun hulp in de totstandkoming van hoofdstukken 6, 7 en 8.

I would also like to thank all co-authors from the Steno Diabetes Center in Gentofte, Denmark who have not only provided me with the opportunity to use their data, but also improved the manuscripts (Chapter 3 and 4) with their comments and ideas. In particular, I would like to thank Frederik Persson, Søren Lund, Peter Rossing, Allan Vaag, Lise Tarnow, Peter Hovind and Hans-Henrik Parving.

For the research resulting in Chapter 5 of this thesis, I had the opportunity to spend eight months in the research group of prof. dr. S. Laurent and prof. dr. P. Boutouyrie within the Paris Cardiovascular Research Center of the George Pompidou Hospital in Paris, France. Pierre and Stéphane, thank you so much for everything. I had an exciting experience abroad, worked on an interesting project (for which we can hopefully continue our collaboration in the coming years) and met and collaborated with many European investigators in the field of vascular structure and function. I am very grateful for all these opportunities you gave me. In addition, I would like to thank all my Parisian colleagues (in particular Annie, Guillaume, Nicolas and Maureen, but of course also all others) for all their help and all the fun, not only in the hospital canteen ;) but also after work, I have had a wonderful time!

Gelukkig heb ik naast het werk ook nog een aantal hele fijne mensen om me heen die voor de nodige ontspanning zorgen en/of de mogelijkheid geven mijn hart te luchten als dat weer even nodig is :). In het bijzonder wil ik heel graag Jolijn, Debby en Inez bedanken voor alle gezelligheid en de goede gesprekken die we hebben tijdens onze etentjes (die houden we er nog heel lang in!!). Ik vind het zo fijn dat we alles kunnen bespreken en kan jullie nu echt beloven dat mijn 'gezeur' over dat boekje na 12 oktober eindelijk voorbij is... ;) Spannend dat jullie (Jolijn en Debby) nu ook begonnen zijn. Ik heb er bewondering voor dat jullie dat naast je werk gaan doen, het zal zwaar zijn, maar als het eenmaal zover is... FEEST!

Natuurlijk wil ik ook Tjandra (jij bent al zover), Nadine en Marianne (jullie zijn bijna aan de beurt), Kelly en Davy, Xavier, Kevin, Erik en Francis heel graag bedanken voor alle gezelligheid tijdens etentjes, feestjes en weekendjes weg, echt heerlijk.

Het is natuurlijk ook altijd even lekker ontspannen op de tennisbaan, vooral samen met Joep, Karel, Petra en Vera (maar natuurlijk ook alle anderen). Ook al heb ik jammer genoeg het laatste jaar een beetje moeten afhaken hier en daar (en daar ga ik weer aan 
werken :)), wil ik jullie graag bedanken voor alle gezelligheid en fijne gesprekken op en naast de tennisbaan.

Guy, bedankt voor alles, voor de fijne tijd die we samen hebben gehad. Jij wist me, met je altijd relaxte doen en laten altijd wel weer een beetje te kalmeren als ik gestresst raakte van mijn onderzoek of iets wat daarmee te maken had. Marc en Frieda, Kristel en Jef, Ann en Steven en Joren en Elise, en ook de rest van de 'Belgische gang', in het bijzonder Bert en Eveline, bedankt voor alle gezelligheid en interesse in mijn promotie (of vooral het feest eigenlijk;)).

Ten slotte, papa, mama en Aniek, jullie staan echt altijd onvoorwaardelijk voor mij klaar. Zeker in de laatste jaren waarin jullie al buitengewoon veel te verwerken hebben (gehad), is nooit iets te veel. Bedankt voor alles. 
CURRICULUM VITAE

RICULUM VITAE 


\section{CURRICULUM VITAE}

Lian Engelen was born on October $15^{\text {th }}, 1983$ in Kerkrade, the Netherlands. She graduated from secondary school (VWO, Eijkhagen College, Landgraaf, the Netherlands) in 2001. That same year she started her study in Health Sciences at Maastricht University. In 2005, a six-month internship at the department of Biomedical Sciences of the University of Wollongong, Australia resulted in obtaining her Master's degree in Human Movement Sciences. After graduation, she worked during one year as a junior researcher at the department of Plastic Surgery of Maastricht University Medical Centre ${ }^{+}$. Hereafter, in October 2007, she started her PhD research at the department of Internal Medicine within the CARIM School for Cardiovascular Disease of the same institution. Using several epidemiological studies, she studied the role of the glycation pathway in type 2 diabetes and cardiovascular disease. In addition, during this PhD period Lian spent eight months at the Paris Cardiovascular Research Centre (PARCC) of the George Pompidou Hospital in Paris, France. Here, she completed the Reference Values for carotid intima-media thickness project in collaboration with the Reference Values for Arterial Measurements Collaboration. At present, Lian is appointed as a postdoctoral researcher at the Top Institute Food and Nutrition (TIFN; theme Cardiovascular Health) within the department of Internal Medicine, Maastricht University Medical Centre ${ }^{+}$. 


\section{LIST OF PUBLLCATIOKS PUBLICATIDIS}




\section{LIST OF PUBLICATIONS}

Engelen L, Ferreira I, Brouwers O, Henry RMA, Dekker JM, Nijpels G, Heine RJ, van Greevenbroek MMJ, van der Kallen $\mathrm{CJH}$, Blaak EE, Feskens EJM, ten Cate $\mathrm{H}$, Stehouwer CDA, Schalkwijk CG. Polymorphisms in glyoxalase 1 gene are not associated with vascular complications: the Hoorn and CODAM studies. J Hypertens 2009; 27(7): 1399-1403.

Engelen L, Ferreira I, Gaens KHJ, Henry RMA, Dekker JM, Nijpels G, Heine RJ, 't Hart LM, van Greevenbroek MMJ, van der Kallen $\mathrm{CJH}$, Blaak EE, Feskens EJM, ten Cate H, Stehouwer CDA, Schalkwijk CG. The association between the -374T/A RAGE polymorphism and blood pressure and arterial stiffness is modified by glucose metabolism status. The Hoorn and CODAM studies. J Hypertens 2010; 28(2): 285-93.

Engelen L*, Lund SS*, Ferreira I, Tarnow L, Parving H-H, Gram J, Winther K, Pedersen O, Teerlink T, Barto R, Stehouwer CDA, Vaag AA, Schalkwijk CG. Improved glycemic control induced by both metformin and repaglinide is associated with a reduction in blood levels of 3-deoxyglucosone in non-obese patients with type 2 diabetes. Eur J Endocrinol 2011; 164(3): 371-379. *equal contribution

Engelen L, Persson F, Ferreira I, Rossing P, Hovind P, Teerlink T, Stehouwer CDA, Parving H$H$, Schalkwijk CG. Irbesartan treatment does not influence plasma levels of the advanced glycation end products $\mathrm{N}^{\varepsilon}$-(1-carboxymethyl)lysine and $\mathrm{N}^{\varepsilon}$ - (1-carboxyethyl)lysine in patients with type 2 diabetes and microalbuminuria. A randomized controlled trial. Nephrol Dial Transplant 2011; 26(11):3573-7.

Engelen L, Ferreira I, Stehouwer CDA, Boutouyrie P, Laurent S, on behalf of the Reference Values for Arterial Measurements Collaboration. Reference intervals for common carotid intima-media thickness measured with echotracking - relation with risk factors. Submitted for publication.

Hanssen NMJ*, Engelen L*, Ferreira I, Scheijen JLJM, Huijberts MS, van Greevenbroek MMJ, van der Kallen CJH, Dekker JM, Nijpels G, Stehouwer CDA, Schalkwijk CG. Plasma levels of advanced glycation endproducts are not independently associated with cardiovascular disease in individuals with or without type 2 diabetes: the Hoorn and CODAM Studies. Submitted for publication. *equal contribution 
Engelen L, Stehouwer CDA, Schalkwijk CG. Current interventions in the glycation pathway: evidence from clinical studies. Submitted for publication.

Caldwell JN, Engelen L, van der Henst C, Patterson M, Taylor NA. The interaction of body armour, low-intensity exercise and hot-humid conditions on physiological strain and cognitive function. Mil Med 2011; 176(5): 488-493. 